\title{
WestVirginiaUniversity
}

THE RESEARCH REPOSITORY @ WVU

Graduate Theses, Dissertations, and Problem Reports

2007

\section{DNA damage response activated by anti-cancer agent, irofulven}

Timothy D. Wiltshire

West Virginia University

Follow this and additional works at: https://researchrepository.wvu.edu/etd

\section{Recommended Citation}

Wiltshire, Timothy D., "DNA damage response activated by anti-cancer agent, irofulven" (2007). Graduate Theses, Dissertations, and Problem Reports. 2601.

https://researchrepository.wvu.edu/etd/2601

This Dissertation is protected by copyright and/or related rights. It has been brought to you by the The Research Repository @ WVU with permission from the rights-holder(s). You are free to use this Dissertation in any way that is permitted by the copyright and related rights legislation that applies to your use. For other uses you must obtain permission from the rights-holder(s) directly, unless additional rights are indicated by a Creative Commons license in the record and/ or on the work itself. This Dissertation has been accepted for inclusion in WVU Graduate Theses, Dissertations, and Problem Reports collection by an authorized administrator of The Research Repository @ WVU.

For more information, please contact researchrepository@mail.wvu.edu. 


\title{
DNA Damage Response Activated by Anti-cancer Agent, Irofulven
}

\author{
Timothy D. Wiltshire
}

Dissertation submitted to the School of Medicine at West Virginia University in partial fulfillment of the requirements for the degree of

\author{
Doctor of Philosophy \\ in \\ Cancer Cell Biology
}

\author{
Weixin Wang, Ph.D., Advisor \\ Steven Frisch, Ph.D., Chair \\ Yehenew Agazie, Ph.D. \\ Daniel Flynn, Ph.D. \\ Scott Weed, Ph.D. \\ Linda Vona-Davis, Ph.D.
}
Program in Cancer Cell Biology
West Virginia University
Morgantown, West Virginia
2007

Key words: DNA damage response, irofulven, chemosensitivity, BRCA1, DNA Repair, apoptosis, cell cycle 


\title{
Abstract
}

\section{DNA Damage Response Activated by Anti-cancer Agent, Irofulven.}

\author{
Timothy D. Wiltshire \\ Advisor: Weixin Wang, Ph.D. Assistant Professor, Department of Microbiology, \\ Immunology and Cell Biology.
}

The DNA damage response is a complex network of signals that coordinate to protect cells from accumulating mutations that lead to the development of cancer. Upon the introduction of DNA damage from either environmental or endogenous sources, the DNA damage response coordinates the control of cell cycle with DNA repair mechanisms to ensure genomic integrity within the cell. Mutations in proteins in this pathway lead to genomic instability and early onset of cancer. BRCA1 is a protein that plays a critical role in response to DNA damage caused by ionizing radiation and is responsible for approximately $50 \%$ of inherited breast and ovarian cancers. A key element in the response to DNA damage is the exact type of lesion produced. Irofulven represents a novel DNA damaging agent that may provide insight into specific signals involved. It also remains to be determined if tumors that have mutations in BRCA1 may be more or less sensitive to treatment with irofulven. The exact method by which irofulven kills cells also remains to be determined. Many chemotherapeutics are potent inducers of apoptosis and irofulven has been shown to activate elements of the apoptotic pathway. Previous work in our lab has shown the ability of irofulven to activate ATM and CHK2. Based on the fact that BRCA1 lies directly downstream in this pathway, we hypothesized that it plays a key role in the irofulven induced DNA damage response.

In our current study, we determined that BRCA1 plays a role in regulation of $\mathrm{S}$ and $\mathrm{G} 2 / \mathrm{M}$ cell cycle checkpoints after irofulven exposure. We also demonstrated that DNA repair via homologous recombination plays a role in response to DNA damage induced by irofulven and that cells deficient in such repair are more sensitive to irofulven. Lastly, we demonstrated that the activation of apoptosis by irofulven is regulated by caspases 2 and 9 , while caspase 8 seems to render cells resistant. Taken together, this study expanded our knowledge of signaling pathways activated by irofulven and provides a basis for targeted treatment in BRCA1 deficient breast and ovarian cancers. 


\section{Dedication}

This dissertation is dedicated to my wife, Kim Wiltshire, whose sacrifices at home and hard work to help support our family have given me the opportunity to achieve my

dreams. To my children, Luke and Ashlyn, for their smiling faces when I get home and their "help" in the lab. 


\section{Acknowledgements}

I am grateful to my mentor, Dr. Wang, for his instruction and guidance throughout my graduate training. I am thankful for his assistance and patience for the last

four years as we worked through my education together. My experience as West Virginia University has helped me develop as a scientist and will always be remembered.

I would also like to thank my committee members for their time, patience and advice. I have been privileged to have helpful and thoughtful committee members including Dr. Steven Frisch, Dr. Yehenew Agazie, Dr. Dan Flynn, Dr. Scott Weed and Dr. Lind Vona-Davis. I would especially like to thank Dr. Frisch and Dr. Weed for their input, advice and generous gifts of reagents for my experiments. 


\section{Table of Contents}

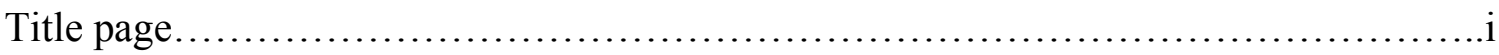

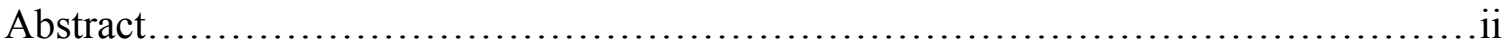

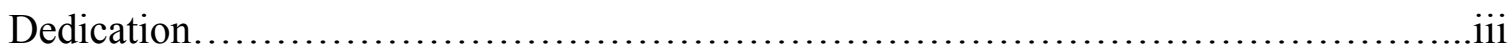

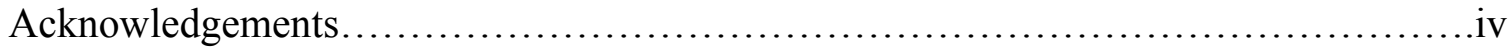

Table of contents.......................................................vi

List of abbreviations.......................................................... vii

\section{Chapter I}

Introduction and Review of Literature........................................

I. Cell Cycle Control.............................................1

II. DNA Repair..................................................5

III. DNA Damage Response Proteins.................................6

IV. Apoptosis...................................................15

V. Nonapoptotic Roles for Caspases.................................20

VI. Irofulven................................................21

VII. Summary and Significance....................................22

VIII. References....................................................24

\section{Chapter II}

BRCA1 Contributes to Cell Cycle Arrest and Chemoresistance in Response to the

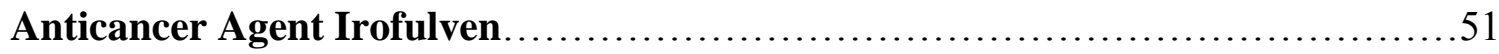

Abstract....................................................................... 52

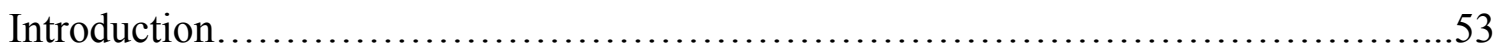


Materials and Methods

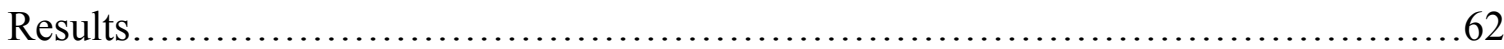

Discussion.............................................................69

Acknowledgements........................................................ 73

References............................................................. 74

Figure legends......................................................... 85

Figures.............................................................. 89

\section{Chapter III}

Caspase 2 Mediates Irofulven Induced Cell Death.......................... 103

Abstract................................................................. 104

Introduction............................................................. 105

Materials and Methods...................................................... 107

Results...................................................................... 110

Discussion.............................................................. 114

Aknowledgements.............................................................. 118

References............................................................ 119

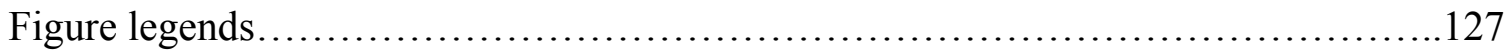

Figures................................................................ 130

\section{Chapter IV}

Role of Caspase 8 in Irofulven Induced Apoptosis.......................... 141

Abstract.................................................................... 142 
Introduction.

Materials and Methods.................................................... 147

Results............................................................. 150

Discussion............................................................ 153

References............................................................ 155

Figure legends....................................................... 165

Figures.............................................................. 167

\section{Chapter V}

General Discussion....................................................... 177

References............................................................ 186

\section{Appendix 1}

Fanconi anemia D2 protein confers chemoresistance in response to the anticancer agent, irofulven..................................................... 193

Appendix 2

ATM-dependent CHK2 Activation Induced by Anticancer Agent, Irofulven.....203

\section{Appendix 3}

Irofulven induces replication-dependent CHK2 activation related to p53 status...216

Curriculum Vitae. .226 


\section{List of Abbreviations}

Apaf-1 - Apoptosis activating factor 1

AT - Ataxia Telangiectasia

ATM - Ataxia Telangiectasia mutated

ATR - ATM and Rad3 related

BASC - BRCA1-associated genome surveillance complex

BER- Base excision repair

BRCA1 - Breast cancer associated 1

BRCA2 - Breast cancer associated 2

BRCT - BRCA1 C-terminal domain

Cdc25 - Cell cycle division dual specificity phosphatases

CDK - Cyclin-dependent kinase

DD - Death domain

DED - Death effector domain

DISC - Death-inducing signaling complex

DSB - Double strand break

ERK - extracellular signal-regulated kinase

FADD - Fas-associated death domain

FAK - Focal adhesion kinase

HR - Homologous recombination

ICL - Interstrand crosslinks

IR - Ionizing radiation 
JNK - c-Jun N-terminal kinase

NER- Nucleotide excision repair

NHEJ - Non-homologous end joining

PARP - Poly ADP ribose polymerase

PIDD - p53-induced protein with a death domain

TNFR - Tumor necrosis factor receptor

TNF $\alpha$ - Tumor necrosis factor $\alpha$

UV - Ultraviolet 


\section{Chapter I}

\section{Introduction and Review of the Literature}


The DNA damage response is a broad signaling network with the ability to sense damage to DNA and respond via multiple cellular mechanisms to ensure genomic integrity and prevent harmful mutations. Coordination of the DNA damage response is a multi-faceted response involving damage sensors, cell cycle checkpoints, and DNA repair proteins (1). Cells are also programmed with the ability to induce apoptosis if DNA damage is not able to be repaired (2). While many proteins have been identified that participate in the DNA damage response, the coordination and exact mechanism of the response remains to be elucidated. It is however apparent that the response to DNA damage is crucial for safeguarding the genome and avoiding neoplasia $(3,4)$.

\section{Cell Cycle Control}

Progression through the cell cycle contains a number of checkpoints that serve as quality control for accurate replication of DNA and synthesis of proteins required for cell division. This phenomenon was first described as a control mechanism enforcing dependency in the cell cycle (5). This definition has evolved over time as more functions of the cell cycle checkpoint have been elucidated. Now more commonly referred to as the DNA damage checkpoint, it serves as a control for DNA repair and replication through delay of the progression through the cell cycle. While the exact sensory mechanism remains a mystery, checkpoint activation can occur almost immediately after DNA is damaged (1).

The cell cycle represents the various stages by which a single cell is able to multiply into two daughter cells via the synthesis of the required proteins and complete 
replication of DNA. Newly divided cells begin with a resting or G0 phase, followed by G1, S, G2 and M. In the G1 phase cells are activating genes involved in the synthesis of deoxynucleotides and histone proteins as well as forming protein complexes at origins of replication to ensure proper initiation of replication. Much of G1 progress is in response to mitogenic stimuli. A checkpoint exists near the end of G1 before cells can progress into S phase. During S phase, cells replicate DNA in order to have 2 complete copies for subsequent cell division. Any damage incurred during this replication results in activation of the S phase checkpoint allowing time for the DNA repair pathway to deal with the lesion (6). The $\mathrm{S}$ phase checkpoint can be activated in a number of ways when the cell encounters difficulty with DNA replication. After passing through S phase, cells enter the G2 phase to ensure that DNA has been accurately replicated and that all the proteins required before segregation have been synthesized. Once the cell determines that all the correct components are in place, it will pass through the G2 checkpoint into M phase where the chromosomes segregate and the cell divides into two daughter cells. Any damage to chromosomes at this point activates the checkpoint and prevents the cell from undergoing mitosis while attempting to repair the damage.

Central to the control of cell cycle checkpoints are a set of molecules known as cell division cycle dual specificity phosphatases which includes Cdc25A, Cdc25B and Cdc25C. During the G1 phase, Cdc25A is an important signal mediator that serves as an inhibitor of the downstream signals that drive cell cycle progression thereby controlling early cell cycle progression (7-9). Later in the cell cycle, during G2 and M phases, $\mathrm{Cdc} 25 \mathrm{~B}$ and $\mathrm{Cdc} 25 \mathrm{C}$ act as signal mediators of cell cycle progression by control of the effector molecules (10). This simplified model of $\mathrm{Cdc} 25$ regulation has recently been 
brought into doubt. A number of recent studies have outlined roles for the $\mathrm{Cdc} 25$ proteins that are not cell cycle specific, suggesting that all three isoforms may coordinate to regulate cell cycle control (11-14). As this signaling pathway develops, it is clear that the Cdc25 family of phosphatases play a role in regulation of the effector molecules that control cell cycle progression.

This set of effector molecules known as cyclins and cyclin-dependent kinases (CDKs) function in response to upstream signals to drive cells from one stage of the cell cycle to the next when the correct conditions are met (15-17). An important upstream regulator in the control of cell cycle is the transcriptional repressor, $\mathrm{Rb}$. The binding to the E2F transcription factor and recruitment of histone deacetylase repressors allows $\mathrm{Rb}$ to halt many of the genes required for synthesis of DNA $(18,19)$. Disruption of this interaction by phosphorylation of $\mathrm{Rb}$ by $\mathrm{CDKs}$ allows $\mathrm{E} 2 \mathrm{~F}$ to activate the numerous genes required for synthesis of DNA during S phase $(20,21)$.

As our knowledge increased, it became more apparent that cyclins and CDKs were the machinery that controls the cell cycle checkpoints, but they did so in response to upstream signals that respond to conditions within the cell. Several of these proteins were discovered in response to DNA damage inflicted on the cell and they became part of a signaling pathway termed the DNA damage response. This was in contrast to the simple notion that a checkpoint was the main cellular response to DNA damage. It began to link both checkpoint control and DNA repair as part of the same process. Investigators began to see that the cell cycle checkpoint was only the brakes and the same proteins that activated the checkpoint were also able to activate DNA repair pathways (22-26). 


\section{DNA Repair}

There are several mechanisms both endogenous and exogenous by which DNA can be damaged in a cell and the repair pathway activated depends on the type of damage induced which is a direct result of the specific damaging agent. Ultraviolet (UV) light exposure leads mostly to pyrimidine dimers and other bulky adducts which are repaired by the nucleotide excision repair (NER) pathway (27). Oxygen radicals cause non-bulky base modifications repaired by the base excision repair (BER) pathway. Chemotherapeutic agents such as cisplatin or mitomycin $\mathrm{C}$ cause interstrand crosslinks (ICLs) repaired by recombinational repair, either NHEJ, HR or a combination of both as well as nucleotide excision repair (NER) (27). Ionizing radiation or stalled replication forks cause double strand breaks (DSBs) which can also be repaired by either NHEJ, HR or both in addition to NER $(27,28)$. This evidence brings to light the fact that multiple pathways respond to different types of DNA damage. Some of these differences can be attributed to the specific phase of cell cycle when the damage occurs. The rest is based largely on the type of damage that needs to be repaired.

While non-homologous end joining and homologous recombination both repair DSBs, the similarities in mechanism are few. NHEJ is the dominant repair mechanism during the early stages of the cell cycle where the availability of an undamaged template does not exist. A repair process which has no template for repair is more prone to error and often results in the addition or deletion of a few nucleotides. NHEJ relies on small sequence similarities between two broken strands to join the broken ends facilitated by a 
heterodimer complex of KU70 and KU80. After the ends are brought together they are ligated by DNA ligase 4 to complete the repair process (27).

The DNA damage trigger and some of the signals involved may be the same, but homologous recombination utilizes a vastly different mechanism for the repair of DSBs. The HR repair process dominates the later stages of S phase as well as G2. This could be merely due to the coincidence of the availability of a sister chromatid to use as a template for repair. The first step in HR is the resection of the DNA ends by nucleases followed by coating of the single strand of DNA by Rad51 which acts as a mammalian DNA recombinase similar to the bacterial protein $\operatorname{Rec} A(29,30)$. Rad51 facilitates the search for the undamaged template followed by the exchange of the single strand with the double strand forming a intermediate repair complex (30-32). The undamaged double strand is used as a template for DNA synthesis to repair both strands from the original lesion resulting in the formation of Holliday junctions which are quickly resolved into two double strands free of replication errors (33). Along with the proteins that have shown to be actively involved in the repair process, there are a number of other molecules that are recruited to the sites of DNA repair including, ATM/ATR, BRCA1, BRCA2 and H2AX. All have been shown to be recruited to the sites of DSBs and involved in the repair process even though their exact contribution is not yet well defined.

One of the early proteins activated in response to DSBs is the histone protein H2AX. Rapid phosphorylation of H2AX occurs at sites of DSBs on serine 139 resulting in the formation of discrete nuclear foci $(34,35)$. The phosphorylated form of $\mathrm{H} 2 \mathrm{AX}$, termed $\gamma$-H2AX, represents the catalytically active form of the histone protein first identified by two-dimensional gel electrophoresis after exposure to ionizing radiation 
(34). It was found that massive amounts of $\gamma-\mathrm{H} 2 \mathrm{AX}$ was found in complex with large amounts of chromatin at sites of DSBs in what became known as nuclear foci (35). The phosphorylation of H2AX was also implicated in the response to DSBs in an ATM dependent manner which would provide an eventual link to the DNA damage response (36). Evidence later showed that the recruitment of several of these repair factors into nuclear foci is dependent on $\gamma-\mathrm{H} 2 \mathrm{AX}$ as well as members of the PI-3 kinase-like family such as ATM and ATR (37). It has been noted in several recent reports that fluorescent staining for $\gamma-\mathrm{H} 2 \mathrm{AX}$ serves as a sensitive method of detection for DSBs $(34,37-40)$. A number of other proteins involved in DNA repair and cell cycle control including BRCA1, MRE11, Rad50 and Rad51 were subsequently shown to be part of the nuclear foci at sites of DSBs and are involved in the various functions of the DNA damage response (41-44).

Generally regarded as the apical protein in the DNA damage signaling network, ATM was first identified as the gene product mutated in the genetic disorder ataxia telangiectasia (AT) (45). This is a rare genetic disorder characterized by defective response to specific types of DNA damage, hypersensitivity to IR and genomic instability. Characteristics of the disease point to a defect in response to agents that cause DSBs. It was subsequently shown that cells cultured from AT patients lack the ability to repair DSBs (46). Loss of ATM function is responsible for an increase risk of developing certain types of cancer $(46,47)$ Later, another protein was cloned that showed significant homology to ATM and was thus termed, ATR (ATM and Rad3 related) (48).

ATM and ATR are PI3K like proteins that act as serine-threonine kinases early in the DNA damage response pathway playing a central role in transmitting signals to 
downstream proteins via phosphorylation $(46,47,49-51)$. They contain similar structure and domains as PI3K and have been shown to activate specific checkpoint control functions of BRCA1 $(22,52)$. Evidence suggests that ATM primarily responds to DSBs caused by IR, while ATR reacts to UV induced damage as well as several other types of lesions or bulky adducts (53). Evidence also exists that point to chromatin alterations as a mechanism for activation of ATM which suggests a possible role as a sensor of DNA damage (54). While both have been speculated to be damage sensors, they have definitively been shown to transduce the damage signal early on in the pathway to multiple downstream effector proteins including p53, CHK2, H2AX, NBS1, BRCA1 and FANCD2 $(47,51)$. This represents a major branch point in DNA damaging signaling based on the broad number of ATM substrates and the diverse cellular functions that they control. The involvement of ATM in activation of transcription has been noted by involvement in the activation of $\mathrm{p} 53$ and $\mathrm{E} 2 \mathrm{~F} 1$, both of which control numerous functions relating to cell cycle, apoptosis and DNA repair (55-58). Recent work using large scale proteomic analysis identified over 900 phosphorylation motifs in 700 proteins that were induced in response to IR highlighting the large network of proteins involved in the DNA damage response (59).

The ATM protein contains three distinct domains; FAT, FATC and PI3K. The functional singinfcance of the FAT and FATC are not clearly defined while the PI3K domain contains the catalytic site for kinase activity. ATM activity in response to IR is regulated by auto-phosphorylation on serine 1981 which is located within the FAT domain (47). Inactive ATM is present as homodimers in the nucleus that are subsequently activated upon IR induced DSBs $(56,57)$. This auto-phosphorylation mechanism 
facilitates dimer dissociation and causes a rapid increase in ATM activity that initiates its kinase activity for a number of substrates including $\operatorname{BRCA} 1(54,60)$.

First identified as an inherited protein that contributes to increased risk of developing breast or ovarian cancer (61), BRCA1 has been heavily studied in the last decade and much has been determined about its specific functions. Acting as a tumor suppressor, BRCA1 is frequently mutated in familial breast and ovarian cancers $(62,63)$. Nearly $10 \%$ of women with breast or ovarian cancer and $50 \%$ of inherited breast and ovarian cancers can be attributed to a mutation in the BRCA1 gene $(63,64)$. BRCA1 plays a key role in the radiation induced DNA damage response and is involved in multiple cellular processes including cell cycle checkpoint control, chromosome remodeling, transcriptional regulation, DNA repair and apoptosis $(44,63-65)$. Such a diverse list of cellular functions relating to DNA damage places BRCA1 as a central molecule in the DNA damage signaling network highlights its importance as a tumor suppressor (66) (Figure 1). Several studies have noted a modulation of sensitivity to DNA damaging agents based on BRCA1 status including IR and cisplatin (67-73). 
Figure 1. Aspects of the DNA damage response dependent on BRCA1

DNA Damage

Response

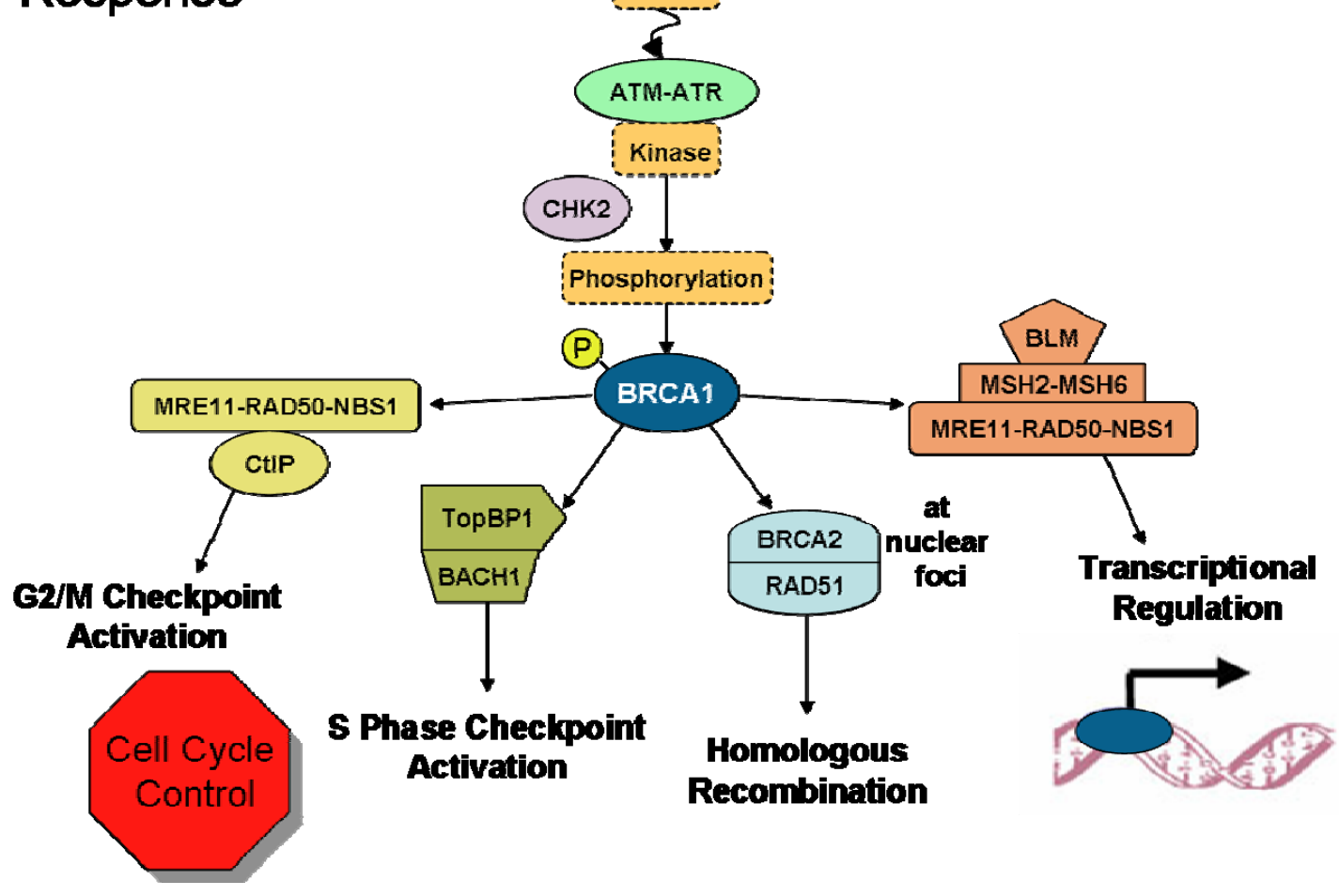


The structure and molecular interactions of BRCA1 reveal its ability to interact with a number of binding partners and control numerous processes. BRCA1 is a 1863 amino acid protein and the N-terminus contains a RING finger domain, from amino acids 8-96, often associated with E3 ubiquitin ligase activity which has been shown to occur in a BRCA1 dependent manner in conjunction with BARD1 $(61,74,75)$. The N-terminal domain also contains a site for interaction with E2F1 (76). Adjacent to the RING domain, BRCA1 contains two nuclear localization signals between amino acids 200 and 300 which also contains a binding site for $\mathrm{Rb}$ and c-Myc $(77,78)$. The central portion of the protein contains several serine residues that are important in regulation by phosphorylation as well as binding sites for $\operatorname{Rad} 51, \mathrm{ATM}$ and $\mathrm{CHK} 2(22,79,80)$. The phosphorylation sites are called the SQ cluster referring to the serine residue that is actually phosphorylated followed by a glutamine residue. Early evidence revealed a role for ATM and ATR in phosphorylation of distinct sites of BRCA1 after exposure to either UV or IR (52). Serine residues 1387 and 1423 of BRCA1 have been shown to be phosphorylated by ATM/ATR in response to DNA damage and subsequently control the $\mathrm{S}$ and $\mathrm{G} 2 / \mathrm{M}$ checkpoints $(22,52)$. Exposure to UV or ionizing radiation leads to phosphorylation of Ser-1423 and Ser-1524 $(22,52)$. In contrast, only UV radiation is able to induce phosphorylation of Ser-1457 while IR specifically induces phosphorylation of Ser-1387 (52). Studies also revealed that BRCA1, in conjunction with ATM, participated in the control of both $\mathrm{S}$ and $\mathrm{G} 2$ checkpoints in response to IR $(81,82)$. This study highlighted the requirement for phosphorylation of Ser-1423 for control of the G2 checkpoint (81). Follow up to this study linked S phase checkpoint control to specific phosphorylation of Ser-1387 after ionizing radiation (83). This evidence was later 
developed into a model whereby differential phosphorylation of BRCA1 on either Ser 1387 or Ser-1423 by ATM and/or ATR played a pivotal role in cell cycle control after DNA damage.

The C-terminal end contains two BRCT (BRCA1 C-Terminal Domain) domains that interact with a number of proteins important for transcription, cell cycle control and DNA repair including p53, Rb, RNA Pol II, RNA Helicase A, Rad51 and BRCA2 (8490). These interactions were an important clue that provided evidence linking BRCA1 to DNA repair. The requirement of the BRCT domains for many of the functions of BRCA1 became increasingly evident as more was revealed about the proteins that interact in this region as well as the revelation that is a phospho-protein binding site highly involved in the DNA damage response (91-93). The disruption of these interactions became a highly important tool and provided a molecular basis for development of a model system from a tumor cell line, designated HCC1937, that was established from a grade III infiltrating ductal primary breast tumor from a breast cancer patient with a germ-line BRCA1 mutation (94). The loss of interaction with proteins in the BRCT domains rendered BRCA1 non-functional in these cells adversely affecting the DNA damage response (95). Stable expression of wild type BRCA1 in these cells is the mostly widely used model for assessment of BRCA1 functions in response to DNA damage (95).

Initial evidence indicating hyperphosphorylation and relocation of BRCA1 after DNA damage provided the first observational clue for a role for BRCA1 in DNA repair $(44,65)$. BRCA1 is active in the repair of damaged DNA by interaction with proteins in the transcription-coupled nucleotide excision repair (TC-NER), NHEJ and HR pathways (96-99). The precise role that BRCA1 plays in DNA repair remains unknown, but it has 
been shown to interact specifically with repair proteins $\operatorname{Rad} 50, \operatorname{Rad} 51, \mathrm{H} 2 \mathrm{AX}$ and BRCA2 $(79,89,100)$. Not only have these repair proteins been shown to interact, but they also colocalize to discrete nuclear foci after treatment with DNA damaging agents $(37,44)$. One study highlighted a super complex of proteins assembled in response to DNA damage termed the BRCA1-associated genome surveillance complex (BASC) which included MSH2, MSH6, MLH1, ATM, BLM, and the RAD50-MRE11-NBS1 protein complex (101).

A transcriptional role for BRCA1 creates another scenario by which BRCA1 plays a central role in the DNA damage response. The first evidence of the transcriptional activity of BRCA1 came from the observation that the C-terminal region of BRCA1 fused to a GAL4 binding domain activated transcription of several GAL4 dependent promoters and mutations in this region of BRCA1 abrogated this transcription $(102,103)$. This evidence was followed up by studies that showed that BRCA1 forms a complex with RNA polymerase II thus affecting transcription $(90,104)$. Even more evidence for the role of BRCA1 in transcription came from a report that showed the ability of BRCA1 to interact with p53 and stimulate transcription from the p21 promoter (86). Evidence also exists for an interaction with c-Myc oncogene with is a transactivator for the transcription of several genes (78).

CHK2 is a checkpoint control protein that is another known substrate of ATM and operates in response to DNA damage by regulating both the G1 and G2 checkpoints by activation of proteins such as p53, E2F1, CDC25A and BRCA1 (105-109). Induction of DNA damage leads to activation of ATM by IR as well as subsequent phosphorylation of CHK2 on Threonine 68 (110). Regulation of the G2/M checkpoint 
requires functional $\mathrm{CHK} 2$ as evidenced by defects in checkpoint regulation observed in CHK2 -/- embryonic stem cells (106). CHK2 is able to phosphorylate BRCA1 on serine 988 and is also shown to interact with BRCA1 at discrete nuclear foci after IR exposure (107). These data indicate a signaling network that begins with initial activation of ATM followed by CHK2 and BRCA1 activation resulting in control of the G2/M checkpoint in response to DNA damage.

CHK2 has also been shown in conjunction with ATM/ATR to control the G1 checkpoint by control of p53 and E2F1. Studies have suggested a role for CHK2 dependent phosphorylation of $\mathrm{p} 53$ at Ser-20, a site critical for stabilization of $\mathrm{p} 53$ activity leading to activation of the G1 checkpoint (111-115). Phosphorylation of E2F1 by CHK2 at Ser-364 has been shown in response to DNA damage leading to stabilization and transcriptional activation of the protein (109). This is in agreement with data that shows that E2F1 deficient thymocytes are resistant to etoposide induced apoptosis (55).

Three distinct domains make up the CHK2 protein, an SQ cluster domain, forkhead associated domain and a kinase domain (116). The SQ cluster domain contains a number of residues that are possible phosphorylation targets for ATM (117). Inactive CHK2 exists as a monomer before activation by DNA damage when it undergoes dimerization with another molecule of CHK2 (118-120). Dimerization is then followed by a number of intermolecular phosphorylation events in the auto-inhibitory portion of the molecule leading to activation (121-123). The signal is then relayed to the downstream cell division cycle dual specificity phosphatase family, which includes Cdc25A and Cdc25C. The Cdc25 family directly promotes cell cycle progression by 
activation of the cyclin dependent kinases, the functional control molecules of the cell cycle $(10,105,108,124,125)$.

\section{Apoptosis}

One characteristic in the development of cancer is the loss of regulation of cell death (126). A balance exists where the rate of cell division and rate of cell death via apoptosis is able to regulate homeostasis within a tissue. Disruption of apoptosis alters the rate of cell death leading to the ability to increase cell numbers with little or no control (126). This is a contributing factor in tumor development (127). A common strategy to combat tumor growth is to circumvent the loss of apoptosis by stimulating the pathway via an alternate mechanism (128). The reality is that while this approach may work in some cases, tumors often may not respond to such treatments or may easily become resistant. For this reason, it is important to understand what signals these agents use to activate apoptosis in order to determine types of tumors that may respond more robustly to specific chemotherapeutics.

Caspase dependent apoptosis is a form of programmed cell death controlled by a pathway of cysteine proteases known as caspases. It is characterized by shrinkage in overall cellular size, condensation of chromatin and DNA fragmentation (129). The structure of caspase family members is similar in that most contain pro-domains as well as a small and large subunit to make the full length single chain protein (130). They are synthesized as inactive zymogens and enzymatic activation is achieved through cleavage at specific sites in the $\mathrm{N}$-terminal, after an aspartic acid residue $(130,131)$. Active caspases are able to cleave numerous target proteins involved in cytoskeletal and nuclear 
structure effectively controlling the execution stage characterized by dismantling of the cell (132-135). Cleavage is often carried out by an upstream protease with or without dependence on co-factors and can also occur autocatalytically (129). This mechanism of action can serve as a feedback loop to quickly and exponentially increase the amount of active enzyme in a cell. 
Figure 2. Simplified overview of caspase involvement in control of apoptosis in response to DNA damage.

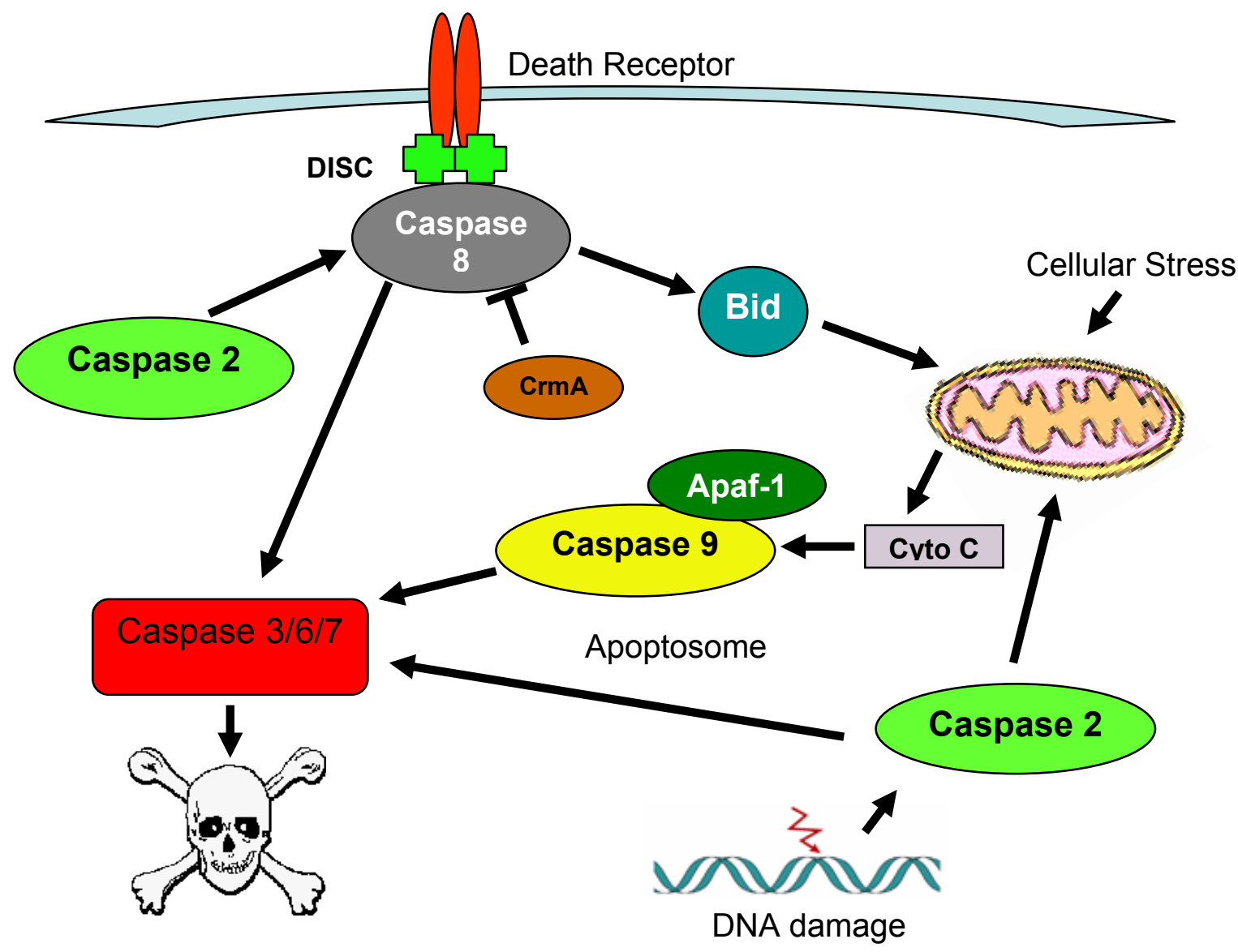


Both an intrinsic and extrinsic pathway of activation exists, merging with the activation of the effector caspases 3,6 and 7. Initiator caspases 2, 8 and 9 respond to different stimuli and regulate the activity of downstream effectors. Caspase 2 responds predominantly to genotoxic stress via an unknown mechanism. Caspase 8 is mostly activated by extracellular death receptors, while caspase 9 responds to intracellular stress signaled through the mitochondria.

Caspase 2 is one of the earliest identified caspases in mammals and appears to be required for induction of apoptosis triggered by DNA damage (136). Recent evidence has linked PIDD (p53-induced protein with a death domain) in the formation of large multimeric protein complexes with caspase 2 called a PIDDosome that can lead to activation of caspase 2 and sensitize cells to genotoxic stress (137). Caspase 2 is unique in that the structure and sequence homology are closely related to that of the other initiator caspases while the cleavage specificity is more closely related to executioner caspases 3 and 7 (138). Several reports have shown caspase 2 to be activated upstream of caspase 9 and cytochrome $\mathrm{c}$ release (139-141), including a study revealing that caspase 2 is able to cause mitochondrial dysfunction while still in the nucleus, resulting in cytochrome c release, possibly though the truncation of Bid (139). This data supports a role for caspase 2 upstream of the mitochondria and a requirement for caspase 9 (141), while other evidence implicates a role for caspase 2 unrelated to mitochondrial activity. One study reported the recruitment of procaspase 2 into a multimeric complex which is sufficient to activate it in a manner independent of mitochondrial activity or cofactors such as cytochrome c and Apaf-1 (142). This line of evidence supports a mechanism in which caspase 2 alone is able to induce apoptosis whereas subsequent downstream 
cytochrome c release and caspase 9 activation may act as an independent amplification loop (142). Evidence also exists demonstrating the ability of caspase 8 to be activated downstream of caspase 2 during ceramide or etoposide induced apoptosis (143). While no definitive answer currently exists, it is clear that caspase 2 is important for signaling of apoptosis induced by DNA damage.

The death receptor mediated pathway responds to extracellular signals which are able to activate apoptosis. Tumor necrosis factor receptor (TNFR) and Fas are two examples of death receptors that are well characterized and have a distinct cytoplasmic domain known as the death domain (144). After receptor engagement, the cytoplasmic domain of the receptor is able to bind a number of FADD (Fas-associated death domain) adaptor molecules through homotypic interaction between the death domain (DD) of both Fas and FADD $(145,146)$. The N-terminal portion of FADD contains a death effector domain (DED) that acts to recruit caspase 8 to the complex and forms an interaction with the DED of procaspase $8(145,147)$. This interaction between Fas, FADD and procaspase 8 results in formation of a large complex known as the death-inducing signaling complex (DISC) (148). Oligomerization of procaspase 8 with adaptor molecules at receptor sites leads to autoproteolytic cleavage of caspase 8 and subsequent activation of effector caspases $(149,150)$. A recent report has suggested the ability of caspase 2 to act as a priming mechanism for receptor induced apoptosis. In this model, caspase 2 is able to cleave procaspase 8 leaving it primed for recruitment to the DISC after receptor engagement where oligomerization can lead to full activation (151). Evidence also exists demonstrating the ability of caspase 8 to cleave Bid resulting in release of cytochrome $\mathrm{c}$ and activation of the mitochondrial mediated pathway (152). 
The mitochondrial mediated pathway activates apoptosis in response to cellular stress regulated by a number of pro and anti-apoptotic proteins. This extensive array of proteins modulates the membrane potential of the mitochondria, controlling release of cytochrome $\mathrm{c}$ into the cytoplasm which leads to the formation of the activated apoptosome complex of caspase 9 and Apaf-1 (153). Apaf-1 acts likes a scaffolding molecule and forms a complex with caspase 9 at a 1:1 ratio followed by oligomerization in a larger mutlimeric complex called the apoptosome which leads to autoproteolytic cleavage (154). Active caspase 9 is then able to cleave and activate the effector caspase 3 (155).

Effector caspases activate a number of proteins involved in disassembly of the cell such as lamins, gelsolin, focal adhesion kinase (FAK) and PARP (132-135). Many are cytoskeletal proteins that are disrupted allowing for cellular disassembly. Nuclear lamina forms polymers that provide structure for chromatin. When lamins are cleaved during apoptosis, this structure is lost causing condensation of chromatin (132). Cytoskeletal rearrangement is also very pronounced in apoptosis, regulated by cleavage of cytoskeletal proteins such as gelsolin and FAK $(133,134)$. PARP is a very important substrate for the caspase cascade that is involved in DNA repair and contributes to genomic stability (156). Cleavage of PARP disrupts genomic stability leading to DNA fragmentation that is a hallmark of apoptosis.

\section{Nonapoptotic role of caspases}

Recent mounting evidence has linked several members of the caspase family to nonapoptotic cellular roles including embryonic development, macrophage 
differentiation, $\mathrm{T}$ cell proliferation and cell motility (157-164). Several of these examples include diverse cellular functions and signals. The differentiation of monocytes into mature macrophages during the process of infection occurs in the presence of several active caspases while not exhibiting the morphological characteristics that are a hallmark of apoptosis (161). In this study, caspase activity seemed to drive differentiation while zVAD, a chemical inhibitor of apoptosis, was able to block this maturation process and resensitize the cells to apoptosis (161). Other evidence also revealed a mechanism by which caspase 3 was able to drive platelet formation in the absence of detectable DNA fragmentation (165). Interestingly, caspase 3 showed a punctuate cytoplasmic staining during this process as opposed to the diffuse staining seen during the induction of apoptosis by staurosporine (165). The pan-caspase inhibitor z-VAD was able to block platelet formation as was more specific inhibitors for caspases 3 and 9 (165).

Another interesting example of a nonapoptotic role for caspases is in cell motility in caspase 8 null mouse embryo fibroblasts. This set of experiments outlined a role for caspase 8 in increased cell motility under nonapoptotic conditions (158). This phenomenon was linked to caspase 8 dependent calpain activity and was corroborated by evidence using the viral crmA inhibitor of caspase 8 (158). There is no doubt that alternate roles for caspases are emerging in this rapidly expanding field.

\section{Irofulven}

Irofulven (6-hydroxymethylacylfulvene, MGI 114, NSC\#: 683863) is one of a new class of anticancer agents that are analogs of the mushroom derived illudin $\mathrm{S}$ toxins. Preclinical and clinical trials have shown that irofulven is effective against several solid 
tumor types (166-172). The exact mechanism of action of irofulven remains mostly unknown. While previous studies have suggested that damage induced by irofulven is repaired exclusively via the transcription coupled nucleotide excision repair (TC-NER) pathway, these studies have ignored the NHEJ and HR pathways (173). Recent work in our lab has shown that irofulven activates ATM and its downstream targets: NBS1, FANCD2, SMC1, CHK2 and p53 (174-176).

Studies on the activation of apoptotic pathway by DNA damaging agents makes the case that these agents provide a strong mechanism for the induction of apoptosis (136). Although most data shows that irofulven is able to cause DNA damage, the precise mechanism of killing remains largely unknown. Previous studies have shown induction of apoptosis mediated by ERK and JNK kinases (177). It has also been shown that irofulven treatment induces the cleavage of caspases 8 and 9 (178). This data has been corroborated by other evidence that caspase induced apoptosis plays a role in cell death after irofulven treatment (179).

\section{Summary and Significance}

There are several first line cancer therapeutics, as well as ionizing radiation, that rely on the ability to damage DNA to kill cells. Some of these DNA damaging agents have been shown to cause inter-strand cross links or double strand breaks while others cause stalled replication that results in cell death. Although the nature of the damage induced may be different, DNA damaging agents remain some of the most effective therapeutics available. Based on the damage inflicted, each agent activates a unique 
response. The ability of cancers to evade these commonly used drugs makes it necessary to explore new therapies that target the cancer cells using different mechanisms as well as to treat tumors that develop resistance. Irofulven represents a novel DNA damaging agent that provides useful insight into the DNA damage response pathway. A unique pathway, activated by a novel damaging agent proves useful in elucidating more about how tumor cells respond and the proteins involved. 
1. Zhou, B. B. and Elledge, S. J. The DNA damage response: putting checkpoints in perspective. Nature, 408: 433-439, 2000.

2. Clarke, A. R., Purdie, C. A., Harrison, D. J., Morris, R. G., Bird, C. C., Hooper, M. L., and Wyllie, A. H. Thymocyte apoptosis induced by p53-dependent and independent pathways. 362: 849-852, 1993.

3. Bartkova, J., Horejsi, Z., Koed, K., Kramer, A., Tort, F., Zieger, K., Guldberg, P., Sehested, M., Nesland, J. M., Lukas, C., Orntoft, T., Lukas, J., and Bartek, J. DNA damage response as a candidate anti-cancer barrier in early human tumorigenesis. 434: 864-870, 2005.

4. Hartwell, L. H. and Kastan, M. B. Cell cycle control and cancer. Science, 266: 1821-1828, 1994.

5. Hartwell, L. H. and Weinert, T. A. Checkpoints: controls that ensure the order of cell cycle events. Science, 246: 629-634, 1989.

6. Bartek, J., Lukas, C., and Lukas, J. CHECKING ON DNA DAMAGE IN S PHASE. Nature Reviews Molecular Cell Biology, 5: 792-804, 2004.

7. Jinno, S., Suto, K., Nagata, A., Igarashi, M., Kanaoka, Y., Nojima, H., and Okayama, H. Cdc25A is a novel phosphatase functioning early in the cell cycle. Embo J, 13: 1549-1556, 1994. 
8. Hoffmann, I., Draetta, G., and Karsenti, E. Activation of the phosphatase activity of human cdc25A by a cdk2-cyclin E dependent phosphorylation at the G1/S transition. Embo J, 13: 4302-4310, 1994.

9. Sexl, V., Diehl, J. A., Sherr, C. J., Ashmun, R., Beach, D., and Roussel, M. F. A rate limiting function of cdc25A for S phase entry inversely correlates with tyrosine dephosphorylation of Cdk2. Oncogene, 18: 573-582, 1999.

10. Morgan, D. O. Principles of CDK regulation. Nature, 374: 131-134, 1995.

11. Lindqvist, A., Kallstrom, H., Lundgren, A., Barsoum, E., and Rosenthal, C. K. Cdc25B cooperates with Cdc25A to induce mitosis but has a unique role in activating cyclin B1-Cdk1 at the centrosome. J. Cell Biol., 171: 35-45, 2005.

12. Mailand, N., Podtelejnikov, A., Groth, A., Mann, M., Bartek, J., and Lukas, J. Regulation of G2/M events by Cdc25A through phosphorylation-dependent modulation of its stability. Embo J, 21: 5911-5920, 2002.

13. Busino, L., Donzelli, M., Chiesa, M., Guardavaccaro, D., Ganoth, D., Dorrello, N. V., Hershko, A., Pagano, M., and Draetta, G. F. Degradation of Cdc25A by betaTrCP during S phase and in response to DNA damage. Nature, 426: 87-91, 2003.

14. Donzelli, M., Squatrito, M., Ganoth, D., Hershko, A., Pagano, G., and Draetta, G. Dual mode of degradation of Cdc25 A phosphatase. Embo J, 21: 4875-4884, 2002. 
15. Pardee, A. B. G1 events and regulation of cell proliferation. Science, 246: 603$608,1989$.

16. Xiong, Y., Connolly, T., Futcher, B., and Beach, D. Human D-type cyclin. Cell, 65: 691-699, 1991.

17. Matsushime, H., Roussel, M. F., Ashmun, R. A., and Sherr, C. J. Colonystimulating factor 1 regulates novel cyclins during the G1 phase of the cell cycle. Cell, 65: 701-713, 1991.

18. Nevins, J. R. E2F: a link between the Rb tumor suppressor protein and viral oncoproteins. Science, 258: 424-429, 1992.

19. Brehm, A., Miska, E. A., McCance, D. J., Reid, J. L., Bannister, A. J., and Kouzarides, T. Retinoblastoma protein recruits histone deacetylase to repress transcription. 391: 597-601, 1998.

20. Zhang, H. S., Gavin, M., Dahiya, A., Postigo, A. A., Ma, D., Luo, R. X., Harbour, J. W., and Dean, D. C. Exit from G1 and S Phase of the Cell Cycle Is Regulated by Repressor Complexes Containing HDAC-Rb-hSWI/SNF and Rb-hSWI/SNF. Cell, 101: 79-89, 2000.

21. Harbour, J. W., Luo, R. X., Santi, A. D., Postigo, A. A., and Dean, D. C. Cdk Phosphorylation Triggers Sequential Intramolecular Interactions that Progressively Block Rb Functions as Cells Move through G1. Cell, 98: 859-869, 1999. 
22. Cortez, D., Wang, Y., Qin, J., and Elledge, S. J. Requirement of ATM-dependent phosphorylation of brcal in the DNA damage response to double-strand breaks. Science, 286: 1162-1166, 1999.

23. Lim, D. S., Kim, S. T., Xu, B., Maser, R. S., Lin, J., Petrini, J. H., and Kastan, M. B. ATM phosphorylates p95/nbs1 in an S-phase checkpoint pathway. Nature, 404: 613-617, 2000.

24. Gatei, M., Young, D., Cerosaletti, K. M., Desai-Mehta, A., Spring, K., Kozlov, S., Lavin, M. F., Gatti, R. A., Concannon, P., and Khanna, K. ATM-dependent phosphorylation of nibrin in response to radiation exposure. Nat Genet, 25: 115$119,2000$.

25. Zhao, S., Weng, Y. C., Yuan, S. S., Lin, Y. T., Hsu, H. C., Lin, S. C., Gerbino, E., Song, M. H., Zdzienicka, M. Z., Gatti, R. A., Shay, J. W., Ziv, Y., Shiloh, Y., and Lee, E. Y. Functional link between ataxia-telangiectasia and Nijmegen breakage syndrome gene products. Nature, 405: 473-477, 2000.

26. Wu, X., Ranganathan, V., Weisman, D. S., Heine, W. F., Ciccone, D. N., O'Neill, T. B., Crick, K. E., Pierce, K. A., Lane, W. S., Rathbun, G., Livingston, D. M., and Weaver, D. T. ATM phosphorylation of Nijmegen breakage syndrome protein is required in a DNA damage response. Nature, 405: 477-482, 2000.

27. Hoeijmakers, J. H. Genome maintenance mechanisms for preventing cancer. Nature, 411: 366-374, 2001. 
28. Ward, I. and Chen, J. Early Events in the DNA Damage Response. In: Current Topics in Developmental Biology, Volume 63 edition, pp. 1-35: Academic Press, 2004.

29. Paull, T. T. and Gellert, M. The 3' to 5' Exonuclease Activity of Mre11 Facilitates Repair of DNA Double-Strand Breaks. Molecular Cell, 1: 969-979, 1998.

30. Shinohara, A., Ogawa, H., and Ogawa, T. Rad51 protein involved in repair and recombination in S. cerevisiae is a RecA-like protein. Cell, 69: 457-470, 1992.

31. Baumann, P., Benson, F. E., and West, S. C. Human Rad51 Protein Promotes ATP-Dependent Homologous Pairing and Strand Transfer Reactions In Vitro. Cell, 87: 757-766, 1996.

32. Sung, P. Catalysis of ATP-Dependent Homologous DNA Pairing and Strand Exchange by Yeast RAD51 Protein. Science, 265: 1241-1243, 1994.

33. Khanna, K. K. and Jackson, S. P. DNA double-strand breaks: signaling, repair and the cancer connection. Nat Genet, 27: 247-254, 2001.

34. Rogakou, E. P., Pilch, D. R., Orr, A. H., Ivanova, V. S., and Bonner, W. M. DNA Double-stranded Breaks Induce Histone H2AX Phosphorylation on Serine 139. J. Biol. Chem., 273: 5858-5868, 1998.

35. Rogakou, E. P., Boon, C., Redon, C., and Bonner, W. M. Megabase Chromatin Domains Involved in DNA Double-Strand Breaks In Vivo. J. Cell Biol., 146: 905-916, 1999. 
36. Burma, S., Chen, B. P., Murphy, M., Kurimasa, A., and Chen, D. J. ATM Phosphorylates Histone H2AX in Response to DNA Double-strand Breaks. J. Biol. Chem., 276: 42462-42467, 2001.

37. Paull, T. T., Rogakou, E. P., Yamazaki, V., Kirchgessner, C. U., Gellert, M., and Bonner, W. M. A critical role for histone H2AX in recruitment of repair factors to nuclear foci after DNA damage. Curr Biol, 10: 886-895, 2000.

38. Sedelnikova, O. A., Rogakou, E. P., Panyutin, I. G., and Bonner, W. M. Quantitative Detection of 125IdU-Induced DNA Double-Strand Breaks with \&gamma;-H2AX Antibody. Radiation Research 486-492, 2002.

39. Rothkamm, K. and Lobrich, M. Evidence for a lack of DNA double-strand break repair in human cells exposed to very low x-ray doses. PNAS, 100: 5057-5062, 2003.

40. Franco, S., Gostissa, M., Zha, S., Lombard, D. B., Murphy, M. M., Zarrin, A. A., Yan, C., Tepsuporn, S., Morales, J. C., Adams, M. M., Lou, Z., Bassing, C. H., Manis, J. P., Chen, J., Carpenter, P. B., and Alt, F. W. H2AX Prevents DNA Breaks from Progressing to Chromosome Breaks and Translocations. Molecular Cell, 21: 201-214, 2006.

41. Maser, R. S., Monsen, K. J., Nelms, B. E., and Petrini, J. H. hMre11 and hRad50 nuclear foci are induced during the normal cellular response to DNA doublestrand breaks. Mol Cell Biol, 17: 6087-6096, 1997. 
42. Haaf, T., Golub, E., Reddy, G., Readdin, C., and Ward, D. Nuclear Foci of Mammalian Rad51 Recombination Protein in Somatic cells After DNA Damage and its Localization in Synaptonemal Complexes. PNAS, 92: 2298-2302, 1995.

43. Raderschall, E., Golub, E. I., and Haaf, T. Nuclear foci of mammalian recombination proteins are located at single-stranded DNA regions formed after DNA damage. PNAS, 96: 1921-1926, 1999.

44. Scully, R., Chen, J., Ochs, R. L., Keegan, K., Hoekstra, M., Feunteun, J., and Livingston, D. M. Dynamic changes of BRCA1 subnuclear location and phosphorylation state are initiated by DNA damage. Cell, 90: 425-435, 1997.

45. Savitsky, K., Bar-Shira, A., Gilad, S., Rotman, G., Ziv, Y., Vanagaite, L., Tagle, D. A., Smith, S., Uziel, T., Sfez, S., and et al. A single ataxia telangiectasia gene with a product similar to PI-3 kinase. Science, 268: 1749-1753, 1995.

46. Shiloh, Y. and Kastan, M. B. ATM: genome stability, neuronal development, and cancer cross paths. Adv Cancer Res, 83: 209-254, 2001.

47. Shiloh, Y. ATM and related protein kinases: safeguarding genome integrity. Nat Rev Cancer, 3: 155-168, 2003.

48. Cimprich, K. A., Shin, T. B., Keith, C. T., and Schreiber, S. L. cDNA cloning and gene mapping of a candidate human cell cycle checkpoint protein. Proc Natl Acad Sci U S A, 93: 2850-2855, 1996. 
49. Shiloh, Y. ATM and ATR: networking cellular responses to DNA damage. Curr Opin Genet Dev, 11: 71-77, 2001.

50. Abraham, R. T. Cell cycle checkpoint signaling through the ATM and ATR kinases. Genes Dev, 15: 2177-2196, 2001.

51. Elledge, S. J. Cell Cycle Checkpoints: Preventing an Identity Crisis. Science, 274: 1664-1672, 1996.

52. Gatei, M., Zhou, B. B., Hobson, K., Scott, S., Young, D., and Khanna, K. K. Ataxia telangiectasia mutated (ATM) kinase and ATM and Rad3 related kinase mediate phosphorylation of Brcal at distinct and overlapping sites. In vivo assessment using phospho-specific antibodies. J Biol Chem, 276: 17276-17280, 2001.

53. Kastan, M. B. and Bartek, J. Cell-cycle checkpoints and cancer. Nature, 432: 316$323,2004$.

54. Bakkenist, C. J. and Kastan, M. B. DNA damage activates ATM through intermolecular autophosphorylation and dimer dissociation. Nature, 421: 499-506, 2003.

55. Lin, W.-C., Lin, F.-T., and Nevins, J. R. Selective induction of E2F1 in response to DNA damage, mediated by ATM-dependent phosphorylation. Genes Dev., 15: 1833-1844, 2001. 
56. Banin, S., Moyal, L., Shieh, S., Taya, Y., Anderson, C. W., Chessa, L., Smorodinsky, N. I., Prives, C., Reiss, Y., Shiloh, Y., and Ziv, Y. Enhanced phosphorylation of p53 by ATM in response to DNA damage. Science, 281: 1674-1677, 1998.

57. Canman, C. E., Lim, D. S., Cimprich, K. A., Taya, Y., Tamai, K., Sakaguchi, K., Appella, E., Kastan, M. B., and Siliciano, J. D. Activation of the ATM kinase by ionizing radiation and phosphorylation of p53. Science, 281: 1677-1679, 1998.

58. Khanna, K. K., Keating, K. E., Kozlov, S., Scott, S., Gatei, M., Hobson, K., Taya, Y., Gabrielli, B., Chan, D., Lees-Miller, S. P., and Lavin, M. F. ATM associates with and phosphorylates p53: mapping the region of interaction. Nat Genet, 20: 398-400, 1998.

59. Matsuoka, S., Ballif, B. A., Smogorzewska, A., McDonald, E. R., III, Hurov, K. E., Luo, J., Bakalarski, C. E., Zhao, Z., Solimini, N., Lerenthal, Y., Shiloh, Y., Gygi, S. P., and Elledge, S. J. ATM and ATR Substrate Analysis Reveals Extensive Protein Networks Responsive to DNA Damage. Science, 316: 1160$1166,2007$.

60. Lee, J. H. and Paull, T. T. Direct activation of the ATM protein kinase by the Mre11/Rad50/Nbs1 complex. Science, 304: 93-96, 2004.

61. Miki, Y., Swensen, J., Shattuck-Eidens, D., Futreal, P. A., Harshman, K., Tavtigian, S., Liu, Q., Cochran, C., Bennett, L. M., Ding, W., and et al. A strong 
candidate for the breast and ovarian cancer susceptibility gene BRCA1. Science, 266: 66-71, 1994.

62. Venkitaraman, A. R. Tracing the network connecting BRCA and Fanconi anaemia proteins. Nat Rev Cancer, 4: 266-276, 2004.

63. Narod, S. A. and Foulkes, W. D. BRCA1 and BRCA2: 1994 and beyond. Nat Rev Cancer, 4: 665-676, 2004.

64. Venkitaraman, A. R. Cancer susceptibility and the functions of BRCA1 and BRCA2. Cell, 108: 171-182, 2002.

65. Thomas, J., Smith, M., Tonkinson, J., Rubinfeld, B., and Polakis, P. Induction of phosphorylation on BRCA1 during the cell cycle and after DNA damage. Cell Growth Differ, 8: 801-809, 1997.

66. Scully, R. and Livingston, D. M. In search of the tumour-suppressor functions of BRCA1 and BRCA2. Nature, 408: 429-432, 2000.

67. Abbott, D. W., Thompson, M. E., Robinson-Benion, C., Tomlinson, G., Jensen, R. A., and Holt, J. T. BRCA1 expression restores radiation resistance in BRCA1defective cancer cells through enhancement of transcription-coupled DNA repair. J Biol Chem, 274: 18808-18812, 1999.

68. Le Page, F., Randrianarison, V., Marot, D., Cabannes, J., Perricaudet, M., Feunteun, J., and Sarasin, A. BRCA1 and BRCA2 are necessary for the 
transcription-coupled repair of the oxidative 8-oxoguanine lesion in human cells. Cancer Res, 60: 5548-5552, 2000.

69. Taniguchi, T., Tischkowitz, M., Ameziane, N., Hodgson, S. V., Mathew, C. G., Joenje, H., Mok, S. C., and D'Andrea, A. D. Disruption of the Fanconi anemiaBRCA pathway in cisplatin-sensitive ovarian tumors. Nat Med, 9: 568-574, 2003.

70. Wiltshire, T., Senft, J., Wang, Y., Konat, G. W., Wenger, S. L., Reed, E., and Wang, W. BRCA1 Contributes to Cell Cycle Arrest and Chemoresistance in Response to Anticancer Agent, Irofulven. Mol Pharmacol mol.106.029504, 2007.

71. Tassone, P., Tagliaferri, P., Perricelli, A., Blotta, S., Quaresima, B., Martelli, M., Goel, A., Barbieri, V., Costanzo, F., Boland, C., and Venuta, S. BRCA1 expression modulates chemosensitivity of BRCA1-defective HCC1937 human breast cancer cells. Br J Cancer, 88: 1285-1291, 2003.

72. Quinn, J. E., Kennedy, R. D., Mullan, P. B., Gilmore, P. M., Carty, M., Johnston, P. G., and Harkin, D. P. BRCA1 functions as a differential modulator of chemotherapy-induced apoptosis. Cancer Res, 63: 6221-6228, 2003.

73. Fedier, A., Steiner, R., Schwarz, V., Lenherr, L., Haller, U., and Fink, D. The effect of loss of Brcal on the sensitivity to anticancer agents in p53-deficient cells. Int J Cancer, 22: 1169-1173, 2003.

74. Meza, J. E., Brzovic, P. S., King, M.-C., and Klevit, R. E. Mapping the Functional Domains of BRCA1. INTERACTION OF THE RING FINGER DOMAINS OF BRCA1 AND BARD1. J. Biol. Chem., 274: 5659-5665, 1999. 
75. Wu, L., Wang, Z., Tsan, J., Spillman, M., Phung, A., Xu, X., Yang, M.-C., Hwang, L.-Y., Bowcock, A., and Baer, R. Identification of a RING protein that can interact in vivo with the BRCA1 gene product. Nat Genet, 14: 430-440, 1996.

76. Wang, H., Shao, N., Ming Ding, Q., Cui, J., Reddy, E., and Rao, V. BRCA1 proteins are transported to the nucleus in the absence of serum and splice variants BRCA1a, BRCA1b are tyrosine phosphoproteins that associate with E2F, cyclins and cyclin dependent kinases. Oncogene, 15: 143-157, 1997.

77. Aprelikova, O. N., Fang, B. S., Meissner, E. G., Cotter, S., Campbell, M., Kuthiala, A., Bessho, M., Jensen, R. A., and Liu, E. T. BRCA1-associated growth arrest is RB-dependent. PNAS, 96: 11866-11871, 1999.

78. Wang, Q., Zhang, H., Kajino, K., and Greene, M. BRCA1 binds c-Myc and inhibits its transcriptional and transforming activity in cells. Oncogene, 17: 19391948, 1998.

79. Scully, R., Chen, J., Plug, A., Xiao, Y., Weaver, D., Feunteun, J., Ashley, T., and Livingston, D. M. Association of BRCA1 with Rad51 in mitotic and meiotic cells. Cell, 88: 265-275, 1997.

80. Chaturvedi, P., Eng, W. K., Zhu, Y., Mattern, M. R., Mishra, R., Hurle, M. R., Zhang, X., Annan, R. S., Lu, Q., Faucette, L. F., Scott, G. F., Li, X., Carr, S. A., Johnson, R. K., Winkler, J. D., and Zhou, B. B. Mammalian Chk2 is a downstream effector of the ATM-dependent DNA damage checkpoint pathway. Oncogene, 18: 4047-4054, 1999. 
81. Xu, B., Kim, S., and Kastan, M. B. Involvement of Brca1 in S-phase and G(2)phase checkpoints after ionizing irradiation. Mol Cell Biol, 21: 3445-3450, 2001.

82. Yamane, K., Chen, J., and Kinsella, T. J. Both DNA Topoisomerase II-binding Protein 1 and BRCA1 Regulate the G2-M Cell Cycle Checkpoint. Cancer Res, 63: 3049-3053, 2003.

83. Xu, B., O'Donnell, A. H., Kim, S. T., and Kastan, M. B. Phosphorylation of serine 1387 in Brca1 is specifically required for the Atm-mediated S-phase checkpoint after ionizing irradiation. Cancer Res, 62: 4588-4591, 2002.

84. Ouchi, T., Monteiro, A. N. A., August, A., Aaronson, S. A., and Hanafusa, H. BRCA1 regulates p53-dependent gene expression. PNAS, 95: 2302-2306, 1998.

85. Zhang, H., Somasundaram, K., Peng, Y., Zhang, H., Bi, D., Weber, B., and ElDeiry, W. BRCA1 physically associates with $\mathrm{p} 53$ and stimulates its transcriptional activity. Oncogene, 16: 1713-1721, 1998.

86. Chai, Y., Cui, J., Shao, N., Reddy, E., and Rao, V. The second BRCT domain of BRCA1 proteins interacts with p53 and stimulates transcription from the p21WAF1/CIP1 promoter. Oncogene, 18: 263-268, 1999.

87. Scully, R., Anderson, S. F., Chao, D. M., Wei, W., Ye, L., Young, R. A., Livingston, D. M., and Parvin, J. D. BRCA1 is a component of the RNA polymerase II holoenzyme. Proc Natl Acad Sci U S A, 94: 5605-5610, 1997. 
88. Yarden, R. I. and Brody, L. C. BRCA1 interacts with components of the histone deacetylase complex. PNAS, 96: 4983-4988, 1999.

89. Chen, J., Silver, D. P., Walpita, D., Cantor, S. B., Gazdar, A. F., Tomlinson, G., Couch, F. J., Weber, B. L., Ashley, T., Livingston, D. M., and Scully, R. Stable interaction between the products of the BRCA1 and BRCA2 tumor suppressor genes in mitotic and meiotic cells. Mol Cell, 2: 317-328, 1998.

90. Anderson, S., Schlegel, B., Nakajima, T., Wolpin, E., and Parvin, J. BRCA1 protein is linked to the RNA polymerase II holoenzyme complex via RNA helicase A. Nat Genet, 19: 254-256, 1998.

91. Yu, X., Chini, C. C., He, M., Mer, G., and Chen, J. The BRCT domain is a phospho-protein binding domain. Science, 302: 639-642, 2003.

92. Manke, I. A., Lowery, D. M., Nguyen, A., and Yaffe, M. B. BRCT repeats as phosphopeptide-binding modules involved in protein targeting. Science, 302: 636-639, 2003.

93. Yu, X. and Chen, J. DNA Damage-Induced Cell Cycle Checkpoint Control Requires CtIP, a Phosphorylation-Dependent Binding Partner of BRCA1 CTerminal Domains. Mol. Cell. Biol., 24: 9478-9486, 2004.

94. Tomlinson, G. E., Chen, T. T., Stastny, V. A., Virmani, A. K., Spillman, M. A., Tonk, V., Blum, J. L., Schneider, N. R., Wistuba, II, Shay, J. W., Minna, J. D., and Gazdar, A. F. Characterization of a breast cancer cell line derived from a germ-line BRCA1 mutation carrier. Cancer Res, 58: 3237-3242, 1998. 
95. Scully, R., Ganesan, S., Vlasakova, K., Chen, J., Socolovsky, M., and Livingston, D. M. Genetic analysis of BRCA1 function in a defined tumor cell line. Mol Cell, 4: 1093-1099, 1999.

96. Chen, J. J., Silver, D., Cantor, S., Livingston, D. M., and Scully, R. BRCA1, BRCA2, and Rad51 operate in a common DNA damage response pathway. Cancer Res, 59: 1752s-1756s, 1999.

97. Zhong, Q., Boyer, T. G., Chen, P. L., and Lee, W. H. Deficient nonhomologous end-joining activity in cell-free extracts from Brca1-null fibroblasts. Cancer Res, 62: 3966-3970, 2002.

98. Gowen, L. C., Avrutskaya, A. V., Latour, A. M., Koller, B. H., and Leadon, S. A. BRCA1 required for transcription-coupled repair of oxidative DNA damage. Science, 281: 1009-1012, 1998.

99. Moynahan, M. E., Chiu, J. W., Koller, B. H., and Jasin, M. Brca1 controls homology-directed DNA repair. Mol Cell, 4: 511-518, 1999.

100. Zhong, Q., Chen, C. F., Li, S., Chen, Y., Wang, C. C., Xiao, J., Chen, P. L., Sharp, Z. D., and Lee, W. H. Association of BRCA1 with the hRad50-hMre11p95 complex and the DNA damage response. Science, 285: 747-750, 1999.

101. Wang, Y., Cortez, D., Yazdi, P., Neff, N., Elledge, S. J., and Qin, J. BASC, a super complex of BRCA1-associated proteins involved in the recognition and repair of aberrant DNA structures. Genes Dev, 14: 927-939, 2000. 
102. Chapman, M. S. and Verma, I. M. Transcriptional activation by BRCA1. 382: 678-679, 1996.

103. Monteiro, A. N. A., August, A., and Hanafusa, H. Evidence for a transcriptional activation function of BRCA1 C-terminal region. PNAS, 93: 13595-13599, 1996.

104. Neish, A., Anderson, S., Schlegel, B., Wei, W., and Parvin, J. Factors associated with the mammalian RNA polymerase II holoenzyme. Nucl. Acids Res., 26: 847$853,1998$.

105. Brown, A. L., Lee, C. H., Schwarz, J. K., Mitiku, N., Piwnica-Worms, H., and Chung, J. H. A human Cds1-related kinase that functions downstream of ATM protein in the cellular response to DNA damage. Proc Natl Acad Sci U S A, 96: 3745-3750, 1999.

106. Hirao, A., Kong, Y. Y., Matsuoka, S., Wakeham, A., Ruland, J., Yoshida, H., Liu, D., Elledge, S. J., and Mak, T. W. DNA damage-induced activation of $\mathrm{p} 53$ by the checkpoint kinase Chk2. Science, 287: 1824-1827, 2000.

107. Lee, J. S., Collins, K. M., Brown, A. L., Lee, C. H., and Chung, J. H. hCds1mediated phosphorylation of BRCA1 regulates the DNA damage response. Nature, 404: 201-204, 2000.

108. Matsuoka, S., Huang, M., and Elledge, S. J. Linkage of ATM to cell cycle regulation by the Chk2 protein kinase. Science, 282: 1893-1897, 1998. 
109. Stevens, C., Smith, L., and La Thangue, N. B. Chk2 activates E2F-1 in response to DNA damage. Nat Cell Biol, 5: 401-409, 2003.

110. Melchionna, R., Chen, X. B., Blasina, A., and McGowan, C. H. Threonine 68 is required for radiation-induced phosphorylation and activation of Cds1. Nat Cell Biol, 2: 762-765, 2000.

111. Shieh, S. Y., Ahn, J., Tamai, K., Taya, Y., and Prives, C. The human homologs of checkpoint kinases Chk1 and Cds1 (Chk2) phosphorylate p53 at multiple DNA damage-inducible sites. Genes Dev, 14: 289-300, 2000.

112. Shieh, S. Y., Taya, Y., and Prives, C. DNA damage-inducible phosphorylation of p53 at N-terminal sites including a novel site, Ser20, requires tetramerization. Embo J, 18: 1815-1823, 1999.

113. Unger, T., Juven-Gershon, T., Moallem, E., Berger, M., Vogt Sionov, R., Lozano, G., Oren, M., and Haupt, Y. Critical role for Ser20 of human p53 in the negative regulation of p53 by Mdm2. Embo J, 18: 1805-1814, 1999.

114. Chehab, N. H., Malikzay, A., Appel, M., and Halazonetis, T. D. Chk2/hCds1 functions as a DNA damage checkpoint in G(1) by stabilizing p53. Genes Dev, 14: 278-288, 2000.

115. Chehab, N. H., Malikzay, A., Stavridi, E. S., and Halazonetis, T. D. Phosphorylation of Ser-20 mediates stabilization of human p53 in response to DNA damage. Proc Natl Acad Sci U S A, 96: 13777-13782, 1999. 
116. Ahn, J., Urist, M., and Prives, C. The Chk2 protein kinase. DNA Repair (Amst), 8: 1039-1047, 2004.

117. Kim, S. T., Lim, D. S., Canman, C. E., and Kastan, M. B. Substrate specificities and identification of putative substrates of ATM kinase family members. J Biol Chem, 274: 37538-37543, 1999.

118. Ahn, J. and Prives, C. Checkpoint Kinase 2 (Chk2) Monomers or Dimers Phosphorylate Cdc25C after DNA Damage Regardless of Threonine 68 Phosphorylation. J. Biol. Chem., 277: 48418-48426, 2002.

119. Ahn, J.-Y., Li, X., Davis, H. L., and Canman, C. E. Phosphorylation of Threonine 68 Promotes Oligomerization and Autophosphorylation of the Chk2 Protein Kinase via the Forkhead-associated Domain. J. Biol. Chem., 277: 19389-19395, 2002.

120. Xu, X., Tsvetkov, L. M., and Stern, D. F. Chk2 Activation and PhosphorylationDependent Oligomerization. Mol. Cell. Biol., 22: 4419-4432, 2002.

121. Schwarz, J. K., Lovly, C. M., and Piwnica-Worms, H. Regulation of the Chk2 Protein Kinase by Oligomerization-Mediated cis- and trans-Phosphorylation. Mol Cancer Res, 1: 598-609, 2003.

122. Wu, X. and Chen, J. Autophosphorylation of Chk2 at serine-516 is required for radiation-induced apoptosis. J Biol Chem, 2003. 
123. Lee, C.-H. and Chung, J. H. The hCds1 (Chk2)-FHA Domain Is Essential for a Chain of Phosphorylation Events on hCds1 That Is Induced by Ionizing Radiation. J. Biol. Chem., 276: 30537-30541, 2001.

124. Falck, J., Mailand, N., Syljuasen, R. G., Bartek, J., and Lukas, J. The ATM-Chk2Cdc25A checkpoint pathway guards against radioresistant DNA synthesis. Nature, 410: 842-847, 2001.

125. Blasina, A., de Weyer, I. V., Laus, M. C., Luyten, W. H., Parker, A. E., and McGowan, C. H. A human homologue of the checkpoint kinase Cds1 directly inhibits Cdc25 phosphatase. Curr Biol, 9: 1-10, 1999.

126. Hanahan, D. and Weinberg, R. A. The Hallmarks of Cancer. Cell, 100: 57-70, 2000.

127. Igney, F. H. and Krammer, P. H. Death and anti-death: tumour resistance to apoptosis. Nat Rev Cancer, 2: 277-288, 2002.

128. Chang, H. Y. and Yang, X. Proteases for Cell Suicide: Functions and Regulation of Caspases. Microbiol. Mol. Biol. Rev., 64: 821-846, 2000.

129. Thornberry, N. A. and Lazebnik, Y. Caspases: Enemies Within. Science, 281: 1312-1316, 1998.

130. Nicholson, D. W. and Thornberry, N. A. Caspases: killer proteases. Trends Biochem Sci, 8: 299-306, 1997. 
131. Cohen, G. M. Caspases: the executioners of apoptosis. Biochem. J., 326: 1-16, 1997.

132. Orth, K., Chinnaiyan, A. M., Garg, M., Froelich, C. J., and Dixit, V. M. The CED-3/ICE-like Protease Mch2 Is Activated during Apoptosis and Cleaves the Death Substrate Lamin A. J. Biol. Chem., 271: 16443-16446, 1996.

133. Kothakota, S., Azuma, T., Reinhard, C., Klippel, A., Tang, J., Chu, K., McGarry, T. J., Kirschner, M. W., Koths, K., Kwiatkowski, D. J., and Williams, L. T. Caspase-3-Generated Fragment of Gelsolin: Effector of Morphological Change in Apoptosis. Science, 278: 294-298, 1997.

134. Wen, L.-P., Fahrni, J. A., Troie, S., Guan, J.-L., Orth, K., and Rosen, G. D. Cleavage of Focal Adhesion Kinase by Caspases during Apoptosis. J. Biol. Chem., 272: 26056-26061, 1997.

135. Kaufmann, S., Desnoyers, S., Ottaviano, Y., Davidson, N., and Poirier, G. Specific proteolytic cleavage of poly(ADP-ribose) polymerase: an early marker of chemotherapy-induced apoptosis. Cancer Res, 53: 3976-3985, 1993.

136. Norbury, C. J. and Zhivotovsky, B. DNA damage-induced apoptosis. Oncogene, 23: 2797-2808, 2004.

137. Tinel, A. and Tschopp, J. The PIDDosome, a Protein Complex Implicated in Activation of Caspase-2 in Response to Genotoxic Stress. Science, 304: 843-846, 2004. 
138. Zhivotovsky, B. and Orrenius, S. Caspase-2 function in response to DNA damage. Biochemical and Biophysical Research Communications, 331: 859-867, 2005.

139. Paroni, G., Henderson, C., Schneider, C., and Brancolini, C. Caspase-2-induced apoptosis is dependent on caspase-9, but its processing during UV- or tumor necrosis factor-dependent cell death requires caspase-3. J Biol Chem, 276: 2190721915, 2001.

140. Lassus, P., Opitz-Araya, X., and Lazebnik, Y. Requirement for caspase-2 in stress-induced apoptosis before mitochondrial permeabilization. Science, 297: 1352-1354, 2002.

141. Guo, Y., Srinivasula, S. M., Druilhe, A., Fernandes-Alnemri, T., and Alnemri, E. S. Caspase-2 Induces Apoptosis by Releasing Proapoptotic Proteins from Mitochondria. J. Biol. Chem., 277: 13430-13437, 2002.

142. Read, S. H., Baliga, B. C., Ekert, P. G., Vaux, D. L., and Kumar, S. A novel Apaf-1-independent putative caspase-2 activation complex. J. Cell Biol., 159: 739-745, 2002.

143. Lin, C.-F., Chen, C.-L., Chang, W.-T., Jan, M.-S., Hsu, L.-J., Wu, R.-H., Tang, M.-J., Chang, W.-C., and Lin, Y.-S. Sequential Caspase-2 and Caspase-8 Activation Upstream of Mitochondria during Ceramideand Etoposide-induced Apoptosis. J. Biol. Chem., 279: 40755-40761, 2004.

144. Ashkenazi, A. and Dixit, V. M. Death receptors: signaling and modulation. Science, 281: 1305-1308, 1998. 
145. Boldin, M. P., Goncharov, T. M., Goltsev, Y. V., and Wallach, D. Involvement of MACH, a novel MORT1/FADD-interacting protease, in Fas/APO-1- and TNF receptor-induced cell death. Cell, 85: 803-815, 1996.

146. Chinnaiyan, A. M., O'Rourke, K., Tewari, M., and Dixit, V. FADD, a novel death domain-containing protein, interacts with the death domain of Fas and initiates apoptosis. Cell, 81: 505-512, 1995.

147. Muzio, M., Chinnaiyan, A. M., Kischkel, F. C., O'Rourke, K., Shevchenko, A., Ni, J., Scaffidi, C., Bretz, J. D., Zhang, M., Gentz, R., Mann, M., Krammer, P. H., Peter, M. E., and Dixit, V. FLICE, a novel FADD-homologous ICE/CED-3-like protease, is recruited to the CD95 (Fas/APO-1) death--inducing signaling complex. Cell, 85: 817-827, 1996.

148. Kischkel, F. C., Hellbardt, S., Behrmann, I., Germer, M., Pawlita, M., Krammer, P. H., and Peter, M. E. Cytotoxicity-dependent APO-1 (Fas/CD95)-associated proteins form a death-inducing signaling complex (DISC) with the receptor. EMBO Journal, 14: 5579-5588, 1995.

149. Yang, X., Chang, H. Y., and Baltimore, D. Autoproteolytic Activation of ProCaspases by Oligomerization. Molecular Cell, 1: 319-325, 1998.

150. Muzio, M., Stockwell, B. R., Stennicke, H. R., Salvesen, G. S., and Dixit, V. M. An Induced Proximity Model for Caspase-8 Activation. J. Biol. Chem., 273: 2926-2930, 1998. 
151. Shin, S., Lee, Y., Kim, W., Ko, H., Choi, H., and Kim, K. Caspase-2 primes cancer cells for TRAIL-mediated apoptosis by processing procaspase- 8 . The EMBO Journal, 24: 3532-3542, 2005.

152. Li, H., Zhu, H., Xu, C. J., and Yuan, J. Cleavage of BID by caspase 8 mediates the mitochondrial damage in the Fas pathway of apoptosis. Cell, 94: 491-501, 1998.

153. Srinivasula, S. M., Ahmad, M., Fernandes-Alnemri, T., and Alnemri, E. S. Autoactivation of procaspase-9 by Apaf-1-mediated oligomerization. Mol Cell, 1: 949-957, 1998.

154. Zou, H., Li, Y., Liu, X., and Wang, X. An APAF-1·Cytochrome c Multimeric Complex Is a Functional Apoptosome That Activates Procaspase-9. J. Biol. Chem., 274: 11549-11556, 1999.

155. Li, P., Nijhawan, D., Budihardjo, I., Srinivasula, S. M., Ahmad, M., Alnemri, E. S., and Wang, X. Cytochrome $\mathrm{c}$ and dATP-dependent formation of Apaf1/caspase-9 complex initiates an apoptotic protease cascade. Cell, 91: 479-489, 1997.

156. Kanai, M., Tong, W.-M., Sugihara, E., Wang, Z.-Q., Fukasawa, K., and Miwa, M. Involvement of Poly(ADP-Ribose) Polymerase 1 and Poly(ADP-Ribosyl)ation in Regulation of Centrosome Function. Mol. Cell. Biol., 23: 2451-2462, 2003.

157. Kang, T.-B., Ben-Moshe, T., Varfolomeev, E. E., Pewzner-Jung, Y., Yogev, N., Jurewicz, A., Waisman, A., Brenner, O., Haffner, R., Gustafsson, E., 
Ramakrishnan, P., Lapidot, T., and Wallach, D. Caspase-8 Serves Both Apoptotic and Nonapoptotic Roles. J Immunol, 173: 2976-2984, 2004.

158. Helfer, B., Boswell, B. C., Finlay, D., Cipres, A., Vuori, K., Bong Kang, T., Wallach, D., Dorfleutner, A., Lahti, J. M., Flynn, D. C., and Frisch, S. M. Caspase-8 Promotes Cell Motility and Calpain Activity under Nonapoptotic Conditions. Cancer Res, 66: 4273-4278, 2006.

159. Kennedy, N. J., Kataoka, T., Tschopp, J., and Budd, R. C. Caspase Activation Is Required for T Cell Proliferation. J. Exp. Med., 190: 1891-1896, 1999.

160. Boissonnas, A., Bonduelle, O., Lucas, B., Debré, P., Autran, B., and Combadière, B. Differential requirement of caspases during naive T cell proliferation. European Journal of Immunology, 32: 3007-3015, 2002.

161. Sordet, O., Rebe, C., Plenchette, S., Zermati, Y., Hermine, O., Vainchenker, W., Garrido, C., Solary, E., and Dubrez-Daloz, L. Specific involvement of caspases in the differentiation of monocytes into macrophages. Blood, 100: 4446-4453, 2002.

162. De Maria, R., Zeuner, A., Eramo, A., Domenichelli, C., Bonci, D., Grignani, F., Srinivasula, S. M., Alnemri, E. S., Testa, U., and Peschle, C. Negative regulation of erythropoiesis by caspase-mediated cleavage of GATA-1. 401: 489-493, 1999.

163. Zermati, Y., Garrido, C., Amsellem, S., Fishelson, S., Bouscary, D., Valensi, F., Varet, B., Solary, E., and Hermine, O. Caspase Activation Is Required for Terminal Erythroid Differentiation. J. Exp. Med., 193: 247-254, 2001. 
164. Ishizaki, Y., Jacobson, M. D., and Raff, M. C. A Role for Caspases in Lens Fiber Differentiation. J. Cell Biol., 140: 153-158, 1998.

165. de Botton, S., Sabri, S., Daugas, E., Zermati, Y., Guidotti, J. E., Hermine, O., Kroemer, G., Vainchenker, W., and Debili, N. Platelet formation is the consequence of caspase activation within megakaryocytes. Blood, 100: 13101317, 2002.

166. Hidalgo, M., Izbicka, E., Eckhardt, S. G., MacDonald, J. R., Cerna, C., Gomez, L., Rowinsky, E. K., Weitman, S. D., and Von Hoff, D. D. Antitumor activity of MGI 114 (6-hydroxymethylacylfulvene, HMAF), a semisynthetic derivative of illudin S, against adult and pediatric human tumor colony-forming units. Anticancer Drugs, 10: 837-844, 1999.

167. MacDonald, J. R., Muscoplat, C. C., Dexter, D. L., Mangold, G. L., Chen, S. F., Kelner, M. J., McMorris, T. C., and Von Hoff, D. D. Preclinical antitumor activity of 6-hydroxymethylacylfulvene, a semisynthetic derivative of the mushroom toxin illudin S. Cancer Res, 57: 279-283, 1997.

168. Sato, Y., Kashimoto, S., MacDonald, J. R., and Nakano, K. In vivo antitumour efficacy of MGI-114 (6-hydroxymethylacylfulvene, HMAF) in various human tumour xenograft models including several lung and gastric tumours. Eur J Cancer, 37: 1419-1428, 2001. 
169. Senzer, N., Arsenau, J., Richards, D., Berman, B., MacDonald, J. R., and Smith, S. Irofulven demonstrates clinical activity against metastatic hormone-refractory prostate cancer in a phase 2 single-agent trial. Am J Clin Oncol, 28: 36-42, 2005.

170. Thomas, J. P., Arzoomanian, R., Alberti, D., Feierabend, C., Binger, K., Tutsch, K. D., Steele, T., Marnocha, R., Smith, C., Smith, S., MacDonald, J., Wilding, G., and Bailey, H. Phase I clinical and pharmacokinetic trial of irofulven. Cancer Chemother Pharmacol, 48: 467-472, 2001.

171. Giles, F., Cortes, J., Garcia-Manero, G., Kornblau, S., Estey, E., Kwari, M., Murgo, A., and Kantarjian, H. Phase I study of irofulven (MGI 114), an acylfulvene illudin analog, in patients with acute leukemia. Invest New Drugs, 19: 13-20, 2001.

172. Murgo, A., Cannon, D. J., Blatner, G., and Cheson, B. D. Clinical trials referral resource. Clinical trials of MGI-114. Oncology (Huntingt), 13: 233, 237-238, 1999.

173. Jaspers, N. G., Raams, A., Kelner, M. J., Ng, J. M., Yamashita, Y. M., Takeda, S., McMorris, T. C., and Hoeijmakers, J. H. Anti-tumour compounds illudin S and Irofulven induce DNA lesions ignored by global repair and exclusively processed by transcription- and replication-coupled repair pathways. DNA Repair (Amst), 1: 1027-1038, 2002.

174. Wang, J., Wiltshire, T., Wang, Y., Mikell, C., Burks, J., Cunningham, C., Van Laar, E. S., Waters, S. J., Reed, E., and Wang, W. ATM-dependent CHK2 
Activation Induced by Anticancer Agent, Irofulven. J Biol Chem, 279: 3958439592, 2004.

175. Wang, Y., Wiltshire, T., Senft, J., Reed, E., and Wang, W. Irofulven induces replication-dependent CHK2 activation related to p53 status. Biochem Pharmacol, 73: 469-480, 2007.

176. Wang, Y., Wiltshire, T., Senft, J., Wenger, S. L., Reed, E., and Wang, W. Fanconi anemia D2 protein confers chemoresistance in response to the anticancer agent, irofulven. Mol Cancer Ther, 5: 3153-3161, 2006.

177. Wang, W., Waters, S. J., MacDonald, J. R., Roth, C., Shentu, S., Freeman, J., Von Hoff, D. D., and Miller, A. R. Irofulven (6-hydroxymethylacylfulvene, MGI 114)induced apoptosis in human pancreatic cancer cells is mediated by ERK and JNK kinases. Anticancer Res, 22: 559-564, 2002.

178. Wang, W., Waters, S. J., MacDonald, J. R., Von Hoff, D. D., Strodel, W. E., and Miller, A. R. Irofulven (6-hydroxymethylacylfulvene, MGI 114) induces caspase 8 and 9-mediated apoptosis in human pancreatic adenocarcinoma cells. Anticancer Res, 21: 1789-1794, 2001.

179. Liang, H., Salinas, R. A., Leal, B. Z., Kosakowska-Cholody, T., Michejda, C. J., Waters, S. J., Herman, T. S., Woynarowski, J. M., and Woynarowska, B. A. Caspase-mediated apoptosis and caspase-independent cell death induced by irofulven in prostate cancer cells. Mol Cancer Ther, 3: 1385-1396, 2004. 


\section{Chapter II}

\section{BRCA1 Contributes to Cell Cycle Arrest and Chemoresistance in Response to Anticancer Agent, Irofulven}

Timothy Wiltshire, Jamie Senft, Yutian Wang, Gregory W. Konat, Sharon L. Wenger, Eddie Reed, ${ }^{1}$ and Weixin Wang

Department of Microbiology, Immunology and Cell Biology (T.W., E.R., W.W.); Mary Babb Randolph Cancer Center (J.S., Y.W., E.R., W.W.); Department of Neurobiology and Anatomy (G.W.K.); and Department of Pathology (S.L.W.); West Virginia University School of Medicine, Morgantown, WV 26506.

Abbreviations: IR, ionizing radiation; HR, homologous recombination; DSBs, DNA double-strand breaks; sh-RNA, short-hairpin RNA; RDS, radioresistant DNA synthesis assay; PFGE, pulse-field gel electrophoresis; FISH, fluorescent in situ hybridization.

This manuscript was published in Molecular Pharmacology, Vol 71, Issue 4 (2007) 10511060 . 


\section{ABSTRACT}

Tumor suppressor gene BRCA1 is frequently mutated in familial breast and ovarian cancer. BRCA1 plays pivotal roles in maintaining genomic stability by interacting with numerous proteins in cell cycle control and DNA repair. Irofulven (6hydroxymethylacylfulvene, HMAF, MGI 114, NSC\#: 683863) is one of a new class of anticancer agents that are analogs of mushroom-derived illudin toxins. Preclinical studies and clinical trials have demonstrated that irofulven is effective against several tumor cell types. The exact nature of irofulven-induced DNA damage is not completely understood. Previously, we have demonstrated that irofulven activates ATM and its targets, NBS1, SMC1, CHK2 and p53. In this study, we hypothesize that irofulven induces DNA doublestrand breaks and that BRCA1 may affect chemosensitivity by controlling cell cycle checkpoints, DNA repair and genomic stability in response to irofulven treatment. We observed that irofulven induces the formation of chromosome breaks and radials as well as the activation and foci formation of $\gamma$-H2AX, BRCA1 and RAD51. We also provided evidence that irofulven induces the generation of DNA double-strand breaks. By using BRCA1-deficient or proficient cells, we demonstrated that in response to irofulven, BRCA1 contributes to the control of $\mathrm{S}$ and $\mathrm{G} 2 / \mathrm{M}$ cell cycle arrest, is critical for repairing DNA double-strand breaks and for RAD51-dependent homologous recombination. Furthermore, we found that BRCA1 deficiency results in increased chromosome damage and chemosensitivity after irofulven treatment. 


\section{INTRODUCTION}

Tumor suppressor BRCA1 is frequently mutated in familial breast and ovarian cancer $(1,2)$. More than ten percent of women with breast or ovarian cancer carry BRCA1 mutations $(2,3)$. BRCA1 is involved in multiple cellular processes including cell cycle checkpoint control, chromosome remodeling, transcriptional regulation, DNA repair and apoptosis $(1,2,4)$. It is required for both $\mathrm{S}$ and $\mathrm{G} 2 / \mathrm{M}$ checkpoints in response to ionizing radiation (IR). Moreover, it plays important roles in multiple DNA repair pathways including homologous recombination (HR) and transcription-coupled nucleotide excision repair (TC-NER) $(1,2,5)$. In response to DNA double-strand breaks (DSBs), proteins such as H2AX, RAD51, MRE11, RAD50, NBS1 and BRCA1 are rapidly phosphorylated by ATM and/or ATR kinases and form foci at the damaged sites. BRCA1 interacts with many of these DNA damage signaling and DNA repair proteins including $\gamma$-H2AX and RAD51 $(1,2,4,6)$. The $\gamma$-H2AX foci formation functions to recruit DNA repair factors to the damaged sites, enforcing HR repair of DNA DSBs and linking chromatin remodeling to DNA repair (7-10). RAD51 is a DNA recombinase and an essential protein in initiating the HR process by mediating DNA strand exchange during recombination. BRCA1 is required for RAD51 foci assembly in response to IRinduced DNA DSBs $(1,2,6)$.

Irofulven (6-hydroxymethylacylfulvene, HMAF, MGI 114, NSC\#: 683863) is one of a new class of anticancer agents that are analogs of mushroom-derived illudin toxins. Preclinical studies and clinical trials have demonstrated that irofulven is effective against several tumor cell types (11-19). Earlier studies have suggested that the DNA damage 
caused by the illudin family of compounds might be repaired by the nucleotide excision repair (NER) pathway $(20,21)$. Recent studies suggested that TC-NER was the exclusive repair pathway in repairing illudin S and irofulven-elicited DNA lesions and that irofulven cytotoxicity was influenced by the expression of excision endonuclease XPG $(22,23)$. However, the HR pathway for DSB repair was not evaluated in these studies (20-23), even though it was suggested as a potential mechanism likely affecting sensitivity to irofulven (22). Nonetheless, the structure and nature of DNA damage caused by irofulven have not been characterized. Recent reports indicated that ATM and CHK2 were specifically activated by IR or drug (calicheamicin)-induced DSBs (24-28). Previously, we have demonstrated that irofulven activates ATM and its targets, NBS1, SMC1, CHK2 and p53 (29). Based on these findings, we hypothesize that irofulven induces DNA DSBs and, as a result, BRCA1 may confer chemoresistance to irofulven by controlling cell cycle checkpoints, DNA repair and genomic stability. Therefore, BRCA1 deficiency might be a useful target and predictive marker for chemotherapy by irofulven.

To further understand the mechanisms of action involved with irofulven, we investigated the role that BRCA1 might play in irofulven-induced DNA damage response. We have observed that irofulven induces the formation of chromosomal breaks and radials as well as the activation and foci formation of $\gamma$-H2AX, BRCA1 and RAD51. We have provided evidence that irofulven induces the generation of DSBs. Furthermore, we have demonstrated that in response to irofulven, BRCA1 controls S and G2/M checkpoints and is critical for repairing DNA double-strand breaks through RAD51dependent homologous recombination, and BRCA1 deficiency results in increased chromosome damage and chemosensitivity. 


\section{MATERIALS AND METHODS}

Cell culture. All cell lines were maintained in various media supplemented with $10 \%$ fetal bovine serum in a $37^{\circ} \mathrm{C}$ incubator with $5 \% \mathrm{CO}_{2}$ atmosphere. Ovarian cancer cell lines A2780, CAOV3 and OVCAR3 were cultured in RPMI 1640; SKOV3 was cultured in McCoy's 5A medium. The vector and BRCA1-transfected breast cancer cell line HCC1937 cells (generously provided by Professor Ralph Scully of Dana-Farber Cancer Institute, Harvard Medical School, Boston, MA) were cultured in ACL4 medium as described (30). The vector and short-hairpin BRCA1 (sh-BRCA1) stably transfected SKOV3 cells were cultured in McCoy's 5A medium containing $200 \mu \mathrm{g} / \mathrm{ml}$ of G418 (Invitrogen, Carlsbad, CA).

Clonogenic survival assay. To determine chemosensitivity and 1xIC50 concentration, clonogenic survival assay was performed as described previously (29) on 60-mm cell culture dishes. Cells were treated with different concentrations of irofulven for one hour followed by drug-free incubations for about 10 days. Colonies were stained with crystal violet and colonies with 50 or more cells were counted.

Metaphase spread. Cells were treated with irofulven. Colcemid (400 ng/ml) (Calbiochem, La Jolla, CA) was added to medium four hours before harvesting. After trypsinization, cells were washed once with PBS. Cell pellets were re-suspended in 75 $\mathrm{mM} \mathrm{KCl}$ and placed in a $37^{\circ} \mathrm{C}$ incubator for eight minutes. After centrifugation, cells were fixed for two hours at $4^{\circ} \mathrm{C}$ using 3:1 absolute methanol to glacial acetic acid and 
then washed twice with fixative. Cells were re-suspended in fixative and dropped onto slides. Slides were air-dried at room temperature and stained with 5\% Gurr's Giemsa stain (Biomedical Specialties, Santa Monica, CA) for seven minutes. Slides were rinsed twice with distilled water and air-dried. The images were recorded by an Olympus Provis AX70 light/fluorescence microscope (Olympus, Melville, NY) and Spot digital camera and software (Diagnostic Instruments, Sterling Height, MI).

Western blotting. Western blot was performed as described previously (29). Antibodies used were: monoclonal anti-actin (Sigma, St Louis, MO) and monoclonal anti-BRCA1 (Calbiochem, La Jolla, CA).

Immunofluorescent staining and confocal microscopy. Cells were plated on coverslips and treated with 1xIC50 concentration of irofulven for one hour followed by 12 hours of drug-free incubation. Cells were then fixed and stained with polyclonal antibody against BRCA1 or RAD51 (Santa Cruz Biotechnology, Santa Cruz, CA) and monoclonal antibody against $\gamma$-H2AX (Upstate, Charlottesville, VA). After staining with Alexafluor 488-conjugated goat anti-mouse or goat anti-rabbit; or Alexafluor 546-conjugated goat anti-rabbit secondary antibodies (Invitrogen, Carlsbad, CA), slides were mounted with Vectashield mounting medium (Vector Laboratories, Burlingame, CA) containing 5 $\mathrm{ng} / \mathrm{ml}$ of DAPI. Single color staining images were captured by an Olympus Provis AX70 fluorescence microscope (Olympus, Melville, NY) and Spot digital camera and software (Diagnostic Instruments, Sterling Height, MI). Confocal staining images were captured by a Zeiss LSM510 confocal microscope (Zeiss, Thornwood, NY). 
Radioresistant DNA synthesis (RDS) assay. The RDS assay was performed as described (31). Briefly, cells in the logarithmic phase of growth were pre-labeled by culturing in medium containing $10 \mathrm{nCi}$ of $\left[{ }^{14} \mathrm{C}\right]$-thymidine (PerkinElmer, Boston, MA) for 24 hours. The medium was then replaced with normal medium, and cells were incubated for another 24 hours. Cells were treated with irofulven for one hour and incubated in drug-free medium for 12 hours. Cells were then pulse-labeled with $2.5 \mu \mathrm{Ci}$ of $\left[{ }^{3} \mathrm{H}\right]$-thymidine (PerkinElmer, Boston, MA) for 15 minutes. Cells were harvested, washed twice with PBS, and fixed in 70\% methanol for at least 30 minutes. Cells were then transferred to Whatman filters (Whatman, Clifton, NJ) and washed sequentially with $70 \%$ and then $95 \%$ methanol. The filters were air-dried and the amount of radioactivity was quantified in Wallac 1410 liquid scintillation counter (PerkinElmer, Downers Grove, IL). The resulting ratio of ${ }^{3} \mathrm{H}$ counts per minute to ${ }^{14} \mathrm{C}$ counts per minute, corrected for those counts per minute that were the results of channel crossover, was a measure of DNA synthesis.

Phosphorylated histone H3 staining and flow cytometry. The phospho-histone H3 staining was performed as described (31). Briefly, the vector and BRCA1-transfected HCC1937 cells were treated with $1 \mu \mathrm{M}$ of irofulven for one hour followed by one hour of drug-free incubation. Cells were harvested and fixed in 70\% ethanol. The fixed cells were washed twice with PBS and made permeable with $0.25 \%$ Triton X-100 in PBS on ice for 10 minutes. Cells were rinsed in 1\% BSA/PBS and then stained with rabbit anti-phosphoS10 histone H3 antibody (Upstate, Charlottesville, VA) for two hours at room 
temperature. Cells were rinsed in 1\% BSA/PBS and stained with Alexafluor 488conjugated anti-rabbit secondary antibody for 30 minutes at room temperature. Cells were washed twice with PBS and suspended in PBS containing propidium iodide $(0.25$ $\mu \mathrm{g} / \mathrm{ml})$ and RNase A $(20 \mu \mathrm{g} / \mathrm{ml})$. Flow cytometry was performed on FACSCalibur with CellquestPro software (Becton Dickinson, San Jose, CA). Thirty thousands events were recorded for each sample. The percentage of mitotic cells was determined as those cells that were Alexafluor-positive and contained 4N DNA content.

Mitotic index. Cells were plated on cover-slips and treated with 1xIC50 concentration of irofulven for one hour followed by 24 hours of drug-free incubation. Cells were then fixed and stained with DAPI (5 ng/ml). Staining images were captured by an Olympus Provis AX70 fluorescence microscope (Olympus, Melville, NY) and Spot digital camera and software (Diagnostic Instruments, Sterling Height, MI). In each group, about 4,000 cells were counted. Mitotic index was calculated as the percentage of cells in mitosis.

Pulse-field gel electrophoresis (PFGE). The PFGE was conducted as follows. Cells were scraped from the dish and washed with ice-cold embedding buffer (15 mM Tris$\mathrm{HCl}, \mathrm{pH}$ 7.4, 1mM EGTA, 2mM EDTA, $60 \mathrm{mM} \mathrm{KCl,} \mathrm{15mM} \mathrm{NaCl,} \mathrm{0.5mM} \mathrm{spermidine}$ and $0.15 \mathrm{mM}$ spermine). Cells were then re-suspended in embedding buffer, mixed well, and incubated for 30 seconds in a $30^{\circ} \mathrm{C}$ water bath before adding $1.6 \%$ low melting point agarose pre-warmed to $55^{\circ} \mathrm{C}$. After thorough mixing, the cell suspension was aspirated into $2.3 \mathrm{~mm}$ inner diameter tubing using a syringe. The tube was immediately placed in ice-cold water for 5 minutes to allow the agarose to harden. The agarose core was 
incubated in extraction buffer (10mM Tris-HCl, $\mathrm{pH} 9.5,10 \mathrm{mM} \mathrm{NaCl}, 25 \mathrm{mM}$ EDTA, $1.5 \%$ SDS and $0.1 \%$ mercaptoethanol) overnight at room temperature with gentle agitation. Extraction was performed three more times for two hours each followed by three washes of two hours in TE buffer. The agarose core was then cut into $6 \mathrm{~mm}-\mathrm{long}$ plugs. A 1\% agarose gel (PFGE-certified, Bio-Rad, Hercules, CA) in 0.5X TBE (Cambrex, Rockland, ME) was cast and plugs were inserted into gel wells. The concatenated chromosomes of lambda phage ( $48.5 \mathrm{~kb}$ ) (Bio-Rad, Hercules, CA) were used as the standard for DNA size. The DNA was resolved by direct current of $100 \mathrm{~V}$ for 20 minutes followed by 17 hours of pulse current using a programmable power inverter PPI-200 (MJ Research, Watertown, MA) and program number 6. DNA was visualized by staining with $0.5 \mu \mathrm{g} / \mathrm{ml}$ of ethidium bromide (Invitrogen, Carlsbad, CA) and pictures were captured using Eagle Eye II system and software (Stratagene, La Jolla, CA).

Comet Assay. The comet assay (Trevigen Inc., Gaithersburg, MD) was performed according to manufacturer's protocol by using neutral conditions to mainly detect doublestrand breaks. Briefly, cells were harvested, washed with ice cold PBS, and combined with molten LMAgarose, and $75 \mu \mathrm{l}$ (500-1000 cells) was immediately added to Comet Slide. After hardening, slides were incubated for 30 minutes in lysis solution at $4^{\circ} \mathrm{C}$, then rinsed with $1 \mathrm{X}$ TBE before electrophoresis for 60 minutes at 30V. Slides were rinsed with $\mathrm{dH}_{2} \mathrm{O}$ and placed in $70 \%$ ethanol for 10 minutes, then air-dried. To visualize DNA, $50 \mu 1$ of a 1:1000 dilution of SYBR Green (Invitrogen, Carlsbad, CA) in PBS was added to each slide. Slides were visually scored using Olympus Provis AX70 microscope (Olympus, Melville, NY) from 0-4 based on tail length and intensity, and a total score of 
75 cells was used to determine relative amount of double-strand breaks for each time point. Images were captured using Spot digital camera and software (Diagnostic Instruments, Sterling Height, MI).

Fluorescent in situ hybridization (FISH). Slides were rinsed at room temperature in 2xSSC solution ( $0.3 \mathrm{M}$ sodium chloride and $0.03 \mathrm{M}$ sodium citrate, $\mathrm{pH}$ 7) for 30 minutes and then rinsed in PBS for 15 minutes. Slides were then fixed in 3.7\% formaldehyde/PBS solution for 15 minutes, followed by a $5 \%$ pepsin/ $/ 0.01 \mathrm{M} \mathrm{HCl}$ solution at $37^{\circ} \mathrm{C}$ for 15 minutes. Slides were washed in PBS for 5 minutes at room temperature. Slides were put into 95\% ethanol for 5 minutes and then air-dried. For FISH hybridization, the Whole Chromosome 1 Probe (Oncor, Gaithersburg, Maryland) was pre-warmed to $37^{\circ} \mathrm{C}$ for 5 minutes. Aliquots of $3 \mu \mathrm{l}$ of probe were applied to the slides, covered with $12 \mathrm{~mm}$ diameter round cover-slips and sealed. The slides were then co-denatured at $74^{\circ} \mathrm{C}$ for 6 minutes and placed in a $37^{\circ} \mathrm{C}$ water bath overnight. Slides were washed according to manufacturer's protocol and detected using the Rhodamine-labeled anti-Digoxigenin detection reagent (Insitus, Albuquerque, New Mexico). Slides were then counterstained with DAPI and evaluated using an AxioPlan II epi-fluorescence microscope (Zeiss, Thornwood, NY) and CytoVision software (Applied Imaging, San Jose, California).

RNA interference. Three pairs of 65-nt short-hairpin BRCA1 (sh-BRCA1) oligos containing target sequences of AACCTGTCTCCACAAAGTGTG, AAAGTACGAGATTTAGTCAAC and AAGCAGCGGATACAACCTCAA were designed and synthesized. After annealing, these three 65-bp double-strand sh-BRCA1 
fragments were inserted into pSilencer 2.1-U6-neo vector (Ambion, Austin, TX) and transfected into SKOV3 cells. The pSilencer 2.1-U6-neo vector containing the scrambled sequence was transfected as the non-specific control. Stable cell lines were established by selecting in medium containing G418. 


\section{RESULTS}

\section{Irofulven induces chromosome aberrations and activates BRCA1.}

To characterize the DNA damage caused by irofulven and to examine whether DNA DSBs were generated after treatment, mitotic spread experiments were performed in breast cancer cell line HCC1937 and ovarian cancer cell line SKOV3. HCC1937 cell line is known to express a truncated BRCA1 protein $(30,32)$. SKOV3 cell line is known to harbor a functional BRCA1 (33). Cell lines were treated with irofulven for one hour followed by 24 hours of drug-free incubation. The mitotic spread results clearly demonstrated the induction of chromosome breaks and radials in these cells (Fig. 1A). Similar chromosome breaks and radials were also observed in ovarian cancer cell lines A2780, CAOV3 and OVCAR3 after treatment (data not shown). These results demonstrate that irofulven indeed induces the generation of DNA DSBs.

Upon the induction of DSBs, histone variant $\mathrm{H} 2 \mathrm{AX}$ is rapidly phosphorylated $(\gamma-$ $\mathrm{H} 2 \mathrm{AX}$ ) and forms discrete nuclear foci co-localizing with many other DNA repair proteins such as RAD50, RAD51 and $\operatorname{BRCA} 1(34,35)$. The $\gamma$-H2AX foci formation also allows a sensitive detection of DSBs (34-40). To further confirm that irofulven induces the generation of DSBs, SKOV3 cells were treated with irofulven and immunofluorescently stained utilizing antibodies that recognize $\gamma$-H2AX and BRCA1. Confocal microscopic images indicated that $\gamma$-H2AX and BRCA1 form co-localizing foci after treatment (Fig. 1B). Taken together, these findings demonstrate that irofulven induces the generation of DNA DSBs, which results in the activation and foci formation of $\gamma-\mathrm{H} 2 \mathrm{AX}$ and BRCA1. 


\section{BRCA1 contributes to the control of $S$ and G2/M checkpoints in response to irofulven-induced DNA damage.}

To explore the possible role that BRCA1 activation might play in regulating cell cycle progression after irofulven treatment, we first characterized the cell cycle arrest at S phase by the radioresistant DNA synthesis (RDS) assay. The vector and BRCA1transfected HCC1937 cells were treated with increasing concentrations of irofulven for one hour followed by 12 hours of drug-free incubation. The results of the RDS assay demonstrated that DNA synthesis was significantly inhibited in the BRCA1-transfected HCC1937 cells compared with the vector-transfected cells (Fig. 2A). This indicates that BRCA1 does contribute to the control of $\mathrm{S}$ phase cell cycle arrest in response to irofulven.

It has been reported that there are two distinct G2/M checkpoints in response to IR-induced DSBs, which control the transient G2/M transition and prolonged G2/M accumulation, respectively. BRCA1 is involved in controlling both G2/M checkpoints (31). To study the role that BRCA1 plays in modulating the G2/M checkpoints, immunofluorescent staining for phospho-histone $\mathrm{H} 3$, a marker for mitosis, and FACS analysis were performed to assess the transient $\mathrm{G} 2 / \mathrm{M}$ checkpoint. The vector and BRCA1-transfected HCC1937 cells were treated with irofulven for one hour followed by one hour of drug-free incubation. The FACS analysis results indicated that the phosphohistone $\mathrm{H} 3$-positive population was increased from $1.14 \%$ to $1.63 \%$ in vector-transfected cells; while in BRCA1-transfected cells it was dramatically decreased from $1.25 \%$ to 
$0.55 \%$ (Fig. 2B). These results indicate that BRCA1 controls the G2/M checkpoint in response to irofulven treatment.

The cumulative effect of BRCA1 on S and G2/M checkpoints was also reflected by assessing the mitotic index. The vector and BRCA1-transfected HCC1937 cells were treated with irofulven for one hour followed by 24 hours of drug-free incubation. The mitotic index decreased only $19 \%$ (from $2.7 \%$ to $2.18 \%$ ) in vector-transfected HCC1937 cells; while it dropped 90\% (from 1.56\% to 0.15\%) in BRCA1-transfected HCC1937 cells after treatment (Fig. 2C). Taken together, these results demonstrate that BRCA1 contributes to both $\mathrm{S}$ and $\mathrm{G} 2 / \mathrm{M}$ checkpoints in response to irofulven-induced DNA damage.

\section{BRCA1 is critical for repairing irofulven-induced DSBs and for RAD51-mediated homologous recombination.}

From the data described above, it was demonstrated that irofulven induces chromosome aberrations (breaks and radials) and the foci formation of $\gamma-\mathrm{H} 2 \mathrm{AX}$ and BRCA1. These findings indicate that irofulven induces the generation of DSBs and activation of BRCA1. Therefore, we hypothesize that BRCA1 might play a critical role in regulating the repair of irofulven-induced DSBs. To examine this hypothesis, the BRCA1-proficient and deficient HCC1937 cell lines were again used to compare the DNA repair dynamics and to assess the foci formation of DNA repair factors.

We first compare the differences in the occurrence and repair of irofulveninduced DSBs by pulse-field gel electrophoresis (PFGE). As shown in Fig. 3A, after onehour of treatment, the large genomic DNA fragments from 50 to $>400 \mathrm{~kb}$ gradually 
increased in vector-transfected HCC1937 cells over the period of 6-48 hours. In BRCA1transfected HCC1937 cells, these DSBs were significantly repaired under the same treatment conditions (Fig. 3A).

To confirm these results, we performed the Comet assay under neutral electrophoresis conditions that will predominantly detect DSBs. The results again indicated that irofulven-induced DSBs were significantly repaired in BRCA1-transfected HCC1937 cells 48 or 72 hours after treatment $(p<0.05)$ or $p<0.01$, respectively) (Fig. 3, B and C).

To further examine the differences in repair dynamics of irofulven-induced DSBs, we stained cells for $\gamma-\mathrm{H} 2 \mathrm{AX}$ foci formation over the time period of 72 hours after treatment. The immunofluorescent staining results indicated that the percentage of cells containing $\gamma-\mathrm{H} 2 \mathrm{AX}$ foci started decreasing dramatically in BRCA1-transfected HCC1937 cells 24 hours after treatment (Fig. 3D). Taken together, these results demonstrate that BRCA1 plays an important role in repairing irofulven-induced DSBs.

RAD51 is a DNA recombinase and an essential protein for initiating the strand invasion process in the HR repair of DNA DSBs $(1,2)$. RAD51 forms foci in response to IR-induced DNA DSBs and BRCA1 is required for RAD51 foci formation $(6,41)$. We therefore examined the RAD51 foci formation in the vector and BRCA1-transfected HCC1937 cells. Immunofluorescent staining results demonstrated that, upon irofulven treatment, more RAD51 foci were assembled in BRCA1-transfected HCC1937 cells (from $4.7 \%$ to $40.3 \%$ ) than in vector-transfected $\mathrm{HCC} 1937$ cells (from $2.8 \%$ to $13.2 \%$ ) (Fig. 3, E and F). When the $\gamma$-H2AX foci formation was evaluated under the same conditions, it was observed that $\gamma-\mathrm{H} 2 \mathrm{AX}$ assembled foci to the similar extent in both cells 
(Fig. 3, E and F). These results demonstrate that a similar amount of DSBs were induced by irofulven and RAD51 foci formation is dependent on BRCA1. Taken together, these results demonstrate that BRCA1 plays an important role in repairing irofulven-induced DSBs, RAD51-dependent HR repair is involved and BRCA1 is critical for this process.

\section{BRCA1 contributes to maintaining chromosome integrity upon exposure to irofulven.}

Since BRCA1 controls $\mathrm{S}$ and G2/M cell cycle arrest and is important in repairing irofulven-induced DSBs, we hypothesize that BRCA1 contributes to maintaining chromosome integrity upon exposure to irofulven. Mitotic spread experiments were again performed in the vector and BRCA1-transfected HCC1937 cells. A dramatic increase in chromosome breaks and radials were observed in vector-transfected cells compared with BRCA1-transfected cells (Fig. 4A).

To further illustrate the chromosome aberrations induced by irofulven, FISH analysis was carried out in vector-transfected HCC1937 metaphase cells with the whole chromosome 1 FISH paint probe. Images again revealed that irofulven induces a significant amount of chromatid/chromosome breaks and radials involving chromosome 1 (Fig. 4B). These results suggest that the repair of irofulven-induced DSBs is largely impaired in BRCA1-deficient cells, and BRCA1 plays a pivotal role in maintaining chromosome integrity in response to irofulven-induced DNA damage.

To verify the role that BRCA1 plays in maintaining chromosome integrity, and especially in chemosensitivity in response to irofulven-elicited DNA damage, we employed the RNA interference approach to stably knock-down BRCA1 in SKOV3 cells. 
The effectiveness of three sh-BRCA1 (short-hairpin BRCA1) constructs (sh-B1, sh-B2 and sh-B3) in knocking-down the endogenous BRCA1 levels was determined by Western blot. As shown in Fig. 5A, the sh-B2 most effectively reduced BRCA1 protein level, therefore, was chosen for subsequent studies.

Chromosome aberrations were again evaluated in BRCA1-depleted mitotic SKOV3 cells. As shown in Fig. 5B, chromosome breaks were increased in untreated shBRCA1 (sh-B2)-transfected SKOV3 cells, which was further exacerbated after irofulven treatment (Fig. 5B). Strikingly, the majority of sh-B2-transfected SKOV3 cells displayed more severe chromosome damage after treatment. In these metaphase cells, chromosomes were damaged to the point where all of the chromosomes appeared to be broken or fragmented (Fig. 5C).

FISH analysis was also performed in sh-B2-transfected SKOV3 metaphase cells with the whole chromosome 1 FISH paint probe. Similar to what was observed above, images displayed extensive fragmentation of chromosome 1 after irofulven treatment (Fig. 5D). Taken together, these results demonstrate that BRCA1 plays an important role in maintaining chromosome integrity in response to irofulven-induced DNA damage.

\section{BRCA1 confers chemoresistance to irofulven.}

To determine whether BRCA1 might affect chemosensitivity to irofulven, the clonogenic survival assay was carried out. The vector and BRCA1-transfected HCC1937 cells were treated with different concentrations of irofulven for one hour followed by drug-free incubations. When IC50 concentrations were compared, the results demonstrated that the vector-transfected cells were two-fold more sensitive than the 
BRCA1-transfected cells (Fig. 6A). We also conducted clonogenic assay with longer exposure time to verify that BRCA1-deficient cells are more sensitive. The results indicated that at $0.25 \mu \mathrm{M}$, a concentration that caused no difference in chemosensitivity between the vector and BRCA1-transfected HCC1937 cells in Fig. 6A, the BRCA1transfected HCC1937 cells are four-fold more resistant when treated for six hours, and 19-fold more resistant when treated for 24 hours, than vector-transfected HCC1937 cells (Fig. 6B). These results demonstrate that BRCA1 deficiency renders cancer cells more sensitive to irofulven.

To corroborate these results, clonogenic survival assay was also performed in the vector and sh-BRCA1 transfected SKOV3 cells. Cells were treated with different concentrations of irofulven for one hour followed by drug-free incubations. The results demonstrated that knocking-down the endogenous BRCA1 levels resulted in more than two-fold increase in chemosensitivity to irofulven when IC50 concentrations were compared (Fig. 6C). Therefore, it can be concluded that BRCA1 confers chemoresistance to irofulven. 


\section{DISCUSSION}

In this study, we have observed that irofulven induces the formation of chromosome breaks and radials as well as the formation of $\gamma$-H2AX, RAD51 and BRCA1 foci. We have also provided evidence that irofulven induces the generation of DSBs. Furthermore, we have demonstrated that BRCA1 contributes to the control of S and G2/M cell cycle arrests, and is critical for RAD51-dependent HR repair of DSBs, chromosome integrity and chemosensitivity in response to irofulven.

$B R C A 1$ is frequently mutated in familial breast and ovarian cancer. Cancers that arise in mutation carriers have often lost the second allele through somatic alterations that must occur during tumor progression (1-4). It has previously been shown in several tumor cell lines that continuous exposure to irofulven resulted in a few to several hundred-fold difference in cytotoxicity based solely on increased exposure times (42-44). We found that BRCA1-deficient cells are more sensitive to irofulven treatment. We also found that when being treated for a longer period of time at lower concentration, greater sensitivity can be reached in BRCA1-deficient cells. This suggests that by maintaining a low level of drug through consecutive exposures to irofulven, BRCA1 deficiency might be exploited clinically to achieve preferential therapeutic outcomes.

BRCA1 plays an important role in regulating cell cycle checkpoints after IR (1, 2). However, a recent study demonstrated that BRCA1-deficient MEFs arrested at $\mathrm{S}$ and G2/M phases in response to mitomycin C treatment (45). In this study we have found that BRCA1 controls both $\mathrm{S}$ and G2/M checkpoints in response to irofulven-induced DNA damage. 
To date, the structure and nature of irofulven-induced DNA damage have not been fully characterized. Earlier studies have suggested that the DNA damage caused by the illudin family of compounds might be repaired by the NER pathway $(20,21)$. Recent studies suggested that TC-NER was the exclusive repair pathway in repairing illudin $\mathrm{S}$ and irofulven-elicited DNA lesions and that irofulven cytotoxicity was influenced by the expression of excision endonuclease XPG $(22,23)$. However, the HR pathway for DSB repair was not evaluated in these studies. In this study, we have provided evidence that irofulven induces the generation of DSBs, and BRCA1 plays an important role in RAD51-dependent HR repair, chromosome integrity and chemosensitivity in response to irofulven-induced DSBs. These findings are consistent with our previous observations that irofulven induces the activation of ATM and its target genes NBS1, SMC1 and CHK2 (29). A distinct possibility exists that irofulven is able to produce multiple types of DNA lesions. Since BRCA1 plays important roles in multiple DNA repair pathways including HR and TC-NER $(1,2,5)$, it remains to be determined whether BRCA1 might also be involved in TC-NER of irofulven-induced DNA lesions.

Notably, we observed that there was some degree of RAD51 foci formation in vector-transfected-HCC1937 cells after irofulven treatment. HCC1937 cells lack the wild-type allele but retain the mutant allele (5382insC) of BRCA1. As a result, this cell line expresses a BRCA1 protein truncated at amino acid 1755 of the C-terminus, resulting in loss of the second BRCT domain $(30,32,40,46)$. Due to its known BRCA1 mutation status, this cell line is widely used for the study of BRCA1 functions $(6,30,32,40,46$, 47). Earlier investigations have demonstrated that in response to DNA damage, BRCA1 forms a large protein complex with a group of proteins including MSH2, MSH6, MLH1, 
ATM, BLM, and the MRE11-RAD50-NBS1 (M/R/N) protein complex, indicating that BRCA1 may function as a coordinator of multiple activities required for the maintenance of genomic integrity and DNA repair (48-50). Recent studies indicate that in response to DSBs, BRCA1 complexes with multiple protein partners, BRCA1/BARD1/BACH1/TopBP1, BRCA1/BARD1/CtIP/M/R/N or BRCA1/BARD1/BRCA2/RAD51, integrating the activities of these partners in cell cycle checkpoints and HR repair of DSBs $(6,40)$. Recent studies also demonstrate that the tandem BRCT domains of BRCA1 function as phospho-serine- or phospho-threoninespecific binding modules that recognize substrates phosphorylated by ATM. The two BRCT domains of BRCA1, but not the individual BRCT domains alone, displayed phospho-specific binding $(46,51)$. Therefore, the impaired RAD51 foci formation in HCC1937 cells in response to irofulven- or IR/laser-induced DSBs observed in this and other studies (40), could be due to the decrease in binding of partner proteins, such as BRCA2, which is critical for RAD51 foci formation $(6,40,47,52-56)$. It could also be due to the impaired interaction with the $\mathrm{M} / \mathrm{R} / \mathrm{N}$ complex at the DSB sites $(48-50)$ or to the loss of interaction with the ATM-phosphorylated substrates, such as BACH1 $(40,46)$. In support of this, HCC1937 cells displayed barely detectable association of BRCA1associated proteins, BARD1, RAD51, BRCA2, and BACH1; and decreased association of truncated BRCA1 with TopBP1 (albeit not absent), compared with BRCA1transfected HCC1937 cells in response to DSBs (40). In addition, some degree of RAD51 foci formation was also observed in mouse BRCA1-/- (deleted for exon 11) ES cells compared with BRCA1+/+ ES cells in response to IR-induced DSBs (45). 
The chromosome breaks, tri-radials and quadri-radials formed after irofulven treatment are reminiscent of Fanconi anemia and BRCA2-deficient cells treated with IR or mitomycin $\mathrm{C}(1,3,5)$. Based on the roles that BRCA1 plays in RAD51-dependent HR, chromosome integrity and chemosensitivity in response to irofulven, it can be postulated that cells deficient in other important proteins involved in the HR pathway of DSB repair, such as FANCD2, BRCA2 or RAD51, might also show increased sensitivity. FANCD2 and $B R C A 2$ are found frequently mutated or repressed in many types of cancers $(1,3,5$, $57)$.

In summary, we have observed that irofulven induces the formation of chromosome breaks and radials as well as the formation of $\gamma$-H2AX, RAD51 and BRCA1 foci. We have also provided evidence that irofulven induces the generation of DSBs. We have demonstrated that BRCA1 is critical for S and G2/M phase cell cycle checkpoints, RAD51-dependent HR, chromosome integrity and chemosensitivity in response to irofulven. These findings will enhance our understanding of the mechanisms of action involved with irofulven, and more specifically, the proteins and mechanisms that might affect irofulven-induced chemosensitivity. They will also provide insight for future studies of targeted therapy by irofulven in BRCA1-deficient familial breast and ovarian cancers. 


\section{ACKNOWLEDGMENTS}

We would like to thank Prof. Ralph Scully (Dana-Farber Cancer Institute, Harvard Medical School, Boston, MA) for generously providing the vector and BRCA1transfected HCC1937 cells. We also thank Dr. Mike Miller for the help on PFGE, Dr. Linda Sargent for the use of fluorescent microscopes, and Emily Van Laar and Shannon Wadman for critical reading of the manuscript. This work was supported in part by a grant from the National Cancer Institute (R03CA107979), a grant from MGI Pharma, Inc. and funding from the Fannie Rippel Foundation (to W. W.). 


\section{REFERENCES}

1. Venkitaraman, A. R. Tracing the network connecting BRCA and Fanconi anaemia proteins. Nat Rev Cancer, 4: 266-276, 2004.

2. Narod, S. A. and Foulkes, W. D. BRCA1 and BRCA2: 1994 and beyond. Nat Rev Cancer, 4: 665-676, 2004.

3. Venkitaraman, A. R. Cancer susceptibility and the functions of BRCA1 and BRCA2. Cell, 108: 171-182, 2002.

4. Zhou, B. B. and Elledge, S. J. The DNA damage response: putting checkpoints in perspective. Nature, 408: 433-439, 2000.

5. D'Andrea, A. D. and Grompe, M. The Fanconi anaemia/BRCA pathway. Nat Rev Cancer, 3: 23-34, 2003.

6. Scully, R., Chen, J., Plug, A., Xiao, Y., Weaver, D., Feunteun, J., Ashley, T., and Livingston, D. M. Association of BRCA1 with Rad51 in mitotic and meiotic cells. Cell, 88: 265-275, 1997.

7. Riballo, E., Kuhne, M., Rief, N., Doherty, A., Smith, G. C., Recio, M. J., Reis, C., Dahm, K., Fricke, A., Krempler, A., Parker, A. R., Jackson, S. P., Gennery, A., Jeggo, P. A., and Lobrich, M. A pathway of double-strand break rejoining dependent upon ATM, Artemis, and proteins locating to gamma-H2AX foci. Mol Cell, 16: 715-724, 2004. 
8. Morrison, A. J., Highland, J., Krogan, N. J., Arbel-Eden, A., Greenblatt, J. F., Haber, J. E., and Shen, X. INO80 and gamma-H2AX interaction links ATPdependent chromatin remodeling to DNA damage repair. Cell, 119: 767-775, 2004.

9. van Attikum, H., Fritsch, O., Hohn, B., and Gasser, S. M. Recruitment of the INO80 complex by H2A phosphorylation links ATP-dependent chromatin remodeling with DNA double-strand break repair. Cell, 119: 777-788, 2004.

10. Xie, A., Puget, N., Shim, I., Odate, S., Jarzyna, I., Bassing, C. H., Alt, F. W., and Scully, R. Control of sister chromatid recombination by histone H2AX. Mol Cell, 16: 1017-1025, 2004.

11. MacDonald, J. R., Muscoplat, C. C., Dexter, D. L., Mangold, G. L., Chen, S. F., Kelner, M. J., McMorris, T. C., and Von Hoff, D. D. Preclinical antitumor activity of 6-hydroxymethylacylfulvene, a semisynthetic derivative of the mushroom toxin illudin S. Cancer Res, 57: 279-283, 1997.

12. Sato, Y., Kashimoto, S., MacDonald, J. R., and Nakano, K. In vivo antitumour efficacy of MGI-114 (6-hydroxymethylacylfulvene, HMAF) in various human tumour xenograft models including several lung and gastric tumours. Eur J Cancer, 37: 1419-1428, 2001.

13. Friedman, H. S., Keir, S. T., Houghton, P. J., Lawless, A. A., Bigner, D. D., and Waters, S. J. Activity of irofulven (6-hydroxymethylacylfulvene) in the treatment 
of glioblastoma multiforme-derived xenografts in athymic mice. Cancer Chemother Pharmacol, 48: 413-416, 2001.

14. Hidalgo, M., Izbicka, E., Eckhardt, S. G., MacDonald, J. R., Cerna, C., Gomez, L., Rowinsky, E. K., Weitman, S. D., and Von Hoff, D. D. Antitumor activity of MGI 114 (6-hydroxymethylacylfulvene, HMAF), a semisynthetic derivative of illudin S, against adult and pediatric human tumor colony-forming units. Anticancer Drugs, 10: 837-844, 1999.

15. Britten, C. D., Hilsenbeck, S. G., Eckhardt, S. G., Marty, J., Mangold, G., MacDonald, J. R., Rowinsky, E. K., Von Hoff, D. D., and Weitman, S. Enhanced antitumor activity of 6-hydroxymethylacylfulvene in combination with irinotecan and 5-fluorouracil in the HT29 human colon tumor xenograft model. Cancer Res, 59: 1049-1053, 1999.

16. Murgo, A., Cannon, D. J., Blatner, G., and Cheson, B. D. Clinical trials referral resource. Clinical trials of MGI-114. Oncology (Huntingt), 13: 233, 237-238, 1999.

17. Kelner, M. J., McMorris, T. C., Rojas, R. J., Trani, N. A., Velasco, T. R., Estes, L. A., and Suthipinijtham, P. Enhanced antitumor activity of irofulven in combination with antimitotic agents. Invest New Drugs, 20: 271-279, 2002.

18. Senzer, N., Arsenau, J., Richards, D., Berman, B., MacDonald, J. R., and Smith, S. Irofulven demonstrates clinical activity against metastatic hormone-refractory prostate cancer in a phase 2 single-agent trial. Am J Clin Oncol, 28: 36-42, 2005. 
19. Woo, M. H., Peterson, J. K., Billups, C., Liang, H., Bjornsti, M. A., and Houghton, P. J. Enhanced antitumor activity of irofulven in combination with irinotecan in pediatric solid tumor xenograft models. Cancer Chemother Pharmacol, 55: 411-419, 2005.

20. Kelner, M. J., McMorris, T. C., Estes, L., Rutherford, M., Montoya, M., Goldstein, J., Samson, K., Starr, R., and Taetle, R. Characterization of illudin S sensitivity in DNA repair-deficient Chinese hamster cells. Unusually high sensitivity of ERCC2 and ERCC3 DNA helicase-deficient mutants in comparison to other chemotherapeutic agents. Biochem Pharmacol, 48: 403-409, 1994.

21. Kelner, M. J., McMorris, T. C., Estes, L., Starr, R. J., Rutherford, M., Montoya, M., Samson, K. M., and Taetle, R. Efficacy of Acylfulvene Illudin analogues against a metastatic lung carcinoma MV522 xenograft nonresponsive to traditional anticancer agents: retention of activity against various mdr phenotypes and unusual cytotoxicity against ERCC2 and ERCC3 DNA helicase-deficient cells. Cancer Res, 55: 4936-4940, 1995.

22. Jaspers, N. G., Raams, A., Kelner, M. J., Ng, J. M., Yamashita, Y. M., Takeda, S., McMorris, T. C., and Hoeijmakers, J. H. Anti-tumour compounds illudin S and Irofulven induce DNA lesions ignored by global repair and exclusively processed by transcription- and replication-coupled repair pathways. DNA Repair (Amst), 1: 1027-1038, 2002. 
23. Koeppel, F., Poindessous, V., Lazar, V., Raymond, E., Sarasin, A., and Larsen, A. K. Irofulven cytotoxicity depends on transcription-coupled nucleotide excision repair and is correlated with XPG expression in solid tumor cells. Clin Cancer Res, 10: 5604-5613, 2004.

24. Bakkenist, C. J. and Kastan, M. B. DNA damage activates ATM through intermolecular autophosphorylation and dimer dissociation. Nature, 421: 499-506, 2003.

25. Lee, J. H. and Paull, T. T. Direct activation of the ATM protein kinase by the Mre11/Rad50/Nbs1 complex. Science, 304: 93-96, 2004.

26. Lee, J. H. and Paull, T. T. ATM activation by DNA double-strand breaks through the Mre11-Rad50-Nbs1 complex. Science, 308: 551-554, 2005.

27. Ismail, I. H., Nystrom, S., Nygren, J., and Hammarsten, O. Activation of ataxia telangiectasia mutated by DNA strand break-inducing agents correlates closely with the number of DNA double strand breaks. J Biol Chem, 280: 4649-4655, 2005.

28. Buscemi, G., Perego, P., Carenini, N., Nakanishi, M., Chessa, L., Chen, J., Khanna, K., and Delia, D. Activation of ATM and Chk2 kinases in relation to the amount of DNA strand breaks. Oncogene, 23: 7691-7700, 2004.

29. Wang, J., Wiltshire, T., Wang, Y., Mikell, C., Burks, J., Cunningham, C., Van Laar, E. S., Waters, S. J., Reed, E., and Wang, W. ATM-dependent CHK2 
Activation Induced by Anticancer Agent, Irofulven. J Biol Chem, 279: 3958439592, 2004.

30. Scully, R., Ganesan, S., Vlasakova, K., Chen, J., Socolovsky, M., and Livingston, D. M. Genetic analysis of BRCA1 function in a defined tumor cell line. Mol Cell, 4: 1093-1099, 1999.

31. Xu, B., Kim, S. T., Lim, D. S., and Kastan, M. B. Two molecularly distinct G(2)/M checkpoints are induced by ionizing irradiation. Mol Cell Biol, 22: 10491059, 2002.

32. Tomlinson, G. E., Chen, T. T., Stastny, V. A., Virmani, A. K., Spillman, M. A., Tonk, V., Blum, J. L., Schneider, N. R., Wistuba, II, Shay, J. W., Minna, J. D., and Gazdar, A. F. Characterization of a breast cancer cell line derived from a germ-line BRCA1 mutation carrier. Cancer Res, 58: 3237-3242, 1998.

33. Husain, A., He, G., Venkatraman, E. S., and Spriggs, D. R. BRCA1 up-regulation is associated with repair-mediated resistance to cisdiamminedichloroplatinum(II). Cancer Res, 58: 1120-1123, 1998.

34. Paull, T. T., Rogakou, E. P., Yamazaki, V., Kirchgessner, C. U., Gellert, M., and Bonner, W. M. A critical role for histone $\mathrm{H} 2 \mathrm{AX}$ in recruitment of repair factors to nuclear foci after DNA damage. Curr Biol, 10: 886-895, 2000. 
35. Rothkamm, K. and Lobrich, M. Evidence for a lack of DNA double-strand break repair in human cells exposed to very low x-ray doses. Proc Natl Acad Sci U S A, 100: 5057-5062, 2003.

36. Sedelnikova, O. A., Rogakou, E. P., Panyutin, I. G., and Bonner, W. M. Quantitative detection of (125)IdU-induced DNA double-strand breaks with gamma-H2AX antibody. Radiat Res, 158: 486-492, 2002.

37. Rogakou, E. P., Pilch, D. R., Orr, A. H., Ivanova, V. S., and Bonner, W. M. DNA double-stranded breaks induce histone H2AX phosphorylation on serine 139. J Biol Chem, 273: 5858-5868, 1998.

38. Franco, S., Gostissa, M., Zha, S., Lombard, D. B., Murphy, M. M., Zarrin, A. A., Yan, C., Tepsuporn, S., Morales, J. C., Adams, M. M., Lou, Z., Bassing, C. H., Manis, J. P., Chen, J., Carpenter, P. B., and Alt, F. W. H2AX prevents DNA breaks from progressing to chromosome breaks and translocations. Mol Cell, 21: 201-214, 2006.

39. Stucki, M., Clapperton, J. A., Mohammad, D., Yaffe, M. B., Smerdon, S. J., and Jackson, S. P. MDC1 directly binds phosphorylated histone H2AX to regulate cellular responses to DNA double-strand breaks. Cell, 123: 1213-1226, 2005.

40. Greenberg, R. A., Sobhian, B., Pathania, S., Cantor, S. B., Nakatani, Y., and Livingston, D. M. Multifactorial contributions to an acute DNA damage response by BRCA1/BARD1-containing complexes. Genes Dev, 20: 34-46, 2006. 
41. West, S. C. Molecular views of recombination proteins and their control. Nat Rev Mol Cell Biol, 4: 435-445, 2003.

42. Kelner, M. J., McMorris, T. C., Montoya, M. A., Estes, L., Rutherford, M., Samson, K. M., and Taetle, R. Characterization of cellular accumulation and toxicity of illudin $\mathrm{S}$ in sensitive and nonsensitive tumor cells. Cancer Chemother Pharmacol, 40: 65-71, 1997.

43. Kelner, M. J., McMorris, T. C., Montoya, M. A., Estes, L., Uglik, S. F., Rutherford, M., Samson, K. M., Bagnell, R. D., and Taetle, R. Characterization of MGI 114 (HMAF) histiospecific toxicity in human tumor cell lines. Cancer Chemother Pharmacol, 44: 235-240, 1999.

44. Kelner, M. J., McMorris, T. C., and Taetle, R. Preclinical evaluation of illudins as anticancer agents: basis for selective cytotoxicity. J Natl Cancer Inst, 82: 15621565,1990 .

45. Bhattacharyya, A., Ear, U. S., Koller, B. H., Weichselbaum, R. R., and Bishop, D. $\mathrm{K}$. The breast cancer susceptibility gene BRCA1 is required for subnuclear assembly of Rad51 and survival following treatment with the DNA cross-linking agent cisplatin. J Biol Chem, 275: 23899-23903, 2000.

46. Yu, X., Chini, C. C., He, M., Mer, G., and Chen, J. The BRCT domain is a phospho-protein binding domain. Science, 302: 639-642, 2003. 
47. Chen, J., Silver, D. P., Walpita, D., Cantor, S. B., Gazdar, A. F., Tomlinson, G., Couch, F. J., Weber, B. L., Ashley, T., Livingston, D. M., and Scully, R. Stable interaction between the products of the BRCA1 and BRCA2 tumor suppressor genes in mitotic and meiotic cells. Mol Cell, 2: 317-328, 1998.

48. Zhong, Q., Chen, C. F., Li, S., Chen, Y., Wang, C. C., Xiao, J., Chen, P. L., Sharp, Z. D., and Lee, W. H. Association of BRCA1 with the hRad50-hMre11p95 complex and the DNA damage response. Science, 285: 747-750, 1999.

49. Wang, Y., Cortez, D., Yazdi, P., Neff, N., Elledge, S. J., and Qin, J. BASC, a super complex of BRCA1-associated proteins involved in the recognition and repair of aberrant DNA structures. Genes Dev, 14: 927-939, 2000.

50. Wu, X., Petrini, J. H., Heine, W. F., Weaver, D. T., Livingston, D. M., and Chen, $\mathrm{J}$. Independence of $\mathrm{R} / \mathrm{M} / \mathrm{N}$ focus formation and the presence of intact BRCA1. Science, 289: 11, 2000.

51. Manke, I. A., Lowery, D. M., Nguyen, A., and Yaffe, M. B. BRCT repeats as phosphopeptide-binding modules involved in protein targeting. Science, 302: 636-639, 2003.

52. Sharan, S. K., Morimatsu, M., Albrecht, U., Lim, D. S., Regel, E., Dinh, C., Sands, A., Eichele, G., Hasty, P., and Bradley, A. Embryonic lethality and radiation hypersensitivity mediated by Rad51 in mice lacking Brca2. Nature, 386: 804-810, 1997. 
53. Wong, A. K., Pero, R., Ormonde, P. A., Tavtigian, S. V., and Bartel, P. L. RAD51 interacts with the evolutionarily conserved BRC motifs in the human breast cancer susceptibility gene brca2. J Biol Chem, 272: 31941-31944, 1997.

54. Chen, P. L., Chen, C. F., Chen, Y., Xiao, J., Sharp, Z. D., and Lee, W. H. The BRC repeats in BRCA2 are critical for RAD51 binding and resistance to methyl methanesulfonate treatment. Proc Natl Acad Sci U S A, 95: 5287-5292, 1998.

55. Chen, J. J., Silver, D., Cantor, S., Livingston, D. M., and Scully, R. BRCA1, BRCA2, and Rad51 operate in a common DNA damage response pathway. Cancer Res, 59: 1752s-1756s, 1999.

56. Scully, R. and Livingston, D. M. In search of the tumour-suppressor functions of BRCA1 and BRCA2. Nature, 408: 429-432, 2000.

57. Turner, N., Tutt, A., and Ashworth, A. Hallmarks of 'BRCAness' in sporadic cancers. Nat Rev Cancer, 4: 814-819, 2004. 


\section{FOOTNOTES}

This work was supported in part by NIH grant R03CA107979, a grant from MGI Pharma, Inc. and funding from the Fannie Rippel Foundation (to W. W.).

Address correspondence to: Dr. Weixin Wang, Mary Babb Randolph Cancer Center, West Virginia University School of Medicine, P.O. Box 9300, Morgantown, WV 26506.

E-mail: wwang@hsc.wvu .edu.

${ }^{1}$ Present address: Center for Disease Control and Prevention, Atlanta, Georgia. 


\section{FIGURE LEGENDS}

Fig. 1. Irofulven induces chromosome breaks, triradials and quadriradials and activates BRCA1. (A) HCC1937 and SKOV3 were treated with 1xIC50 concentrations of irofulven (1.0 and $2.3 \mu \mathrm{M}$, respectively) for one hour followed by 24 hours of drug-free incubation. Pictures showed the mitotic spread staining of cells treated with irofulven. Arrows indicate chromosome breaks, triradials and quadriradials. (B) Confocal microscopic images of immunofluorescent staining for BRCA1 and $\gamma$-H2AX. SKOV3 cells were treated with 1 XIC50 concentration of irofulven for one hour followed by 12 hours of drug-free incubation.

Fig. 2. BRCA1 controls S and G2/M checkpoints in response to irofulven-induced DNA damage. (A) DNA synthesis was determined by RDS assay. The vector and BRCA1transfected HCC1937 cells were treated with 1, 2 or $3 \mu \mathrm{M}$ of irofulven for one hour followed by 12 hours of drug-free incubation. DNA synthesis rates were presented as the average and standard error of triplicate experiments. (B) The mitotic population of cells was determined by staining for phosphorylated histone H3 and flow cytometry. The vector and BRCA1-transfected HCC1937 cells were treated with $1 \mu \mathrm{M}$ of irofulven for one hour followed by one hour of drug-free incubation. The percentage of phosphohistone H3-positive population was presented as the mean and standard deviation of triplicate experiments. (C) The mitotic index of vector and BRCA1-transfected HCC1937 cells. Cells were treated with $1 \mu \mathrm{M}$ of irofulven for one hour followed by 24 hours of drug-free incubation. In each group, about 4,000 cells were counted. 
Fig. 3. BRCA1 is critical for repairing irofulven-induced DSBs and for RAD51dependent HR. The vector and BRCA1-transfected HCC1937 cells were treated with 1 $\mu \mathrm{M}$ of irofulven for one hour followed by different times of drug-free incubation. (A) Genomic DNA samples were extracted and separated by PFGE. (B and C) Comet assay was performed using neutral conditions to specifically detect double-strand breaks (B). The comet tail movement was quantified by visual scoring. The statistical significance was analyzed by Student's $t$-test and marked as * $(p<0.05)$ and $* *(p<0.01)$ (C). (D) The DNA repair dynamics was characterized by counting the $\gamma-\mathrm{H} 2 \mathrm{AX}$ foci formation. (E and F) Cells were immunofluorescently stained for RAD51, $\gamma$-H2AX and DAPI (E). Cells with five or more foci were counted as positive for staining. The percentage of cells with RAD51 or $\gamma-\mathrm{H} 2 \mathrm{AX}$ foci was exhibited as the mean and standard deviation of triplicate counts of 1,000 cells (F).

Fig. 4. Chromosome aberrations induced by irofulven are related to BRCA1 status. The vector and BRCA1-transfected HCC1937 cells were treated with $1 \mu \mathrm{M}$ of irofulven for one hour followed by 24 hours of drug-free incubation. (A) Mitotic spread staining was performed. The percentage of mitotic cells with four or more chromosome breaks or with radials was presented. In each group, 100 mitotic cells were counted. (B) FISH was performed to specifically characterize aberrations involving chromosome 1 in the vectortransfected HCC1937 cells treated with irofulven. The images show the largest portion of chromosome 1 within the HCC1937 cells (a and b). The asterisk (*) indicates an inherent 
translocation on chromosome 1. Arrows indicate the chromatid/chromosome breaks (c and $\mathbf{d )}$ and a quadri-radial (e and $\mathbf{f})$ involving chromosome 1 after irofulven treatment.

Fig. 5. Knocking-down BRCA1 protein levels by RNA interference results in increased chromosome aberrations. (A) SKOV3 cells were stably transfected with the vector (sh-V) or sh-BRCA1 (short-hairpin BRCA1) constructs (sh-B1, B2, B3), respectively. The efficacy of sh-BRCA1 constructs in knocking-down BRCA1 protein levels was determined by Western blot analysis with the anti-BRCA1 antibody. The blot for actin served as loading control. (B through D) Mitotic spread staining was performed. The shV and sh-B2-transfected SKOV3 cells were treated with 1xIC50 concentration of irofulven for one hour followed by 24 hours of drug-free incubation. The percentage of mitotic cells with four or more chromosome breaks was presented. In each group, 100 mitotic cells were counted (B). Representative picture of metaphase sh-B2-transfected SKOV3 showed widespread chromosome fragmentation after irofulven treatment (C). FISH was performed to specifically characterize the chromosome 1 damage in sh-B2transfected SKOV3 cells treated with irofulven. The images show chromosome 1 within the sh-B2-transfected SKOV3 cells (a and $\mathbf{b})$. Arrows indicate the highly altered chromosome 1 after irofulven treatment (c through f) (D).

Fig. 6. BRCA1 confers chemoresistance to irofulven. Irofulven-induced chemosensitivity was determined by clonogenic survival assay in the vector and BRCA1-transfected HCC1937 cells or in the vector and sh-BRCA1-transfected SKOV3 cells. (A through C) 
Cells were treated with irofulven for one hour (A and C), or one, six or 24 hours (B). The mean and standard deviation of triplicate experiments were demonstrated. 
Figure 1. Irofulven induces chromosome breaks, triradials and quadriradials and activates BRCA1.

\section{Figure 1A}
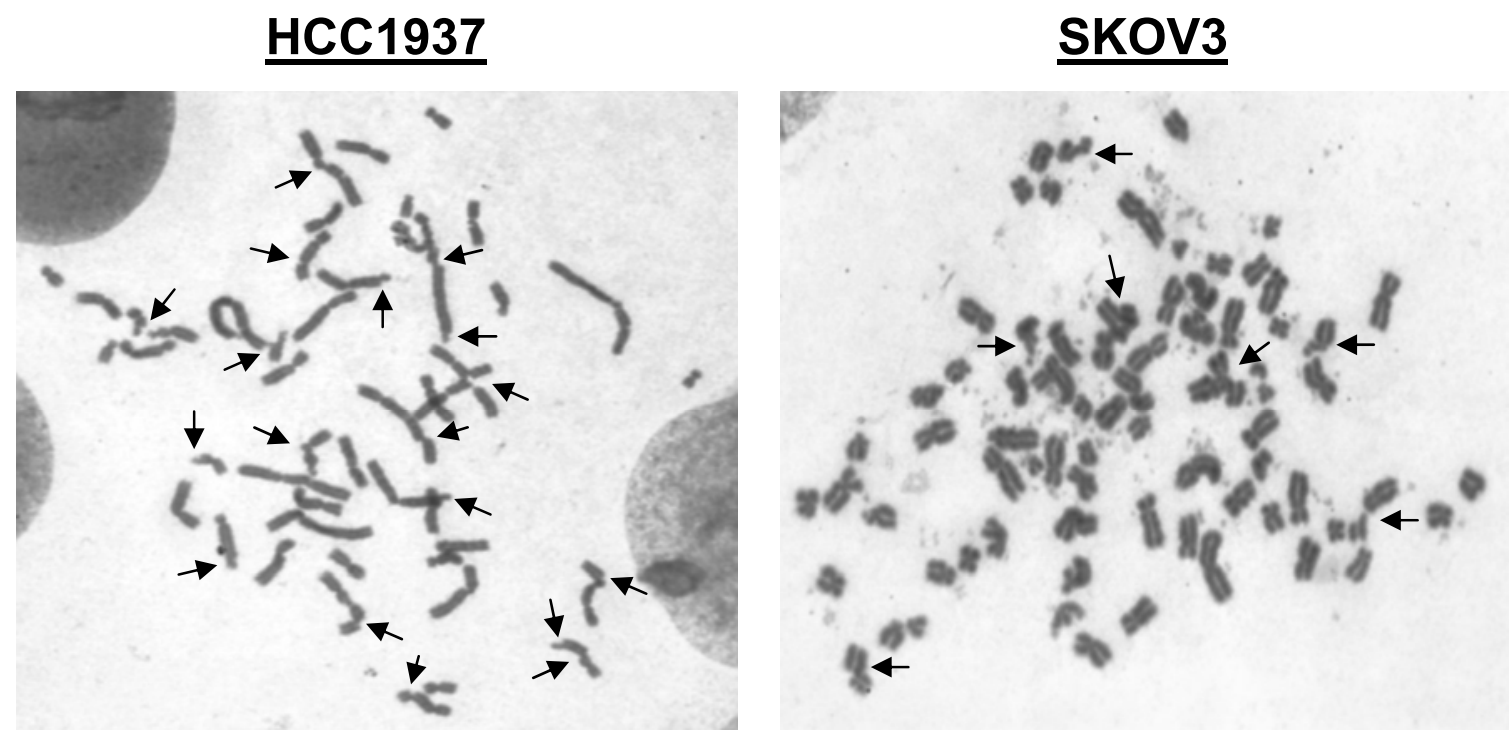

Figure 1B

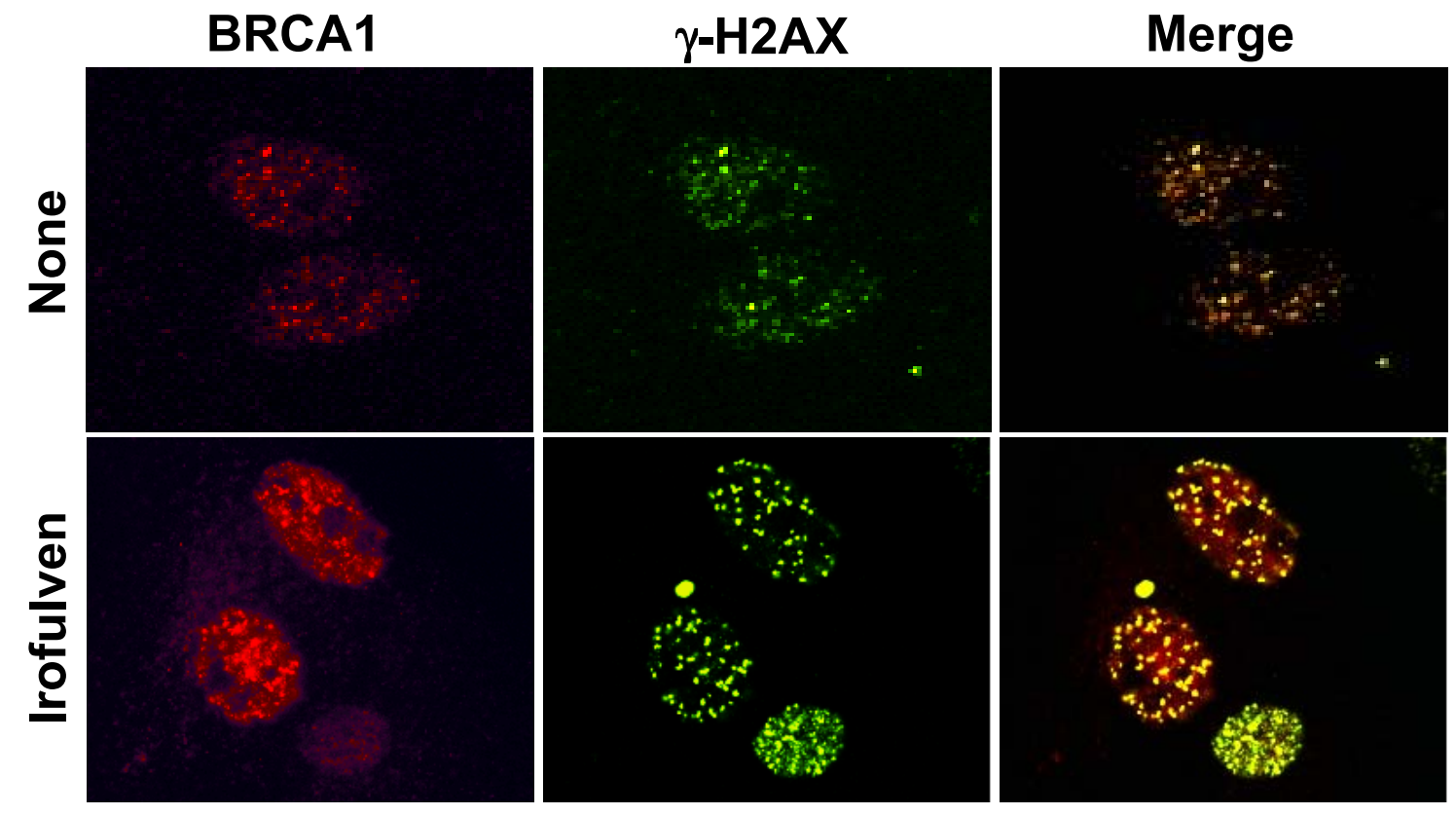


Figure 2. BRCA1 controls S and G2/M checkpoints in response to irofulven-induced DNA damage.

Figure 2A

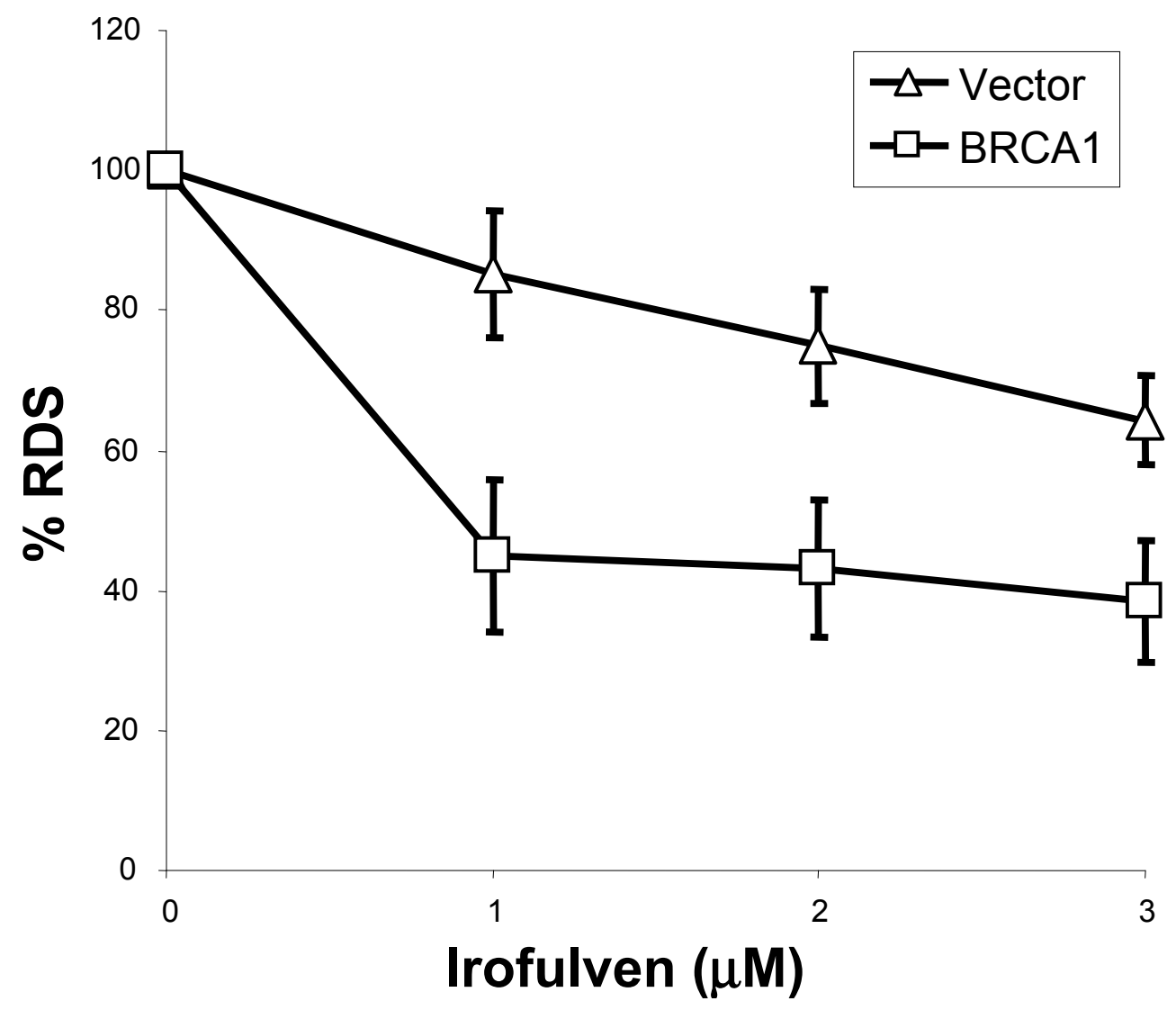


$\begin{array}{ll}\text { Figure 2B } & 1.8\end{array}$

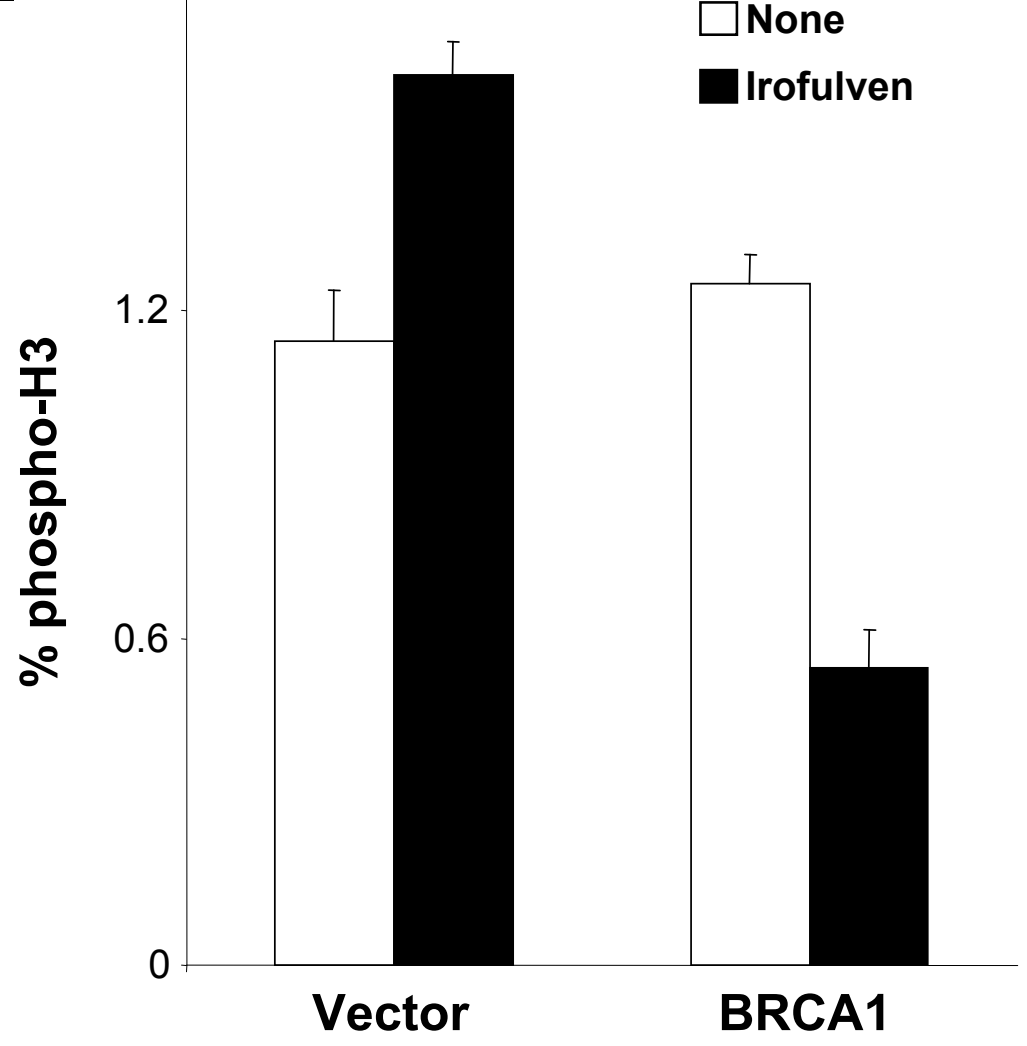


Figure 2C

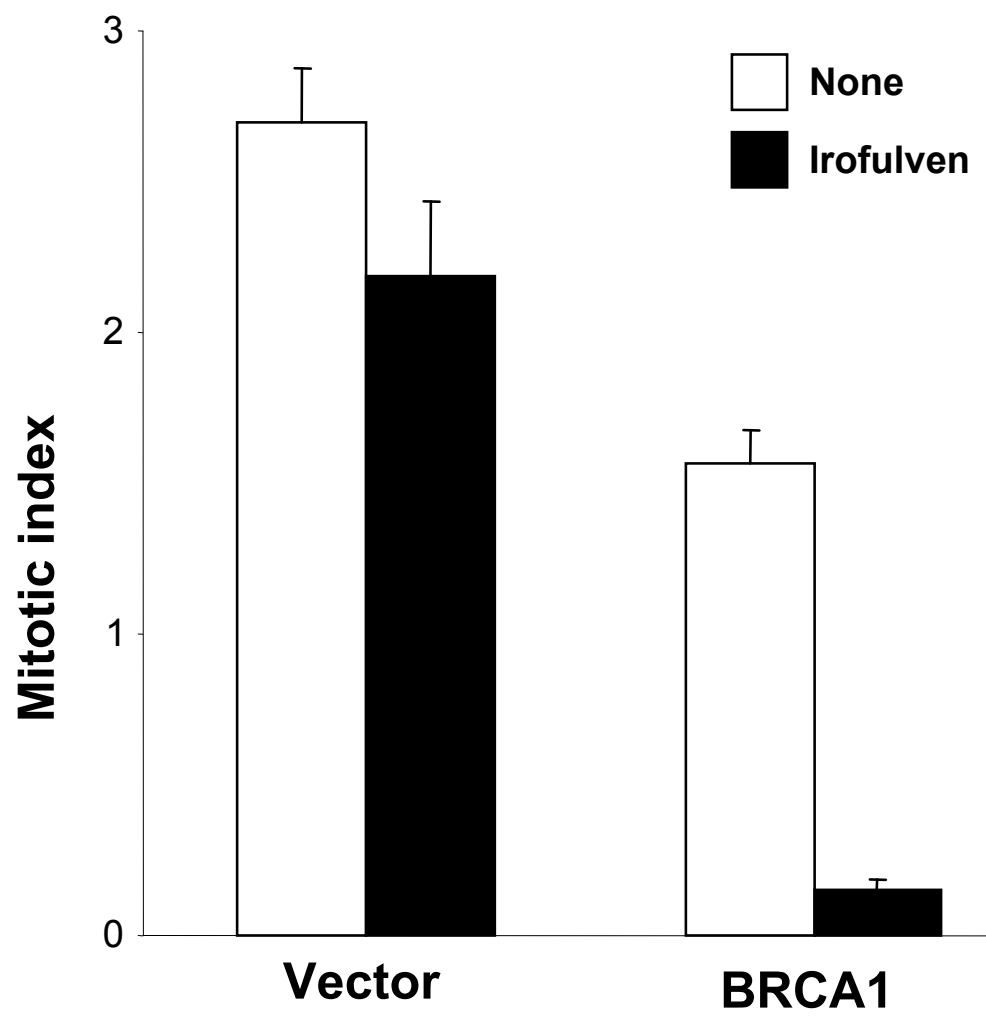


Figure 3. BRCA1 is critical for repairing irofulven-induced DSBs and for RAD51-dependent HR.

Figure 3A

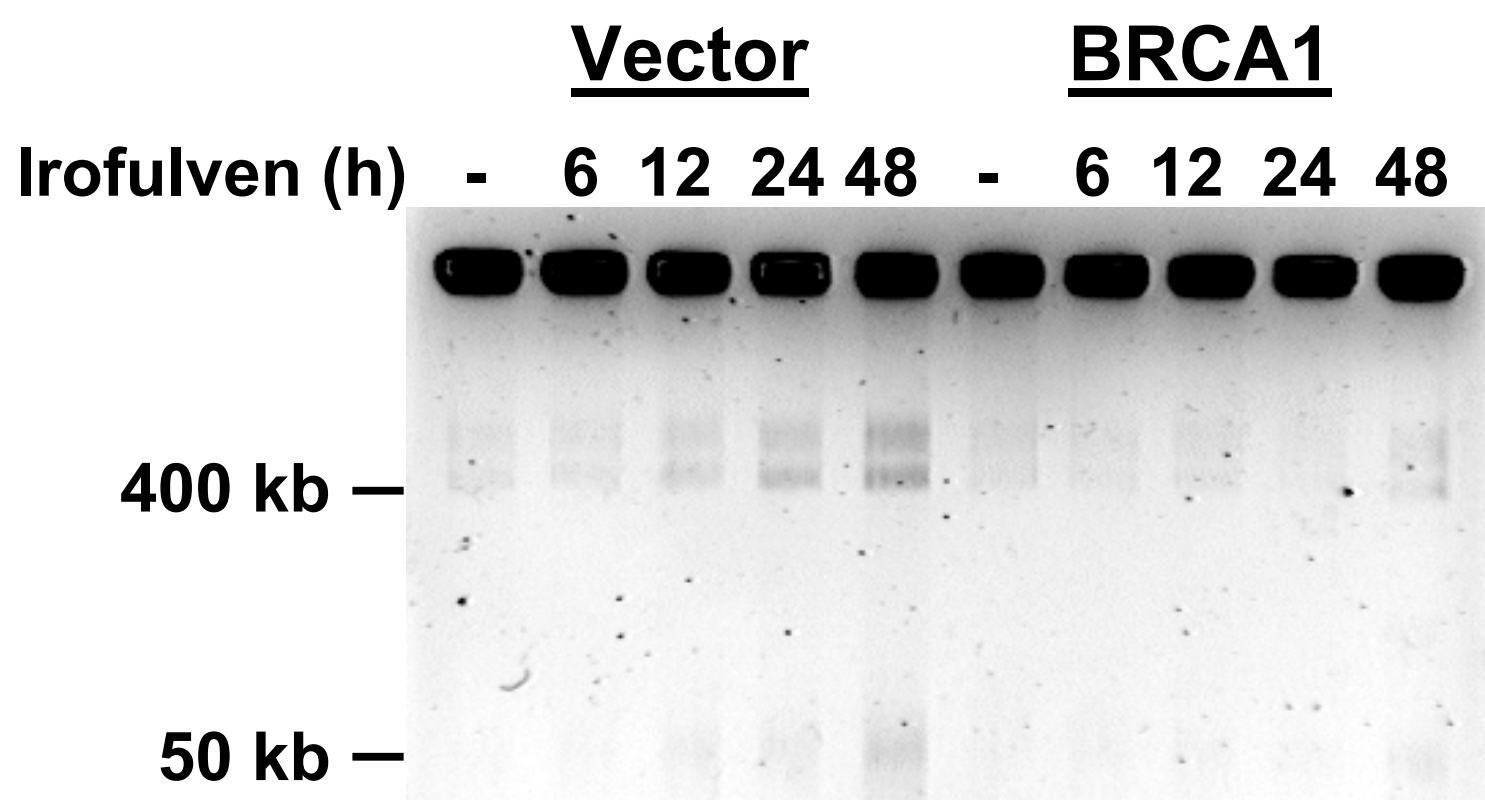


Figure 3B

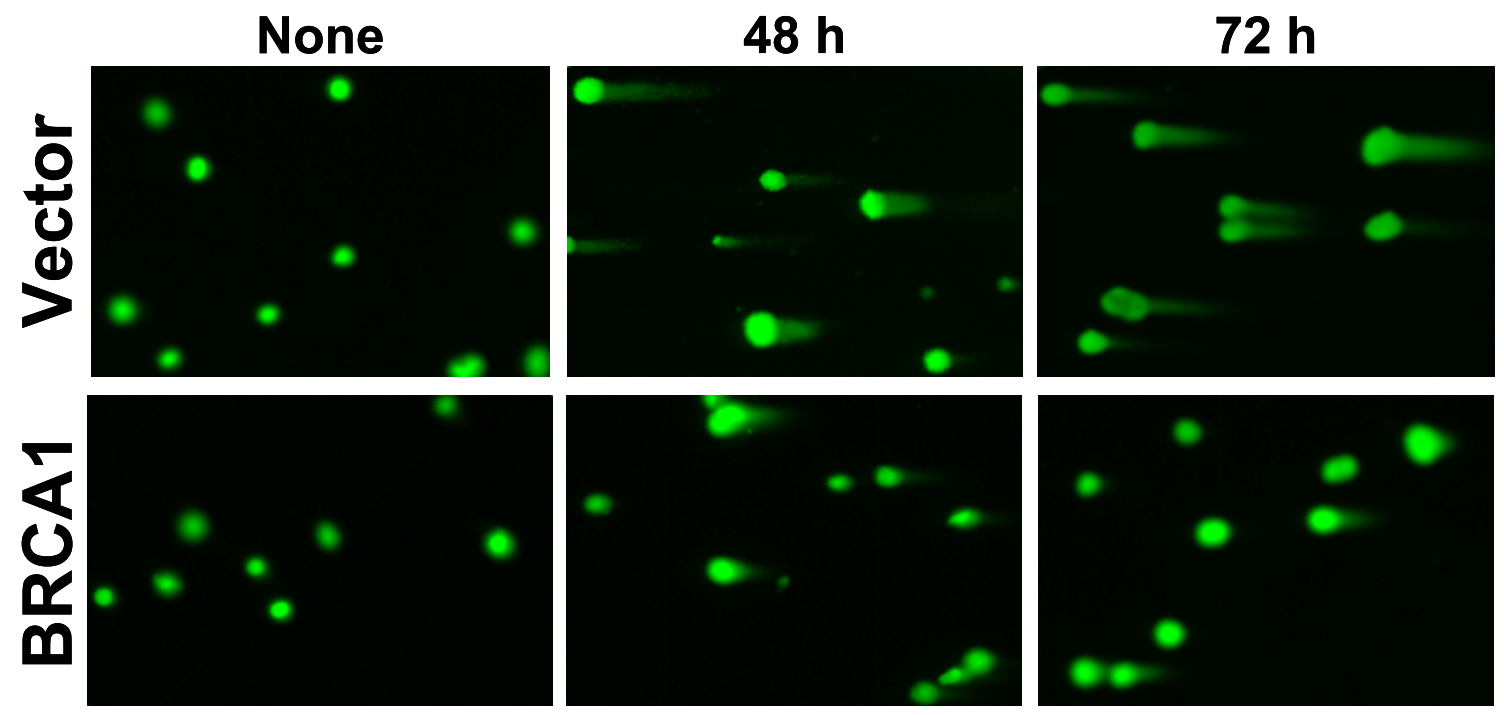

125

Figure 3C

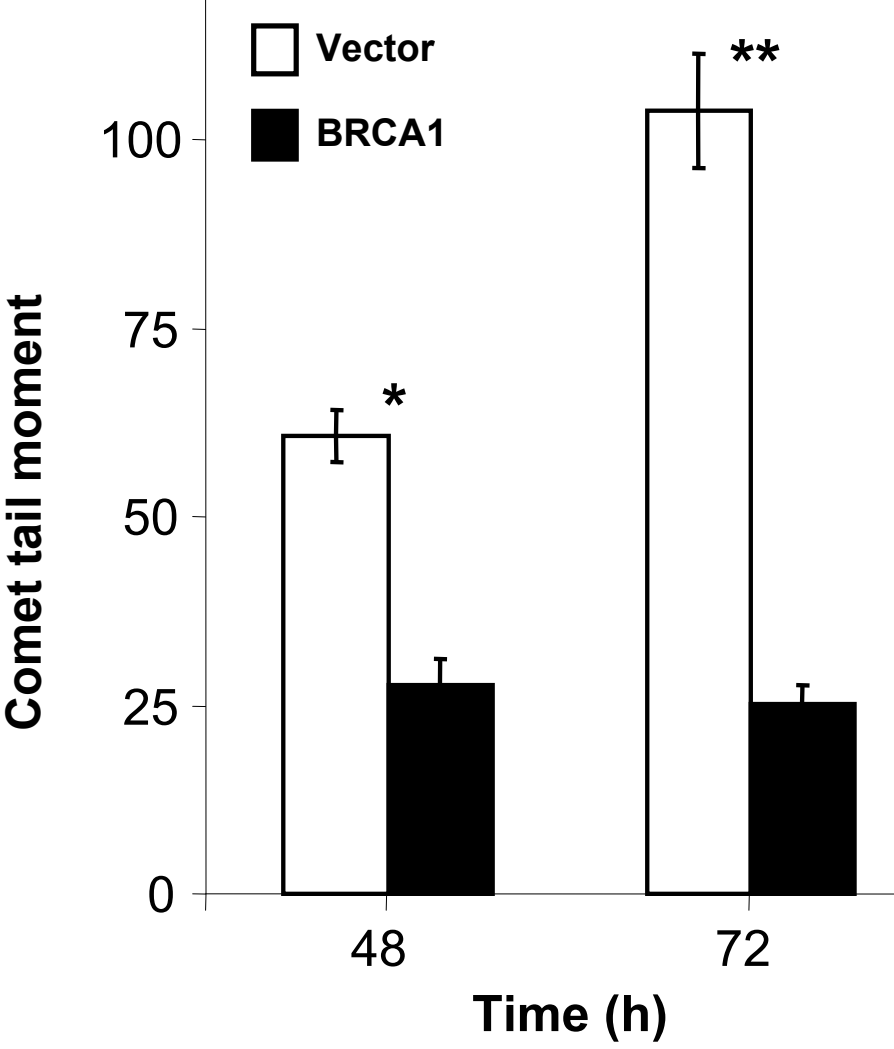


Figure 3D

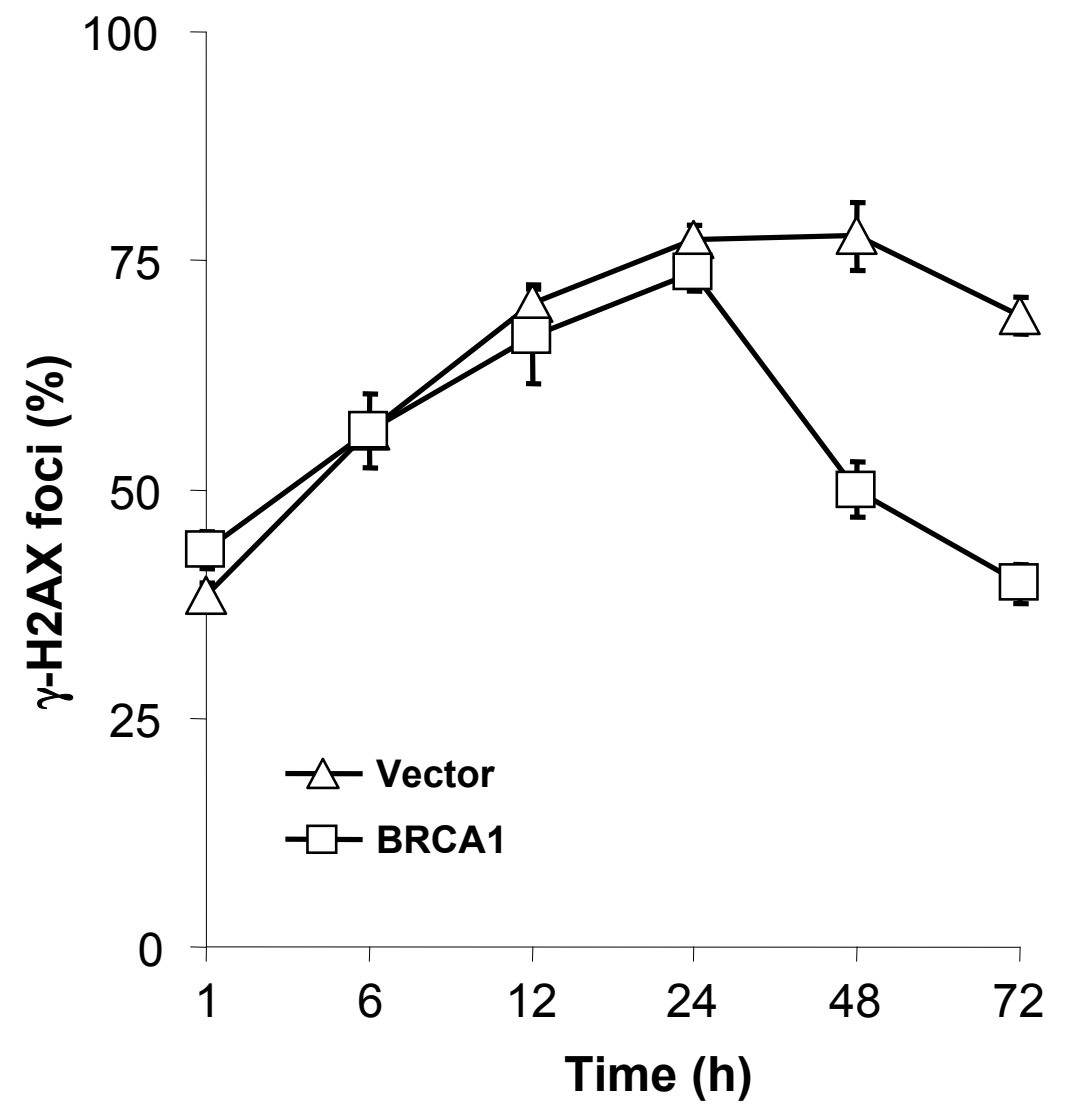


Figure 3E
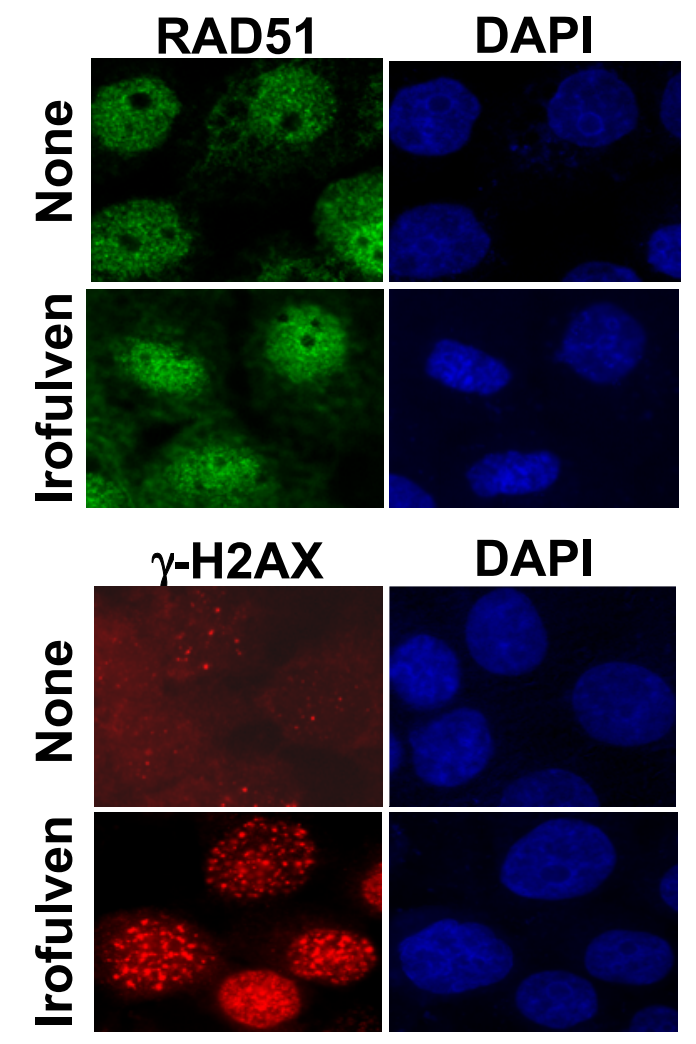

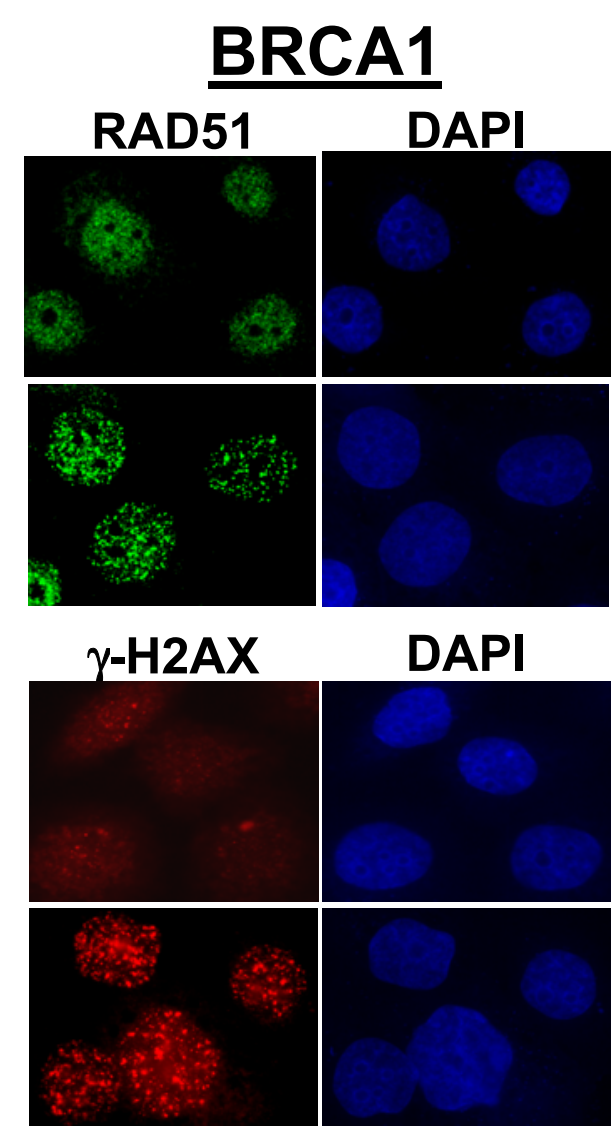

Figure 3F

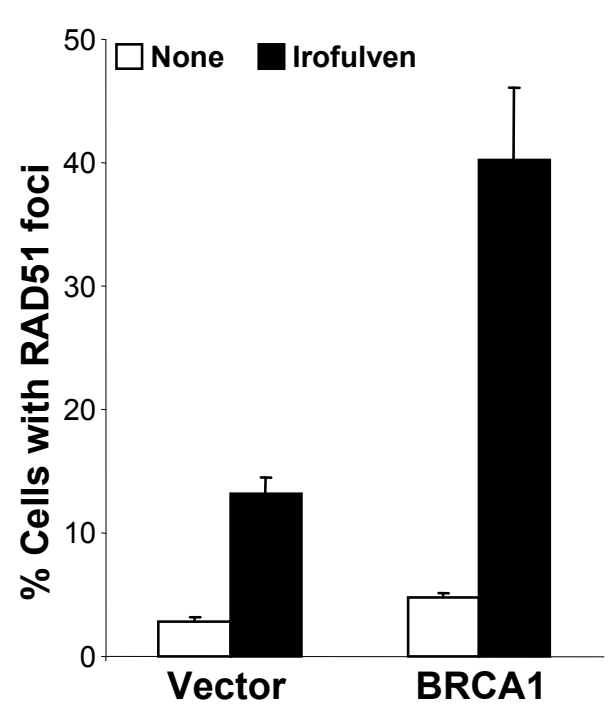


Figure 4. Chromosome aberrations induced by irofulven are related to BRCA1 status.

\section{Figure 4A}
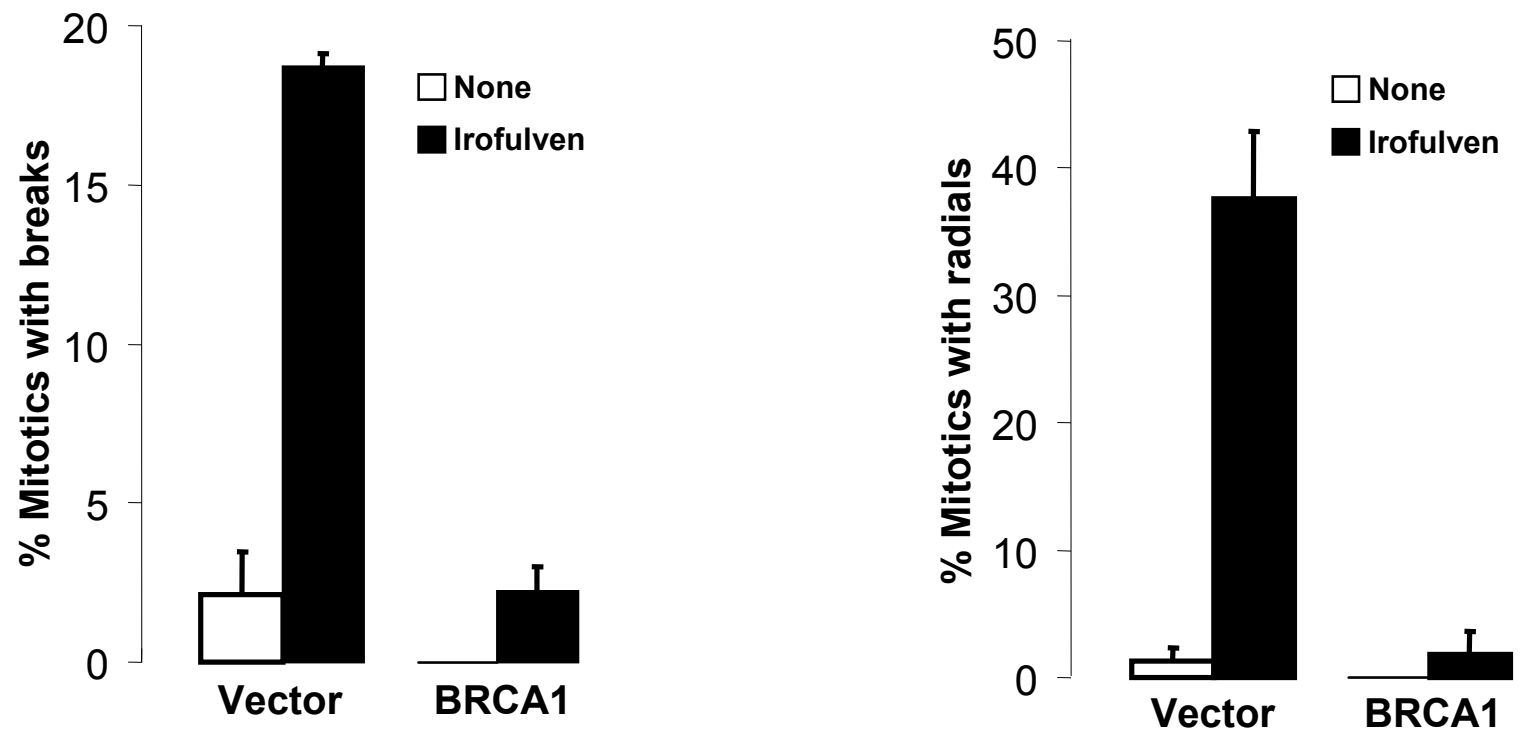

Figure 4B

Vector/HCC1937
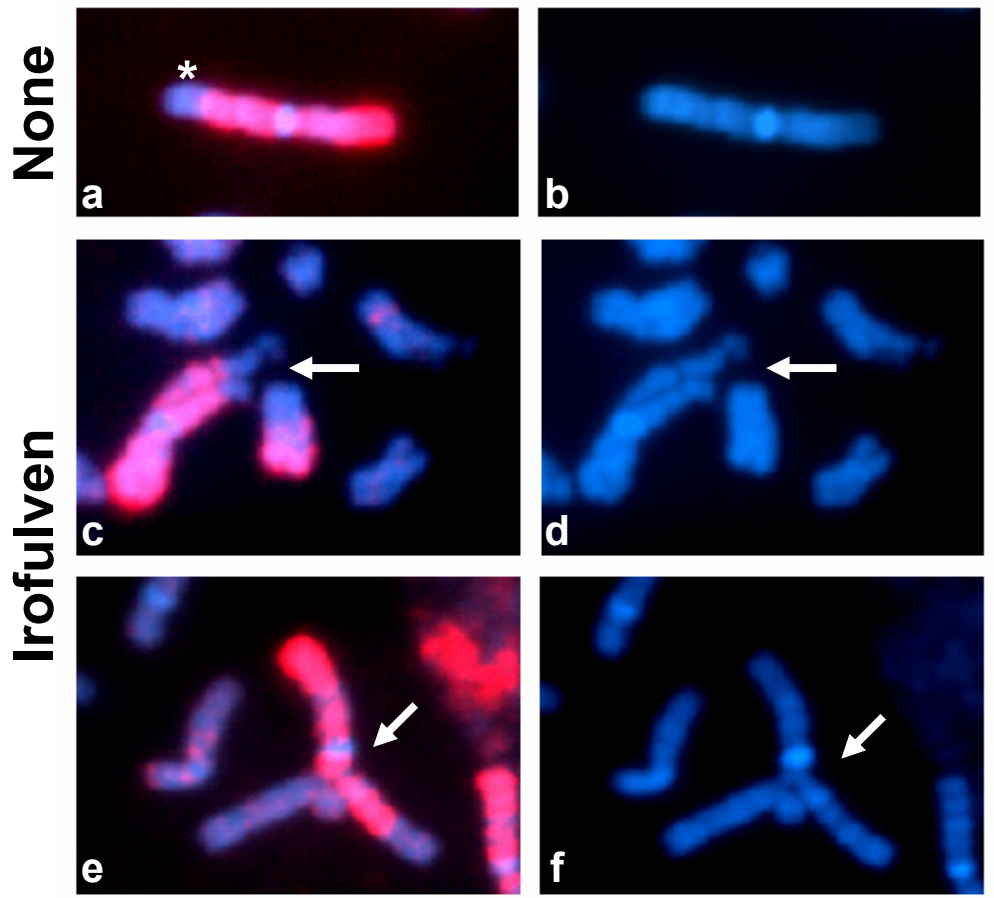
Figure 5. Knocking-down BRCA1 protein levels by RNA interference results in increased chromosome aberrations.

\title{
Figure 5A
}

\section{shRNA sh-V sh-B1 sh-B2 sh-B3 \\ BRCA1}

\author{
Actin
}

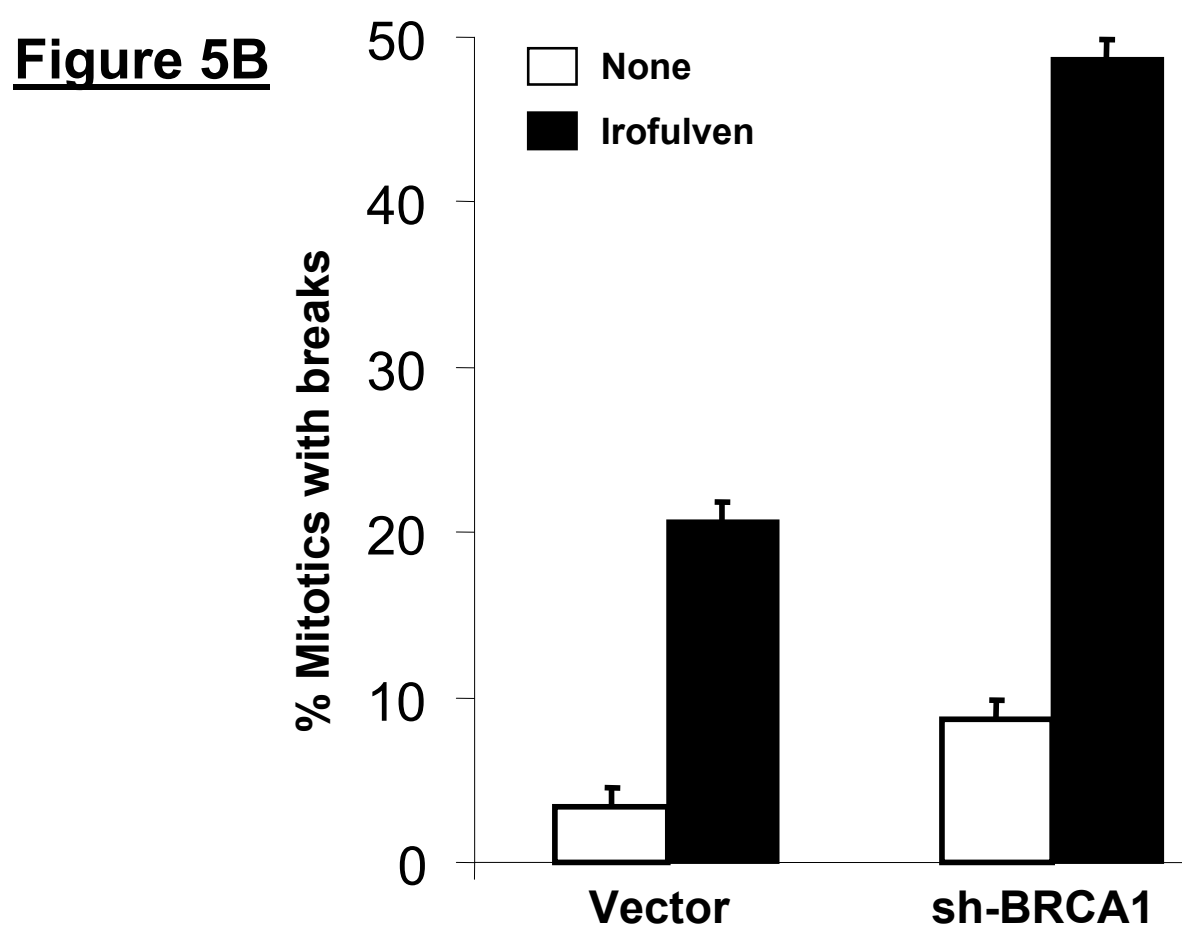


Figure 5C

\section{sh-BRCA1}
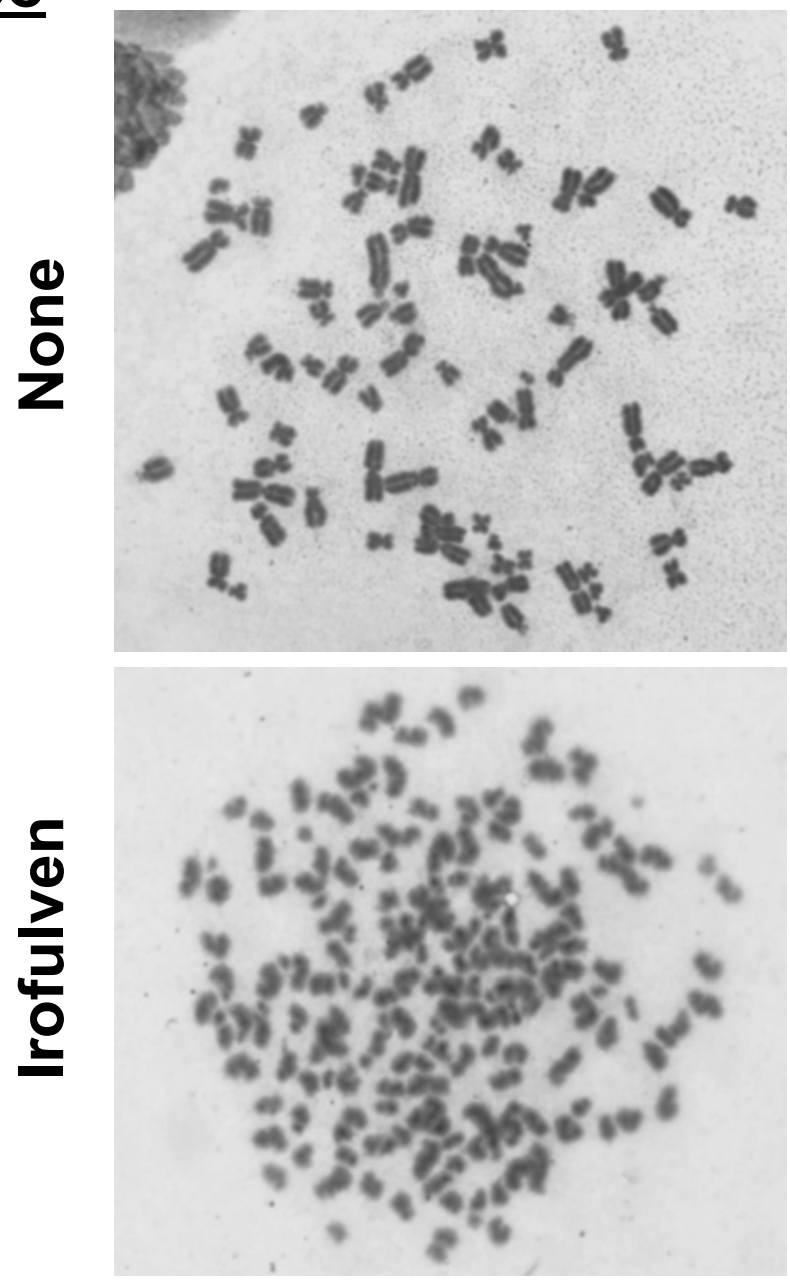
Figure 5D
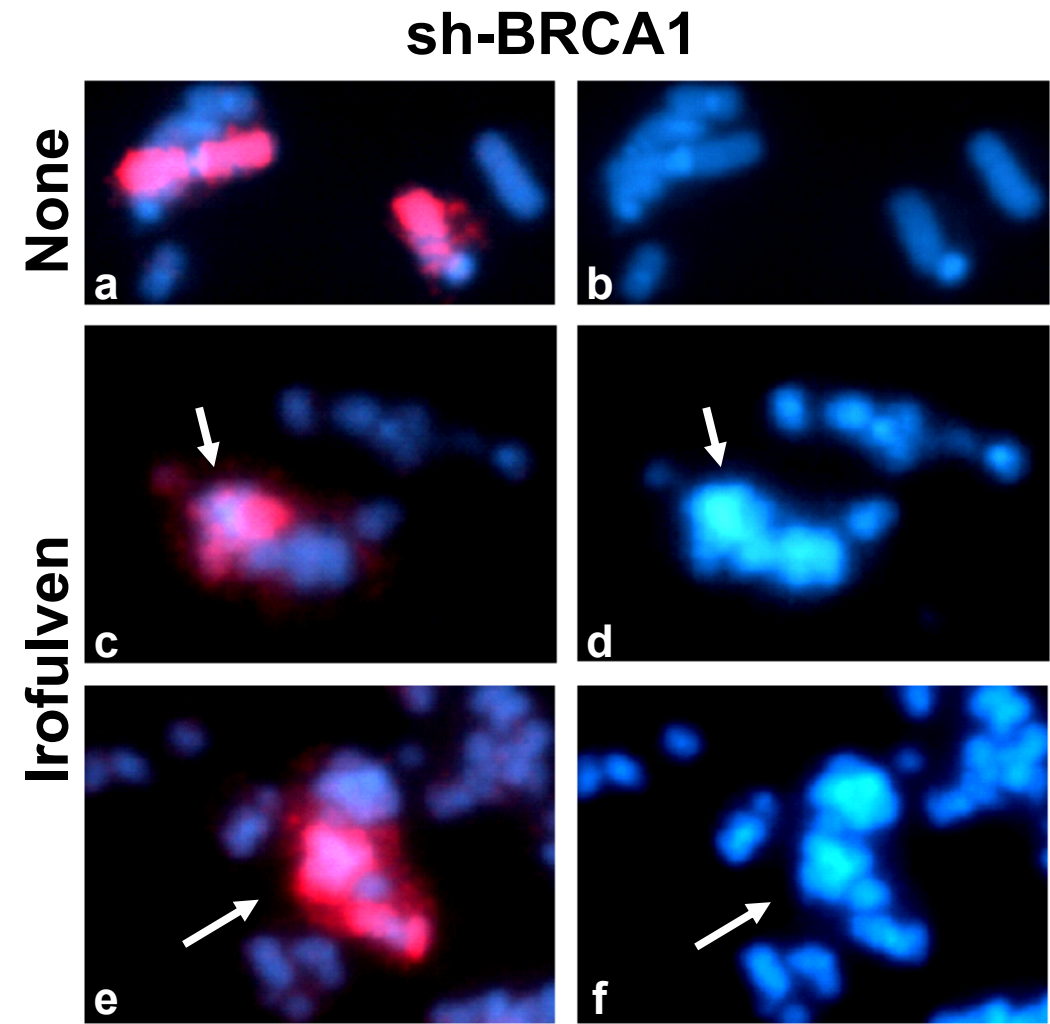
Figure 6. BRCA1 confers chemoresistance to irofulven.

Figure 6A

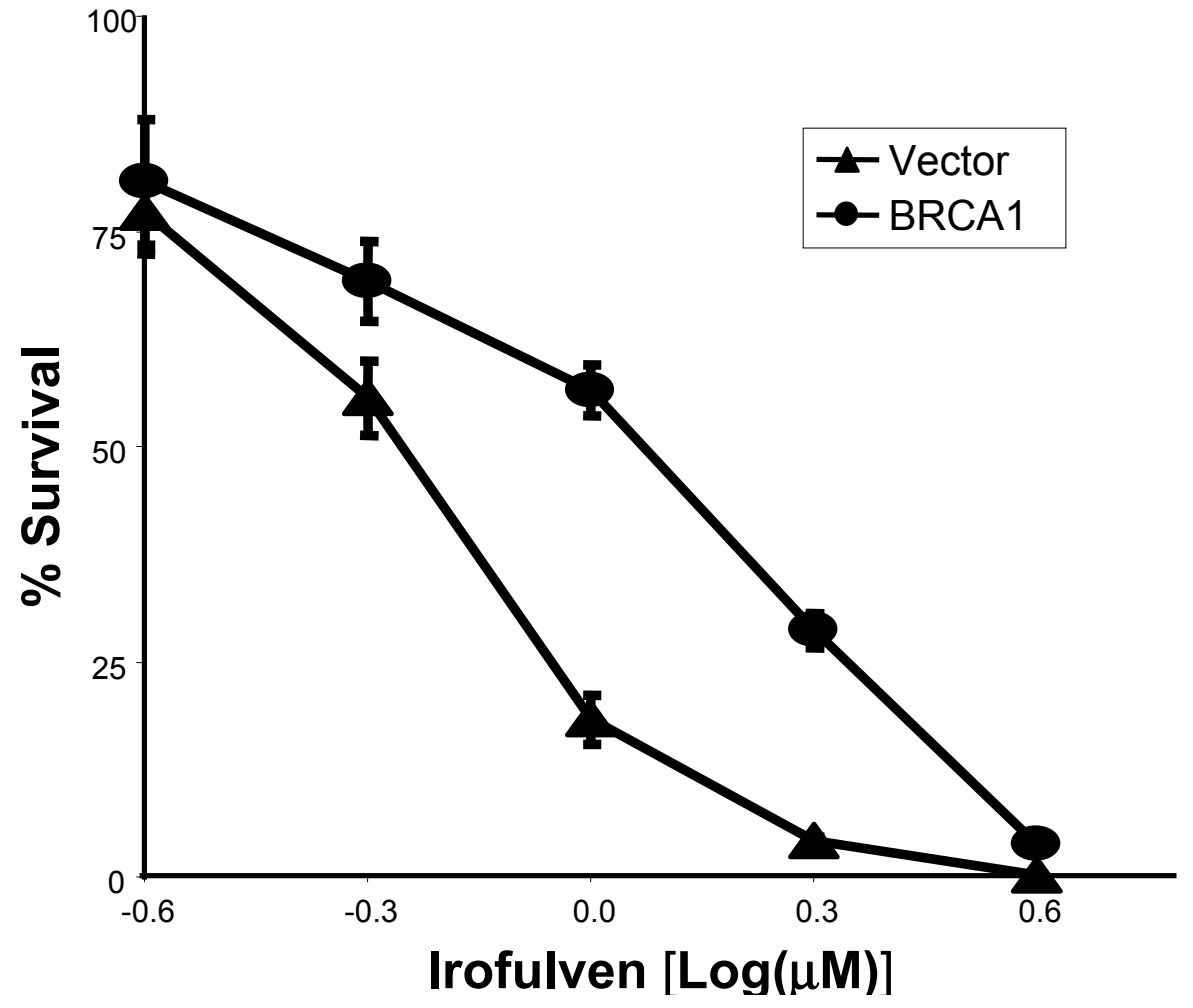

Figure 6B $\quad 100$

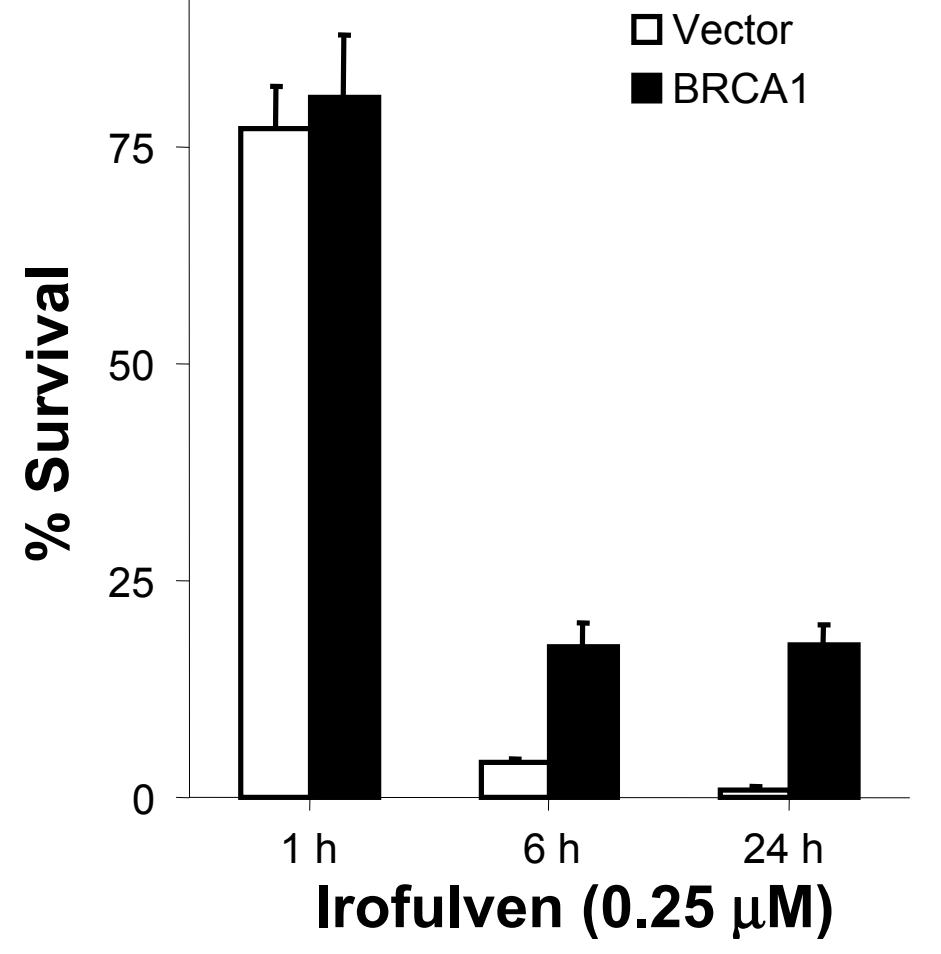


Figure 6C

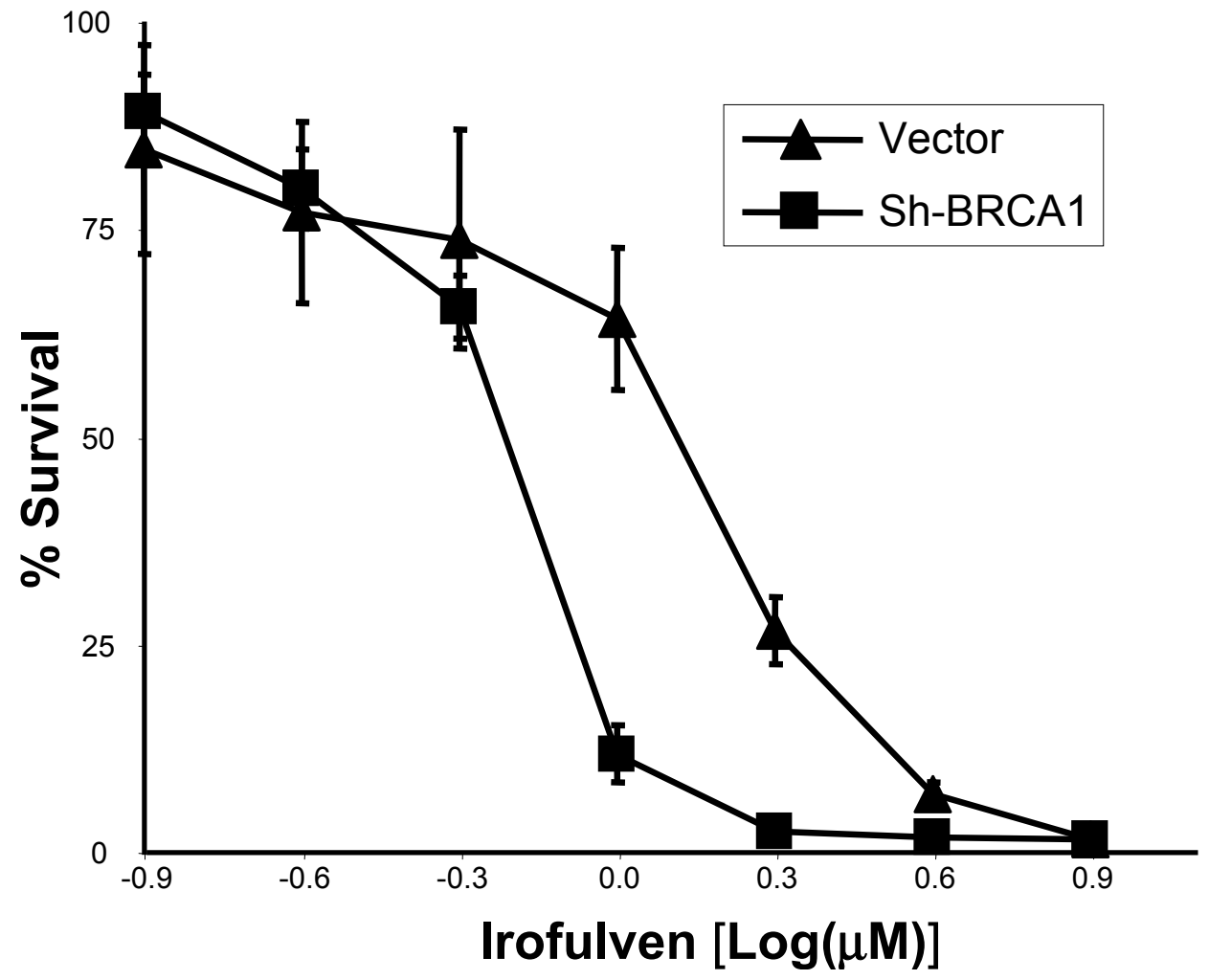




\section{Chapter III}

\section{Caspase-2 is involved in initiating irofulven-induced cell death}

Timothy Wiltshire ${ }^{1,2}$, Steven M. Frisch ${ }^{1,3}$, Eddie Reed ${ }^{1,2,4}$, and Weixin Wang ${ }^{1,2, *}$

${ }^{1}$ Mary Babb Randolph Cancer Center; ${ }^{2}$ Department of Microbiology, Immunology and

Cell Biology; and ${ }^{3}$ Department of Biochemistry, West Virginia University School of Medicine, Morgantown, WV 26506.

Keywords: Caspase-2, DNA damage, apoptosis, chemosensitivity, irofulven

${ }^{4}$ Present address: Centers for Disease Control and Prevention, Atlanta, Georgia. 


\begin{abstract}
Induction of apoptosis is a desirable trait for chemotherapeutic agents. Irofulven (6-hydroxymethylacylfulvene, MGI 114, NSC\#: 683863) is one of a new class of anticancer agents that are analogs of the mushroom derived illudin toxins. Preclinical studies and clinical trials have demonstrated that irofulven is effective against several tumor cell types. It has been shown that irofulven induces DNA damage, MAP kinase activation and apoptosis; and activates several proteins in the DNA damage response pathway, leading to cell cycle arrest and influencing chemosensitivity. In this study, we investigated the role of caspase-2 in initiating irofulven-induced apoptosis. We also examined the effect of irofulven-induced apoptosis on chemosensitivity. Using isogenic mouse embryonic fibroblasts proficient and deficient for caspase-2 and Apaf-1, we demonstrated that irofulven-induced cell death was mediated by the mitochondrial apoptotic pathway and that caspase- 2 was involved in initiating irofulven-induced apoptosis through the mitochondrial apoptotic pathway. Furthermore, we showed that caspase- 2 and the mitochondria-controlled apoptotic pathway enhanced chemosensitivity to irofulven.
\end{abstract}

Keywords: Caspase-2, DNA damage, apoptosis, chemosensitivity, irofulven 


\section{Introduction}

Caspase-dependent apoptosis is a form of programmed cell death controlled by a group of cysteine proteases known as caspases. It is characterized by cellular body shrinkage, plasma membrane blebbing, chromatin condensation and DNA fragmentation [1-3]. Both an intrinsic and extrinsic pathway of activation exists. The extrinsic or death receptor pathway is triggered by the activation of members of the death receptor family, including FasL/Fas, TNF/TNFR, TRAIL/DR4, and TRAIL/DR5. This leads to the activation of caspase- 8 and apoptosis. The intrinsic or mitochondria pathway is initiated by the change in mitochondrial membrane potential, which results in the release of cytochrome c, AIF, Smac/DIABLO and Omi/HtrA2 from the mitochondria [1-3]. Cytochrome c and Apaf-1 form a multimeric complex called the "apoptosome" in the presence of dATP/ATP, recruiting procaspase-9 and promoting its efficient activation [46]. Activated caspase-9 in turn cleaves and activates downstream effectors caspase-3, -6 and -7 resulting in apoptosis [1-3]. Caspase-2 responds predominantly to genotoxic stress via a poorly defined mechanism [1-3]. Some studies have reported the recruitment of procaspase-2 into a multimeric complex, which is sufficient to activate it in a manner independent of mitochondrial activity [7]; while other data reveals that caspase-2 is able to cause mitochondrial dysfunction leading to cytochrome c release, supporting a role for caspase-2 upstream of the mitochondria and a requirement for caspase-9 [8-16]. While no definitive answer currently exists, it is reported that caspase- 2 is important for signaling of apoptosis induced by DNA damage agents, such as cisplatin, etoposide and UV [12$16]$. 
Induction of apoptosis by chemotherapeutic agents remains a highly desirable and effective trait. There are several first line therapies, as well as ionizing radiation, that rely on their ability to damage DNA in order to kill cancerous cells. DNA damaging agents have been documented to be potent inducers of apoptosis [17]. While we know they are able to induce apoptosis, the exact mechanism of action of these anticancer agents remains poorly understood. Based on the damage inflicted, each agent activates a unique response. Therefore, it is important to understand what signals these agents use to activate apoptosis in order to determine which tumors may respond more robustly to specific chemotherapeutics. Irofulven (6-hydroxymethylacylfulvene, MGI 114, NSC\#: 683863 ) is one of a new class of anticancer agents that are analogs of mushroom-derived illudin toxins. Preclinical studies and clinical trials have shown that irofulven is effective against several tumor cell types [18-26]. While the exact mechanism of action of irofulven is not well understood, it has been shown that irofulven induces DNA damage, MAP kinase activation and apoptosis; and activates several proteins in the DNA damage response pathway, leading to cell cycle arrest and influencing chemosensitivity [27-35].

To further understand the mechanism of action of irofulven, we hypothesized that caspase-2 may play an important role in initiating irofulven-induced apoptosis. By using isogenic mouse embryonic fibroblasts proficient and deficient for caspase-2 and Apaf-1, we investigated the role that caspase-2 might play in irofulven-induced apoptosis. We observed that irofulven-induced cell death is mediated by the mitochondrial apoptotic pathway and that caspase- 2 is involved in initiating irofulven-induced apoptosis through the mitochondrial apoptotic pathway. Furthermore, we demonstrated that caspase- 2 and the mitochondria-controlled apoptotic pathway enhanced chemosensitivity to irofulven. 


\section{Materials and Methods}

Cell Culture - Mouse embryonic fibroblasts (MEFs) knocking-out Apaf-1 (generously provided by Prof. Tak Mak of the University of Toronto, Toronto, Canada) [36] and MEFs knocking-out caspase-2 (generously provided by Dr. Andreas Strasser of the Walter and Eliza Hall Institute of Medical Research, Melbourne, Australia) [37] were cultured in DMEM (Mediatech, Herndon, VA) supplemented with 10\% fetal bovine serum and penicillin/streptomycin in a $37^{\circ} \mathrm{C}$ incubator with $5 \% \mathrm{CO}_{2}$ atmosphere.

Western blotting - Western blot was performed as described previously [35]. Whole cell lysates $(100 \mu \mathrm{g})$ were separated by SDS-PAGE. Primary antibodies used were: rat antimouse caspase-2 (1:1000) (Alexis Biochemicals, San Diego, CA), rabbit anti-Apaf-1 (1:100; Santa Cruz Biotechnology, Santa Cruz, CA) and mouse anti-actin (1:5000; Sigma, St. Louis, MO). Secondary antibodies used were: sheep anti-mouse IgG-HRP, sheep anti-rabbit IgG-HRP (1:4,000; GE Healthcare, Piscataway, NJ) and goat anti-rat IgG-HRP (1:4000; Santa Cruz Biotechnology, Santa Cruz, CA). All dilutions were done as indicated in PBS containing 0.1\% Tween-20 and 5\% milk.

Apoptosis ELISA - Apoptosis ELISA was performed using the Cell Death Detection ELISA $^{\text {PLUS }}$ kit (Roche, Indianapolis, IN) according to manufacturer's instructions to detect cytoplasmic histone-associated DNA fragments (mono- and oligonucleosomes). Cells were plated in 6-well plates and allowed to attach overnight. Treatment with irofulven was done with various doses and exposure times as noted. After drug exposure, 
both the floating and adherent cells were collected and washed with ice-cold PBS. Cell pellets were then lysed in provided lysis buffer for $30 \mathrm{~min}$ at room temperature followed by $10 \mathrm{~min}$ of centrifugation at $1500 \mathrm{~g}$. The supernatant $(20 \mu \mathrm{l})$ was added to each well along with $80 \mu \mathrm{l}$ of immunoreagent consisting of anti-DNA and anti-POD antibodies. Microplate was covered and allowed to shake at $300 \mathrm{rpm}$ for two hours at room temperature. Following incubation, the wells were washed three times with incubation buffer and $100 \mu \mathrm{l}$ of ABTS solution was added. Microplate was allowed to shake at 250 rpm for 5-10 minutes and then read at $405 \mathrm{~nm}$ on a spectrophotometer (Molecular Devices, Sunnyvale, CA). Results are reported as fold change from untreated cells minus background and represent three independent experiments.

Propidium iodide staining and flow cytometry - Analysis of cells undergoing apoptosis was done using propidium iodide (PI) staining and flow cytometry. Cells were treated with irofulven and harvested by mechanical detachment. After washing in PBS, cell pellets were fixed in 70\% ethanol in PBS overnight. Fixed cells were washed twice in PBS and suspended in a final solution of PBS containing propidium iodide $(1 \mu \mathrm{g} / \mathrm{ml})$ and RNase A $(20 \mu \mathrm{g} / \mathrm{ml})$. Samples were incubated for $30 \mathrm{~min}$ at $4{ }^{\circ} \mathrm{C}$ in the dark followed by measurement of PI content (20,000 events each) using a FACSCalibur cytometer with CellquestPro software (BD Biosciences, San Jose, CA). The sub-G1 portion of the histogram was quantified as the relative percentage of apoptotic cells.

Chromogenic caspase activity assay - The chromogenic caspase substrates Ac-VDVADpNA, Ac-LEHD-pNA and Ac-DEVD-pNA (Anaspec, San Jose, CA) were used to 
measure caspase-2, caspase-9 and caspase-3 activities, respectively. After irofulven treatment, cells were collected and washed twice with ice-cold PBS and lysed in cell lysis buffer (10 mM HEPES, 2 mM EDTA, 0.1\% Igepal, 5 mM DTT, 1 mM PMSF, $10 \mu \mathrm{g} / \mathrm{ml}$ pepstatin A, $20 \mu \mathrm{g} / \mathrm{ml}$ leupeptin, $10 \mu \mathrm{g} / \mathrm{ml}$ aprotinin). Protein concentration of the supernatant was determined using the Bradford assay kit (BioRad, Hercules, CA). Protein extract $(50-100 \mu \mathrm{g})$ of each sample was added and adjusted to $100 \mu 1$ with cell lysis buffer. Substrate assay buffer (100 $\mu 1)(100 \mathrm{mM}$ HEPES, 20\% glycerol, 1 mM EDTA, $0.1 \%$ CHAPS and $10 \mathrm{mM}$ DTT $)$ containing specific caspase substrate $(300 \mu \mathrm{M})$ was added to each reaction and incubated at $37^{\circ} \mathrm{C}$ for $12-15$ hours. The generation of free pNA was measured by an UQuant spectrophotometer (Biotek, Winooski, VT) at $405 \mathrm{~nm}$. Fold increase on caspase activity was determined by subtracting background control from each sample and dividing by the un-treated control.

Clonogenic survival assay - To determine chemosensitivity, clonogenic survival assay was performed as previously described [35]. Cells were plated in 60-mm dishes overnight in complete medium and treated with different concentrations of irofulven for 24 hours followed by drug-free incubation for 7-10 days. Colonies were stained with PBS containing $0.04 \%$ crystal violet and $0.5 \%$ paraformaldehyde for $20 \mathrm{~min}$. Staining liquid was aspirated and colonies counted. Results are reported as treated divided by un-treated control and represent three individual experiments. 


\section{Results}

\section{Irofulven-induced cell death is mediated by the mitochondrial apoptotic pathway.}

Caspase-2 has been reported to initiate apoptosis induced by several stress stimuli, such as ceramide, cisplatin, etoposide, docetaxel or UV, through the mitochondriadependent or independent apoptotic pathways [7-16]. In this study, we attempted to determine whether caspase-2 is involved in initiating irofulven-induced apoptosis. We first examined the involvement of the mitochondrial apoptotic pathway in response to irofulven using mouse embryonic fibroblasts (MEFs) deficient or proficient for Apaf-1 [36]. Apaf-1 -/- MEFs are impaired for apoptosome formation and caspase-9 activation, and thus mitochondria-mediated apoptosis [4-6]. Western blot analysis confirmed the status of Apaf-1 protein in the previously described Apaf-1 knockout cells [36] (Fig. 1A). We then used an ELISA-based method detecting DNA fragmentation to measure the induction of apoptosis. Compared to their knockout counterparts, wild-type Apaf-1 cells underwent significantly more apoptosis when exposed to 5 or $10 \mu \mathrm{M}$ of irofulven for 24 hours $(p<0.001)$ (Fig. 1B).

To verify this phenomenon, we used propidium iodide (PI) staining and flow cytometry as an alternate method to measure the induction of apoptosis. The same set of isogenic Apaf-1 MEFs was treated with irofulven. Cells were stained with PI and the subG1 DNA content was measured by flow cytometry. Figure 1C depicts a set of representative histograms showing the cell cycle distribution before and after irofulven treatment. The relative amount of apoptosis was quantified as the M1 region which includes all cells with sub-G1 DNA content (Fig. 1C). Results indicated that wild-type 
Apaf-1 cells underwent much more apoptosis than their knockout counterparts after irofulven treatment (Fig. 1D). From these data, we concluded that the mitochondrial apoptotic pathway plays an important role in the induction of apoptosis upon irofulven treatment.

\section{Caspase-2 is involved in initiating irofulven-induced apoptosis through the mitochondrial apoptosis pathway.}

To determine the role that caspase- 2 may play in response to irofulven treatment, we used isogenic caspase-2 -/- and +/+ MEFs. The status of caspase-2 expression in these cells was confirmed by western blot analysis (Fig. 2A). The apoptosis induction was measured by using DNA fragmentation ELISA. Results indicated that the caspase- $2+/+$ cells underwent several-fold more apoptosis when compared to the caspase-2 -/- cells after 10 or $20 \mu \mathrm{M}$ of irofulven treatment ( $p<0.001$ or $p<0.01$, respectively) (Fig. $2 \mathrm{~B}$ ).

To confirm this finding, PI staining and flow cytometry were performed in these cells. Many more cells containing sub-G1 DNA content were observed in caspase- $2+/+$ cells (Fig. 2C) and the results of triplicate experiments were graphed in figure 2D. These results demonstrated that there was a dramatic increase in apoptosis in caspase $-2+/+$ cells compared to caspase 2 -/- cells after irofulven treatment. This strongly indicates a role for caspase-2 in irofulven-induced apoptosis.

To further verify caspase activation and determine whether caspase- 2 is involved in initiating apoptosis through the mitochondrial pathway, we determined caspase enzyme activities in Apaf-1 and caspase-2 MEFs by using caspase substrates conjugated to p-nitroanilide (pNA). These caspase substrates emit measurable chromogenic signals 
upon the cleavage of pNA. When the caspase-9 substrate, Ac-LEHD-pNA, was incubated with whole cell lysates of Apaf-1 -/- and Apaf- $1+/+$ cells after irofulven treatment, it was shown that caspase-9 was preferentially activated in wild-type Apaf-1 cells $(p<0.001)$ (Fig. 3A). The overall level of apoptosis was also surveyed using the caspase-3 substrate, Ac-DEVD-pNA. The results indicated that, consistent with previous observations, more induction of apoptosis, as measured by caspase-3 activity, occurred in the Apaf- $1+/+$ cells after irofulven treatment $(p<0.001)$ (Fig. 3B).

Next, we performed this caspase activity assay in isogenic caspase- $2-/$ - and caspase- $2+/+$ cells to determine the dependence of specific substrate cleavage on caspase- 2 activity after irofulven treatment. As expected, when the caspase- 2 substrate, Ac-VDVAD-pNA, was incubated with whole cell lysates of caspase-2 -/- and caspase-2 $+/+$ cells, it was demonstrated that caspase- 2 was preferentially activated in the caspase- 2 $+/+$ cells after irofulven treatment $(p<0.001)$ (Fig. 4A). When the caspase-9 substrate, Ac-LEHD-pNA, was used in both cells, it was found that caspase-9 was preferentially activated in the caspase- $2+/+$ cells $(p<0.001)$ (Fig. 4B), indicating that caspase-9 activation is dependent on caspase-2. In addition, caspase- 3 activity was also measured in these cells with the caspase-3 substrate, Ac-DEVD-pNA. It was shown that more apoptosis occurred in caspase- $2+/+$ cells than in caspase- $2-/-$ cells $(p<0.001)$ (Fig. 4C). Taken together, these results indicate that caspase-2 activation is involved in initiating irofulven-induced apoptosis through the mitochondria-mediated apoptotic pathway.

\section{Caspase-2 initiated mitochondrial apoptosis pathway enhances chemosensitivity to}

\section{irofulven.}


Activation of proteins that make up the apoptotic pathway is one way of determining the rate of cell death after treatment with a chemotherapeutic agent. Clonogenic survival assay is a more reliable method to determine the consequence of cell death in drug treated cells and thus chemosensitivity $[38,39]$. To assess whether caspase2 initiated mitochondrial apoptotic pathway renders cells more sensitive to irofulven, we performed clonogenic survival assay in isogenic Apaf-1 and caspase-2 MEFs. These cells were treated with 2 or $4 \mu \mathrm{M}$ of irofulven and the results demonstrated that both caspase- 2 and Apaf-1 render cells more sensitive to irofulven ( $p<0.05$ or $p<0.005$, respectively) (Fig. 5, A and B). These results indicated that caspase-2 initiated mitochondrial apoptotic pathway enhanced chemosensitivity to irofulven. 


\section{Discussion}

In this study, we showed that irofulven-induced cell death is initiated by caspase2 and is subsequently mediated through activation of the mitochondrial apoptotic pathway. We also demonstrated that caspase-2 and the mitochondria-controlled apoptotic pathway enhance chemosensitivity to irofulven. The mitochondrial pathway is a key component in inducing apoptosis in response to ionizing radiation and several chemotherapeutic agents $[1-3,36,40,41]$. It is still unclear whether caspase-9 acts alone or in conjunction with other apoptotic pathways. Caspase- 2 represents an initiator of apoptosis with a role that is not yet well-defined. Several reports have indicated that, in response to DNA damaging agents, caspase-2 initiates apoptosis upstream of mitochondrial cytochrome c release and caspase-9 activation leading to downstream effector caspase activation [8-16]. Another report has suggested that caspase-2 is able to form large complexes that are sufficient for activation of downstream effector caspases independent of cytochrome $\mathrm{c}$ and Apaf-1 [7]. This line of evidence supports a mechanism in which caspase-2 alone is able to induce apoptosis, whereas subsequent downstream cytochrome c release and caspase- 9 activation act as an independent amplification loop [7]. In this study, the caspase activity assay with specific caspase substrates clearly indicates that caspase- 2 is involved in initiating irofulven-induced apoptosis through the mitochondria-dependent mechanism.

How caspase- 2 is activated by various stress stimuli is still poorly understood. It has been reported that caspase-2 activation occurs by forming complex with PIDD (p53induced protein with a death domain) and the adapter protein RAIDD (RIP(ribosome- 
inactivating protein)-associated ICH-1/CED-homologous protein with death domain). Increased PIDD expression may result in caspase-2 activation as a means to regulate apoptosis $[16,42]$. However, a recent study indicated that caspase-2 activation is independent of p53 in response to docetaxel-induced apoptosis [11]. We have observed that irofulven induces p53 accumulation and activation [31,34]. However, we and others have conducted studies in tumor cell lines with wild-type, mutant or null p53; and demonstrated that the cytotoxicity induced by irofulven is independent of p53 status [ 34 , 43]. Therefore, caspase- 2 initiated apoptosis induced by irofulven may not be regulated by $\mathrm{p} 53$. Additional work is required to identify the potential proteins triggering caspase- 2 activation in response to irofulven.

The short term measurements of apoptosis may only determine a difference in the kinetics of cell death but not overall survival $[38,39]$. It is also possible that, in the overall scheme of cell death, one pathway may be able to compensate for the loss of another. We performed clonogenic survival assay to address such a situation and verified that differences in activation of apoptosis were actually translated into differences in overall survival. Both Apaf-1 and caspase-2 wild-type cells showed an increase in chemosensitivity to irofulven.

It should also be mentioned that caspase dependent apoptosis may not be the only mechanism by which irofulven is able to kill cells. Previous work has identified a role for caspase independent cell death in irofulven induced killing [44]. While the differences we report may seem minor, it must be noted that while we seek to determine the involvement of individual pathways, the other cell death pathways are still intact in these experiments. This would result in an overall decrease in the difference between the cell lines because 
we are only measuring one aspect of the response that may only represent a fraction of the total response. One cell death pathway could easily compensate for the loss of another thereby masking the response and diminishing the effect of the loss of a single gene. There is also the fact that our MEFs contain intact cell cycle checkpoints and DNA repair proteins which would render the cells more resistant to irofulven induced cell death [31, 33-35]. Also contributing to a minimal difference in chemosensitivity is the fact that caspase independent cell death may also contribute to overall killing and may further mask the response. Therefore, while the differences may seem minor, they are statistically significant and highly reproducible.

Another important point to discuss is the measurement of caspase activity using the substrate assay. Substrates that we use in these experiments that we call specific are not exclusive to the specific caspases, but are most strongly activated both those caspases. Strong evidence indicates that while they are preferentially activated by specific caspases, most substrates are also weakly activated by other caspase as well [45]. Such is the case for many caspase substrates that have overlapping specificities. This concept explains why we see minimal activation of what we call specific substrates even when we use knockout cells for that particular substrate. The important point in evaluating these assays is the difference between the isogenic cell lines all of which were significant in our experiments.

In summary, by using isogenic mouse embryonic fibroblasts proficient and deficient for caspase-2 and Apaf-1, we observed that irofulven-induced cell death is mediated by the mitochondrial apoptotic pathway and that caspase-2 is involved in initiating irofulven-induced apoptosis through the mitochondrial apoptotic pathway. We 
also demonstrated that caspase- 2 and the mitochondria-controlled apoptosis enhance chemosensitivity to irofulven. These findings will enhance our understanding of the mechanisms of apoptosis induction by irofulven. They will also provide insight for future studies to elucidate proteins that cause caspase-2 activation and the link between DNA damage signaling and caspase-2 initiated apoptosis induced by irofulven. 


\section{Acknowledgments}

We would like to thank Prof. Tak Mak (University of Toronto, Toronto, Canada) for generously providing Apaf-1 knock-out MEFs and Dr. Andreas Strasser (The Walter and

Eliza Hall Institute of Medical Research, Melbourne, Australia) for generously providing caspase 2 knock-out MEFs. This work was supported in part by a grant from the National Cancer Institute (5R03CA107979) and funding from the Fannie Rippel Foundation (to W. W.). 


\section{References}

1. Thornberry NA and Lazebnik Y, Caspases: enemies within. Science 281(5381): 1312-6, 1998.

2. Green DR, Apoptotic pathways: paper wraps stone blunts scissors. Cell 102(1): 1$4,2000$.

3. Roth W and Reed JC, Apoptosis and cancer: when BAX is TRAILing away. Nat Med 8(3): 216-8, 2002.

4. Rodriguez J and Lazebnik Y, Caspase-9 and APAF-1 form an active holoenzyme. Genes Dev 13(24): 3179-84, 1999.

5. Zou H, Li Y, Liu X and Wang X, An APAF-1.cytochrome c multimeric complex is a functional apoptosome that activates procaspase-9. J Biol Chem 274(17): 11549-56, 1999.

6. Acehan D, Jiang X, Morgan DG, Heuser JE, Wang X and Akey CW, Threedimensional structure of the apoptosome: implications for assembly, procaspase-9 binding, and activation. Mol Cell 9(2): 423-32, 2002.

7. Read SH, Baliga BC, Ekert PG, Vaux DL and Kumar S, A novel Apaf-1independent putative caspase-2 activation complex. J Cell Biol 159(5): 739-45, 2002. 8. Paroni G, Henderson C, Schneider C and Brancolini C, Caspase-2-induced apoptosis is dependent on caspase-9, but its processing during UV- or tumor necrosis factor-dependent cell death requires caspase-3. J Biol Chem 276(24): 21907-15, 2001. 
9. Guo Y, Srinivasula SM, Druilhe A, Fernandes-Alnemri T and Alnemri ES, Caspase-2 induces apoptosis by releasing proapoptotic proteins from mitochondria. $J$ Biol Chem 277(16): 13430-7, 2002.

10. Robertson JD, Enoksson M, Suomela M, Zhivotovsky B and Orrenius S, Caspase2 acts upstream of mitochondria to promote cytochrome c release during etoposideinduced apoptosis. J Biol Chem 277(33): 29803-9, 2002.

11. Mhaidat NM, Wang Y, Kiejda KA, Zhang XD and Hersey P, Docetaxel-induced apoptosis in melanoma cells is dependent on activation of caspase-2. Mol Cancer Ther 6(2): 752-61, 2007.

12. Lassus P, Opitz-Araya X and Lazebnik Y, Requirement for caspase-2 in stressinduced apoptosis before mitochondrial permeabilization. Science 297(5585): 1352-4, 2002.

13. Zhivotovsky B and Orrenius S, Caspase-2 function in response to DNA damage. Biochem Biophys Res Commun 331(3): 859-67, 2005.

14. Lin CF, Chen CL, Chang WT, Jan MS, Hsu LJ, Wu RH, Fang YT, Tang MJ, Chang WC and Lin YS, Bcl-2 Rescues Ceramide- and Etoposide-induced Mitochondrial Apoptosis through Blockage of Caspase-2 Activation. J Biol Chem 280(25): 23758-65, 2005.

15. Wagner KW, Engels IH and Deveraux QL, Caspase-2 can function upstream of bid cleavage in the TRAIL apoptosis pathway. J Biol Chem 279(33): 35047-52, 2004. 16. Tinel A and Tschopp J, The PIDDosome, a protein complex implicated in activation of caspase-2 in response to genotoxic stress. Science 304(5672): 843-6, 2004. 
17. Norbury CJ and Zhivotovsky B, DNA damage-induced apoptosis. Oncogene 23(16): 2797-808, 2004.

18. MacDonald JR, Muscoplat CC, Dexter DL, Mangold GL, Chen SF, Kelner MJ, McMorris TC and Von Hoff DD, Preclinical antitumor activity of 6hydroxymethylacylfulvene, a semisynthetic derivative of the mushroom toxin illudin S. Cancer Res 57(2): 279-83, 1997.

19. Sato Y, Kashimoto S, MacDonald JR and Nakano K, In vivo antitumour efficacy of MGI-114 (6-hydroxymethylacylfulvene, HMAF) in various human tumour xenograft models including several lung and gastric tumours. Eur J Cancer 37(11): 1419-28, 2001. 20. Friedman HS, Keir ST, Houghton PJ, Lawless AA, Bigner DD and Waters SJ, Activity of irofulven (6-hydroxymethylacylfulvene) in the treatment of glioblastoma multiforme-derived xenografts in athymic mice. Cancer Chemother Pharmacol 48(5): 413-6, 2001.

21. Hidalgo M, Izbicka E, Eckhardt SG, MacDonald JR, Cerna C, Gomez L, Rowinsky EK, Weitman SD and Von Hoff DD, Antitumor activity of MGI 114 (6hydroxymethylacylfulvene, HMAF), a semisynthetic derivative of illudin S, against adult and pediatric human tumor colony-forming units. Anticancer Drugs 10(9): 837-44, 1999. 22. Britten CD, Hilsenbeck SG, Eckhardt SG, Marty J, Mangold G, MacDonald JR, Rowinsky EK, Von Hoff DD and Weitman S, Enhanced antitumor activity of 6hydroxymethylacylfulvene in combination with irinotecan and 5-fluorouracil in the HT29 human colon tumor xenograft model. Cancer Res 59(5): 1049-53, 1999.

23. Murgo A, Cannon DJ, Blatner G and Cheson BD, Clinical trials referral resource. Clinical trials of MGI-114. Oncology (Huntingt) 13(2): 233, 237-8, 1999. 
24. Kelner MJ, McMorris TC, Rojas RJ, Trani NA, Velasco TR, Estes LA and Suthipinijtham P, Enhanced antitumor activity of irofulven in combination with antimitotic agents. Invest New Drugs 20(3): 271-9, 2002.

25. Senzer N, Arsenau J, Richards D, Berman B, MacDonald JR and Smith S, Irofulven demonstrates clinical activity against metastatic hormone-refractory prostate cancer in a phase 2 single-agent trial. Am J Clin Oncol 28(1): 36-42, 2005.

26. Woo MH, Peterson JK, Billups C, Liang H, Bjornsti MA and Houghton PJ, Enhanced antitumor activity of irofulven in combination with irinotecan in pediatric solid tumor xenograft models. Cancer Chemother Pharmacol 55(5): 411-9, 2005.

27. Kelner MJ, McMorris TC, Estes L, Starr RJ, Rutherford M, Montoya M, Samson KM and Taetle R, Efficacy of Acylfulvene Illudin analogues against a metastatic lung carcinoma MV522 xenograft nonresponsive to traditional anticancer agents: retention of activity against various mdr phenotypes and unusual cytotoxicity against ERCC2 and ERCC3 DNA helicase-deficient cells. Cancer Res 55(21): 4936-40, 1995.

28. Wang W, Waters SJ, MacDonald JR, Von Hoff DD, Strodel WE and Miller AR, Irofulven (6-hydroxymethylacylfulvene, MGI 114) induces caspase 8 and 9-mediated apoptosis in human pancreatic adenocarcinoma cells. Anticancer Res 21(3B): 1789-94, 2001.

29. Wang W, Waters SJ, MacDonald JR, Roth C, Shentu S, Freeman J, Von Hoff DD and Miller AR, Irofulven (6-hydroxymethylacylfulvene, MGI 114)-induced apoptosis in human pancreatic cancer cells is mediated by ERK and JNK kinases. Anticancer Res 22(2A): 559-64, 2002. 
30. Kelner MJ, McMorris TC, Estes L, Rutherford M, Montoya M, Goldstein J, Samson K, Starr R and Taetle R, Characterization of illudin S sensitivity in DNA repairdeficient Chinese hamster cells. Unusually high sensitivity of ERCC2 and ERCC3 DNA helicase-deficient mutants in comparison to other chemotherapeutic agents. Biochem Pharmacol 48(2): 403-9, 1994.

31. Wang J, Wiltshire T, Wang Y, Mikell C, Burks J, Cunningham C, Van Laar ES, Waters SJ, Reed E and Wang W, ATM-dependent CHK2 Activation Induced by Anticancer Agent, Irofulven. J Biol Chem 279(38): 39584-92, 2004.

32. Jaspers NG, Raams A, Kelner MJ, Ng JM, Yamashita YM, Takeda S, McMorris TC and Hoeijmakers JH, Anti-tumour compounds illudin S and Irofulven induce DNA lesions ignored by global repair and exclusively processed by transcription- and replication-coupled repair pathways. DNA Repair (Amst) 1(12): 1027-38, 2002.

33. Wang Y, Wiltshire T, Senft J, Wenger SL, Reed E and Wang W, Fanconi anemia D2 protein confers chemoresistance in response to the anticancer agent, irofulven. Mol Cancer Ther 5(12): 3153-61, 2006.

34. Wang Y, Wiltshire T, Senft J, Reed E and Wang W, Irofulven induces replication-dependent CHK2 activation related to p53 status. Biochem Pharmacol 73(4): 469-80, 2007.

35. Wiltshire T, Senft J, Wang Y, Konat GW, Wenger SL, Reed E and Wang W, BRCA1 contributes to cell cycle arrest and chemoresistance in response to the anticancer agent irofulven. Mol Pharmacol 71(4): 1051-60, 2007. 
36. Yoshida H, Kong YY, Yoshida R, Elia AJ, Hakem A, Hakem R, Penninger JM and Mak TW, Apafl is required for mitochondrial pathways of apoptosis and brain development. Cell 94(6): 739-50, 1998.

37. O'Reilly LA, Ekert P, Harvey N, Marsden V, Cullen L, Vaux DL, Hacker G, Magnusson C, Pakusch M, Cecconi F, Kuida K, Strasser A, Huang DC and Kumar S, Caspase-2 is not required for thymocyte or neuronal apoptosis even though cleavage of caspase-2 is dependent on both Apaf-1 and caspase-9. Cell Death Differ 9(8): 832-41, 2002.

38. Brown JM and Attardi LD, The role of apoptosis in cancer development and treatment response. Nat Rev Cancer 5(3): 231-7, 2005.

39. Brown JM and Wouters BG, Apoptosis, p53, and tumor cell sensitivity to anticancer agents. Cancer Res 59(7): 1391-9, 1999.

40. Hakem R, Hakem A, Duncan GS, Henderson JT, Woo M, Soengas MS, Elia A, de la Pompa JL, Kagi D, Khoo W, Potter J, Yoshida R, Kaufman SA, Lowe SW, Penninger JM and Mak TW, Differential requirement for caspase 9 in apoptotic pathways in vivo. Cell 94(3): 339-52, 1998.

41. Soengas MS, Alarcon RM, Yoshida H, Giaccia AJ, Hakem R, Mak TW and Lowe SW, Apaf-1 and caspase-9 in p53-dependent apoptosis and tumor inhibition. Science 284(5411): 156-9, 1999.

42. Berube C, Boucher LM, Ma W, Wakeham A, Salmena L, Hakem R, Yeh WC, Mak TW and Benchimol S, Apoptosis caused by p53-induced protein with death domain (PIDD) depends on the death adapter protein RAIDD. Proc Natl Acad Sci U S A 102(40): 14314-20, 2005. 
43. Izbicka E, Davidson K, Lawrence R, Cote R, MacDonald JR and Von Hoff DD, Cytotoxic effects of MGI 114 are independent of tumor p53 or p21 expression. Anticancer Res 19(2A): 1299-307, 1999.

44. Liang H, Salinas RA, Leal BZ, Kosakowska-Cholody T, Michejda CJ, Waters SJ, Herman TS, Woynarowski JM and Woynarowska BA, Caspase-mediated apoptosis and caspase-independent cell death induced by irofulven in prostate cancer cells. Mol Cancer Ther 3(11): 1385-96, 2004.

45. Talanian RV, Quinlan C, Trautz S, Hackett MC, Mankovich JA, Banach D, Ghayur T, Brady KD and Wong WW, Substrate specificities of caspase family proteases. J Biol Chem 272(15): 9677-82, 1997. 


\section{Footnotes}

${ }^{1}$ This work was supported in part by a grant from the National Cancer Institute (5R03CA107979) and funding from the Fannie Rippel Foundation (to W. W.).

${ }^{2}$ Present address: Centers for Disease Control and Prevention, Atlanta, Georgia. 


\section{Figure Legends}

Figure 1. Irofulven-induced cell death is mediated by the mitochondrial apoptosis pathway. A, western blot analysis was performed to verify the Apaf-1 expression status in Apaf-1 -/- and Apaf-1 +/+ MEFs. Actin was blotted as the loading control. B, induction of apoptosis was determined by apoptosis ELISA measuring DNA fragmentation. Apaf-1 -/- and Apaf-1 +/+ cells were treated with 1, 5 and $10 \mu \mathrm{M}$ of irofulven for 24 hours. The relative apoptosis was calculated as fold change from untreated cells minus background. The mean and standard deviation of triplicate experiments are shown. The statistical significance was analyzed by Student's $t$-test and marked as $*(p<0.001)$. C, quantification of apoptosis by propidium iodide (PI) staining and flow cytometry. Apaf-1 -/- and Apaf-1 +/+ cells were treated with 10 and $20 \mu \mathrm{M}$ of irofulven for 16 hours. A representative set of histograms from one experiment is shown. Apoptotic cells with sub-G1 DNA content is represented by the M1 region. D, graphical representation of the mean and standard deviation of triplicate experiments of PI staining and flow cytometry analysis.

Figure 2. Caspase-2 is involved in initiating irofulven-induced apoptosis. A, caspase-2 protein expression in caspase-2 -/- and caspase- $2+/+$ cells was verified by western blot. Actin was blotted as the loading control. B, induction of apoptosis was measured by apoptosis ELISA. Caspase-2 -/- and caspase-2 +/+ cells were treated for 24 hours with 10 and $20 \mu \mathrm{M}$ of irofulven. The relative apoptosis was calculated as fold change from untreated cells minus background. The mean and standard deviation of triplicate 
experiments are shown. The statistical significance was analyzed by Student's $t$-test and marked as $*(p<0.001)$ and $* *(p<0.01)$. C, quantification of apoptosis by PI staining and flow cytometry. Caspase-2 -/- and caspase- $2+/+$ cells were treated with 10 and $20 \mu \mathrm{M}$ of irofulven for 16 hours. A representative set of histograms from one experiment is shown. Apoptotic cells with sub-G1 DNA content is represented by the M1 region. D, diagram showing the mean and standard deviation of sub-G1 cell population from triplicate experiments of PI staining and flow cytometry analysis.

Figure 3. Irofulven activates caspase-9 of the mitochondrial apoptosis pathway. Chromogenic caspase activity assay was performed with whole cell lysates of Apaf-1 -/and Apaf $-1+/+$ cells treated with 10 and $20 \mu \mathrm{M}$ of irofulven for 18 hours. The mean and standard deviation of triplicate experiments are shown. The statistical significance was analyzed by Student's $t$-test and marked as * $(p<0.001)$. A, caspase- 9 activity was measured using Ac-LEHD-pNA as the substrate. B, the overall level of apoptosis was determined by measuring caspase-3 activity with Ac-DEVD-pNA as the substrate.

Figure 4. Caspase-2 initiates irofulven-induced apoptosis through the mitochondrial apoptosis pathway. Chromogenic caspase activity assay was performed with whole cell lysates of caspase-2 -/- and caspase- $2+/+$ cells treated with 10 and $20 \mu \mathrm{M}$ of irofulven for 18 hours. The mean and standard deviation of triplicate experiments are shown. The statistical significance was analyzed by Student's $t$-test and marked as * $(p<0.001)$. A, caspase-2 activity was measured using Ac-VDVAD-pNA as the substrate. B, caspase-9 activity was measured using Ac-LEHD-pNA as the substrate. C, the overall level of 
apoptosis was determined by measuring caspase-3 activity with Ac-DEVD-pNA as the substrate.

Figure 5. Caspase-2 initiated mitochondrial apoptosis pathway enhances chemosensitivity to irofulven. A and $\mathbf{B}$, clonogenic survival assay was performed in isogenic caspase-2 (A) and Apaf-1 (B) MEFs treated with 2 and $4 \mu \mathrm{M}$ of irofulven for 24 hours. The mean and standard deviation of triplicate experiments are presented. The statistical significance was analyzed by Student's $t$-test and marked as * $(p<0.05)$ and ** $(p<0.005)$ 
Figure 1. Irofulven-induced cell death is mediated by the mitochondrial apoptosis pathway.

Figure 1A $\frac{\text { Apaf-1 MEF }}{-/-\quad+/+}$

\section{Apaf-1}

\section{Actin}

Figure 1B

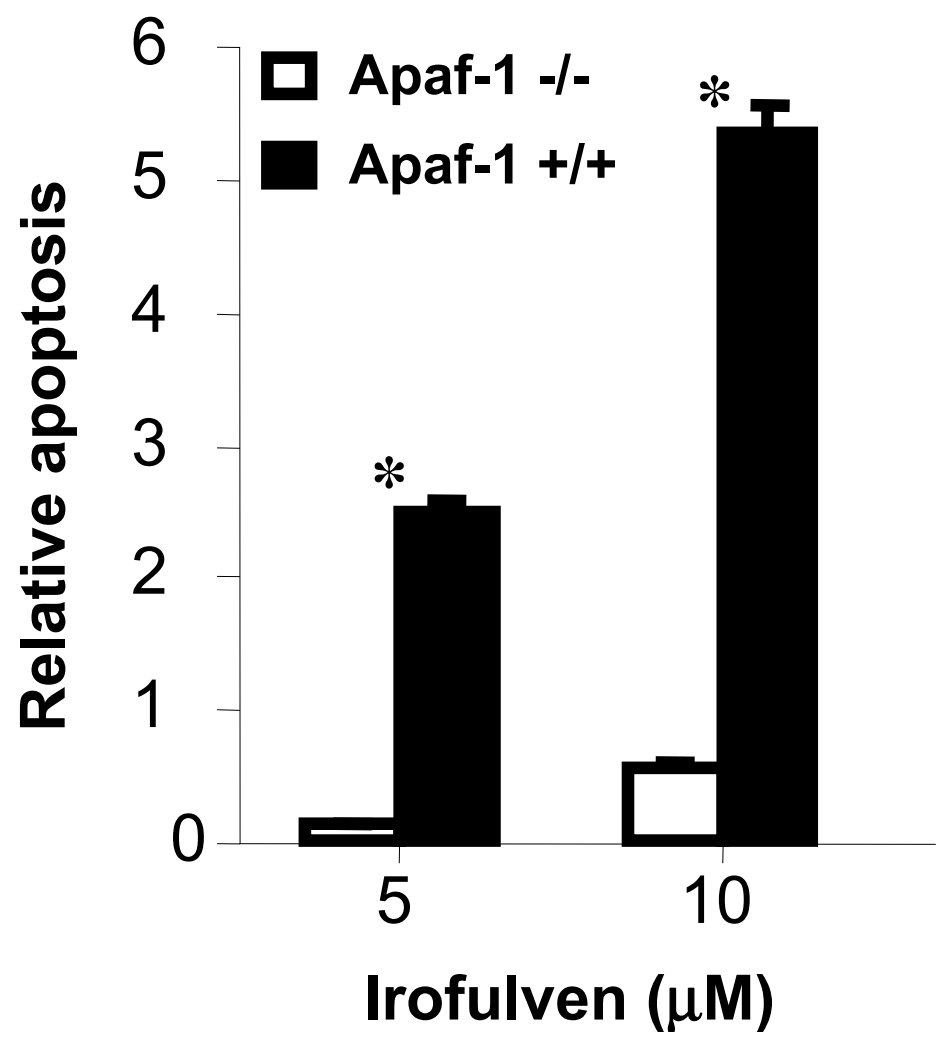


Figure 1C

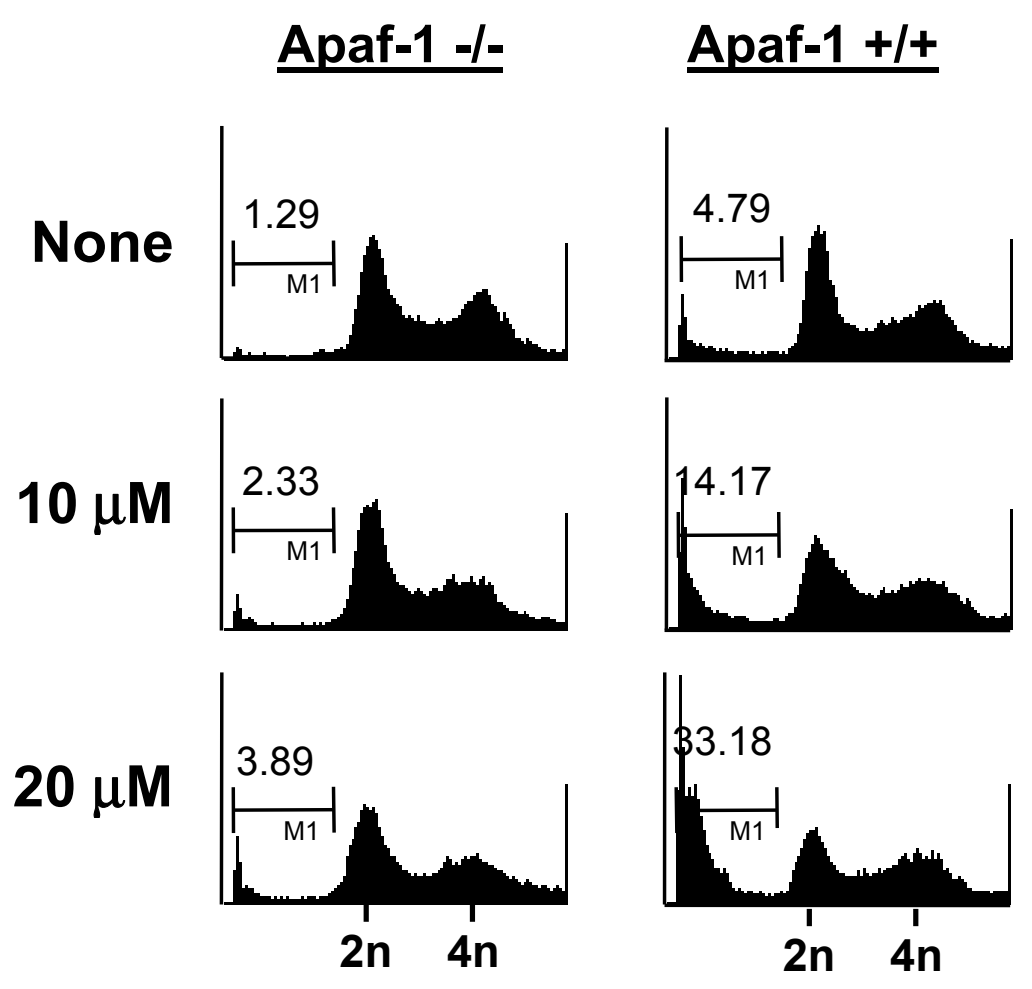

Figure 1D

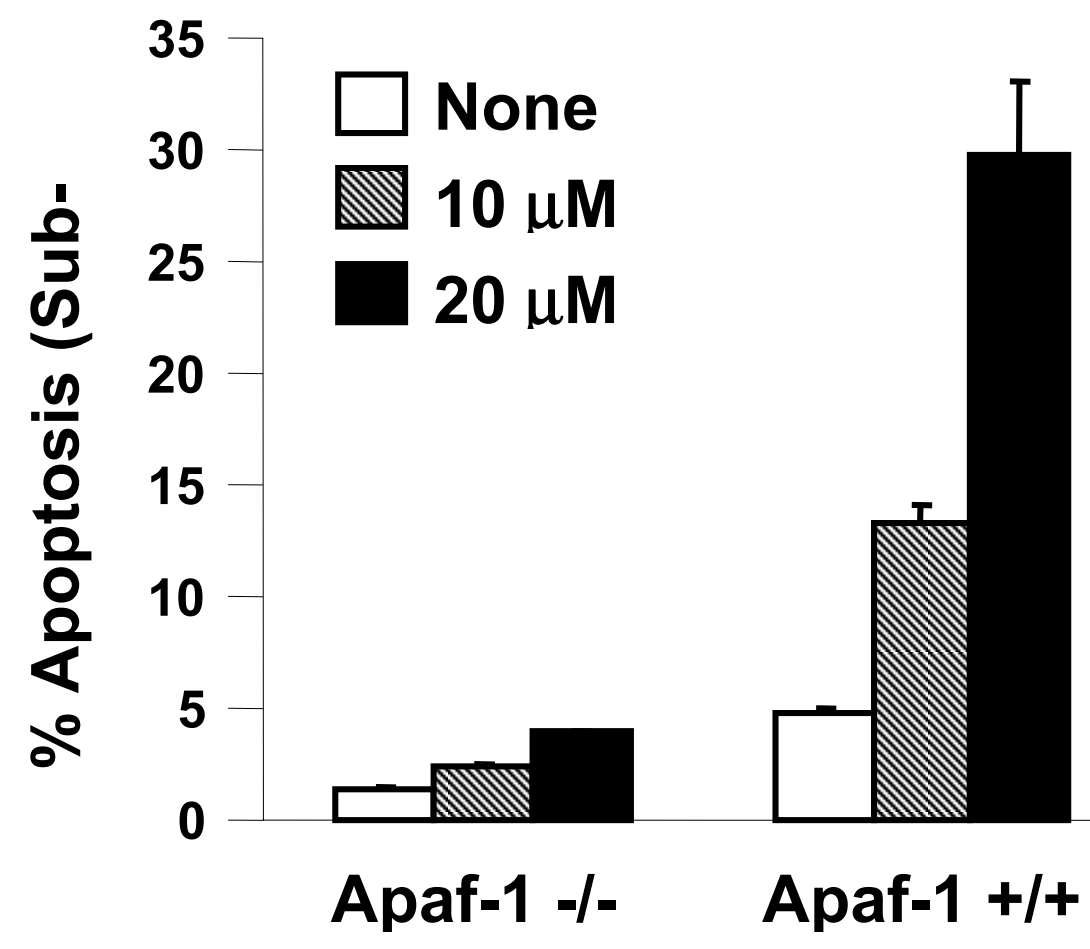


Figure 2. Caspase-2 is involved in initiating irofulven-induced apoptosis.

\section{Figure 2A}

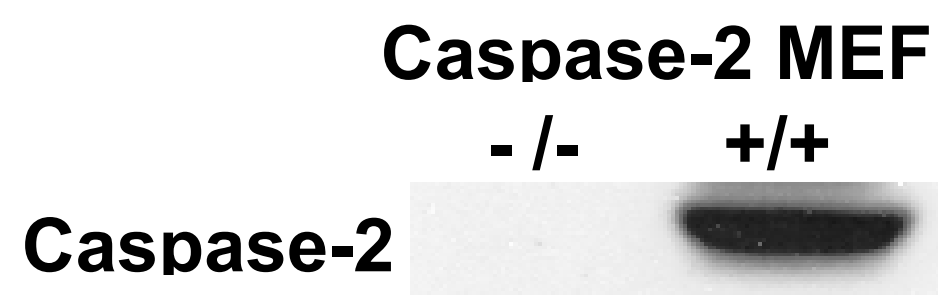

Actin

Figure 2B

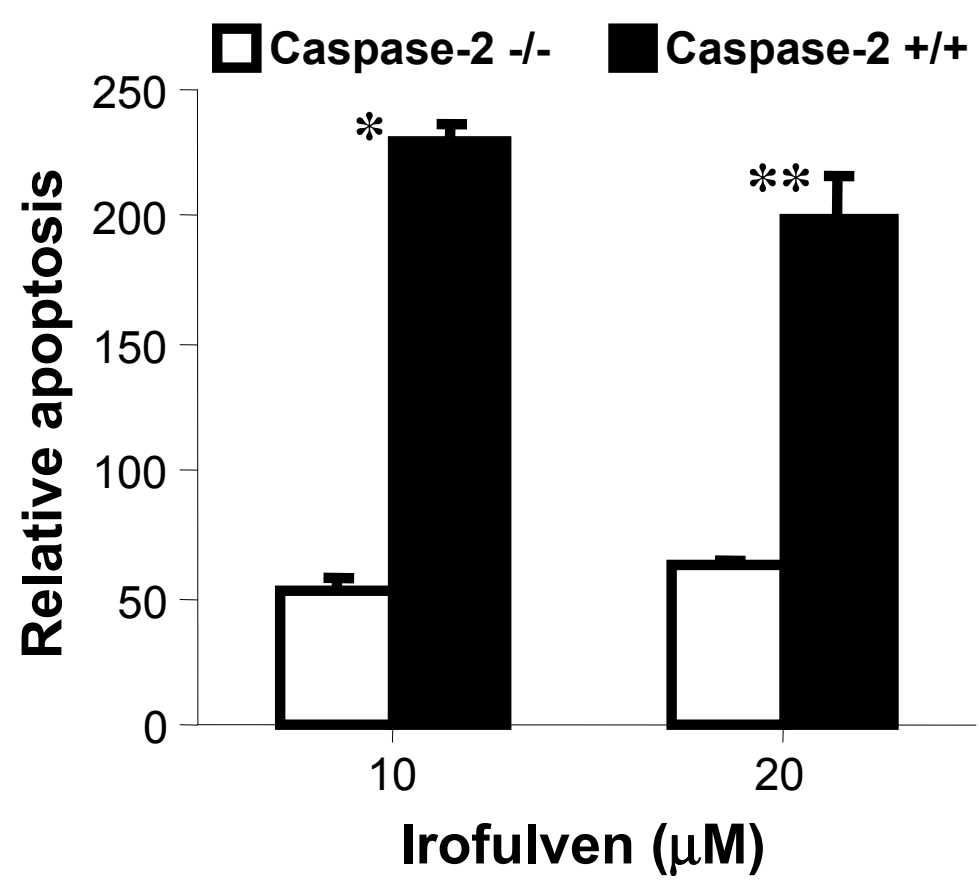


Figure 2C

Caspase-2 -/- Caspase-2 +/+
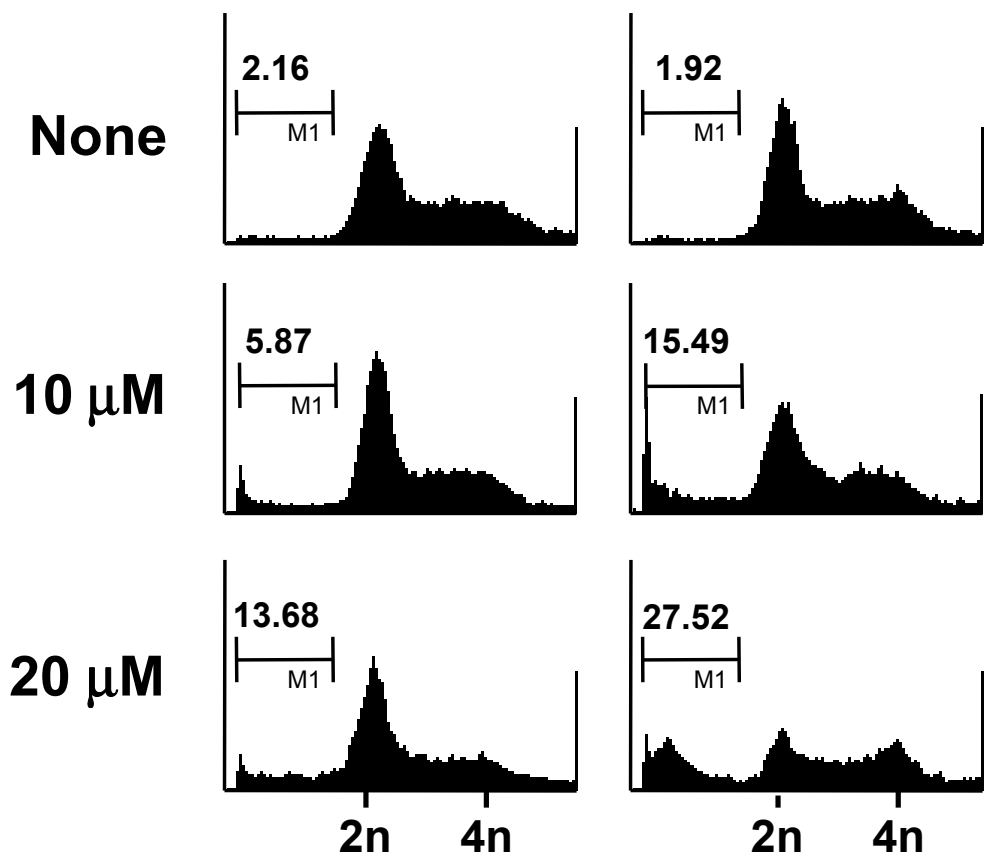

Figure 2D

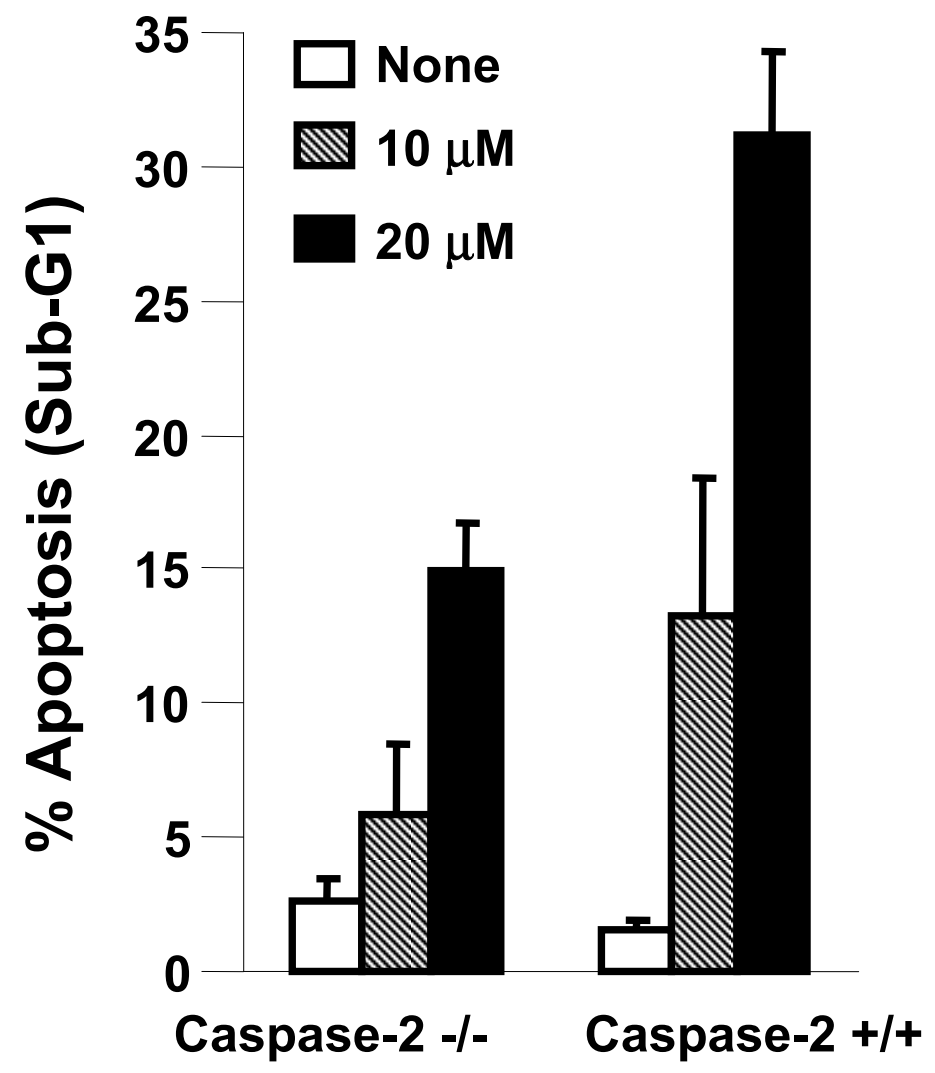


Figure 3. Irofulven activates caspase-9 of the mitochondrial apoptosis pathway.

Figure 3A

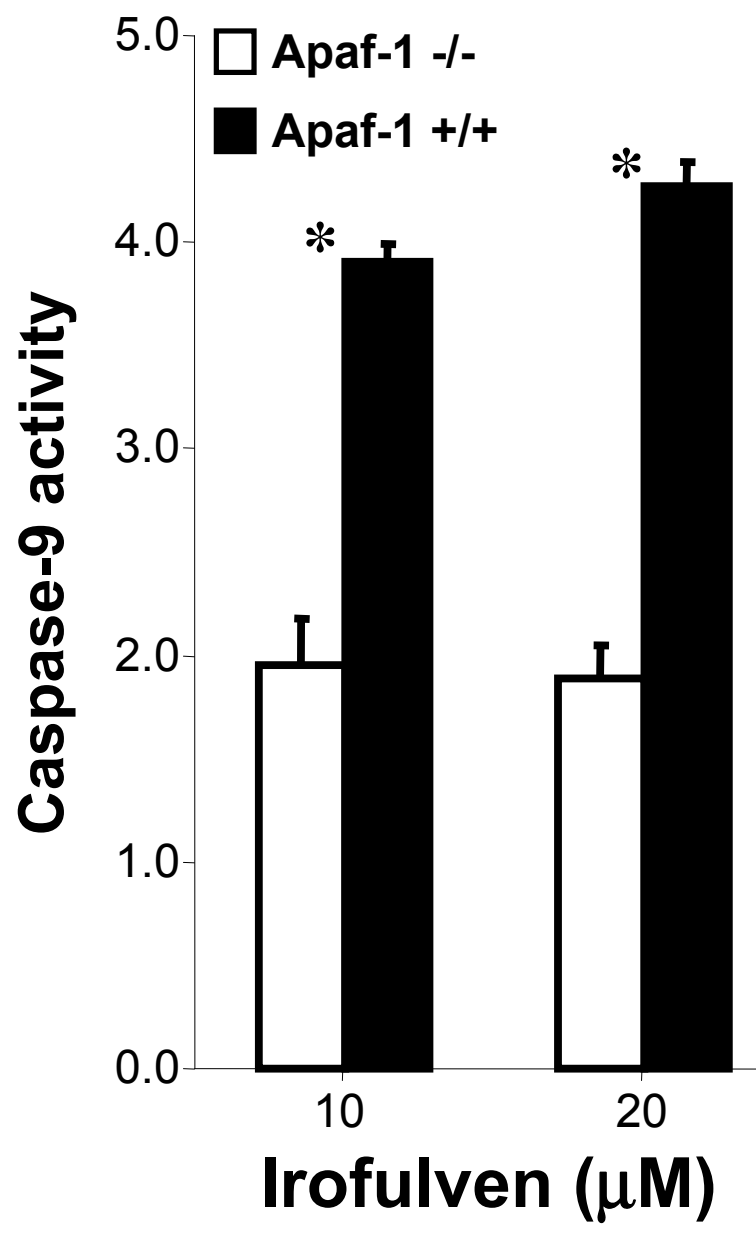


Figure 3B

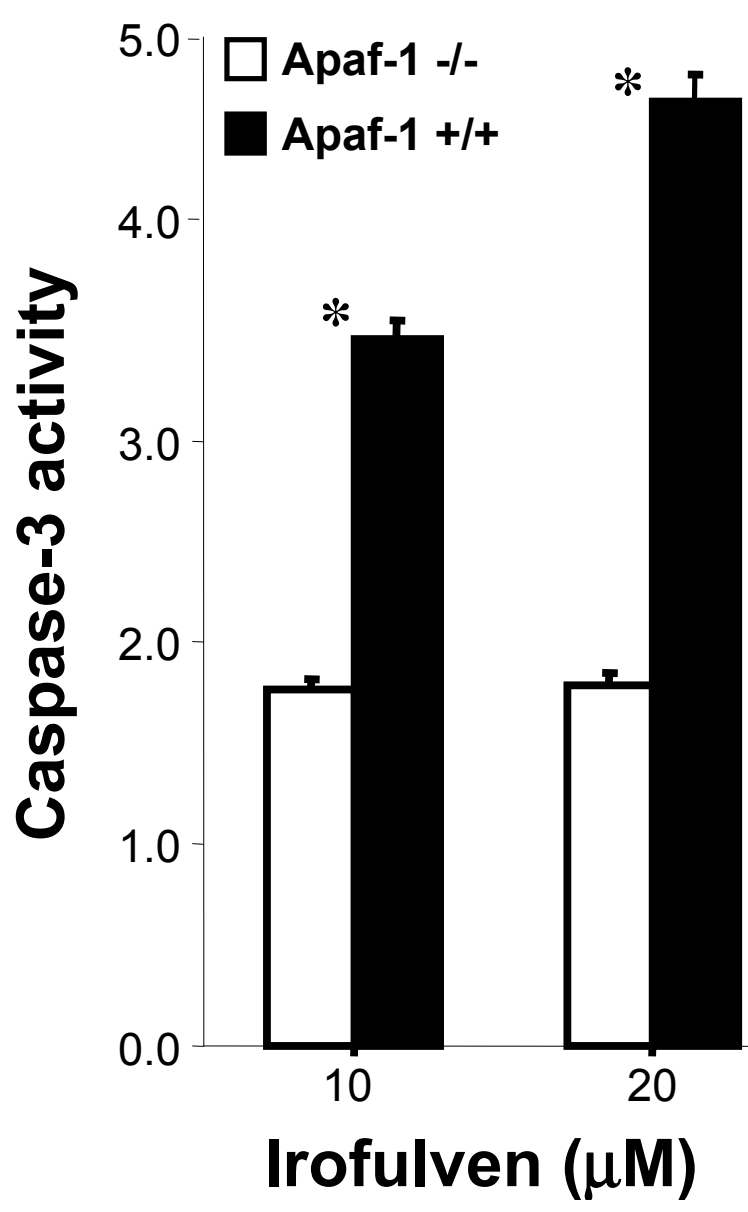


Figure 4A

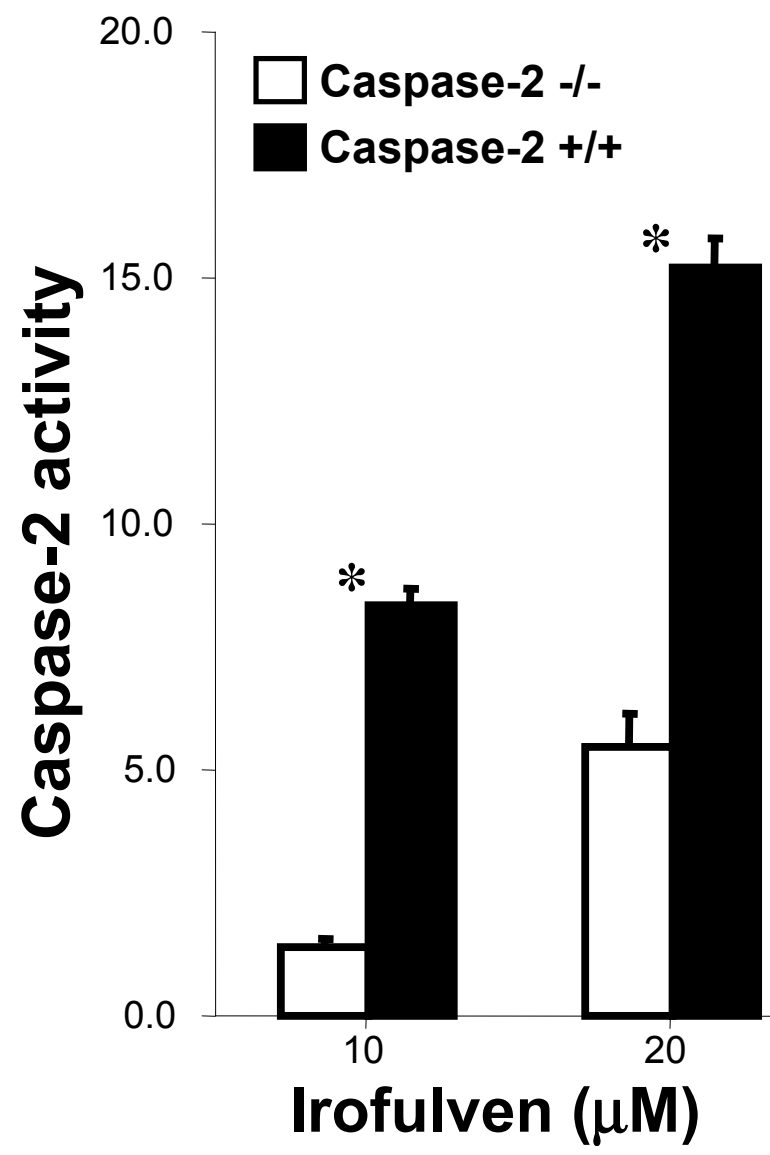


Figure 4B

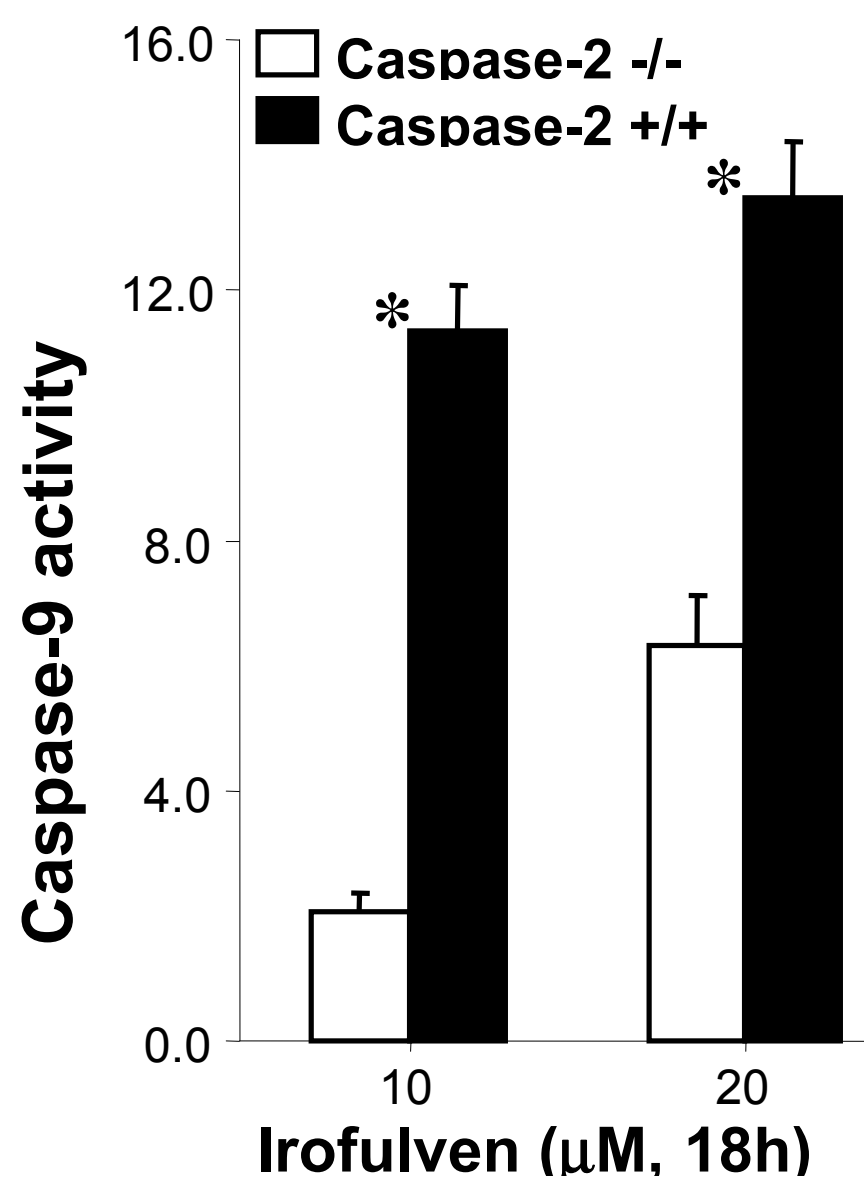


Figure 4C

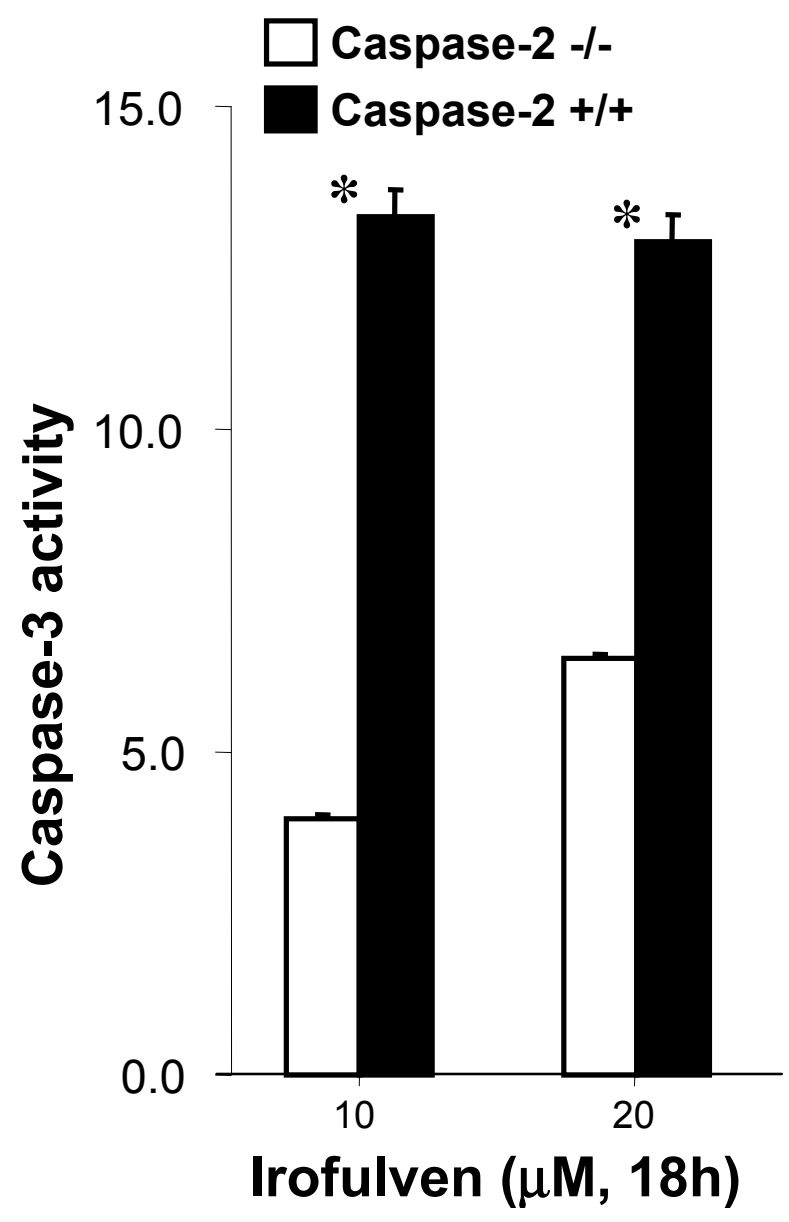


Figure 5. Caspase-2 initiated mitochondrial apoptosis pathway enhances chemosensitivity to irofulven.

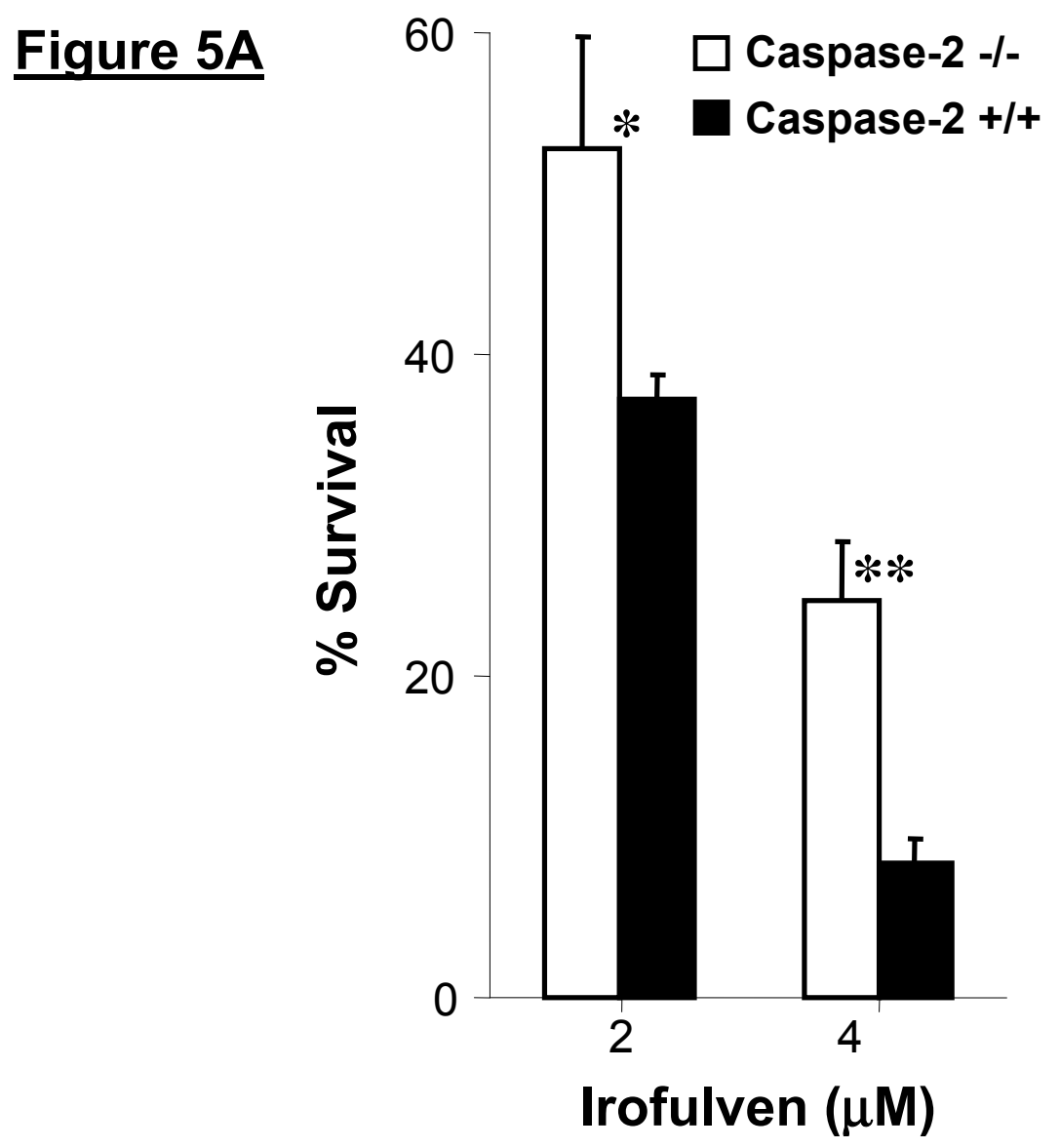


Figure 5B

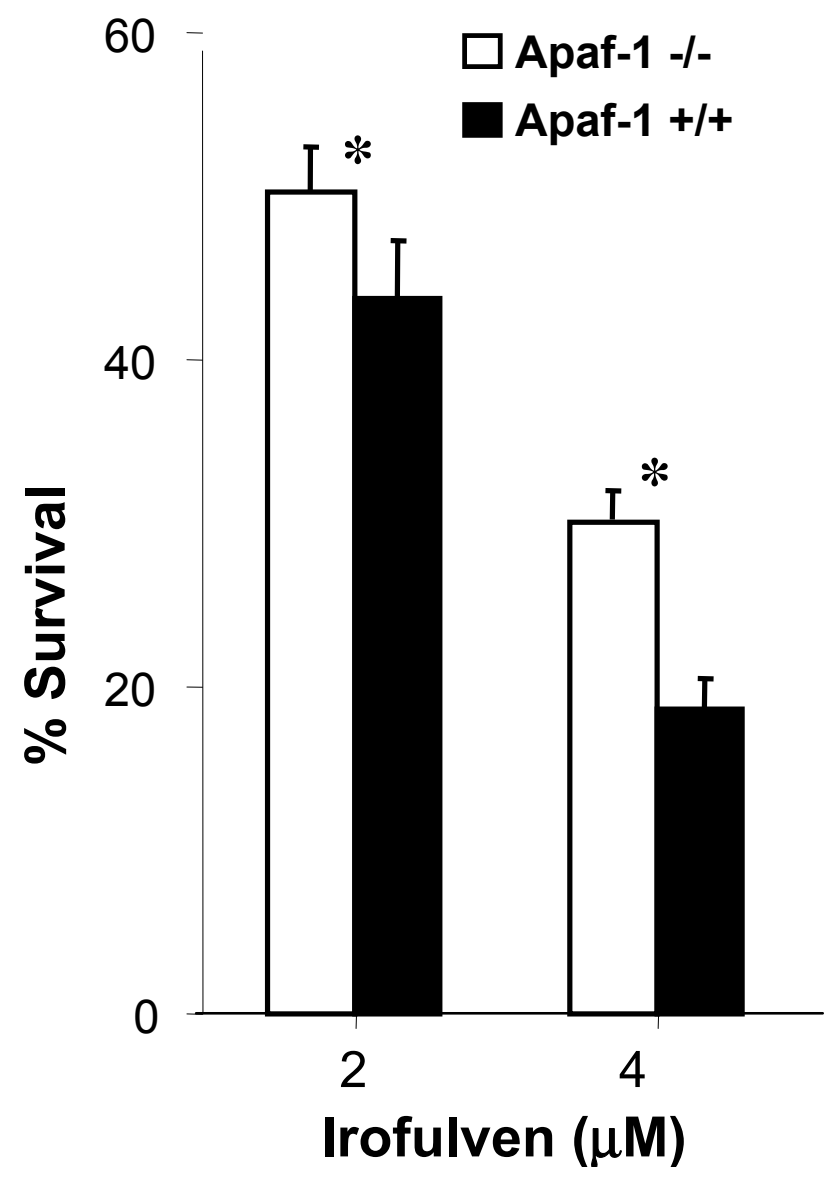




\section{Chapter IV}

\section{Role of Caspase 8 in Irofulven Induced Apoptosis}




\section{Abstract}

Induction of apoptosis by chemotherapeutic agents remains a highly desirable and effective trait. The goal of many current therapies is to activate the apoptotic response in tumor cells that have lost this normal cellular function. Irofulven (6hydroxymethylacylfulvene, MGI 114, NSC\#: 683863) is one of a new class of anticancer agents that are analogs of the mushroom derived illudin $\mathrm{S}$ toxins. Preclinical and clinical trials have shown that irofulven is effective against several solid tumor types. It has been shown to activate several proteins in the DNA damage response as well as MAP kinase signaling and apoptosis leading to cell cycle arrest and modulating chemosensitivity. In this study, we investigated the role of caspase 8 in irofulven induced apoptosis. We also examined the role that ERK may play in this response. Using both mouse embryo fibroblasts and neuroblastoma cells proficient and deficient for caspase 8, we demonstrated that cells lacking caspase 8 function were more sensitive to treatment with irofulven. We demonstrated an undefined survival effect whereby caspase 8 protects cells from irofulven induced apoptosis. We also determined that activation of ERK was serving as a pro-apoptotic mechanism with some dependence on caspase 8 . Furthermore, we established that caspase 8 rendered cells more chemoresistant to irofulven. 


\section{Introduction}

Caspase dependent apoptosis is a form of programmed cell death controlled by a pathway of cysteine proteases known as caspases. It is characterized by shrinkage in overall cellular size, condensation of chromatin and DNA fragmentation (1-3). The structure of caspase family members is similar in that most contain pro-domains as well as a small and large subunit to make the full length single chain protein (4). They are synthesized as inactive zymogens and enzymatic activation is achieved through cleavage at specific sites in the N-terminal, after an aspartic acid residue $(4,5)$. Both an intrinsic and extrinsic pathway of activation exists, merging with the activation of the effector caspases 3, 6 and 7 .

The death receptor mediated pathway of apoptosis is a classical pathway by which cells are able to respond to extracellular signals and subsequently activate programmed cell death. A number of ligands, including Fas, TNF and TRAIL, have been identified that are able to activate transmembrane receptors which begins the signaling cascade that leads to apoptosis (6). After receptor engagement, the cytoplasmic domain of the receptor is able to bind a number of FADD (Fas-associated death domain) adaptor molecules through homotypic interaction between the death domain (DD) of both Fas and FADD (7, 8). The N-terminal portion of FADD contains a death effector domain (DED) that acts to recruit caspase 8 to the complex and forms an interaction with the DED of procaspase 8 $(7,9)$. This complex assembled with several molecules of Fas, FADD and procaspase 8 forms a functional apoptotic complex called the death-inducing signaling complex (DISC) (10). Oligomerization of procaspase 8 with adaptor molecules at receptor sites 
leads to autoproteolytic cleavage of caspase 8 and subsequent activation of effector caspases (11). Evidence also exists demonstrating the ability of caspase 8 to cleave Bid resulting in release of cytochrome $\mathrm{c}$ and activation of the mitochondrial mediated pathway (12).

Aside from a role in apoptosis, caspases have been shown to be involved in a number of other cellular processes including embryonic development, macrophage differentiation, $\mathrm{T}$ cell proliferation and cell motility (13-20). The differentiation of monocytes into mature macrophages during the process of infection occurs in the presence of several active caspases while not exhibiting the morphological characteristics that are a hallmark of apoptosis (17). Other evidence also revealed a mechanism by which caspase 3 is able to drive platelet formation in the absence of detectable DNA fragmentation (21). Recent evidence has defined a role for caspase 8 in cell motility in caspase 8 null mouse embryo fibroblasts under nonapoptotic conditions (14). It should also be noted that caspase 8 has recently been linked to an increased risk of breast cancer raising more questions as to what other functions caspase 8 may play in the cell (22).

Activation of the apoptotic pathway by chemotherapeutic agents is a highly desirable and effective trait. Several current therapies rely on the induction of apoptosis to kill cancerous cells. Many DNA damaging agents have been shown to be potent inducers of apoptosis (23). While studies have illustrated the induction of apoptosis by DNA damaging agents, several critical steps in the process remain unclear. The mitochondrial controlled pathway is a key component in the induction of apoptosis in response to ionizing radiation and several chemotherapeutics $(1,24-26)$. What remains 
unclear is whether the mitochondrial pathway acts alone or in conjunction with the death receptor mediated pathway.

Irofulven (6-hydroxymethylacylfulvene, MGI 114, NSC\#: 683863) is one of a new class of anticancer agents that are analogs of the mushroom derived illudin $\mathrm{S}$ toxins. Preclinical and clinical trials have shown that irofulven is effective against several solid tumor types (27-33). The exact mechanism of action of irofulven remains mostly unknown. While previous studies have suggested that damage induced by irofulven is repaired exclusively via the transcription coupled nucleotide excision repair (TC-NER) pathway, these studies have ignored the NHEJ and HR pathways (34). Recent work in our lab has shown that irofulven activates ATM and its downstream targets: BRCA1, NBS1, FANCD2, SMC1, CHK2 and p53 (35-37). Although most data shows that irofulven is able to cause DNA damage, the precise mechanism of killing remains largely unknown. Previous studies have shown induction of apoptosis mediated by ERK and JNK kinases (38). It has also been shown that irofulven treatment induces the cleavage of caspases 8 and 9 (39). This data has been corroborated by other evidence that caspase induced apoptosis plays a role in cell death after irofulven treatment (40).

Previous work in our lab, as reported in Chapter III of this dissertation, has demonstrated the ability of irofulven to activate apoptosis via caspase 2 and dependent on the mitochondrial pathway. In this study, we sought to determine the role of the death receptor mediated apoptotic pathway after irofulven treatment. We used isogenic mouse embryonic fibroblasts proficient and deficient for caspase 8 to demonstrate that cells lacking caspase 8 function actually activated apoptosis more strongly than caspase 8 proficient cells. We verified this phenomenon using NB7 neuroblastoma cells, which lack 
expression of the caspase 8 protein, paired with NB7 cells with reconstituted caspase 8 function. 


\section{Materials and Methods}

\section{Cell culture and Reagents}

Mouse embryonic fibroblasts (MEFs) knocking-out caspase-8 were cultured in DMEM (Mediatech, Herndon, VA) supplemented with 10\% fetal bovine serum and penicillin/streptomycin in a $37^{\circ} \mathrm{C}$ incubator with $5 \% \mathrm{CO}_{2}$ atmosphere. Previously described NB7 cells were cultured under the same conditions in RPMI (Mediatech, Herndon, VA) media supplemented with $10 \%$ fetal bovine serum and penicillin/streptomycin (41).

\section{Apoptosis ELISA}

Apoptosis ELISA was performed using the Cell Death Detection ELISA ${ }^{\text {PLUS }}$ kit (Roche, Indianapolis, IN) according to manufacturer's instructions to detect cytoplasmic histone-associated DNA fragments (mono- and oligonucleosomes). Cells were plated in 6-well plates and allowed to attach overnight. Treatment with irofulven was done with various doses and exposure times as noted. After drug exposure, both the floating and adherent cells were collected and washed with ice-cold PBS. Cell pellets were then lysed in provided lysis buffer for $30 \mathrm{~min}$ at room temperature followed by $10 \mathrm{~min}$ of centrifugation at $1500 \mathrm{~g}$. The supernatant $(20 \mu \mathrm{l})$ was added to each well along with $80 \mu \mathrm{l}$ of immunoreagent consisting of anti-DNA and anti-POD antibodies. Microplate was covered and allowed to shake at $300 \mathrm{rpm}$ for two hours at room temperature. Following incubation, the wells were washed three times with incubation buffer and $100 \mu 1$ of ABTS 
solution was added. Microplate was allowed to shake at $250 \mathrm{rpm}$ for 5-10 minutes and then read at $405 \mathrm{~nm}$ on a spectrophotometer (Molecular Devices, Sunnyvale, CA). Results are reported as fold change from untreated cells minus background and represent three independent experiments.

\section{Western blotting}

Western blot was performed as described previously (42). Whole cell lysates (100 $\mu g)$ were separated by SDS-PAGE. Primary antibodies used were: rat anti-mouse caspase 2 (1:1000), rat anti-mouse caspase 8 (1:1000; Alexis Biochemicals, San Diego, CA) mouse anti-human caspase 8 (1:500; BD Biosciences, San Jose, CA), rabbit anti-Apaf-1 (1:100; Santa Cruz Biotechnology, Santa Cruz, CA), mouse anti-pERK (E-4), goat antiERK2 (C-14) (1:1000, Santa Cruz Biotechnology, Santa Cruz, CA) and mouse anti-actin (1:5000; Sigma, St. Louis, MO). Secondary antibodies used were: sheep anti-mouse IgGHRP, sheep anti-rabbit IgG-HRP (1:4,000; GE Healthcare, Piscataway, NJ) and goat antirat IgG-HRP (1:4000; Santa Cruz Biotechnology, Santa Cruz, CA). All dilutions were done as indicated in $1 \mathrm{X}$ PBS, $0.1 \%$ Tween 20 and 5\% milk.

\section{Clonogenic survival assay}

To determine chemosensitivity, clonogenic survival assay was performed as previously described (42). Cells were plated in $60 \mathrm{~mm}$ dishes overnight in complete medium and were treated with different concentrations of irofulven for $24 \mathrm{~h}$. Medium was then replaced with fresh drug-free medium and incubated for 7-10 days. Colonies were stained with PBS containing $0.04 \%$ crystal violet and $0.5 \%$ paraformaldehyde for 
about 10 min. Staining liquid was aspirated and colonies counted. Results are reported as treated divided by untreated control and represent 3 individual experiments.

\section{Annexin V Staining}

Analysis of cells undergoing apoptosis was measured by Annexin V staining and flow cytometry using a commercial kit from Sigma (St. Louis, MO) and according to the manufacturer's protocol. Cells were treated with irofulven for 18 hours and harvested by mechanical detachment. Cells were then washed with PBS and re-suspended in the supplied $1 \mathrm{X}$ binding buffer followed by the addition of $5 \mu \mathrm{L}$ of annexin V-FITC and 10 $\mu \mathrm{L}$ of propidium iodide. Samples were incubated for $10 \mathrm{~min}$ at room temperature in the dark followed by measurement of annexin V and PI content (20,000 events each) using a FACSCalibur cytometer with CellquestPro software (BD Biosciences, San Jose, CA). Cells that are in the early stages of or already undergoing apoptosis will stain positive for only the annexin V-FITC. Necrotic cells will stain positive for both annexin V-FITC and PI due the compromised integrity of the membrane. Live cells will show no staining by either annexin V-FITC or PI. 


\section{Results}

\section{Irofulven induced apoptosis is independent of caspase 8}

Although it's role is somewhat controversial, activation of the death receptor mediated pathway of apoptosis has been shown to modulate chemosensitivity in several tumor types (43-49). In this study, we sought to define the role of caspase 8 in irofulven induced apoptosis. Our first model for activation of the death receptor pathway was caspase 8 -/- and +/+ MEFs. We first verified the status of caspase 8 in these cells with western blot analysis (Figure 1A). We then used a DNA fragmentation ELISA to determine the induction of apoptosis after irofulven treatment. Using TNF $\alpha$ as a positive

control, we observed that caspase 8 proficient cells actually underwent less activation of apoptosis than their caspase 8 deficient counterpart at both 5 and $10 \mu \mathrm{M}$ doses (Figure 1B). To determine whether this phenomenon translated into increased survival, we performed clonogenic survival assay by treatment of the caspase $8-/-$ and $+/+$ cells for 24 hours followed by incubation for several days. Consistent with earlier results, we observed that caspase $8+/+$ cells realized increased survival after irofulven treatment.

Given the fact that our observations were unique and unexpected, we had to consider the possibility that this was a cell type specific response that was exclusive to the set of MEFs that we used in these experiments. To verify the results, we chose the NB7 neuroblastoma cell line that lacks caspase 8 function. When paired with a wild type reconstituted NB7 cell line, this made another good model system to study the role of caspase 8 in irofulven induced apoptosis. We first verified the status of the caspase 8 protein in these cells (Figure 2A). When then used the DNA fragmentation ELISA to 
determine induction of apoptosis. Using TNF $\alpha$ as our positive control, we once again observed that cells lacking caspase 8 activity induced apoptosis to a greater degree than caspase 8 proficient cells. At both 5 and $10 \mu \mathrm{M}$ doses, the NB7- cells showed increased DNA fragmentation after treatment with irofulven (Figure 2B). To verify these results, we also used annexin V and flow cytometry analysis to measure apoptosis in the NB7 set of cells. Consistent with our previous experiments, TNF $\alpha$ preferentially activated apoptosis in NB7+C8 cells while irofulven treatment resulted in increased levels of annexin V positive cells in NB7- cells. A representative histogram of the results is shown in figure $2 \mathrm{C}$ and a graphical illustration depicting the mean of triplicate experiments is shown in figure 2D. This set of experiments provided strong evidence for a protective role of caspase 8 in response to irofulven treatment.

\section{Role of ERK in irofulven induced apoptosis}

We next set out to determine what could be modulating this unusual phenomenon of caspase 8 dependent protection from irofulven induced apoptosis. We decided to look at ERK as a possible regulator based on the fact that caspase 8 has been shown to promote cell motility along with the observation that caspase 8 is able to regulate ERK phosphorylation under nonapoptotic conditions (14) (Steve Frisch, personal communication). These data provide a plausible link from caspase 8 to MAP kinase signaling leading to increased tumor aggressiveness. We used a phospho-ERK specific antibody to probe irofulven treated NB7- and NB7+C8 cells. Our results did indicate a slight increase of pERK at the $4 \mathrm{~h}$ timepoint with negligible differences at 18 and 24 hours (Figure 3A). 
Based on these results, we did similar analysis of caspase $8-/-$ and $+/+$ cells at early timepoints. We observed that pERK levels in the caspase $8+/+$ cells seemed to be increased over the caspase 8 -/- cells at 4 hours when treated with the $20 \mu \mathrm{M}$ dose (Figure 3B). Using the $30 \mu \mathrm{M}$ dose, we treated cells with irofulven and harvested at one hour intervals up to 4 hours to determine a timeline for pERK activity. Interestingly, we observed early induction of ERK phosphorylation in both cells at 1 hour followed by a drop in pERK levels at 2 hours (Figure 3C). After the initial phosphorylation then drop, the caspase $8+/+$ cells showed increased pERK levels at 3 and 4 hours, whereas the caspase 8 -/- cells only showed a slight increase again at 4 hours. This biphasic response was reproducible by western blot analysis with variable times for initial phosphorylation followed by a drop in pERK levels only to increase again within 3-4 hours. These results demonstrate a role for ERK activity in response to irofulven treatment that is not well defined.

To determine how modulation of ERK activity was affecting induction of apoptosis, we used the MEK inhibitor PD98059 in conjunction with apoptosis ELISA to measure how DNA fragmentation changed when irofulven treated cells were pretreated with the inhibitor. In our caspase 8 -/- and +/+ MEFs, we pretreated for 30 minutes with PD98059 before addition of irofulven. Measurement of apoptosis revealed that inhibition of ERK lead to a decrease in apoptosis with some dependence on caspase 8 (Figure 3D). Caspase $8+/+$ cells treated with PD nearly shut down apoptosis completely where as caspase 8 -/- cells showed only a slight decrease. The PI3 kinase inhibitor LY294002 was also used in this experiment to rule out activation of the PI3K pathway as a survival signal. 


\section{Discussion}

In this study, we demonstrated that not only does caspase 8 not sensitize cells to irofulven induced apoptosis, but it leads to protection from it. We established that caspase 8 -/- MEFs induced more apoptosis and were more chemosensitive than caspase $8+/+$ cells. Given this unique response to irofulven, we also corroborated this phenomenon in NB7- and NB7+C8 cells.

Although the mitochondrial pathway is thought to play a major role in response to many chemotherapeutics, a number of anticancer agents have been shown to induce apoptosis in tumors dependent on the activity of caspase 8 and the death receptor mediated pathway $(44,45,50,51)$. This led us to explore a role for caspase 8 in irofulven induced apoptosis. A protective role for caspase 8 was not expected but is not without merit. Recent mounting evidence has linked several members of the caspase family to nonapoptotic cellular roles including embryonic development, macrophage differentitation, $\mathrm{T}$ cell proliferation and cell motility (13-20). More specifically, in caspase 8 null mouse embryo fibroblasts, caspase 8 was shown to contribute to increased cell motility under nonapoptotic conditions (14). Our observation that caspase 8 leads to a protective effect after treatment with irofulven, adds to the notion that caspase 8 may have an as of yet undefined function relating to cell survival. We sought to determine the role of ERK activity in response to irofulven treatment based on data implicating caspase 8 dependent phosphorylation of ERK under nonapoptotic conditions (Steve Frisch, personal communication). As our results indicate, ERK seems to be acting as proapoptotic signal as when we pretreat using the MEK inhibitor, PD98059, we severely 
inhibit the induction of apoptosis. This led us to rule out the possibility that ERK was acting as a survival signal and opened up a multitude of other possible signals to explore.

In support of the notion that an undefined survival signal is being activated, during our experiments carried out with irofulven treatment in conjunction with PD98059, we actually saw an increase in cell proliferation in the irofulven and PD treated group as compared to the untreated control (unpublished observation). Whereas the untreated group were about $60 \%$ confluent in this experiment, the irofulven + PD group reached $100 \%$ confluency indicating the activation of an undefined survival signal. This also led us to the conclusion that ERK was acting as a strong proapoptotic signal in response to irofulven. This observation is substantiated by a number of studies that outline a proapoptotic function for ERK in response to drug treatment (52-54). It must also be noted that ERK has been reported to abrogate the Fas-mediated death signal by inhibition of Bid cleavage by caspase 8 while not affecting DISC assembly $(55,56)$. The opposite effect seen in various cells types could very well be a cell type specific mechanism much like caspase 8 mediated killing can be differentiated into type I and II cells. Much remains to be determined about interactions between caspase 8 and ERK including what role they play in modulating apoptosis.

In summary, our current study has revealed a role for caspase 8 that protects MEFs and NB7 cells from irofulven induced apoptosis. We have also demonstrated that ERK activation leads to apoptosis as evidenced by increased survival when we block with the PD98059 MEK inhibitor. The interesting phenomenon that remains to be elucidated is what sort of signal could be activated in a caspase 8 dependent manner leading to increased survival of caspase 8 proficient cells in response to irofulven. 


\section{References}

1. Thornberry, N. A. and Lazebnik, Y. Caspases: Enemies Within. Science, 281: 1312-1316, 1998.

2. Green, D. R. Apoptotic pathways: paper wraps stone blunts scissors. Cell, 102: 14, 2000

3. Roth, W. and Reed, J. C. Apoptosis and cancer: when BAX is TRAILing away. Nat Med, 8: 216-218, 2002.

4. Nicholson, D. W. and Thornberry, N. A. Caspases: killer proteases. Trends Biochem Sci, 8: 299-306, 1997.

5. Cohen, G. M. Caspases: the executioners of apoptosis. Biochem. J., 326: 1-16, 1997.

6. Ashkenazi, A. and Dixit, V. M. Death receptors: signaling and modulation. Science, 281: 1305-1308, 1998.

7. Boldin, M. P., Goncharov, T. M., Goltsev, Y. V., and Wallach, D. Involvement of MACH, a novel MORT1/FADD-interacting protease, in Fas/APO-1- and TNF receptor-induced cell death. Cell, 85: 803-815, 1996.

8. Chinnaiyan, A. M., O'Rourke, K., Tewari, M., and Dixit, V. FADD, a novel death domain-containing protein, interacts with the death domain of Fas and initiates apoptosis. Cell, 81: 505-512, 1995. 
9. Muzio, M., Chinnaiyan, A. M., Kischkel, F. C., O'Rourke, K., Shevchenko, A., Ni, J., Scaffidi, C., Bretz, J. D., Zhang, M., Gentz, R., Mann, M., Krammer, P. H., Peter, M. E., and Dixit, V. FLICE, a novel FADD-homologous ICE/CED-3-like protease, is recruited to the CD95 (Fas/APO-1) death--inducing signaling complex. Cell, 85: 817-827, 1996.

10. Kischkel, F. C., Hellbardt, S., Behrmann, I., Germer, M., Pawlita, M., Krammer, P. H., and Peter, M. E. Cytotoxicity-dependent APO-1 (Fas/CD95)-associated proteins form a death-inducing signaling complex (DISC) with the receptor. EMBO Journal, 14: 5579-5588, 1995.

11. Yang, X., Chang, H. Y., and Baltimore, D. Autoproteolytic Activation of ProCaspases by Oligomerization. Molecular Cell, 1: 319-325, 1998.

12. Li, H., Zhu, H., Xu, C. J., and Yuan, J. Cleavage of BID by caspase 8 mediates the mitochondrial damage in the Fas pathway of apoptosis. Cell, 94: 491-501, 1998.

13. Kang, T.-B., Ben-Moshe, T., Varfolomeev, E. E., Pewzner-Jung, Y., Yogev, N., Jurewicz, A., Waisman, A., Brenner, O., Haffner, R., Gustafsson, E., Ramakrishnan, P., Lapidot, T., and Wallach, D. Caspase-8 Serves Both Apoptotic and Nonapoptotic Roles. J Immunol, 173: 2976-2984, 2004.

14. Helfer, B., Boswell, B. C., Finlay, D., Cipres, A., Vuori, K., Bong Kang, T., Wallach, D., Dorfleutner, A., Lahti, J. M., Flynn, D. C., and Frisch, S. M. 
Caspase-8 Promotes Cell Motility and Calpain Activity under Nonapoptotic Conditions. Cancer Res, 66: 4273-4278, 2006.

15. Kennedy, N. J., Kataoka, T., Tschopp, J., and Budd, R. C. Caspase Activation Is Required for T Cell Proliferation. J. Exp. Med., 190: 1891-1896, 1999.

16. Boissonnas, A., Bonduelle, O., Lucas, B., Debré, P., Autran, B., and Combadière, B. Differential requirement of caspases during naive T cell proliferation. European Journal of Immunology, 32: 3007-3015, 2002.

17. Sordet, O., Rebe, C., Plenchette, S., Zermati, Y., Hermine, O., Vainchenker, W., Garrido, C., Solary, E., and Dubrez-Daloz, L. Specific involvement of caspases in the differentiation of monocytes into macrophages. Blood, 100: 4446-4453, 2002.

18. De Maria, R., Zeuner, A., Eramo, A., Domenichelli, C., Bonci, D., Grignani, F., Srinivasula, S. M., Alnemri, E. S., Testa, U., and Peschle, C. Negative regulation of erythropoiesis by caspase-mediated cleavage of GATA-1. 401: 489-493, 1999.

19. Zermati, Y., Garrido, C., Amsellem, S., Fishelson, S., Bouscary, D., Valensi, F., Varet, B., Solary, E., and Hermine, O. Caspase Activation Is Required for Terminal Erythroid Differentiation. J. Exp. Med., 193: 247-254, 2001.

20. Ishizaki, Y., Jacobson, M. D., and Raff, M. C. A Role for Caspases in Lens Fiber Differentiation. J. Cell Biol., 140: 153-158, 1998.

21. de Botton, S., Sabri, S., Daugas, E., Zermati, Y., Guidotti, J. E., Hermine, O., Kroemer, G., Vainchenker, W., and Debili, N. Platelet formation is the 
consequence of caspase activation within megakaryocytes. Blood, 100: 13101317, 2002.

22. Cox, A., Dunning, A. M., Garcia-Closas, M., Balasubramanian, S., Reed, M. W. R., Pooley, K. A., Scollen, S., Baynes, C., Ponder, B. A. J., Chanock, S., Lissowska, J., Brinton, L., Peplonska, B., Southey, M. C., Hopper, J. L., McCredie, M. R. E., Giles, G. G., Fletcher, O., Johnson, N., dos Santos Silva, I., Gibson, L., Bojesen, S. E., Nordestgaard, B. G., Axelsson, C. K., Torres, D., Hamann, U., Justenhoven, C., Brauch, H., Chang-Claude, J., Kropp, S., Risch, A., Wang-Gohrke, S., Schurmann, P., Bogdanova, N., Dork, T., Fagerholm, R., Aaltonen, K., Blomqvist, C., Nevanlinna, H., Seal, S., Renwick, A., Stratton, M. R., Rahman, N., Sangrajrang, S., Hughes, D., Odefrey, F., Brennan, P., Spurdle, A. B., Chenevix-Trench, G., Beesley, J., Mannermaa, A., Hartikainen, J., Kataja, V., Kosma, V.-M., Couch, F. J., Olson, J. E., Goode, E. L., Broeks, A., Schmidt, M. K., Hogervorst, F. B. L., Veer, L. J. V. t., Kang, D., Yoo, K.-Y., Noh, D.-Y., Ahn, S.-H., Wedren, S., Hall, P., Low, Y.-L., Liu, J., Milne, R. L., Ribas, G., Gonzalez-Neira, A., Benitez, J., Sigurdson, A. J., Stredrick, D. L., Alexander, B. H., Struewing, J. P., Pharoah, P. D. P., and Easton, D. F. A common coding variant in CASP8 is associated with breast cancer risk. 39: 688, 2007.

23. Norbury, C. J. and Zhivotovsky, B. DNA damage-induced apoptosis. Oncogene, 23: 2797-2808, 2004. 
24. Yoshida, H., Kong, Y.-Y., Yoshida, R., Elia, A. J., Hakem, A., Hakem, R., Penninger, J. M., and Mak, T. W. Apaf1 Is Required for Mitochondrial Pathways of Apoptosis and Brain Development. Cell, 94: 739-750, 1998.

25. Hakem, R., Hakem, A., Duncan, G. S., Henderson, J. T., Woo, M., Soengas, M. S., Elia, A., de la Pompa, J. L., Kagi, D., Khoo, W., Potter, J., Yoshida, R., Kaufman, S. A., Lowe, S. W., Penninger, J. M., and Mak, T. W. Differential Requirement for Caspase 9 in Apoptotic Pathways In Vivo. Cell, 94: 339-352, 1998.

26. Soengas, M. S., Alarcon, R. M., Yoshida, H., Giaccia, A. J., Hakem, R., Mak, T. W., and Lowe, S. W. Apaf-1 and Caspase-9 in p53-Dependent Apoptosis and Tumor Inhibition. Science, 284: 156-159, 1999.

27. Hidalgo, M., Izbicka, E., Eckhardt, S. G., MacDonald, J. R., Cerna, C., Gomez, L., Rowinsky, E. K., Weitman, S. D., and Von Hoff, D. D. Antitumor activity of MGI 114 (6-hydroxymethylacylfulvene, HMAF), a semisynthetic derivative of illudin S, against adult and pediatric human tumor colony-forming units. Anticancer Drugs, 10: 837-844, 1999.

28. MacDonald, J. R., Muscoplat, C. C., Dexter, D. L., Mangold, G. L., Chen, S. F., Kelner, M. J., McMorris, T. C., and Von Hoff, D. D. Preclinical antitumor activity of 6-hydroxymethylacylfulvene, a semisynthetic derivative of the mushroom toxin illudin S. Cancer Res, 57: 279-283, 1997. 
29. Sato, Y., Kashimoto, S., MacDonald, J. R., and Nakano, K. In vivo antitumour efficacy of MGI-114 (6-hydroxymethylacylfulvene, HMAF) in various human tumour xenograft models including several lung and gastric tumours. Eur J Cancer, 37: 1419-1428, 2001.

30. Senzer, N., Arsenau, J., Richards, D., Berman, B., MacDonald, J. R., and Smith, S. Irofulven demonstrates clinical activity against metastatic hormone-refractory prostate cancer in a phase 2 single-agent trial. Am J Clin Oncol, 28: 36-42, 2005.

31. Thomas, J. P., Arzoomanian, R., Alberti, D., Feierabend, C., Binger, K., Tutsch, K. D., Steele, T., Marnocha, R., Smith, C., Smith, S., MacDonald, J., Wilding, G., and Bailey, H. Phase I clinical and pharmacokinetic trial of irofulven. Cancer Chemother Pharmacol, 48: 467-472, 2001.

32. Giles, F., Cortes, J., Garcia-Manero, G., Kornblau, S., Estey, E., Kwari, M., Murgo, A., and Kantarjian, H. Phase I study of irofulven (MGI 114), an acylfulvene illudin analog, in patients with acute leukemia. Invest New Drugs, 19: 13-20, 2001.

33. Murgo, A., Cannon, D. J., Blatner, G., and Cheson, B. D. Clinical trials referral resource. Clinical trials of MGI-114. Oncology (Huntingt), 13: 233, 237-238, 1999.

34. Jaspers, N. G., Raams, A., Kelner, M. J., Ng, J. M., Yamashita, Y. M., Takeda, S., McMorris, T. C., and Hoeijmakers, J. H. Anti-tumour compounds illudin S and Irofulven induce DNA lesions ignored by global repair and exclusively processed 
by transcription- and replication-coupled repair pathways. DNA Repair (Amst), 1: 1027-1038, 2002.

35. Wang, J., Wiltshire, T., Wang, Y., Mikell, C., Burks, J., Cunningham, C., Van Laar, E. S., Waters, S. J., Reed, E., and Wang, W. ATM-dependent CHK2 Activation Induced by Anticancer Agent, Irofulven. J Biol Chem, 279: 3958439592, 2004.

36. Wang, Y., Wiltshire, T., Senft, J., Reed, E., and Wang, W. Irofulven induces replication-dependent CHK2 activation related to p53 status. Biochem Pharmacol, 73: 469-480, 2007.

37. Wang, Y., Wiltshire, T., Senft, J., Wenger, S. L., Reed, E., and Wang, W. Fanconi anemia D2 protein confers chemoresistance in response to the anticancer agent, irofulven. Mol Cancer Ther, 5: 3153-3161, 2006.

38. Wang, W., Waters, S. J., MacDonald, J. R., Roth, C., Shentu, S., Freeman, J., Von Hoff, D. D., and Miller, A. R. Irofulven (6-hydroxymethylacylfulvene, MGI 114)induced apoptosis in human pancreatic cancer cells is mediated by ERK and JNK kinases. Anticancer Res, 22: 559-564, 2002.

39. Wang, W., Waters, S. J., MacDonald, J. R., Von Hoff, D. D., Strodel, W. E., and Miller, A. R. Irofulven (6-hydroxymethylacylfulvene, MGI 114) induces caspase 8 and 9-mediated apoptosis in human pancreatic adenocarcinoma cells. Anticancer Res, 21: 1789-1794, 2001. 
40. Liang, H., Salinas, R. A., Leal, B. Z., Kosakowska-Cholody, T., Michejda, C. J., Waters, S. J., Herman, T. S., Woynarowski, J. M., and Woynarowska, B. A. Caspase-mediated apoptosis and caspase-independent cell death induced by irofulven in prostate cancer cells. Mol Cancer Ther, 3: 1385-1396, 2004.

41. Teitz, T., Wei, T., Valentine, M., Vanin, E., Grenet, J., Valentine, V., Behm, F., Look, A., Lahti, J., and Kidd, V. Caspase 8 is deleted or silenced preferentially in childhood neuroblastomas with amplification of MYCN. Nat Med, 6: 529-535, 2000.

42. Wiltshire, T., Senft, J., Wang, Y., Konat, G. W., Wenger, S. L., Reed, E., and Wang, W. BRCA1 Contributes to Cell Cycle Arrest and Chemoresistance in Response to the Anticancer Agent Irofulven. Mol Pharmacol, 71: 1051-1060, 2007.

43. Friesen, C., Herr, I., Krammer, P. H., and Debatin, K. M. Involvement of the CD95 (APO-1/FAS) receptor/ligand system in drug-induced apoptosis in leukemia cells. Nat Med, 2: 574-577, 1996.

44. Fulda, S., Los, M., Friesen, C., and Debatin, K. M. Chemosensitivity of solid tumor cells in vitro is related to activation of the CD95 system. International Journal of Cancer, 76: 105-114, 1998.

45. Fulda, S., Sieverts, H., Friesen, C., Herr, I., and Debatin, K.-M. The CD95 (APO1/Fas) System Mediates Drug-induced Apoptosis in Neuroblastoma Cells. Cancer Res, 57: 3823-3829, 1997. 
46. Mo, Y.-Y. and Beck, W. T. DNA Damage Signals Induction of Fas Ligand in Tumor Cells. Mol Pharmacol, 55: 216-222, 1999.

47. Muller, M., Strand, S., Hug, H., Heinemann, E.-M., Walczak, H., Hofmann, W. J., Stremmel, W., Krammer, P. H., and Galle, P. R. Drug-induced Apoptosis in Hepatoma Cells Is Mediated by the CD95 (APO-1/Fas) Receptor/Ligand System and Involves Activation of Wild-type p53. J. Clin. Invest., 99: 403-413, 1997.

48. Landowski, T. H., Gleason-Guzman, M. C., and Dalton, W. S. Selection for Drug Resistance Results in Resistance to Fas-Mediated Apoptosis. Blood, 89: 18541861, 1997.

49. Seki, K., Yoshikawa, H., Shiiki, K., Hamada, Y., Akamatsu, N., and Tasaka, K. Cisplatin (CDDP) specifically induces apoptosis via sequential activation of caspase-8, -3 and -6 in osteosarcoma. Cancer Chemother Pharmacol, 45: 199-206, 2000.

50. Lacour, S., Hammann, A., Wotawa, A., Corcos, L., Solary, E., and DimancheBoitrel, M.-T. Anticancer Agents Sensitize Tumor Cells to Tumor Necrosis Factor-related Apoptosis-inducing Ligand-mediated Caspase-8 Activation and Apoptosis. Cancer Res, 61: 1645-1651, 2001.

51. Micheau, O., Solary, E., Hammann, A., and Dimanche-Boitrel, M.-T. Fas Ligandindependent, FADD-mediated Activation of the Fas Death Pathway by Anticancer Drugs. J. Biol. Chem., 274: 7987-7992, 1999. 
52. Nowak, G. Protein Kinase C-alpha and ERK1/2 Mediate Mitochondrial Dysfunction, Decreases in Active $\mathrm{Na}+$ Transport, and Cisplatin-induced Apoptosis in Renal Cells. J. Biol. Chem., 277: 43377-43388, 2002.

53. Ishikawa, Y. and Kitamura, M. Anti-apoptotic effect of quercetin: Intervention in the JNK- and ERK-mediated apoptotic pathways. Kidney Int, 58: 1078-1087, 2000.

54. Kim, Y. K., Kim, H. J., Kwon, C. H., Kim, J. H., Woo, J. S., Jung, J. S., and Kim, J. M. Role of ERK activation in cisplatin-induced apoptosis in OK renal epithelial cells. Journal of Applied Toxicology, 25: 374-382, 2005.

55. Holmstrom, T., Schmitz, I., Soderstrom, T., Poukkula, M., Johnson, V., Chow, S., Krammer, P., and Eriksson, J. MAPK/ERK signaling in activated T cells inhibits CD95/Fas-mediated apoptosis downstream of DISC assembly. The EMBO Journal, 19: 5418-5428, 2000.

56. Wilson, D. J., Alessandrini, A., and Budd, R. C. MEK1 Activation Rescues Jurkat T Cells from Fas-Induced Apoptosis. Cellular Immunology, 194: 67-77, 1999. 


\section{Figure Legends}

Figure 1. Caspase 8 protects mouse embryo fibroblast cells from irofulven induced apoptosis. A. Western blot analysis was performed to verify the protein expression status of the caspase 8 -/- and +/+ MEFs. Actin serves as loading control. B. Induction of apoptosis was determined by ELISA measuring fragmentation of DNA. Caspase 8-/- and $+/+$ cells were treated with 5 and $10 \mu \mathrm{M}$ of irofulven for 24 hours. The relative apoptosis was calculated as fold change from untreated cells minus background. The mean and standard deviation of triplicate experiments are shown. TNF $\alpha$ serves as positive control for induction of the death receptor mediated apoptosis pathway. C. Clonogenic survival assay was performed in the caspase 8-/- and +/+ MEFs. Cells were treated with 1, 2 and 4 $\mu \mathrm{M}$ of irofulven for 24 hours before incubation in drug free media. The mean and standard deviation of triplicate experiments are presented.

Figure 2. Caspase 8 protects NB7 cells from irofulven induced apoptosis. A. Western blot analysis was performed to verify the protein expression status of the NB7- and NB7 + C8 neuroblastoma cells. Actin serves as loading control. B. Induction of apoptosis was determined by ELISA measuring fragmentation of DNA. NB7- and NB7+C8 cells were treated with 5 and $10 \mu \mathrm{M}$ of irofulven for 24 hours. The relative apoptosis was calculated as fold change from untreated cells minus background. The mean and standard deviation of triplicate experiments are shown. TNF $\alpha$ serves as positive control for induction of the death receptor mediated apoptosis pathway. C. Annexin V staining was performed to quantify induction of apoptosis. NB7- and NB7+C 8 cells were treated with 
20 and $\mu \mathrm{M}$ of irofulven for 18 hours followed by annexin $\mathrm{V}$ and PI staining. Representative histograms are shown for each sample. D. Graphical representation of the mean for triplicate experiments are shown. E. Clonogenic survival assay was performed in the NB7- and NB7+C8 neuroblastoma cells. Cells were treated with 3, 5 and $7 \mu \mathrm{M}$ of irofulven for 24 hours before incubation in drug free media. The mean and standard deviation of triplicate experiments are presented.

Figure 3. Erk is rapidly phosphorylated after treatment with irofulven and acts in a proapoptotic role. A. Western blot analysis was performed on whole cell lysates from NB7- and NB7+C8 cells using a pERK specific antibody. Cells were treated with $30 \mu \mathrm{M}$ of irofulven for the indicated times. ERK2 serves as loading control. B. Western blot of caspase 8 -/- and +/+ MEFs was done after treatment with 20 and $30 \mu \mathrm{M}$ irofulven for 2 and 4 hours. ERK2 serves as loading control. C. Short time course western blot after 30 $\mu \mathrm{M}$ treatment with irofulven in the caspase $8-/-$ and $+/+$ MEFs reveals an early activation followed by a decrease in both cells before an increase in caspase $8+/+$ cells. ERK2 serves as loading control. D. Induction of apoptosis was determined by ELISA measuring fragmentation of DNA. Caspase 8 -/- and $+/+$ MEFs were treated with 5 and $10 \mu \mathrm{M}$ irofulven with and without PD98059 and LY290042. Mean of triplicate experiments is shown. 
Figure 1. Caspase 8 protects mouse embryo fibroblast cells from irofulven induced apoptosis.

Figure 1A

\section{Caspase 8 MEF \\ $-/-\quad+/+$}

Caspase

Actin

Figure 1B

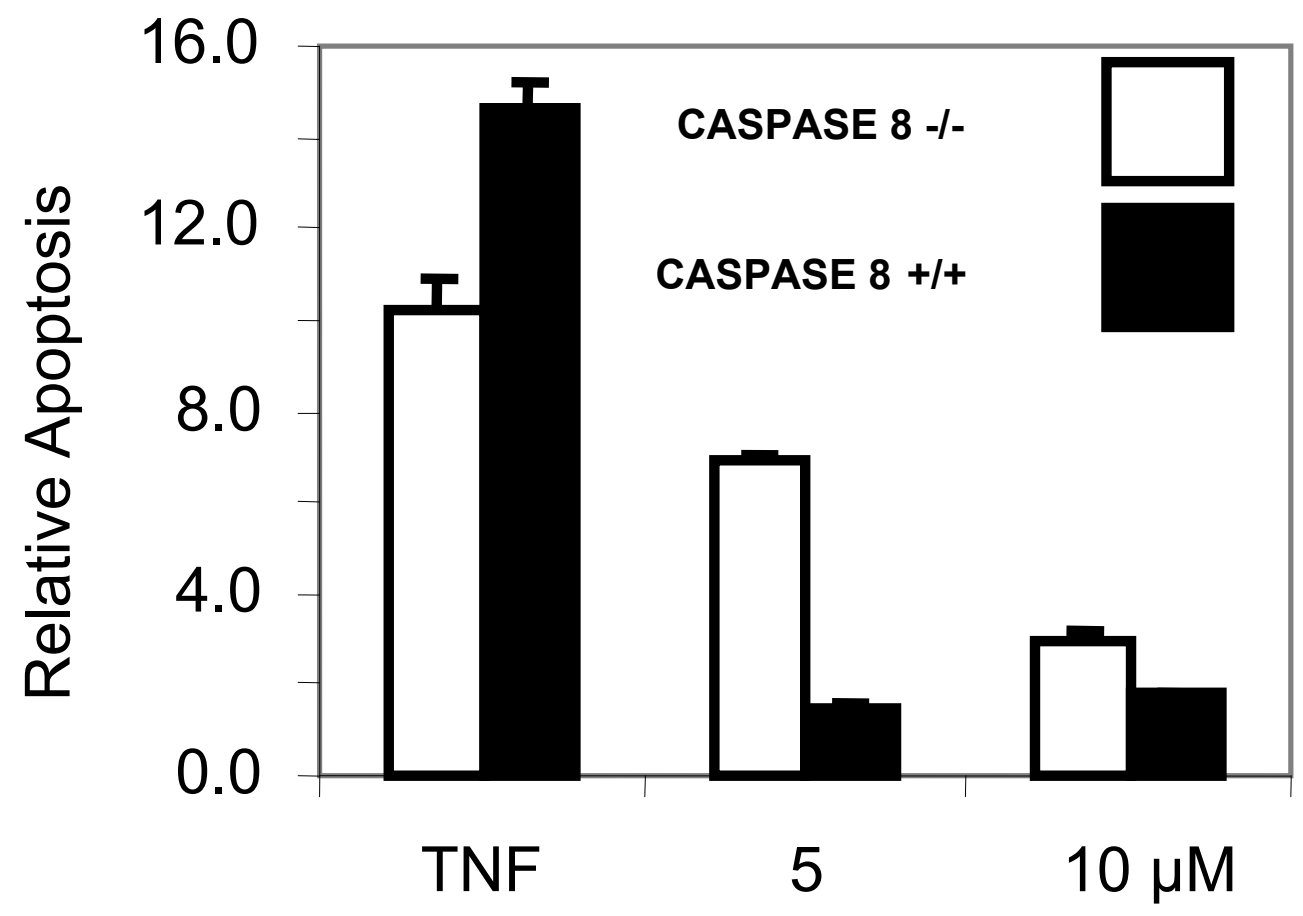


Figure 1C

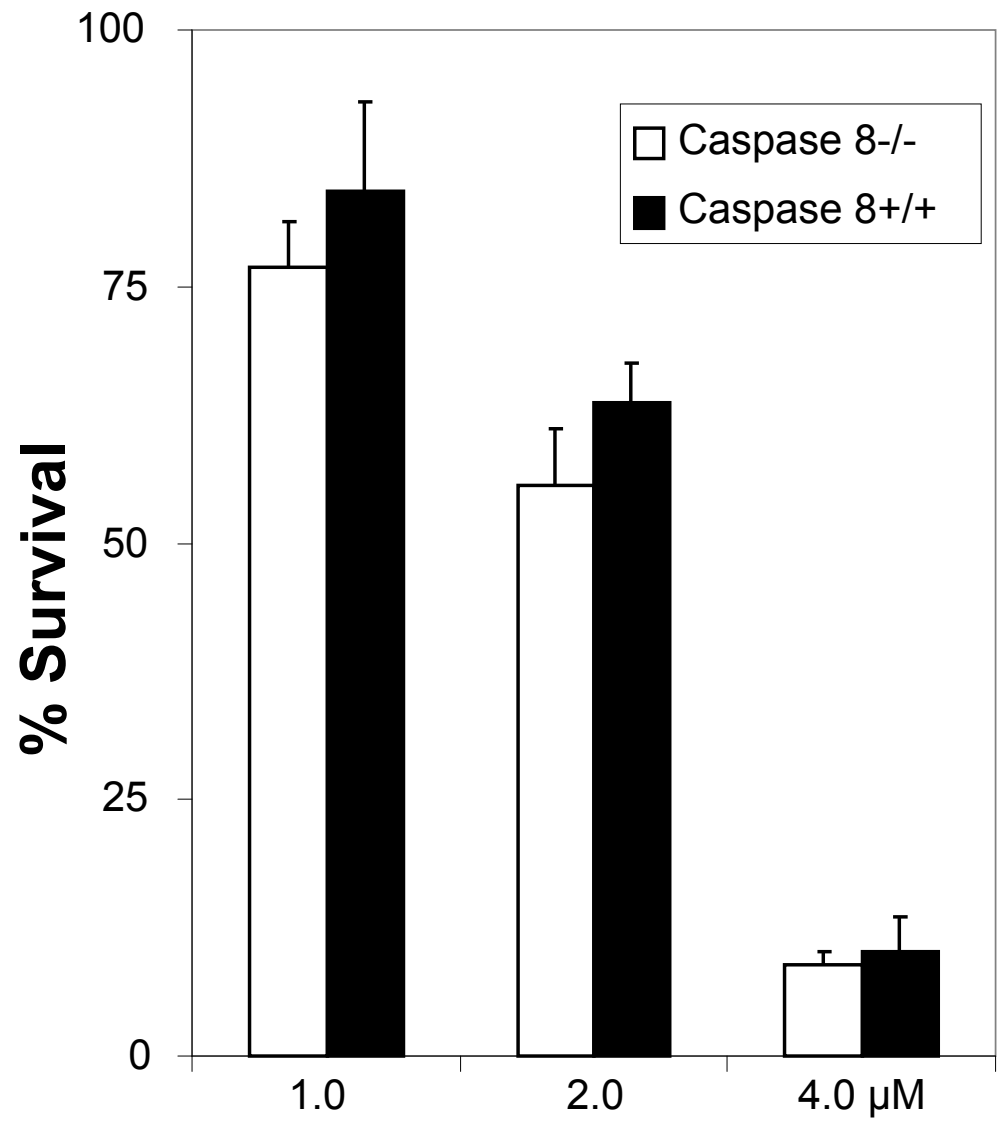


Figure 2. Caspase 8 protects NB7 cells from irofulven induced apoptosis.

Figure 2A

\title{
$\underline{\text { NB7 }}$
}

Caspase-8

$-\quad+\mathrm{C} 8$

Caspase 8

\author{
Actin
}

Figure 2B

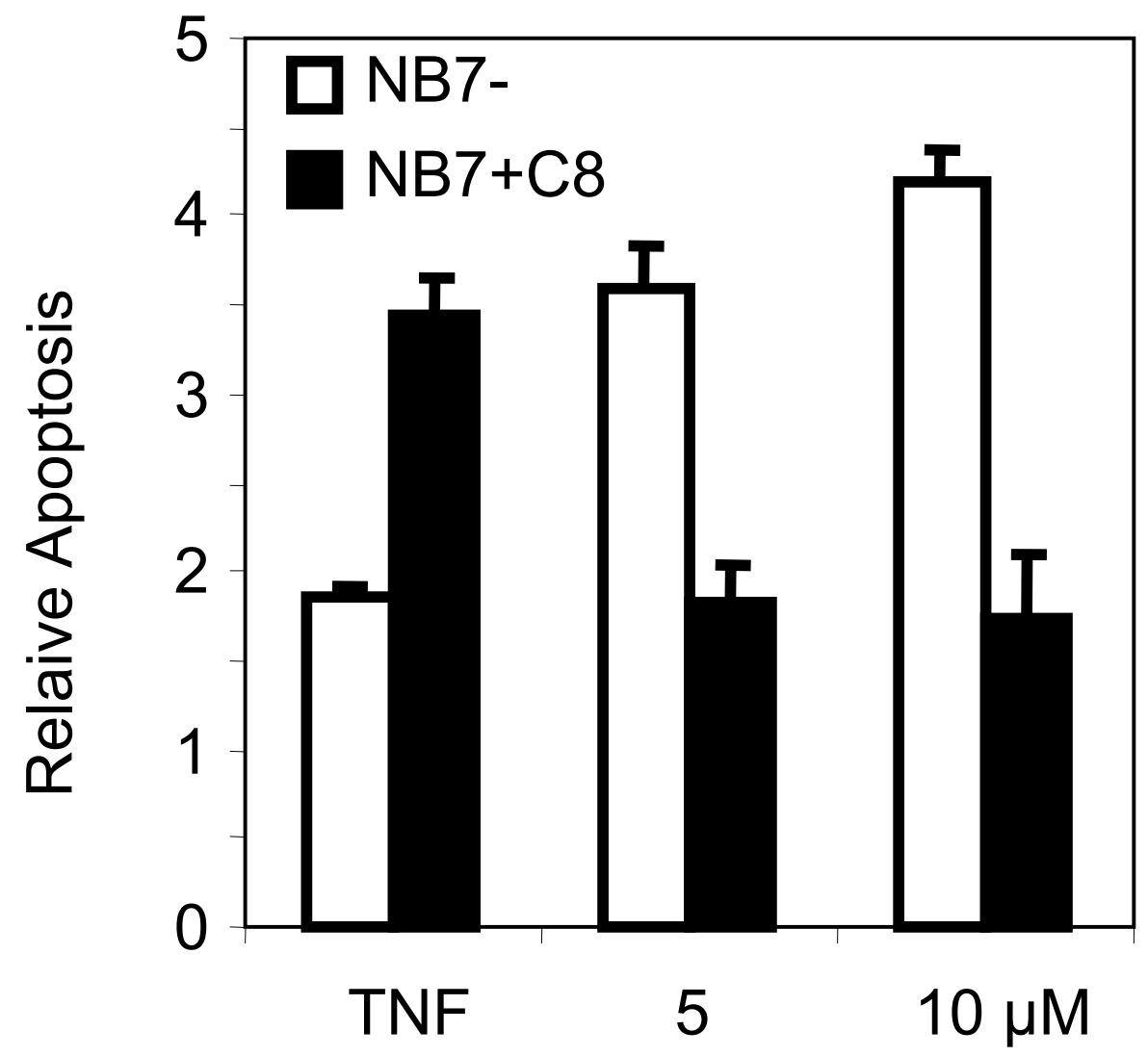




\section{Figure 2C}

\section{Annexin V NB7 -}
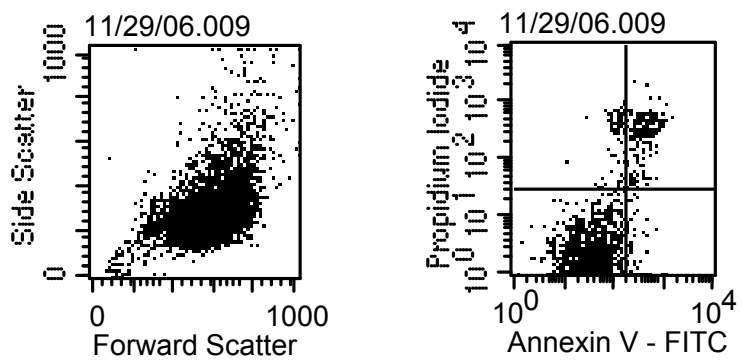

File: 11/29/06.009

\begin{tabular}{crrr} 
Quad & $\%$ Total & \\
\cline { 1 - 2 } UL & 2.11 & UNT \\
UR & 5.12 & \\
LL & 89.19 & \\
LR & 3.58 &
\end{tabular}
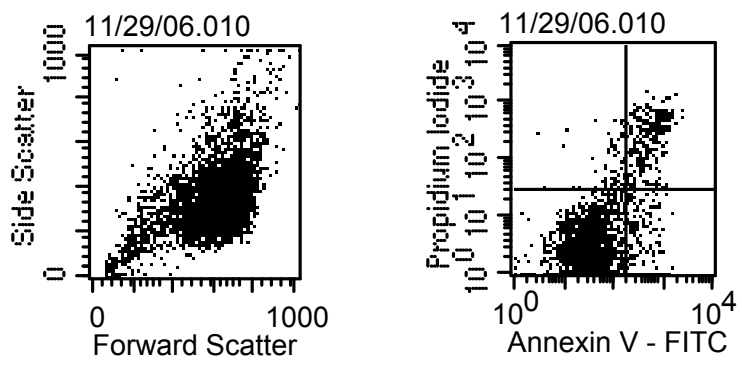

File: 11/29/06.010

Quad \% Total

$\begin{array}{rr}\text { UL } & 1.70 \\ \text { UR } & 6.58 \\ \text { LL } & 87.26 \\ \text { LR } & 4.46\end{array}$

TNF - 50nM
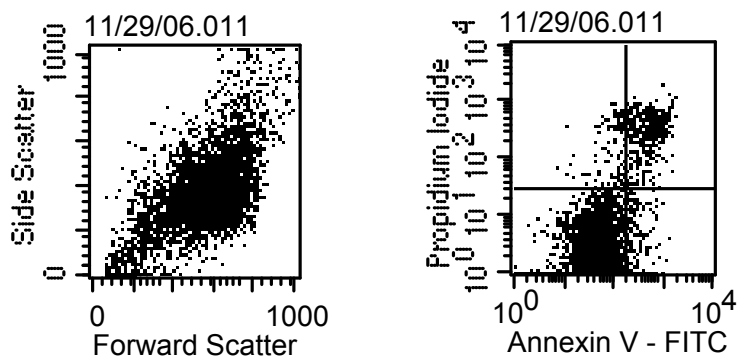

File: 11/29/06.011

\begin{tabular}{cr} 
Quad & $\%$ Total \\
\hline UL & 3.47 \\
UR & 10.26 \\
LL & 81.03 \\
LR & 5.24
\end{tabular}

$20 \mu \mathrm{M}-18 \mathrm{~h}$
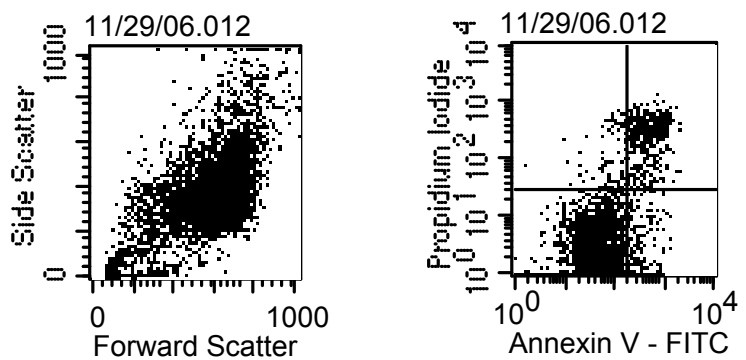

File: 11/29/06.012

\begin{tabular}{cr} 
Quad & $\%$ Tota \\
\hline UL & 2.78 \\
UR & 11.86 \\
LL & 79.48 \\
LR & 5.88
\end{tabular}

$30 \mu \mathrm{M}-18 \mathrm{~h}$ 
Figure 2C
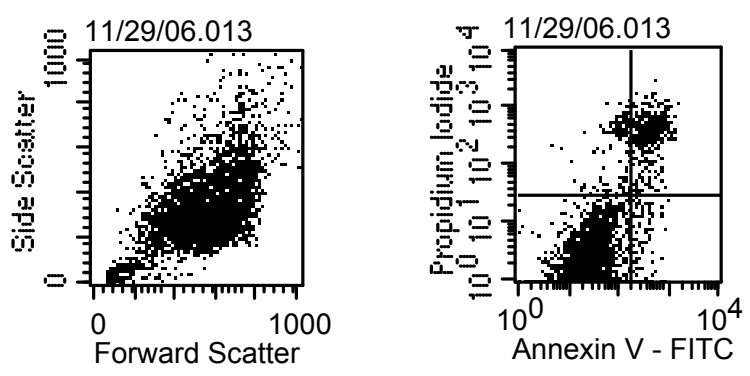

File: 11/29/06.013

\begin{tabular}{crrr} 
Quad & $\%$ Total & & UNT \\
\cline { 1 - 2 } UL & 4.77 & & \\
UR & 13.17 & \\
LL & 79.01 & \\
LR & 3.05 &
\end{tabular}
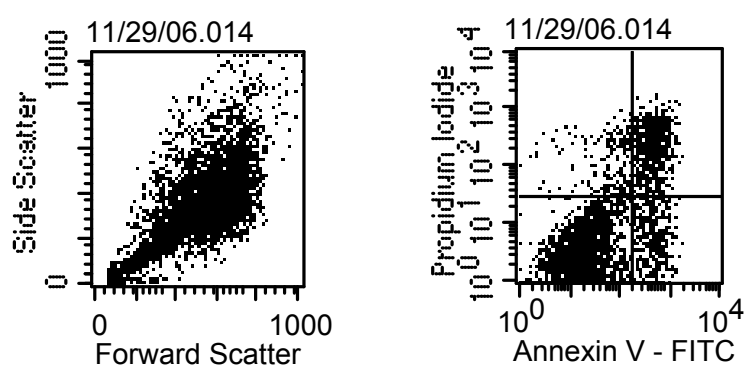

File: 11/29/06.014

\begin{tabular}{cr} 
Quad & $\%$ Total \\
\hline UL & 4.95 \\
UR & 14.83 \\
LL & 71.88 \\
LR & 8.35
\end{tabular}

TNF - 50nM
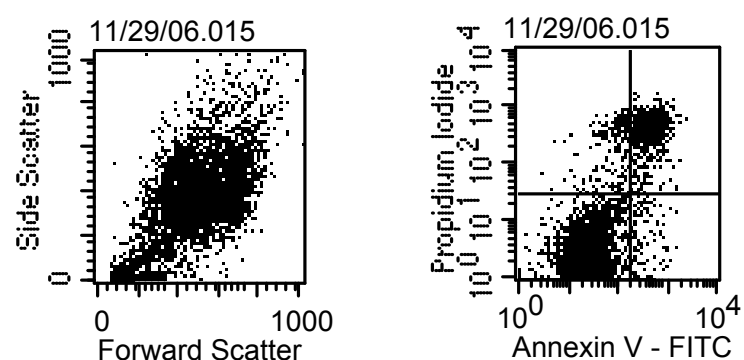

File: 11/29/06.015

Quad \% Total

$\begin{array}{rrrr}\text { UL } & 6.33 & 20 \mu \mathrm{M}-18 \mathrm{~h} \\ \text { UR } & 20.25 & \\ \text { LL } & 70.58 & \\ \text { LR } & 2.83 & \end{array}$
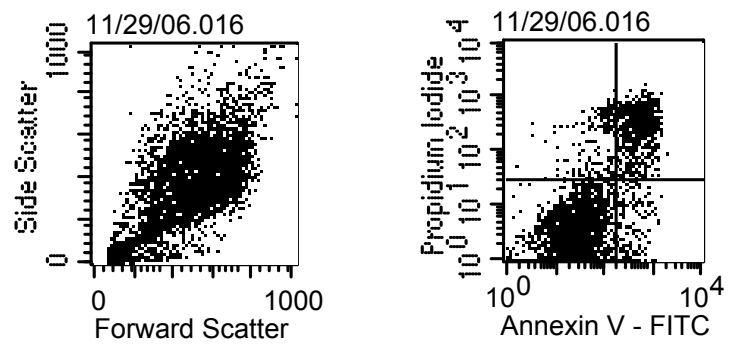

File: 11/29/06.016

\begin{tabular}{cr} 
Quad & $\%$ Total \\
\hline UL & 5.91 \\
UR & 20.38 \\
LL & 69.23 \\
LR & 4.49
\end{tabular}

$30 \mu \mathrm{M}-18 \mathrm{~h}$ 
Figure 2D

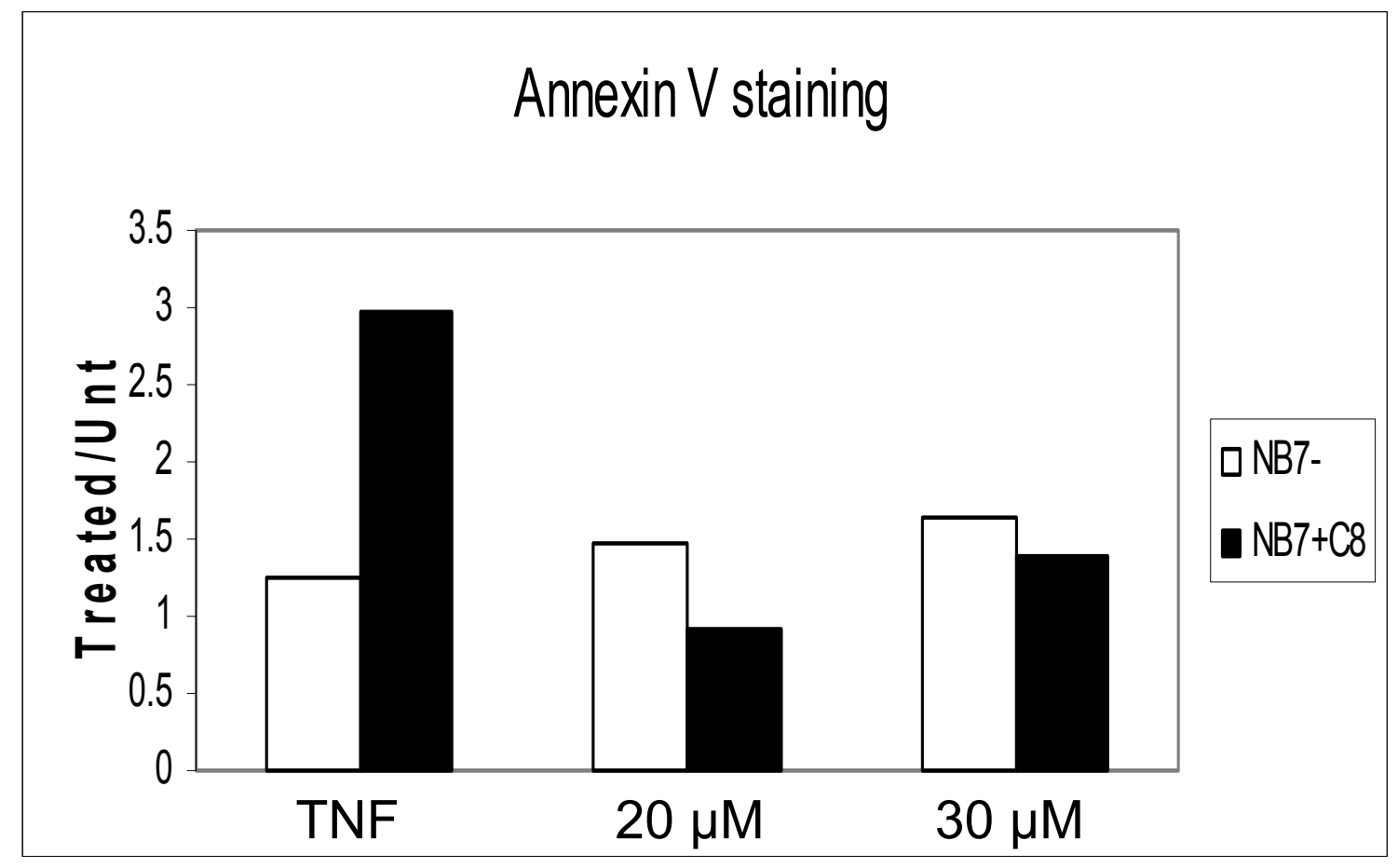


Figure 2E

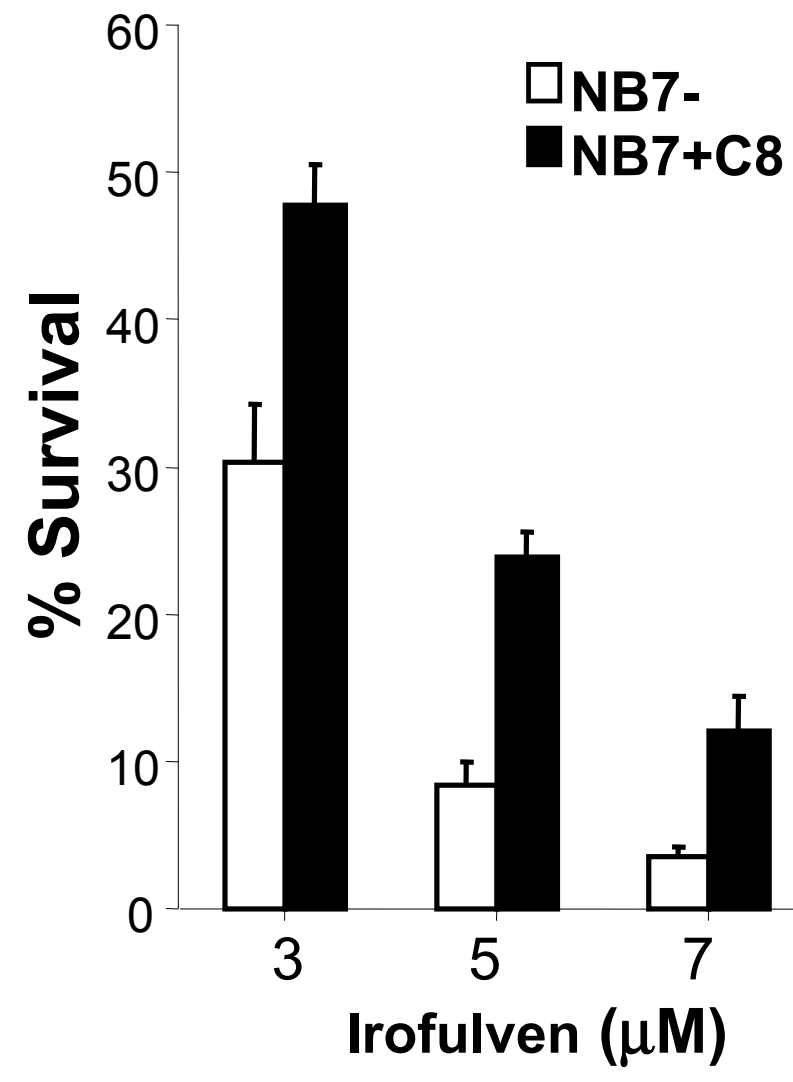


Figure 3. Erk is rapidly phosphorylated after treatment with irofulven and acts in a proapoptotic role.

Figure 3A

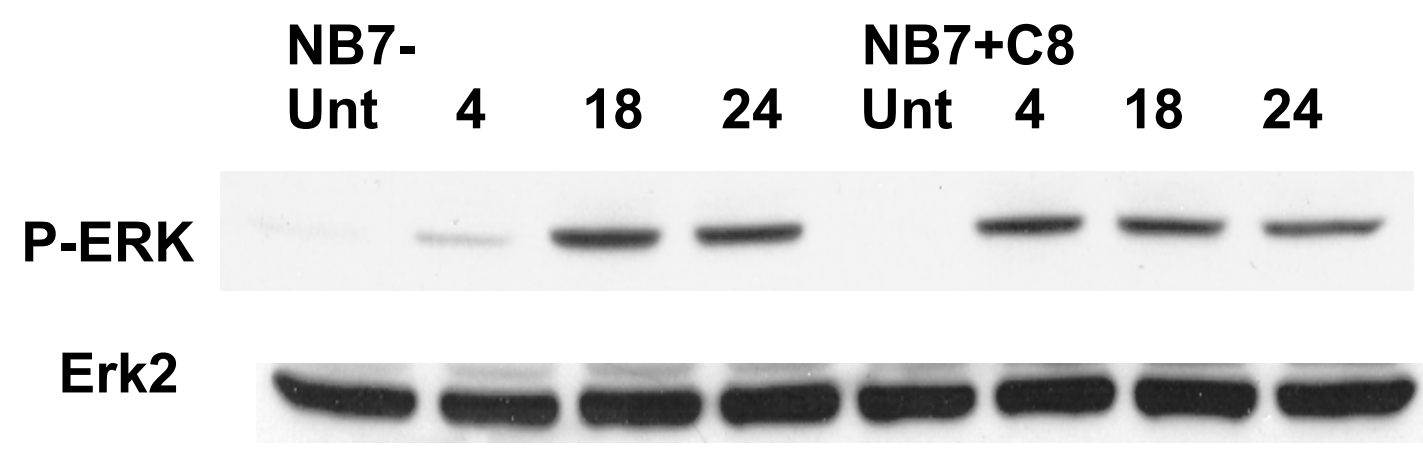

Figure 3B

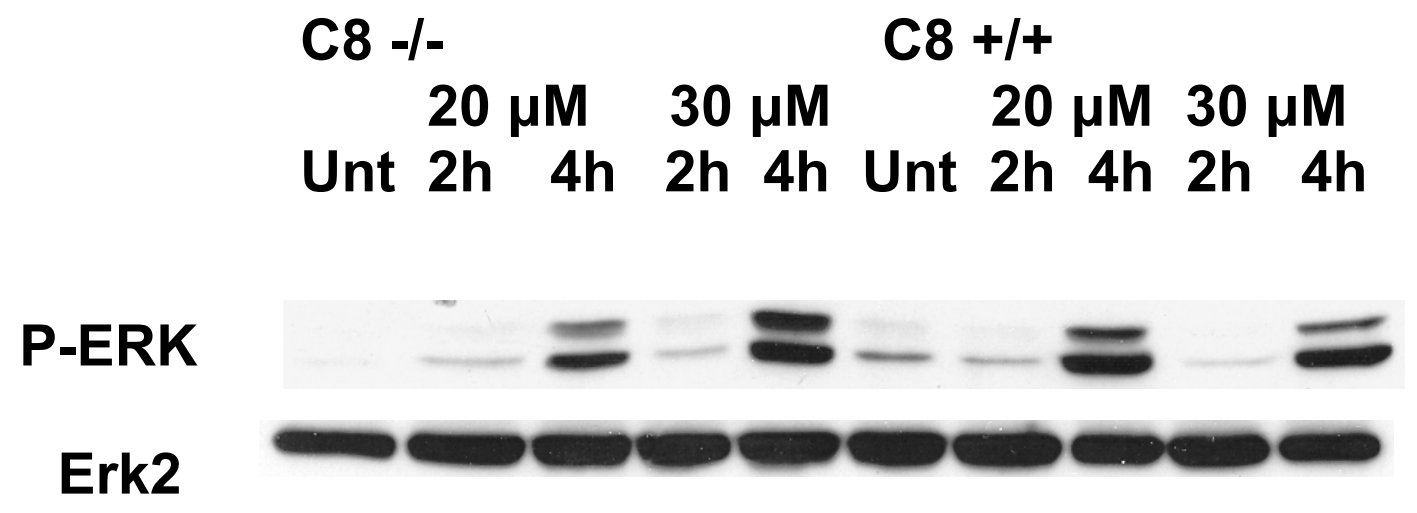


Figure 3C

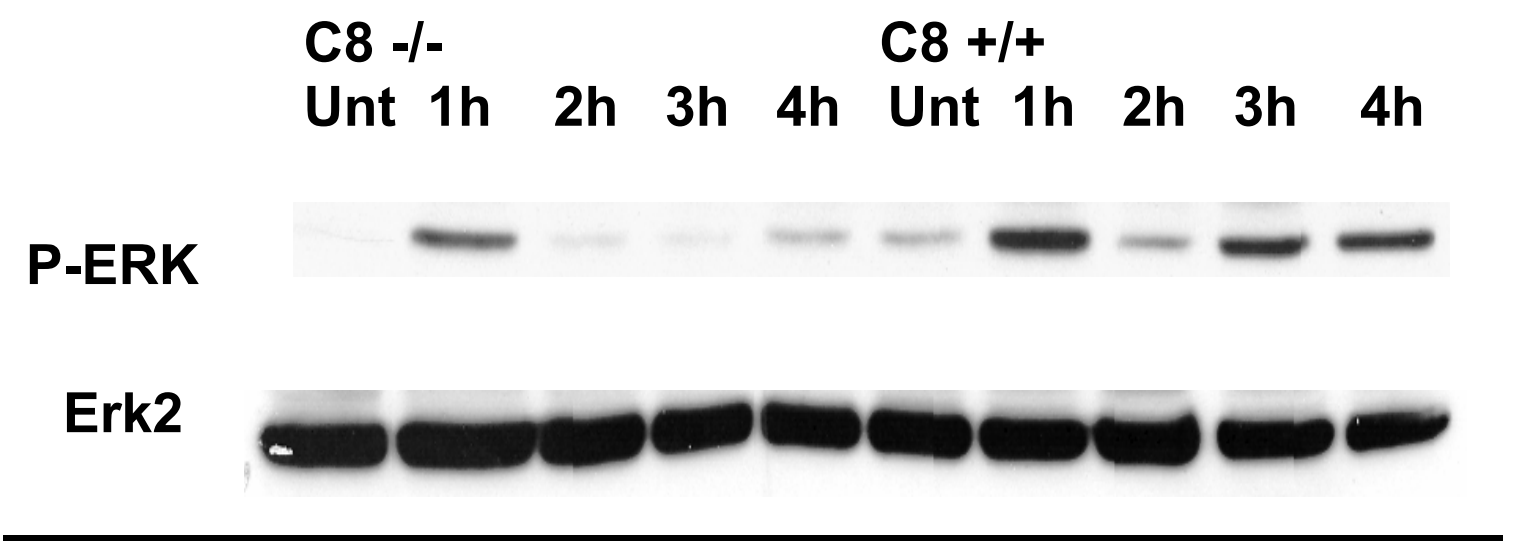


Figure 3D

Apoptosis ELISA

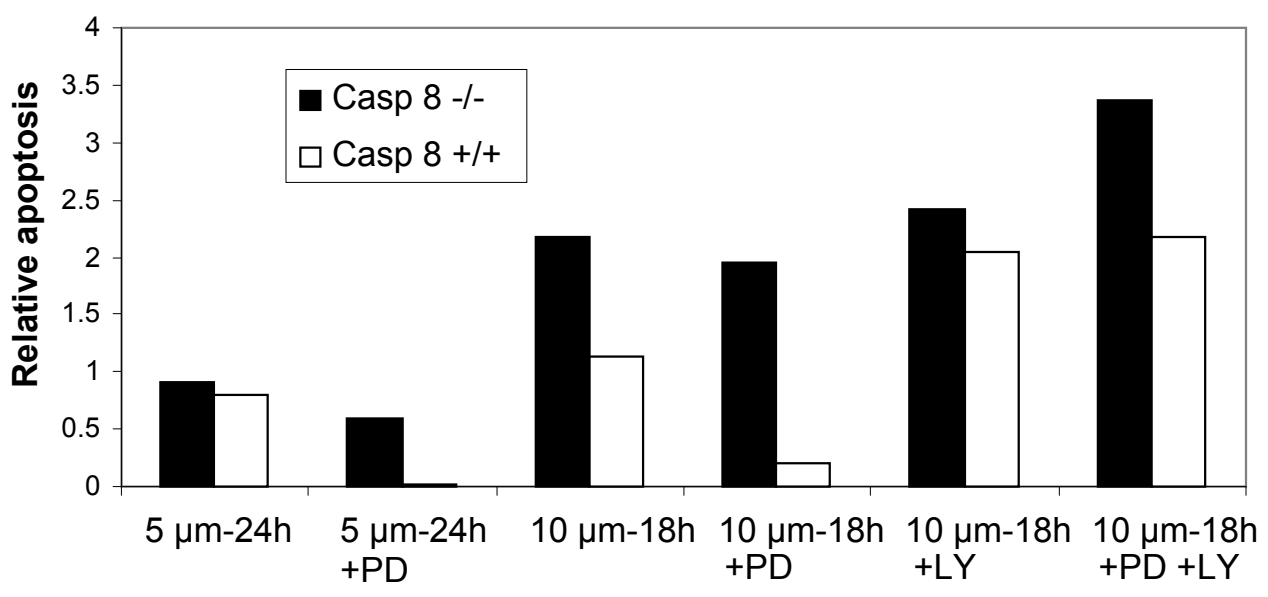




\section{Chapter V}

General Discussion 
The broad aim of our study was to determine the DNA damage response induced by irofulven. Specifically, we were interested in determining the role that BRCA1 played in this response and whether it would modulate chemosensitivity. We also sought to characterize the type of DNA damage caused by drug treatment. Lastly, we wanted to determine the involvement of caspase dependent apoptosis in response to irofulven.

The DNA damage response is a broad signaling network with the ability to sense damage to DNA and respond via multiple cellular mechanisms to ensure genomic integrity and prevent harmful mutations. Coordination of the DNA damage response is a multi-faceted response involving damage sensors, cell cycle checkpoints, and DNA repair proteins (1). Cells are also programmed with the ability to induce apoptosis if DNA damage is not able to be repaired (2). While many proteins have been identified that participate in the DNA damage response, the coordination and exact mechanism of the response remains to be elucidated. It is however apparent that the response to DNA damage is crucial for safeguarding the genome and avoiding neoplasia $(3,4)$. It is also a crucial characteristic of inherited cancers that could be exploited for therapeutic benefit.

In this study, we examined the activation of the DNA damage response elicited by a novel anti-cancer agent, irofulven. We initiated this study based on a preliminary observation that irofulven was able to produce chromosome abberations, including breaks and multi-radial structures, in several ovarian cancer cell lines as well as a breast cancer cell line lacking BRCA1. The first natural step to characterize the response was to determine the activation of DNA damage checkpoints and their dependence on BRCA1. We were able to clearly demonstrate BRCA1 dependent activation of the S phase and 
G2/M checkpoints in an isogenic breast cancer model with and without BRCA1.

HCC1937 cells with reconstituted BRCA1 protein experienced a several fold decrease in DNA synthesis after treatment as compared to HCC1937 cells lacking BRCA1. We also showed that the G2/M checkpoint was activated in a BRCA1 dependent manner as measured by the number of cells entering mitosis. We used phospho-histone 3 , a mitosis specific marker, to show the immediate activation of the G2/M checkpoint after treatment with irofulven. HCC1937 cells lacking BRCA1 failed to arrest at the G2 checkpoint while their BRCA1 proficient counterpart arrested before entering mitosis.

After demonstrating the activation of DNA damage checkpoints, we determined that treatment of breast cancer cells with irofulven resulted in the formation of DSBs and that cells lacking BRCA1 were several-fold more sensitive to treatment. This is an important piece of data which links the accumulation of DSBs to the lack of the HR repair pathway in BRCA1 deficient cells. Cells lacking BRCA1 failed to assemble Rad51 containing nuclear foci which are essential for the HR repair process. We also used comet assay and pulse field gel electrophoresis to measure the accumulation of DSBs after drug treatment. We found that cells lacking BRCA1 function accumulated more DSBs over time as compared to the BRCA1 proficient cells. The data from these experiments solidified the concept that the lack of the HR repair process in BRCA1 deficient cells contributes to increased sensitivity.

To further determine what role BRCA1 may play in overall resistance to irofulven treatment, we performed clonogenic survival assay. This assay demonstrated that chemosensitivity was increased several fold when cells lacked functional BRCA1. This highlights the clinical relevance of the project in that women who harbor a BRCA1 
mutation may respond more robustly to treatment with irofulven while those with functional BRCA1 may be more resistant. Since BRCA1 plays an important role in response to DNA damage, an inherited loss or mutation could potentially be exploited for therapeutic gain $(5,6)$. Response to chemotherapeutics based on BRCA1 status raises an important question regarding clinical outcomes of patients who render BRCA1 mutations. BRCA1 has been shown to modulate response to different types of anticancer agents by either increasing sensitivity or resistance. Specifically, BRCA1 has been shown to increase resistance several fold to agents that cause DNA damage such as DSBs, while it increases sensitivity several fold to spindle poisons (7). Several studies have noted a modulation of sensitivity to DNA damaging agents based on BRCA1 status including the standard therapies of IR and cisplatin (7-13). Many times, these studies are able to link chemosensitivity to a specific function of BRCA1 such as DNA repair or checkpoint control. This type of response highlights the contrasting pathways that chemotherapeutics activate and emphasizes the need for individualized therapy based on status of important markers that include BRCA1. These findings could easily translate into a clinical study in which patient response is determined based on status of BRCA1 mutations. Since BRCA1 mutations are prevalent in early onset breast cancer, it would be feasible to gather data from phase 2 clinical trials of irofulven and sort the data based on a number of known mutations.

This study represents the first data that demonstrates the ability of irofulven to produce double strand breaks. This is an important fact based on the knowledge that the type of DNA lesion induced has a strong impact on the responding pathways. Chemotherapeutic agents such as cisplatin or mitomycin C cause interstrand crosslinks 
(ICLs) repaired by recombinational repair, either NHEJ, HR or a combination of both as well as NER (5). Patients with mutations in key repair proteins often display hypersensitivity to DNA damaging agents (14-16). This data also provides key mechanistic information and a rationale to explore whether other proteins involved in the repair of DSBs are able to modulate chemosensitivity to irofulven and other DNA damaging agents.

One such protein, FANCD2, belongs to the fanconi anemia family of proteins and is mutated in a rare genetic disorder leading to genomic instability and sensitivity to DNA cross-linking agents $(17,18)$. A large complex of fanconi anemia proteins is assembled at the sites of DSBs leading to activation of FANCD2 which has been shown to interact with BRCA1, BRCA2 and Rad51 as key components in regulation of HR repair (17-21). Concurrently with this project, our lab also demonstrated that chemosensitivity to irofulven could also be modulated by FANCD2 (22). This supports the notion that other proteins involved in the HR repair process are important for response to irofulven and could be used to predict sensitivity. These studies will add to our ability to provide individualized therapy based on important markers linked to specific mechanisms of action and therapeutic response. It also provides a reasonable basis to further study proteins involved in the HR repair pathway as potential modulators of chemosensitivity to irofulven.

Another aspect of the DNA damage response that we investigated was the induction of apoptosis after irofulven treatment. Our last aim was to determine the specific caspase dependent response to irofulven. We started by investigating the role that caspase 2 played in cell death after irofulven treatment. We determined that irofulven 
induced cell death was initiated by caspase 2 and was also mediated by the mitochondrial pathway. This is in line with much evidence that suggests the mitochondrial pathway as a key regulator of chemotherapy and ionizing radiation induced apoptosis (23-28). Caspase 2 is unique in that the structure and sequence homology are closely related to that of the other initiator caspases while the cleavage specificity is more closely related to executioner caspases 3 and 7 (29). Several studies have demonstrated a role for caspase 2 in initiating apoptosis upstream of the mitochondria and it appears to be required for induction of apoptosis triggered by DNA damage (30-35). Our results indicate an activation of apoptosis that is initiated by caspase 2 and subsequently mediated through the mitochondrial pathway. The mitochondrial controlled pathway is a key component in the induction of apoptosis in response to ionizing radiation and several chemotherapeutics $(23,26-28)$. Consistent with these studies, we found that the mitochondrial pathway played a role in apoptosis induced by irofulven. Importantly, we also noted that caspases 2 and 9 render cells more chemosensitive to irofulven. This is an important test that determines whether a specific protein could be used to predict therapeutic outcome.

How caspase 2 is activated by various stress stimuli is still poorly understood. It has been reported that caspase 2 activation occurs by forming complex with PIDD (p53induced protein with a death domain) and the adapter protein RAIDD (RIP(ribosomeinactivating protein)-associated ICH-1/CED-homologous protein with death domain). Increased PIDD expression may result in caspase 2 activation as a means to regulate apoptosis $(16,41)$. However, a recent study indicated that caspase 2 activation is independent of p53 in response to docetaxel-induced apoptosis (11). We have observed 
that irofulven induces p53 accumulation and activation $(31,34)$. However, we and others have conducted studies in tumor cell lines with wild-type, mutant or null p53; and demonstrated that the cytotoxicity induced by irofulven is independent of p53 status ( 34 , 42). Therefore, caspase 2 initiated apoptosis induced by irofulven may not be regulated by $\mathrm{p} 53$. Additional work is required to identify the potential proteins triggering caspase 2 activation as well as the link between DNA damage signaling and caspase 2 in response to irofulven.

The next goal in our investigation of irofulven induced apoptosis was to determine the role of the death receptor pathway. In this study, we demonstrated that caspase 8 activity protected both MEFs and NB7 cells from irofulven induced apoptosis. We also determined that cells lacking caspase 8 are more sensitive to treatment than their wild type counterpart. Numerous studies have demonstrated a role for mitochondrial controlled apoptosis in response to DNA damaging agents and ionizing radiation, but there is also evidence for involvement of the death receptor mediated pathway $(23,26-28$, 36-39). This led us to explore a role for caspase 8 in irofulven induced apoptosis.

Surprisingly, we found that caspase 8 seemed to offer protection from irofulven induced apoptosis. This represents a novel finding based on what we know about other DNA damaging agents.

We were also able to determine that the MAP kinase pathway, specifically ERK, was acting as a proapoptotic signal in both the MEF and NB7 sets of cells. We provided evidence that blocking the phosphorylation of ERK using the PD98059 MEK inhibitor we were able to significantly reduce induction of apoptosis. A question still remains as to 
whether this would modulate an increased survival overall or was a short term effect that could be compensated for via alternate pathways.

One of the more interesting observations we made was the apparent survival response we saw in irofulven treated cells with wild type caspase 8 function. An empirical observation was made while carrying out the experiments involving the PD98059 inhibitor in conjunction with irofulven treatment. We noticed that cells pretreated with PD98059 followed by addition of irofulven actually increased cell proliferation versus the untreated control group. Cells were plated at approximately $30 \%$ confluence before they were subjected to any type of treatment. Within 12-18 hours after cells were treated the control group had grown to approximately $60 \%$ confluence while the irofulven + PD98059 group had reached nearly $100 \%$ confluence. This was compared to the irofulven only group which was still around $30 \%$ confluency with a number of cells floating in the media. This experiment serves as a preliminary experiment that raises a number of new questions about the mechanism of action of irofulven. It strongly suggests the activation of a survival or proliferation signal that is somehow dependent on activity of caspase 8 . Elucidation of this signal represents the beginning of a new investigation which might help explain the protective effect we see when caspase 8 proficient cells are treated with irofulven.

We briefly looked at the possibility that the PI3 kinase pathway might serve as the survival signal by using the LY290042 inhibitor in conjunction with irofulven. Our preliminary results indicated that PI3 kinase is not acting as the survival signal, but our data was not thorough enough to draw a reliable conclusion. This opens the door to a 
number of experiments that could be carried out in order to determine if caspase 8 does indeed activate a survival response.

Collectively, the work discussed here has elucidated several aspects of the DNA damage response activated by irofulven. We have demonstrated the activation of both $\mathrm{S}$ and $\mathrm{G} 2 / \mathrm{M}$ cell cycle checkpoints in a BRCA1 dependent manner. We have illustrated that irofulven treatment results in the formation of double strand breaks and that BRCA1 helps to maintain chromosome integrity in response to irofulven. We have shown that the DSBs formed by irofulven treatment are repaired via homologous recombination and that cells that are deficient in the repair of DSBs are more chemosensitive. Furthermore, we have demonstrated that cell death after irofulven treatment in initiated in a caspase 2 dependent manner and also involves activation of the mitochondrial pathway. We have elucidated a mechanism whereby caspase 8 is able to protect from irofulven induced apoptosis and determined that ERK is involved as a proapoptotic signal. We have also noted the potential activation of a survival signal by irofulven that may serve as a mechanism for the increased chemoresistance we observed in caspase 8 proficient cells. Taken together, this work has provided insight into the mechanism of action of irofulven that can be used to individualize therapy as well as providing a foundation for further investigation into the signals activated by this novel DNA damaging agent. 


\section{References}

1. Zhou, B. B. and Elledge, S. J. The DNA damage response: putting checkpoints in perspective. Nature, 408: 433-439, 2000.

2. Clarke, A. R., Purdie, C. A., Harrison, D. J., Morris, R. G., Bird, C. C., Hooper, M. L., and Wyllie, A. H. Thymocyte apoptosis induced by p53-dependent and independent pathways. 362: 849-852, 1993.

3. Bartkova, J., Horejsi, Z., Koed, K., Kramer, A., Tort, F., Zieger, K., Guldberg, P., Sehested, M., Nesland, J. M., Lukas, C., Orntoft, T., Lukas, J., and Bartek, J. DNA damage response as a candidate anti-cancer barrier in early human tumorigenesis. 434: 864-870, 2005.

4. Hartwell, L. H. and Kastan, M. B. Cell cycle control and cancer. Science, 266: 1821-1828, 1994.

5. Hoeijmakers, J. H. Genome maintenance mechanisms for preventing cancer. Nature, 411: 366-374, 2001.

6. Farmer, H., McCabe, N., Lord, C. J., Tutt, A. N. J., Johnson, D. A., Richardson, T. B., Santarosa, M., Dillon, K. J., Hickson, I., Knights, C., Martin, N. M. B., Jackson, S. P., Smith, G. C. M., and Ashworth, A. Targeting the DNA repair defect in BRCA mutant cells as a therapeutic strategy. Nature, 434: 917-921, 2005. 
7. Quinn, J. E., Kennedy, R. D., Mullan, P. B., Gilmore, P. M., Carty, M., Johnston, P. G., and Harkin, D. P. BRCA1 functions as a differential modulator of chemotherapy-induced apoptosis. Cancer Res, 63: 6221-6228, 2003.

8. Abbott, D. W., Thompson, M. E., Robinson-Benion, C., Tomlinson, G., Jensen, R. A., and Holt, J. T. BRCA1 expression restores radiation resistance in BRCA1defective cancer cells through enhancement of transcription-coupled DNA repair. J Biol Chem, 274: 18808-18812, 1999.

9. Le Page, F., Randrianarison, V., Marot, D., Cabannes, J., Perricaudet, M., Feunteun, J., and Sarasin, A. BRCA1 and BRCA2 are necessary for the transcription-coupled repair of the oxidative 8-oxoguanine lesion in human cells. Cancer Res, 60: 5548-5552, 2000.

10. Taniguchi, T., Tischkowitz, M., Ameziane, N., Hodgson, S. V., Mathew, C. G., Joenje, H., Mok, S. C., and D'Andrea, A. D. Disruption of the Fanconi anemiaBRCA pathway in cisplatin-sensitive ovarian tumors. Nat Med, 9: 568-574, 2003.

11. Wiltshire, T., Senft, J., Wang, Y., Konat, G. W., Wenger, S. L., Reed, E., and WANG, W. BRCA1 Contributes to Cell Cycle Arrest and Chemoresistance in Response to Anticancer Agent, Irofulven. Mol Pharmacol mol.106.029504, 2007.

12. Tassone, P., Tagliaferri, P., Perricelli, A., Blotta, S., Quaresima, B., Martelli, M., Goel, A., Barbieri, V., Costanzo, F., Boland, C., and Venuta, S. BRCA1 expression modulates chemosensitivity of BRCA1-defective HCC1937 human breast cancer cells. Br J Cancer, 88: 1285-1291, 2003. 
13. Fedier, A., Steiner, R., Schwarz, V., Lenherr, L., Haller, U., and Fink, D. The effect of loss of Brcal on the sensitivity to anticancer agents in p53-deficient cells. Int J Cancer, 22: 1169-1173, 2003.

14. Powell, S. N. and Kachnic, L. A. Roles of BRCA1 and BRCA2 in homologous recombination, DNA replication fidelity and the cellular response to ionizing radiation. Oncogene, 22: 5784-5791, 2003.

15. Chen, C. F., Chen, P. L., Zhong, Q., Sharp, Z. D., and Lee, W. H. Expression of BRC repeats in breast cancer cells disrupts the BRCA2-Rad51 complex and leads to radiation hypersensitivity and loss of G(2)/M checkpoint control. J Biol Chem, 274: 32931-32935, 1999.

16. Xia, F., Taghian, D. G., DeFrank, J. S., Zeng, Z.-C., Willers, H., Iliakis, G., and Powell, S. N. Deficiency of human BRCA2 leads to impaired homologous recombination but maintains normal nonhomologous end joining. PNAS, 98: 8644-8649, 2001.

17. Venkitaraman, A. R. Tracing the network connecting BRCA and Fanconi anaemia proteins. Nat Rev Cancer, 4: 266-276, 2004.

18. D'Andrea, A. D. and Grompe, M. The Fanconi anaemia/BRCA pathway. Nat Rev Cancer, 3: 23-34, 2003.

19. Garcia-Higuera, I., Taniguchi, T., Ganesan, S., Meyn, M. S., Timmers, C., Hejna, J., Grompe, M., and D'Andrea, A. D. Interaction of the Fanconi anemia proteins and BRCA1 in a common pathway. Mol Cell, 7: 249-262, 2001. 
20. Wang, X., Andreassen, P. R., and D'Andrea, A. D. Functional interaction of monoubiquitinated FANCD2 and BRCA2/FANCD1 in chromatin. Mol Cell Biol, 24: 5850-5862, 2004.

21. Hussain, S., Wilson, J. B., Medhurst, A. L., Hejna, J., Witt, E., Ananth, S., Davies, A., Masson, J. Y., Moses, R., West, S. C., De Winter, J. P., Ashworth, A., Jones, N. J., and Mathew, C. G. Direct interaction of FANCD2 with BRCA2 in DNA damage response pathways. Hum Mol Genet, 2004.

22. Wang, Y., Wiltshire, T., Senft, J., Wenger, S. L., Reed, E., and Wang, W. Fanconi anemia D2 protein confers chemoresistance in response to the anticancer agent, irofulven. Mol Cancer Ther, 5: 3153-3161, 2006.

23. Thornberry, N. A. and Lazebnik, Y. Caspases: Enemies Within. Science, 281: 1312-1316, 1998.

24. Green, D. R. Apoptotic pathways: paper wraps stone blunts scissors. Cell, 102: 1$4,2000$.

25. Roth, W. and Reed, J. C. Apoptosis and cancer: when BAX is TRAILing away. Nat Med, 8: 216-218, 2002.

26. Yoshida, H., Kong, Y.-Y., Yoshida, R., Elia, A. J., Hakem, A., Hakem, R., Penninger, J. M., and Mak, T. W. Apaf1 Is Required for Mitochondrial Pathways of Apoptosis and Brain Development. Cell, 94: 739-750, 1998. 
27. Hakem, R., Hakem, A., Duncan, G. S., Henderson, J. T., Woo, M., Soengas, M. S., Elia, A., de la Pompa, J. L., Kagi, D., Khoo, W., Potter, J., Yoshida, R., Kaufman, S. A., Lowe, S. W., Penninger, J. M., and Mak, T. W. Differential Requirement for Caspase 9 in Apoptotic Pathways In Vivo. Cell, 94: 339-352, 1998.

28. Soengas, M. S., Alarcon, R. M., Yoshida, H., Giaccia, A. J., Hakem, R., Mak, T. W., and Lowe, S. W. Apaf-1 and Caspase-9 in p53-Dependent Apoptosis and Tumor Inhibition. Science, 284: 156-159, 1999.

29. Zhivotovsky, B. and Orrenius, S. Caspase-2 function in response to DNA damage. Biochemical and Biophysical Research Communications, 331: 859-867, 2005.

30. Paroni, G., Henderson, C., Schneider, C., and Brancolini, C. Caspase-2-induced apoptosis is dependent on caspase-9, but its processing during UV- or tumor necrosis factor-dependent cell death requires caspase-3. J Biol Chem, 276: 21907$21915,2001$.

31. Guo, Y., Srinivasula, S. M., Druilhe, A., Fernandes-Alnemri, T., and Alnemri, E. S. Caspase-2 Induces Apoptosis by Releasing Proapoptotic Proteins from Mitochondria. J. Biol. Chem., 277: 13430-13437, 2002.

32. Robertson, J. D., Enoksson, M., Suomela, M., Zhivotovsky, B., and Orrenius, S. Caspase-2 Acts Upstream of Mitochondria to Promote Cytochrome c Release during Etoposide-induced Apoptosis. J. Biol. Chem., 277: 29803-29809, 2002. 
33. Mhaidat, N. M., Wang, Y., Kiejda, K. A., Zhang, X. D., and Hersey, P. Docetaxel-induced apoptosis in melanoma cells is dependent on activation of caspase-2. Mol Cancer Ther, 6: 752-761, 2007.

34. Lassus, P., Opitz-Araya, X., and Lazebnik, Y. Requirement for caspase-2 in stress-induced apoptosis before mitochondrial permeabilization. Science, 297: 1352-1354, 2002.

35. Tinel, A. and Tschopp, J. The PIDDosome, a Protein Complex Implicated in Activation of Caspase-2 in Response to Genotoxic Stress. Science, 304: 843-846, 2004.

36. Fulda, S., Los, M., Friesen, C., and Debatin, K. M. Chemosensitivity of solid tumor cells in vitro is related to activation of the CD95 system. International Journal of Cancer, 76: 105-114, 1998.

37. Fulda, S., Sieverts, H., Friesen, C., Herr, I., and Debatin, K.-M. The CD95 (APO1/Fas) System Mediates Drug-induced Apoptosis in Neuroblastoma Cells. Cancer Res, 57: 3823-3829, 1997.

38. Lacour, S., Hammann, A., Wotawa, A., Corcos, L., Solary, E., and DimancheBoitrel, M.-T. Anticancer Agents Sensitize Tumor Cells to Tumor Necrosis Factor-related Apoptosis-inducing Ligand-mediated Caspase-8 Activation and Apoptosis. Cancer Res, 61: 1645-1651, 2001. 
39. Micheau, O., Solary, E., Hammann, A., and Dimanche-Boitrel, M.-T. Fas Ligandindependent, FADD-mediated Activation of the Fas Death Pathway by Anticancer Drugs. J. Biol. Chem., 274: 7987-7992, 1999. 


\section{Appendix 1}

Fanconi anemia D2 protein confers chemoresistance in response to anticancer agent, irofulven

This manuscript was published in Molecular Cancer Therapeutics 


\section{Fanconi anemia D2 protein confers chemoresistance in response to the anticancer agent, irofulven}

\author{
Yutian Wang, ${ }^{1}$ Timothy Wiltshire, ${ }^{2}$ Jamie Senft, \\ Sharon L. Wenger, ${ }^{3}$ Eddie Reed, ${ }^{1,2}$ \\ and Weixin Wang ${ }^{1,2}$ \\ ${ }^{1}$ Mary Babb Randolph Cancer Center, ${ }^{2}$ Department of \\ Microbiology, Immunology, and Cell Biology, and ${ }^{3}$ Department \\ of Pathology, West Virginia University School of Medicine, \\ Morgantown, West Virginia
}

\begin{abstract}
The Fanconi anemia-BRCA pathway of genes are frequently mutated or epigenetically repressed in human cancer. The proteins of this pathway play pivotal roles in DNA damage signaling and repair. Irofulven is one of a new class of anticancer agents that are analogues of mushroom-derived illudin toxins. Preclinical studies and clinical trials have shown that irofulven is effective against several tumor cell types. The exact nature of irofulven-induced DNA damage is not completely understood. Previously, we have shown that irofulven activates $A T M$ and its targets, NBS1, SMC1, CHK2, and p53. In this study, we hypothesize that irofulven induces DNA double-strand breaks and FANCD2 may play an important role in modulating cellular responses and chemosensitivity in response to irofulven treatment. By using cells that are proficient or deficient for FANCD2, ATR, or ATM, we showed that irofulven induces FANCD2 monoubiquitination and nuclear foci formation. ATR is important in mediating irofulven-induced FANCD2 monoubiquitination. Furthermore, we showed that FANCD2 plays a critical role in maintaining chromosome integrity and modulating chemosensitivity in response to irofulven-induced DNA damage. Therefore, this study suggests that it might be clinically significant to target irofulven therapy to cancers defective for proteins of the Fanconi anemia-BRCA pathway. [Mol Cancer Ther $2006 ; 5(12): 3153-61]$
\end{abstract}

Received 7/20/06; revised 9/28/06; accepted 11/1/06 Grant support: National Cancer Institute grant 5R03CA107979 (W. Wang). The costs of publication of this article were defrayed in part by the payment of page charges. This article must therefore be hereby marked paynertisement in accordance with 18 U.S.C. Section 1734 solely to indicate this fact.

Note: Current address for E. Reed: Centers for Disease Control and Prevention, Atlanta, Georgia.

Requests for reprints: Weixin Wang, Mary Babb Randolph Cancer Center, West Virginia University, 1835 Health Sciences South, P.O. Box 9300 Morgantown, WV 26506. Phone: 304-293-2243; Fax: 304-293-4667. E-mail: wwang@hsc.wvu.edu

Copyright $\odot 2006$ American Association for Cancer Research. doi:10.1158/1535-7163.MCT-06-0427

\section{Introduction}

Fanconi anemia is a genetic cancer-susceptibility syndrome characterized by congenital abnormalities, bone marrow failure, and cellular sensitivity to DNA crosslinking agents $(1,2)$. There are at least 12 Fanconi anemia complementation groups (A, B, C, D1, D2, E, F, G, I, J, L, and $\mathrm{M}$ ), and 10 Fanconi anemia genes (A, C, D1/BRCA2, D2, E, F, G, J, L, and M) have been cloned (1-8). All of the Fanconi anemia proteins function in a common pathway. Fanconi anemia proteins (A, B, C, E, F, G, L, and M) assemble in a nuclear complex that is required for monoubiquitination/activation of the downstream FANCD2 protein $(1,2,4,6,9-13)$. FANCL has been shown to possess the E3 ubiquitin ligase activity to monoubiquitinate FANCD2 at $\mathrm{Lys}^{561}$ (11). The deubiquitinating enzyme of FANCD2, USP1, has also been recently identified (14). FANCD1 was shown to be identical to BRCA2 (15). FANCD2 colocalizes with BRCA1 and $B R C A 2$, and BRCA1 is required for monoubiquitination and foci formation of FANCD2 in response to DNA damage $(1,2,4,16-18)$. Therefore, BRCA1, BRCA2 (FANCD1), and Fanconi anemia proteins functionally merge in a common Fanconi anemia-BRCA pathway of DNA damage response $(1,2)$.

The Fanconi anemia-BRCA pathway of DNA damage response is frequently impaired in cancers due to genetic mutation or epigenetic repression $(1,2,4,9,10,19)$. BRCA1 and BRCA2 (FANCD1) are frequently mutated in familial breast and ovarian cancers $(>50 \%$; refs. $1,20,21)$. Germ line mutations in FANCD1 (BRCA2), FANCA, FANCC, or FANCG have been found in pancreatic cancer $(>10 \%$; refs. $22-27$ ). The BRCA2 (FANCD1) interacting protein, EMSY, which binds to BRCA2 and represses its function, was found to be amplified in sporadic breast (13\%) and highergrade ovarian $(17 \%)$ cancers $(28)$. FANCF, a protein upstream of FANCD2 and essential for its activation, is silenced by promoter methylation in several types of sporadic cancers including ovarian $(21 \%)$, breast $(17 \%)$, head and neck (15\%), non-small cell lung $(14 \%)$, and cervical $(30 \%)$ cancers $(19,29-31)$. The silencing of FANCF may be linked to acquired cisplatin resistance in a subset of ovarian cancers (31).

Irofulven (6-hydroxymethylacylfulvene, HMAF; MGI 114, NSC no. 683863) is a member of a new class of anticancer agents that are analogues of mushroom-derived illudin toxins. Preclinical studies and clinical trials have shown that irofulven is effective against several tumor cell types $(32-40)$. Thus far, the structure and nature of DNA damage caused by irofulven have not yet been fully characterized. Earlier studies suggested that the DNA damage caused by the illudin family of compounds might be repaired by the nucleotide excision repair pathway $(41,42)$. A recent study indicated that 
transcription-coupled nucleotide excision repair is the exclusive pathway in repairing illudin $\mathrm{S}$ and irofulvenelicited DNA lesions (43). However, in these studies, the homologous recombination pathway for DNA doublestrand break repair was not evaluated (41-43). Previously, we have shown that irofulven activates ATM and its targets, NBS1, SMC1, CHK2, and p53 (44). Recent reports indicate that ATM and CHK2 are specifically activated by drug (calicheamicin) or radiation-induced double-strand breaks $(45,46)$, and FANCD2 may play a role in homologous recombination pathway of double-strand break repair $(1,2)$. Therefore, we hypothesize that irofulven induces DNA double-strand breaks, and that FANCD2 may play an important role in maintaining chromosome stability and modulating chemosensitivity in response to irofulven.

To explore whether certain genetic defects in human cancer can be exploited to achieve preferential therapeutic outcomes by irofulven, we did this study to investigate the roles that FANCD2 might play in irofulven-induced DNA damage response. We showed that irofulven induces the monoubiquitination and foci formation of FANCD2. Irofulven-induced FANCD2 monoubiquitination is mediated by ATR. FANCD2 is critical for maintaining chromosome integrity and modulating chemosensitivity in response to irofulven.

\section{Materials and Methods \\ Cell Culture}

All cell lines were maintained in various media supplemented with $10 \%$ fetal bovine serum in a $37^{\circ} \mathrm{C}$ incubator with $5 \% \mathrm{CO}_{2}$ atmosphere. Human ovarian cancer cell lines A2780, CAOV3, and OVCAR3 were cultured in RPMI 1640. Human ovarian cancer cell line SKOV3 was cultured in McCoy's 5A medium. The vector and short hairpin FANCD2 (shFANCD2) stably transfected SKOV3 cells were cultured in McCoy's 5A medium containing $200 \mu \mathrm{g} / \mathrm{mL}$ of G418 (Invitrogen, Carlsbad, CA). The SV40-transformed Fanconi anemia D2 fibroblasts (PD20F) transfected with the vector, wild-type FANCD2, or K561R mutant FANCD2 (generously provided by Dr. Alan D'Andrea of Dana-Farber Cancer Institute, Harvard Medical School, Boston, MA; ref. 4) were grown in DMEM with $1 \mu \mathrm{g} / \mathrm{mL}$ of puromycin (Sigma, St. Louis, MO). The vector and ATM-transfected AT fibroblasts (AT22IJE-T-pEBS7 and AT22IJE-T-pEBS7-YZ5, generously provided by Dr. Yosef Shiloh, Tel Aviv University, Israel; ref. 47) were grown in DMEM with $100 \mu \mathrm{g} / \mathrm{mL}$ of hygromycin (Invitrogen). GM00847 human fibroblast expressing tetracycline-controlled, FLAG-tagged kinasedead ATR (generously provided by Drs. Stuart L. Schreiber and Shlomo Handeli of the Fred Hutchinson Cancer Research Center, Seattle, WA; ref. 48) was grown in DMEM supplemented with $400 \mu \mathrm{g} / \mathrm{mL}$ of $\mathrm{G} 418$ (Invitrogen). For FLAG-tagged kinase-dead ATR induction, cells were treated with $1.5 \mu \mathrm{g} / \mathrm{mL}$ of doxycycline (Sigma) for 48 hours. The Phoenix-ampho packaging cells (American Type Culture Collection, Manassas, VA) were grown in DMEM.

\section{Clonogenic Survival Assay}

To determine chemosensitivity and $\mathrm{IC}_{50}$ concentration, the clonogenic survival assay was done on $60 \mathrm{~mm}$ cell culture dishes as described previously (44). Cells were treated with different concentrations of irofulven for $1 \mathrm{~h}$, followed by drug-free incubation for $\sim 10$ days. Colonies were stained with crystal violet and counted if 50 or more cells were present. The $\mathrm{IC}_{50}$ concentration was calculated as the irofulven concentration that kills $50 \%$ of colonies of untreated controls.

\section{Western Blotting}

Western blotting was done as described previously (44). Total cellular extracts $(50 \mu \mathrm{g})$ were electrophoresed in SDS-PAGE gels. The primary antibodies used included: mouse anti-human actin (1:10,000 dilution), mouse anti-FLAG (1:200 dilution; Sigma), goat anti-human ATR (1:500 dilution), and mouse anti-human FANCD2 (1:500 dilution; Santa Cruz Biotechnology, Santa Cruz, CA). The secondary antibodies used were: sheep anti-mouse IgG-HRP (1:2,000 dilution; GE Healthcare Bio-Sciences Corp., Piscataway, NJ) and donkey anti-goat IgG-HRP (1:2,000 dilution; Santa Cruz Biotechnology). The relative amount of FANCD2 protein bands was quantified by densitometry.

Immunofluorescent Staining

Cells were plated on cover-slips and treated with irofulven for $1 \mathrm{~h}$ followed by $12 \mathrm{~h}$ of drug-free incubation. Cells were then fixed and stained with mouse anti-human FANCD2 (1:200 dilution; Santa Cruz Biotechnology). After staining with Alexa-fluor 546-conjugated goat antimouse IgG secondary antibody (1:200 dilution; Invitrogen), slides were mounted with Vectashield mounting medium (Vector Laboratories, Burlingame, CA) containing $5 \mathrm{ng} / \mathrm{mL}$ of 4',6-diamidino-2-phenylindole. Staining images were captured with an Olympus Provis AX70 fluorescence microscope (Olympus, Melville, NY), and Spot digital camera and software (Diagnostic Instruments, Sterling Height, MI).

RNA Interference

To knock-down ATR, one pair of 65-nucleotide short hairpin ATR oligos containing the target sequence of AACCTCCGTGATGTTGCTTGA (49), and one pair of 65 -nucleotide short hairpin bacterial green fluorescence protein oligos containing the target sequence of TGGAAGCGTTCAACTAGCA (44) were synthesized. After annealing, the 65-bp double-strand short hairpin ATR and short hairpin GFP fragments were inserted into pSuper.retro.puro vector (OligoEngine, Seattle, WA) and transfected into the Phoenix-ampho packaging cells by calcium phosphate precipitation. The viral supernatants from $100 \mathrm{~mm}$ dishes were collected $48 \mathrm{~h}$ after transfection. The SKOV3 cells on a $100 \mathrm{~mm}$ dish were then infected with $1 \mathrm{~mL}$ of viral supernatant and $4 \mu \mathrm{L}$ (4 $\mathrm{mg} / \mathrm{mL}$ ) of polybrene (Sigma). The medium was replaced with fresh medium $24 \mathrm{~h}$ after infection. Cells were treated with irofulven $48 \mathrm{~h}$ after infection. To knock-down FANCD2, three pairs of 65-nucleotide 
shFANCD2 oligos containing target sequences of AAGGTTCGCCAGTTGGTGATG, AAGTCAGCTATTAGATATGAG, and AAGAAATAAGATTCGATCAGG were designed and synthesized. After annealing, these three 65-bp double-stranded shFANCD2 fragments were inserted into pSilencer 2.1-U6-neo vector (Ambion, Austin, TX) and transfected into SKOV3 cells with Fugene 6 (Roche Applied Science, Indianapolis, IN). The pSilencer 2.1-U6-neo vector containing the scrambled sequence (Ambion) was transfected as the nonspecific control. Stable cell lines were established by selecting in medium containing G418.

Metaphase Spread

Cells were treated with irofulven for $1 \mathrm{~h}$ followed by $24 \mathrm{~h}$ of drug-free incubation. Colcemid $(400 \mathrm{ng} / \mathrm{mL}$; Biosciences, La Jolla, CA) was added to the medium $4 \mathrm{~h}$ before harvesting. After trypsinization, cells were washed once with PBS. Cell pellets were resuspended in $75 \mathrm{mmol} / \mathrm{L}$ of $\mathrm{KCl}$ and incubated at $37^{\circ} \mathrm{C}$ for $8 \mathrm{~min}$. After centrifugation, cells were fixed using a 3:1 absolute methanol to glacial acetic acid ratio at $4{ }^{\circ} \mathrm{C}$ for $2 \mathrm{~h}$ and then washed twice with fixative. Cells were resuspended in fixative and dropped onto slides. Slides were air-dried at room temperature and stained with 5\% Gurr's Giemsa stain (Biomedical Specialties, Santa Monica, CA) for $7 \mathrm{~min}$. Slides were rinsed twice with distilled water and air-dried. The images were recorded by light microscopy using an Olympus Provis AX70 fluorescence microscope (Olympus), and the Spot digital camera and software (Diagnostic Instruments).

\section{Results}

FANCD2 Is Monoubiquitinated in Response to Irofulven Treatment

To explore the role that FANCD2 might play in irofulven-induced chemosensitivity, ovarian cancer cell lines A2780, CAOV3, SKOV3, and OVCAR3 were treated with irofulven, FANCD2 ubiquitination status was assessed by Western blot. The results showed that FANCD2 was monoubiquitinated, as indicated by the larger form of FANCD2 (FANCD2-L), in all of the ovarian cancer cell lines after irofulven treatment. Some constitutive FANCD2 monoubiquitination was observed in SKOV3 cells (Fig. 1A).

FANCD2 forms foci at DNA damage sites, which are dependent on monoubiquitination (1, 2, 4, 16-18). Reports have indicated that BRCA1 is required for FANCD2 monoubiquitination and foci formation in response to DNA damage $(4,18,50)$. The ovarian cancer cell line SKOV3 is known to harbor a functional BRCA1 (51). To confirm that FANCD2 is monoubiquitinated and activated in response to irofulven-induced DNA damage, SKOV3 cells were treated with irofulven, immunofluorescent staining results showed that FANCD2 forms distinct foci after irofulven treatment (Fig. 1B).

The monoubiquitination of FANCD2 after irofulven treatment was further confirmed in the vector, wild-type

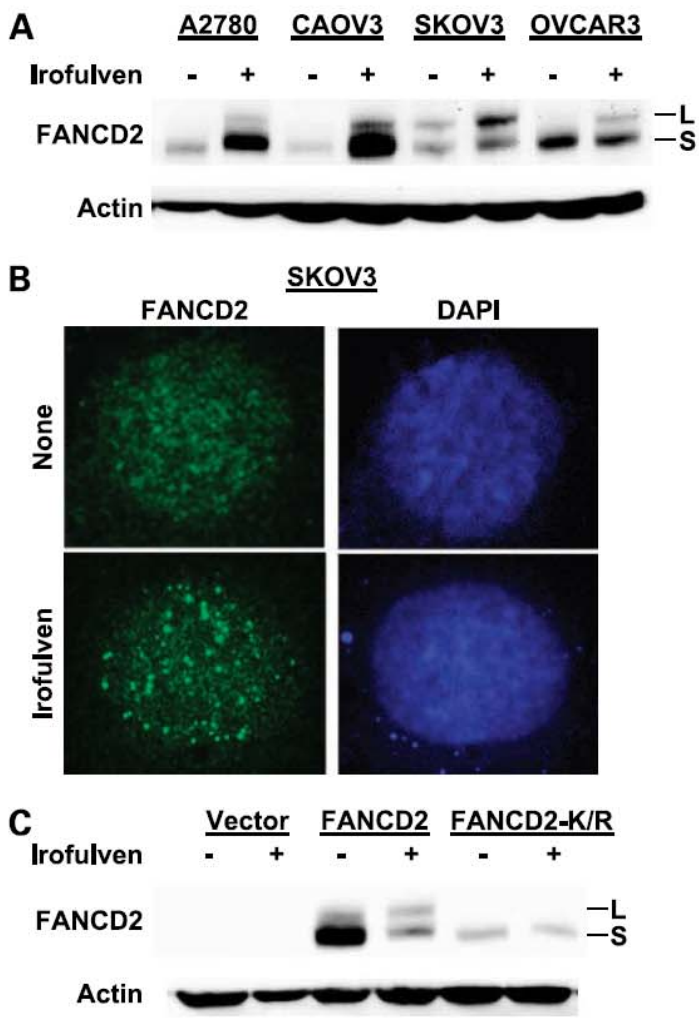

Figure 1. FANCD2 is monoubiquitinated after irofulven treatment. A, ovarian cancer cell lines A2780, CAOV3, SKOV3, and OVCAR3 were treated with $1 \times I C_{50}$ concentration of irofulven $(0.7,0.9,2.3$, and 1.0 Western blots were done with monoclonal antibodies against FANCD2 and actin. The original and monoubiquitinated forms of FANCD2 proteins are indicated as $\mathrm{S}$ and $\mathrm{L}$, respectively. B, immunofluorescent staining of FANCD2. SKOV3 cells were treated with $1 \times 1 \mathrm{C}_{50}$ concentration $(2.3 \mu \mathrm{mol} / \mathrm{L})$ of irofulven for $1 \mathrm{~h}$ followed by $12 \mathrm{~h}$ of drug-free incubation. Cells were fixed and stained hith fllowed by $12 \mathrm{~h}$ of drug-free incubation. Cells were fixed and stained indole. C, the vector, wild-type FANCD2, or K561R mutant FANCD2 transfected PD2OF fibroblasts were treated with $5 \mu \mathrm{mol} / \mathrm{L}$ of irofulven for $1 \mathrm{~h}$ followed by $24 \mathrm{~h}$ of drug-free incubation. Western blots were done with monoclonal antibodies against FANCD2 and actin. The original and monoubiquitinated forms of FANCD2 proteins are indicated as $\mathrm{S}$ and $\mathrm{L}$, respectively.

FANCD2 and K561R mutant (the monoubiquitination site Lys $^{561}$ was mutated) FANCD2-transfected FA-D2 fibroblasts (PD20F; ref. 4). Western blot results indicated that increased FANCD2 monoubiquitination was only observed in FANCD2-transfected cells. No monoubiquitinated FANCD2 was found in vector or K561R mutant FANCD2-transfected cells after irofulven treatment (Fig. 1C). Together, these results showed that FANCD2 is monoubiquitinated and activated following irofulven treatment. 
FANCD2 Monoubiquitination Is Mediated by ATR It has been reported that the phosphorylation of FANCD2 is mediated by ATM or ATR in response to IR or psoralen/UV-A $(52,53)$. The monoubiquitination of FANCD2 requires ATR, but not ATM, in response to IR, hydroxyurea, or mitomycin C (54). Thus far, the structure and nature of DNA damage caused by irofulven have not been fully characterized. We hypothesize that irofulven induces the generation of double-strand breaks based on the finding that irofulven activates ATM and its target

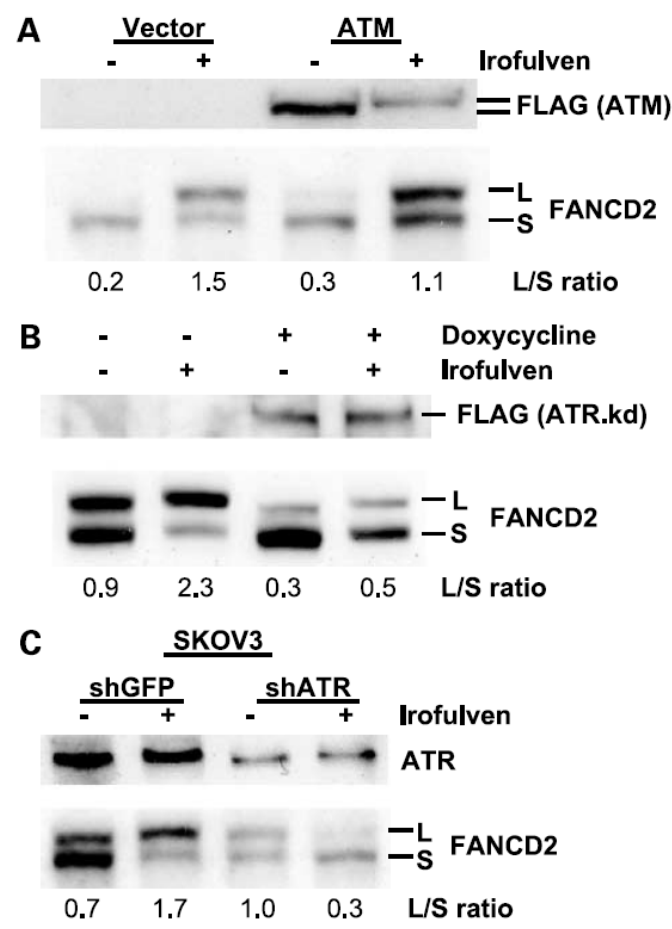

Figure 2. Irofulven-induced FANCD2 monoubiquitination was mediated by ATR. A, the vector and FLAG-tagged ATM-transfected AT fibroblasts (AT221JE-T-pEBS7 and AT22IJE-T-pEBS7-YZ5) were treated with $5 \mu \mathrm{mol} /$ (AT of irofulven for $1 \mathrm{~h}$ followed by $24 \mathrm{~h}$ of posttreatment incubation. Western blots were done with anti-FLAG and anti-FANCD2 antibodies. The ratio of FANCD2-L to FANCD2-S was determined by densitometry. B GM00847 human fibroblasts expressing tetracycline-controlled, FLAGtagged kinase-dead ATR were induced with doxycycline. The induced and uninduced cells were then treated with $5 \mu \mathrm{mol} / \mathrm{L}$ of irofulven for $1 \mathrm{~h}$ followed by $24 \mathrm{~h}$ of drug-free incubation. Western blots were done with anti-FLAG and anti-FANCD2 antibodies. The ratio of FANCD2 $L$ to FANCD2-S FANCD2-S was deterning by ders infected with viral supenatants containing short hairpin GFP or shot hairpin ATR, respectively. The short hairpin GFP was used as the nonspecific control. Forty-eight hours after infection, cells were treated with $2.3 \mu \mathrm{mol} / \mathrm{L}$ of irofulven for $1 \mathrm{~h}$ followed by $24 \mathrm{~h}$ of drug-free incubation. Western blots were done with anti-ATR and anti-FANCD2 antibodies. The ratio of FANCD2-L to FANCD2-S was determined by densitometry. genes NBS1, SMC1, and CHK2 (44). We therefore think it is important to determine whether irofulven-induced FANCD2 monoubiquitination is mediated by ATM or ATR. To examine whether ATM was involved in irofulven-induced FANCD2 monoubiquitination, the vector and FLAG-tagged ATM-transfected AT fibroblasts (AT22IJE-T-pEBS7 and AT22IJE-T-pEBS7-YZ5; ref. 47) were treated with irofulven. As shown in Fig. 2A, there was little difference in FANCD2 monoubiquitination between untreated cells. The expression of ATM, as indicated by Western blotting with anti-FLAG antibody, did not cause any further increase of FANCD2 monoubiquitination following irofulven treatment. Instead, the ratio of FANCD2-L (monoubiquitinated) to FANCD2-S (nonubiquitinated) was slightly decreased (from 1.5 to 1.1 ) between the vector and ATM-transfected AT cells following irofulven treatment. This suggests that ATM is not important for irofulven-induced FANCD2 monoubiquitination.

To test whether ATR was involved in irofulven-induced FANCD2 monoubiquitination, GM00847 human fibroblasts expressing tetracycline-controlled, FLAG-tagged kinasedead ATR (48) were used. Both doxycycline-induced and uninduced cells were treated with irofulven. As shown in Fig. 2B, the FLAG-tagged kinase-dead ATR was induced by doxycycline as determined by Western blot with anti-FLAG antibody. There was some constitutive FANCD2 monoubiquitination in untreated GM00847 cells. Upon irofulven treatment, there was an increase in FANCD2 monoubiquitination, the ratio of FANCD2-L to FANCD2-S increased from 0.9 to 2.3. When the kinase-dead ATR was induced by doxycycline in these cells, FANCD2 monoubiquitination was markedly blocked, the ratio of FANCD2-L to FANCD2-S was only slightly increased from 0.3 to 0.5 after irofulven treatment. Moreover, the overall FANCD2 monoubiquitination was also blocked. The ratio of FANCD2-L to FANCD2-S dropped from 0.9 to 0.3 in untreated cells; and from 2.3 to 0.5 in irofulven-treated cells (Fig. 2B).

To further confirm that ATR is important in mediating irofulven-induced FANCD2 monoubiquitination, an RNA interference approach was used to specifically knock-down the endogenous ATR expression in SKOV3 cells. The cells were infected with retrovirus containing short hairpin ATR or short hairpin GFP control construct, respectively. Western blot results indicated that the endogenous ATR level was knocked down by $>60 \%$ as quantified by densitometry (Fig. 2C). Some constitutive FANCD2 monoubiquitination was observed in SKOV3 cells. In short hairpin GFP control-infected cells, the ratio of FANCD2-L to FANCD2-S increased from 0.7 to 1.7 after irofulven treatment. In short hairpin ATRinfected cells, FANCD2 monoubiquitination was greatly blocked, the ratio of FANCD2-L to FANCD2-S was 1.0 in untreated cells and 0.3 in irofulven-treated cells (Fig. 2C). Taken together, these results indicate that irofulveninduced FANCD2 monoubiquitination is mediated by ATR. 
A

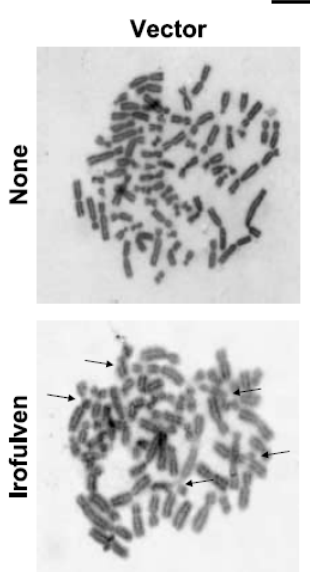

PD20F

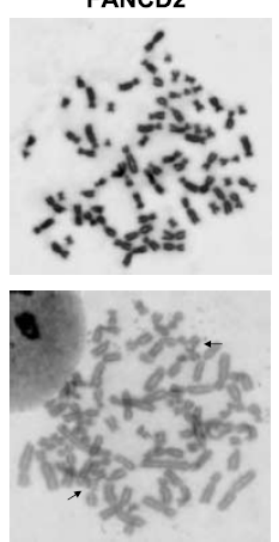

B

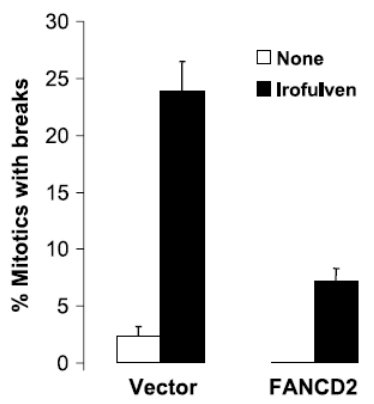

C

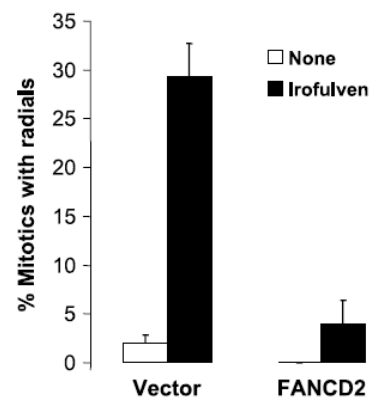

Figure 3. Chromosome aberrations induced by irofulven were related to FANCD2 status. A to $C$, the vector and wild-type FANCD2-transfected PD2OF cells were treated with $5 \mu \mathrm{mol} / \mathrm{L}$ of irofulven for $1 \mathrm{~h}$ followed by $24 \mathrm{~h}$ of drug-free incubation. Mitotic spread staining was done. Arrows, chromosome breaks and radials (A). Columns, the mean percentage of mitotic cells with four or more chromosome breaks (B) or with radials (C) from triplicate counts of 50 cells; bars, SD.

FANCD2 Is Important for Chromosome Integrity in Response to Irofulven

A characteristic feature of cells from patients with Fanconi anemia is the formation of chromosome aberrations (breaks and radials) following treatment with DNA cross-linking agents $(1,2)$. We have observed that irofulven induces FANCD2 monoubiquitination and nuclear foci formation. These findings, together with our previous observations that irofulven activates ATM and its target genes NBS1, SMC1, and CHK2 (44), suggest that irofulven induces DNA double-strand breaks. Therefore, we hypothesize that FANCD2 might play a pivotal role in maintaining chromosome integrity, thereby affecting chemosensitivity in response to irofulven.

To assess the role that FANCD2 might play in maintaining chromosome integrity upon irofulven treatment, mitotic spread experiments were done in the vector and wild-type FANCD2-transfected PD20F fibroblasts. Chromosome aberrations (breaks and radials) were observed after irofulven treatment (Fig. 3A), indicating that irofulven does induce DNA double-strand breaks. Chromosome breaks and radials were observed more frequently in vector-transfected than in FANCD2-transfected PD20F cells after irofulven treatment (Fig. 3B and C), suggesting that FANCD2 plays a critical role in repairing DNA damage and maintaining chromosome integrity in response to irofulven-induced DNA damage.

To further confirm the role that FANCD2 plays in maintaining chromosome integrity in response to irofulven, the RNA interference approach was again used. Three shFANCD2 constructs (sh-F1, sh-F2, and sh-F3) and the control vector $(\mathrm{sh}-\mathrm{V})$ were stably transfected into SKOV3 cells. The effectiveness of these three shFANCD2 constructs in knocking-down the endogenous FANCD2 was verified by Western blotting. As shown in Fig. 4A, sh-F1 is the most effective construct in knocking-down FANCD2, therefore, the sh-F1 and sh-V-transfected SKOV3 cells were used in subsequent studies.

When mitotic cells of the vector (sh-V) and shFANCD2 (sh-F1)-transfected SKOV3 cells were analyzed after irofulven treatment, chromosome breaks and radials were observed in vector-transfected cells (Fig. 4B and C). However, in sh-F1-transfected cells, more severe chromosome damage was observed. In these metaphase cells, chromosomes were damaged to the point where all of the chromosomes seemed to be broken or fragmented (Fig. 4B and $\mathrm{C}$ ). These results further showed that FANCD2 is critical for maintaining chromosome integrity in response to irofulven-induced DNA damage.

\section{FANCD2 Confers Chemoresistance to Irofulven}

Based on the above observations that FANCD2 is activated and is important for chromosome integrity in response to irofulven-induced DNA damage, we hypothesize that FANCD2 might affect chemosensitivity to irofulven. To determine whether FANCD2 affects chemosensitivity to irofulven, the clonogenic survival assay was conducted. The vector, wild-type, or K561R mutant FANCD2-transfected PD20F fibroblasts were treated with different concentrations of irofulven for $1 \mathrm{~h}$ followed by drug-free incubations. When $\mathrm{IC}_{50}$ concentrations were compared, the results showed that FANCD2-transfected cells were 2 -fold more resistant to irofulven than vector or K561R mutant FANCD2-transfected cells (Fig. 5A). This finding was further supported by the clonogenic 
survival assay in vector and shFANCD2-transfected SKOV3 cells, where knocking-down the endogenous FANCD2 by RNA interference resulted in a $>4$-fold increase in chemosensitivity to irofulven when $\mathrm{IC}_{50}$ concentrations were compared (Fig. 5B). We also conducted a clonogenic assay with longer exposure times to verify that FANCD2-depleted cells were more sensitive. The results indicated that at $0.25 \mu \mathrm{mol} / \mathrm{L}$, a concentration that caused no difference in chemosensitivity when used for treatment for only $1 \mathrm{~h}$ (Fig. 5B), the shFANCD2transfected cells were $\sim 4$-fold or 24 -fold more sensitive than the vector-transfected cells when treated for 6 or 24 $\mathrm{h}$, respectively (Fig. 5C). Taken together, these results show that FANCD2 expression confers chemoresistance to irofulven, and FANCD2 monoubiquitination is the determining factor for FANCD2-mediated chemoresistance to irofulven.

\section{Discussion}

In this study, by using cell lines proficient or deficient for FANCD2, ATR, or ATM, we have shown that irofulven induces the monoubiquitination and foci formation of FANCD2. Irofulven-induced FANCD2 monoubiquitination is mediated by ATR. FANCD2 is critical for maintaining chromosome integrity and modulating chemosensitivity in response to irofulven.

There was some increase in the total amount of FANCD2 proteins observed in some cells following irofulven treatment (Fig. 1A). This could be the result of relatively overloading the protein extracts because it was not seen in other cells following irofulven treatment (Fig. 1A and C and Fig. 2B and C).

It has been reported that FANCD2 was phosphorylated by ATM or ATR in response to IR or psoralen/UV-A
$(52,53)$. The phosphorylation of FANCD2 is required for radiation-induced $\mathrm{S}$ phase cell cycle checkpoint, but not chemoresistance to mitomycin C (52). In this study, a slight shifting of both FANCD2-L and FANCD2-S bands following irofulven treatment was also observed (Figs. 1 and 2), indicating that FANCD2 was also phosphorylated in response to irofulven-induced DNA damage. This suggests that FANCD2 might also play some role in irofulveninduced cell cycle arrest.

The Fanconi anemia-BRCA pathway of DNA damage response is frequently impaired in cancers due to genetic mutation or epigenetic repression $(1,2,4,9,10,19)$. The findings of this study suggest that FANCD2 activation might be used as a potential predictive marker for chemosensitivity to irofulven therapy. Furthermore, the findings of this study also suggest that the genetic defects in other proteins of the Fanconi anemia-BRCA pathway may also be exploited for individualized therapy by irofulven. Further studies are warranted to determine whether these proteins will also influence the chemosensitivity to irofulven.

To date, the structure and nature of irofulven-induced DNA damage have not been characterized. Earlier studies suggested that the DNA damage caused by the illudin family of compounds might be repaired by the nucleotide excision repair pathway $(41,42)$. A recent study indicated that transcription-coupled nucleotide excision repair is the exclusive pathway in repairing illudin $\mathrm{S}$ and irofulvenelicited DNA lesions; base excision repair and nonhomologous end-joining of DNA double-strand break repair do not play a major role (43). However, in these studies, the homologous recombination pathway for double-strand break repair was not evaluated $(41-43)$. Recent reports indicated that ATM and CHK2 are specifically activated by drug or radiation-induced DNA double-strand breaks $(45,46)$. Our previous studies showed that irofulven activates ATM and
A

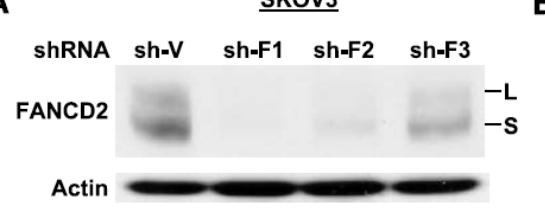

B

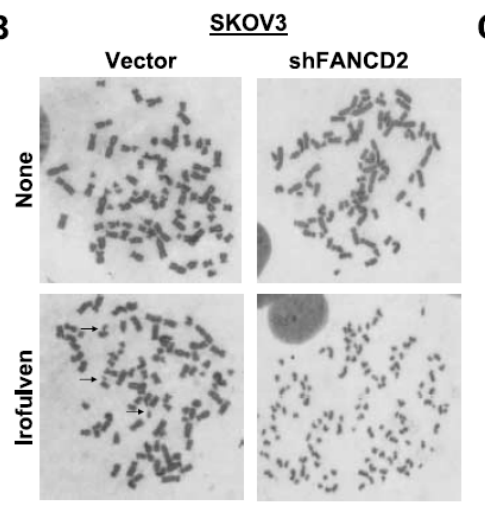

C

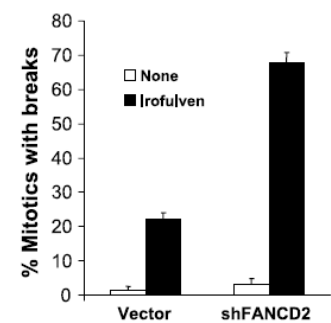

Figure 4. Knocking-down FANCD2 by RNA interference results in increased chromosome aberrations. A, SKOV3 cells were stably transfected with the vector $(s h-V)$ or shFANCD2 constructs (sh-F1, F2, and $F 3$ ), respectively. The efficacy of shFANCD2 constructs in knocking-down the endogenous FANCD2 was determined by Western blot analysis. B and C, the vector (sh-V) and shFANCD2 (sh-F1)-transfected SKOV3 cells were treated with 2.3 $\mu \mathrm{mol} / \mathrm{L}$ of irofulven for $1 \mathrm{~h}$ followed by $24 \mathrm{~h}$ of drug-free incubation. Mitotic spread staining was done. Arrows, chromosome breaks and radials (B). Columns, the mean percentage of mitotic cells with four or more chromosome breaks from triplicate counts of 50 cells; bars, SD (C). 


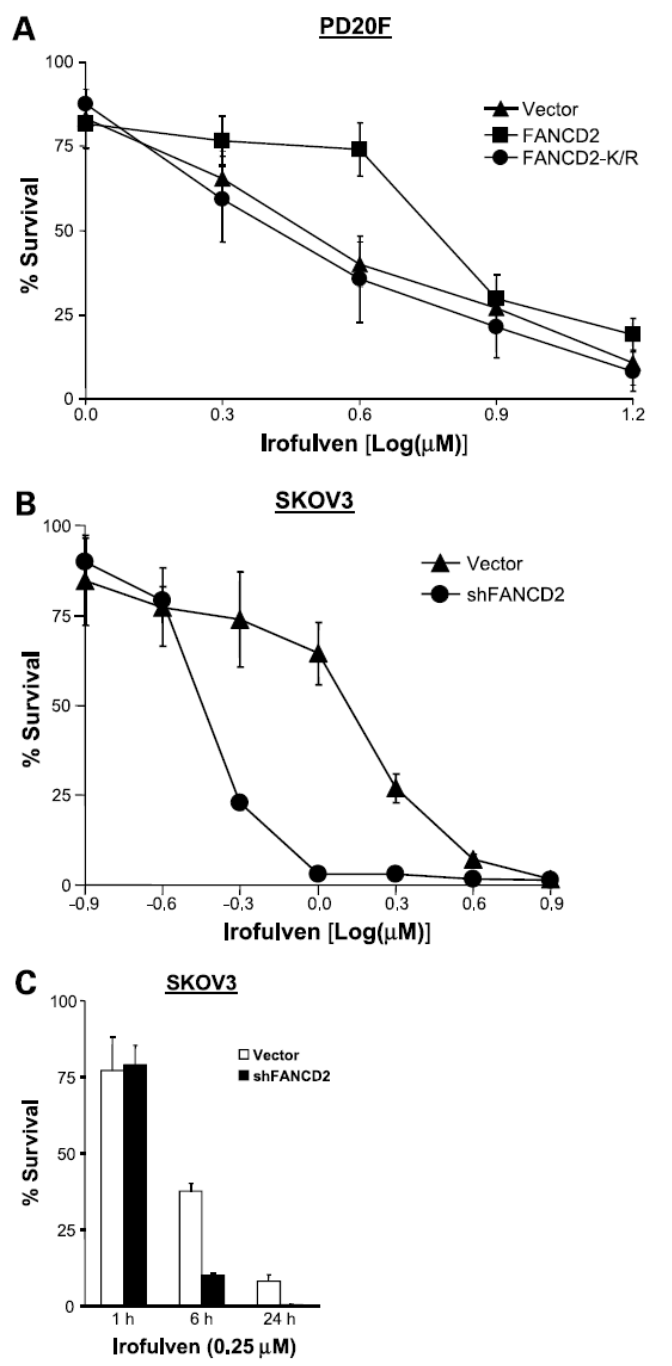

Figure 5. FANCD2 confers chemoresistance to irofulven. Chemo sensitivity was determined by clonogenic survival assay. Cells were treated with irofulven for $1 \mathrm{~h}$ followed by drug-free incubations. The mean and SD of triplicate experiments were presented. A, clonogenic survival of the vector, wild-type FANCD2, or K561R mutant FANCD2-transfected PD2OF fibroblasts treated with 1 to $16 \mu \mathrm{mol} / \mathrm{L}$ of irofulven for $1 \mathrm{~h}$. B D2OF clonogenic survival of the vector (sh-V) and shFANCD2 (sh-F1)-transfected SKOV 3 cells treated with 0.125 to $8 \mu \mathrm{mol} / \mathrm{L}$ of irofulven for $1 \mathrm{~h}$. C clonogenic survival of the sh-V and sh-F1-transfected SKOV 3 cells treate with $0.25 \mu \mathrm{mol} / \mathrm{L}$ of irofulven for 1,6 , or $24 \mathrm{~h}$.

its targets, NBS1, SMC1, CHK2, and p53 (44). These findings suggest that irofulven causes the generation of DNA doublestrand breaks. In support of this, we show here that irofulven induces chromosome aberrations (breaks and radials).
Several groups reported that monoubiquitinated FANCD2 colocalizes with BRCA1, BRCA2, and RAD51 in response to DNA damage, indicating that FANCD2 is required for homology-directed repair of double-strand breaks $(1,2,4,16,17,50,55)$. A recent study showed that FANCD2 binds to double-strand DNA ends and Holliday junctions (56). Other studies indicated that FANCD2 is important for maintaining chromosome fragile site stability (57) and may play a role in the cellular response to stalled DNA replication forks or in the repair of replicationassociated double-strand breaks $(17,57,58)$. The chromosome aberrations (breaks and radials) observed after irofulven treatment are also reminiscent of Fanconi anemia and BRCA2-deficient cells treated with the DNA crosslinking agent mitomycin $C(1,2,21,59)$. Therefore, it can be postulated that irofulven might cause stalled DNA replication, leading to the generation of DNA double-strand breaks. In this study, we found that irofulven-induced FANCD2 monoubiquitination is mediated by ATR, and that FANCD2 monoubiquitination directly contributes to chemoresistance to irofulven. Therefore, it is conceivable that ATR is also activated by irofulven and plays an important role in modulating the chemosensitivity to irofulven. How ATR controls irofulven-induced FANCD2 monoubiquitination is currently unknown. It could be that ATR activates the essential upstream protein components or ubiquitin ligases that are critical for FANCD2 monoubiquitination. Further studies are needed to elucidate the precise mechanisms that lead to the generation of double-strand breaks, and how other DNA damage signaling proteins might control the chemosensitivity in response to irofulven.

In summary, we have observed that irofulven induces FANCD2 monoubiquitination/activation and foci formation. Irofulven-induced FANCD2 monoubiquitination is mediated by ATR. FANCD2 is crucial for maintaining chromosome integrity and modulating chemosensitivity in response to irofulven-induced DNA damage. These findings will enhance our understanding of the mechanisms of action involved with irofulven, and more specifically, the proteins and mechanisms that might affect chemosensitivity to irofulven. These findings will also provide insight for additional studies of targeted therapy by irofulven in cancers defective for proteins of the Fanconi anemia-BRCA pathway.

\section{Acknowledgments}

We thank Dr. Alan D'Andrea (Dana-Farber Cancer Institute, Harvard Medical School, Boston, MA) for generously providing the vector, wild type, and K561R mutant FANCD2-infected Fanconi anemia D2 fibroblasts; Drs. S.L. Schreiber and S. Handeli for the GM00847 human fibroblast expressing kinase-dead ATR; and Dr. Y. Shiloh for the ATM-complemented AT fibroblast (AT22IJE-T-pEBS7-YZ5). We also thank Dr. Linda Sargent for the use of microscopes, and Emily Van Laar and Shannon Wadman for critical reading of the manuscript.

\section{References}

1. Venkitaraman AR. Tracing the network connecting BRCA and Fanconi anaemia proteins. Nat Rev Cancer 2004:4:266 - 76 .

2. D'Andrea AD, Grompe M. The Fanconi anaemia/BRCA pathway. Nat Rev Cancer 2003;3:23-34. 
3. Timmers C, Taniguchi T, Hejna J, et al. Positional cloning of a novel Fanconi anemia gene, FANCD2. Mol Cell 2001;7:241-8.

4. Garcia-Higuera I, Taniguchi T, Ganesan $S$, et al. Interaction of the Fanconi anemia proteins and BRCA1 in a common pathway. Mol Cell $2001 ; 7: 249-62$.

5. Meetei AR, Yan Z, Wang W. FANCL replaces BRCA1 as the likely ubiquitin ligase responsible for FANCD2 monoubiquitination. Cell Cycle $2004 ; 3: 179-81$

6. Thompson $\mathrm{LH}$. Unraveling the Fanconi anemia-DNA repair connection. Nat Genet 2005;37:921 - 2.

7. Mosedale G, Niedzwiedz W, Alpi A, et al. The vertebrate Hef ortholog is a component of the Fanconi anemia tumor-suppressor pathway. Nat :763- 71

8. Litman R, Peng M, Jin Z, et al. BACH1 is critical for homologous recombination and appears to be the Fanconi anemia gene product FANCJ. Cancer Cell 2005;8:255-65.

9. Medhurst AL, Huber PA, Waisfisz Q, de Winter JP, Mathew CG. Direct interactions of the five known Fanconi anaemia proteins suggest common functional pathway. Hum Mol Genet 2001;10:423-9.

10. Meetei AR, Sechi S, Wallisch M, et al. A multiprotein nuclear complex connects Fanconi anemia and Bloom syndrome. Mol Cell Biol 2003;23 $3417-26$.

11. Meetei AR, de Winter JP, Medhurst AL, et al. A novel ubiquitin ligase is deficient in Fanconi anemia. Nat Genet 2003;35:165-70.

12. Meetei AR, Levitus $M, X u e ~ Y$, et al. X-linked inheritance of Fancon anemia complementation group B. Nat Genet 2004;36:1219-24.

13. Meetei AR, Medhurst AL, Ling $C$, et al. A human ortholog of archaea DNA repair protein Hef is defective in Fanconi anemia complementation group M. Nat Genet 2005;37:958 - 63 .

14. Nijman SM, Huang TT, Dirac AM, et al. The deubiquitinating enzyme USP1 regulates the Fanconi anemia pathway. Mol Cell 2005; 17:331-9. 15. Howlett NG, Taniguchi T, Olson S, et al. Biallelic inactivation of BRCA2 in Fanconi anemia. Science 2002;297:606 - 9.

16. Wang $X$, Andreassen $P R$, $D^{\prime}$ Andrea $A D$. Functional interaction of monoubiquitinated FANCD2 and BRCA2/FANCD1 in chromatin. Mol Cel Biol 2004;24:5850-62

17. Hussain S, Wilson JB, Medhurst AL, et al. Direct interaction of FANCD2 with BRCA2 in DNA damage response pathways. Hum Mo Genet 2004; $13: 1241-8$.

18. Vandenberg CJ, Gergely F, Ong CY, et al. BRCA1-independent ubiquitination of FANCD2. Mol Cell 2003:12:247-54.

19. Turner N, Tutt A, Ashworth A. Hallmarks of 'BRCAness' in sporadic cancers. Nat Rev Cancer 2004;4:814-9.

20. Narod SA, Foulkes WD. BRCA1 and BRCA2: 1994 and beyond. Nat Rev Cancer 2004;4:665 - 76.

21. Venkitaraman AR. Cancer susceptibility and the functions of BRCA 1 and BRCA2. Cell 2002;108:171-82.

22. van der Heijden MS, Yeo CJ, Hruban RH, Kern SE. Fanconi anemia gene mutations in young-onset pancreatic cancer. Cancer Res 2003;63: $2585-8$.

23. Rogers CD, Van Der Heijden MS, Brune K, et al. The Genetics of FANCC and FANCG in Familial Pancreatic Cancer. Cancer Biol Ther 2004 3:167-9.

24. Martin ST, Matsubayashi H, Rogers CD, et al. Increased prevalence of the BRCA2 polymorphic stop codon $\mathrm{K} 3326 \mathrm{X}$ among individuals with familial pancreatic cancer. Oncogene 2005;24:3652-6.

25. Rogers CD, Couch FJ, Brune K, et al. Genetics of the FANCA gene in familial pancreatic cancer. J Med Genet 2004;41:e126.

26. Goggins M, Hruban RH, Kern SE. BRCA2 is inactivated late in the development of pancreatic intraepithelial neoplasia: evidence and implications. Am J Pathol 2000; 156:1767- 71.

27. Arnold MA, Goggins M. BRCA2 and predisposition to pancreatic and other cancers. Expert Rev Mol Med 2001;2001:1 - 10.

28. Hughes-Davies L, Huntsman D, Ruas M, et al. EMSY links the BRCA2 pathway to sporadic breast and ovarian cancer. Cell 2003;1 15:523-35.

29. Narayan G, Arias-Pulido H, Nandula SV, et al. Promoter hypermethylation of FANCF: disruption of Fanconi anemia-BRCA pathway in cervical cancer. Cancer Res 2004;64:2994-7.

30. Marsit CJ, Liu M, Nelson HH, Posner M, Suzuki M, Kelsey KT. Inactivation of the Fanconi anemia/BRCA pathway in lung and ora cancers: implications for treatment and survival. Oncogene 2004;23: $1000-4$

31. Taniguchi $T$, Tischkowitz $M$, Ameziane $N$, et al. Disruption of the Fanconi anemia-BRCA pathway in cisplatin-sensitive ovarian tumors. Nat Med 2003;9:568- 74

32. MacDonald JR, Muscoplat CC, Dexter DL, et al. Preclinical antitumo activity of 6-hydroxymethylacylfulvene, a semisynthetic derivative of the mushroom toxin illudin S. Cancer Res 1997;57:279-83.

33. Sato $Y$, Kashimoto $S$, MacDonald JR, Nakano K. In vivo antitumou efficacy of MGI-114 (6-hydroxymethylacylfulvene, HMAF) in various human tumour xenograft models including several lung and gastric tumours. Eur J Cancer 2001:37:1419-28.

34. Friedman HS, Keir ST, Houghton PJ, Lawless AA, Bigner DD, Waters SJ. Activity of irofulven (6-hydroxymethylacylfulvene) in the treatment of glioblastoma multiforme-derived xenografts in athymic mice. Cancer Chemother Pharmacol 2001;48:413-6.

35. Hidalgo M, Izbicka E, Eckhardt SG, et al. Antitumor activity of MG 114 (6-hydroxymethylacylfulvene, HMAF), a semisynthetic derivative of illudin S, against adult and pediatric human tumor colony-forming units. Anticancer Drugs 1999;10:837-44.

36. Britten CD, Hilsenbeck SG, Eckhardt SG, et al. Enhanced antitumor activity of 6-hydroxymethylacylfulvene in combination with irinotecan and 5 -fluorouracil in the HT 29 human colon tumor xenograft model. Cancer Res 1999;59:1049- 53.

37. Murgo A, Cannon DJ, Blatner G, Cheson BD. Clinical trials referra resource. Clinical trials of MGI-114. Oncology (Williston Park) 1999:13. 233, 237-8.

38. Kelner MJ, McMorris TC, Rojas RJ, et al. Enhanced antitumor activity of irofulven in combination with antimitotic agents. Invest New Drugs $2002 ; 20: 271-9$.

39. Senzer N, Arsenau J, Richards D, Berman B, MacDonald JR, Smith S. Irofulven demonstrates clinical activity against metastatic hormone refractory prostate cancer in a phase 2 single-agent trial. Am J Clin Oncol refractory prostate $28: 36-42$.

40. Woo MH, Peterson JK, Billups C, Liang H, Bjornsti MA, Houghton PJ. Enhanced antitumor activity of irofulven in combination with irinotecan in pediatric solid tumor xenograft models. Cancer Chemother Pharmaco $2005 ; 55: 411-9$.

41. Kelner MJ, McMorris TC, Estes $L$, et al. Characterization of illudin $S$ sensitivity in DNA repair-deficient Chinese hamster cells. Unusually high sensitivity in DNA repairdeficient Chine hamster cells. Unusually high compris to comparison to other chemotherapeutic agents. Biochem Pharmaco
1994:48:403-9. 42. Kelner MJ, McMorris TC, Estes L, et al. Efficacy of Acylfulvene Illudin analogues against a metastatic lung carcinoma MV522 xenograft nonresponsive to traditional anticancer agents: retention of activity against various mdr phenotypes and unusual cytotoxicity against ERCC2 and ERCC3 DNA helicase-deficient cells. Cancer Res 1995; $55: 4936-40$.

43. Jaspers NG, Raams A, Kelner MJ, et al. Anti-tumour compounds illudin $\mathrm{S}$ and Irofulven induce DNA lesions ignored by global repair and exclusively processed by transcription- and replication-coupled repair pathways. DNA Repair (Amst) 2002:1:1027-38.

44. Wang J, Wiltshire $T$, Wang $Y$, et al. ATM-dependent CHK2 activation induced by anticancer agent, irofulven. J Biol Chem 2004, 279:39584-92

45. Ismail IH, Nystrom S, Nygren J, Hammarsten O. Activation of ataxia telangiectasia mutated by DNA strand break-inducing agents correlates
closely with the number of DNA double strand breaks. J Biol Chem 2005; 280:4649-55.

46. Buscemi G, Perego P, Carenini N, et al. Activation of ATM and Chk2 kinases in relation to the amount of DNA strand breaks. Oncogene 2004, 23: $7691-700$

47. Ziv $Y$, Bar-Shira A, Pecker I, et al. Recombinant ATM protein complements the cellular A-T phenotype. Oncogene 1997; 15:159-67.

48. Cliby WA, Roberts CJ, Cimprich KA, et al. Overexpression of a kinaseinactive ATR protein causes sensitivity to DNA-damaging agents and defects in cell cycle checkpoints. EMBO J 1998;17:159-69.

49. Casper AM, Nghiem P, Arlt MF, Glover TW. ATR regulates fragile site stability. Cell 2002;111:779-89.

50. Taniguchi T, Garcia-Higuera I, Andreassen PR, Gregory RC Grompe M, D'Andrea AD. S-phase-specific interaction of the Fancon 
anemia protein, FANCD2, with BRCA1 and RAD51, Blood 2002;100: 2414-20.

51. Husain A, He G, Venkatraman ES, Spriggs DR. BRCA1 up-regulation is associated with repair-mediated resistance to cis-diamminedichloroplatinum(II). Cancer Res 1998;58:1120-3.

52. Taniguchi T, Garcia-Higuera I, Xu B, et al. Convergence of the Fanconi anemia and ataxia telangiectasia signaling pathways. Cell 2002:109: $459-72$.

53. Pichierri P, Rosselli F. The DNA crosslink-induced S-phase checkpoint depends on ATR-CHK1 and ATR-NBS1-2 pathways. EMBO J 2004;23: $1178-87$

54. Andreassen PR, D'Andrea AD, Taniguchi T. ATR couples FANCD2 monoubiquitination to the DNA-damage response. Genes Dev 2004;18: $1958-63$

55. Nakanishi K, Yang YG, Pierce AJ, et al. Human Fanconi anemia monoubiquitination pathway promotes homologous DNA repair. Proc Nat Acad Sci U S A 2005:102:1110-5.

56. Park WH, Margossian S, Horwitz AA, Simons AM, D'Andrea AD, Parvin JD. Direct DNA binding activity of the Fanconi anemia D2 protein. Biol Chem 2005;280:23593-8.

57. Howlett NG, Taniguchi T, Durkin SG, D'Andrea AD, Glover TW. The Fanconi anemia pathway is required for the DNA replication stress response and for the regulation of common fragile site stability. Hum Mol Genet 2005;14:693-701.

58. Rothfuss A, Grompe M. Repair kinetics of genomic interstrand DNA cross-links: evidence for DNA double-strand break-dependent activation of the Fanconi anemia/BRCA pathway. Mol Cell Biol 2004; $24: 123-34$

59. Shiloh Y. ATM and related protein kinases: safeguarding genome integrity. Nat Rev Cancer 2003;3:155-68. 


\section{Appendix 2}

Irofulven induces replication-dependent CHK2 activation related to $\mathrm{p} 53$ status

This manuscript was published in Biochemical Pharmacology 


\title{
Irofulven induces replication-dependent CHK2 activation related to p53 status
}

\author{
Yutian Wang ${ }^{a}$, Timothy Wiltshire ${ }^{b}$, Jamie Senft $^{a}$, Eddie Reed $^{a, b, 1}$, Weixin Wang ${ }^{a, b, *}$ \\ ${ }^{a}$ Mary Babb Randolph Cancer Center, West Virginia University School of Medicine, Morgantown, WV 26506, United States \\ ${ }^{\mathrm{b}}$ Department of Microbiology, Immunology and Cell Biology, West Virginia University School of Medicine, Morgantown, WV 26506, \\ United States
}

\section{A R T I C L E I N F O}

Article history:

Received 2 August 2006

Accepted 18 October 2006

\section{Keywords:}

CHK2

p53

Replication

Chemosensitivity

Cell cycle

Irofulven

\begin{abstract}
A B S T R A C T
CHK2 and p53 are frequently mutated in human cancers. CHK2 is known to phosphorylate and stabilize p53. CHK2 has also been implicated in DNA repair and apoptosis induction. However, whether p53 affects CHK2 activation and whether CHK2 activation modulates chemosensitivity are unclear. In this study, we found that in response to the DNA damage agent, irofulven, CHK2 activation, rather than its expression, is inversely correlated to p53 status. Irofulven inhibits DNA replication and induces chromosome aberrations (breaks and radials) and p53-dependent cell cycle arrest. Pretreatment of cells with the DNA polymerase inhibitor, aphidicolin, resulted in reduction of irofulven-induced CHK2 activation and foci formation, indicating that $\mathrm{CHK} 2$ activation by irofulven is replication-dependent. Furthermore, by using ovarian cancer cell lines expressing dominant-negative CHK2 and CHK2knockout HCT116 cells, we found that CHK2 activation contributes to the control of S and G2/M cell cycle arrests, but not chemosensitivity to irofulven. Overall, this study demonstrates that in response to irofulven-induced DNA damage, the activation of CHK2 is dependent on DNA replication and related to p53 status. By controlling cell cycle arrest and DNA replication, p53 affects CHK2 activation. CHK2 activation contributes to cell cycle arrest, but not chemosensitivity.
\end{abstract}

(C) 2006 Elsevier Inc. All rights reserved.
1.

Introduction

In response to DNA damage, cells evoke signal transduction pathways to arrest at G1/S, S or G2/M phases, allowing time to deal with the insult [1,2]. DNA damage activates ATM (ataxia telangiectasia-mutated) and/or ATR (ATM-Rad3-related) kinases, which in turn phosphorylate and activate downstream effector kinases, CHK1 and CHK2. ATM phosphorylates CHK2 on Thr68 leading to CHK2 kinase activation, while ATM and ATR phosphorylate CHK1 on Ser317 and Ser345 resulting in its activation. Activation of CHK1 and CHK2 regulates $\mathrm{S}$ phase by phosphorylating $\mathrm{CDC} 25 \mathrm{~A}$, or the $\mathrm{G} 2 / \mathrm{M}$ transition by phosphorylating CDC25C [2-4]. ATM and ATR phosphorylate p53 on Ser15; and CHK1 and CHK2 phosphorylate p53 on Ser20, leading to its accumulation and activation [2-4]. Activation of p53 initiates cell cycle arrest and DNA repair-related genes such as p21, GADD45 and 14-3-38, which leads to G1 and G2 arrest.

Tumor suppressors $\mathrm{p} 53$ and CHK2 are frequently mutated in human cancers $[5,6]$. Besides their roles in cell cycle control,

\footnotetext{
* This work was supported by a grant from the National Cancer Institute (R03CA107979 to W.W.).

* Corresponding author at: Mary Babb Randolph Cancer Center, West Virginia University School of Medicine, Morgantown, WV 26506, United States. Tel.: +1 304293 2243; fax: +1 3042934667 .

E-mail address: wwang@hsc.wvu.edu (W. Wang).

${ }^{1}$ Present address: Center for Disease Control and Prevention, Atlanta, Georgia, United States. 0006-2952/\$ - see front matter (C) 2006 Elsevier Inc. All rights reserved. doi:10.1016/j.bcp.2006.10.023
} 
p53 and CHK2 are also involved in regulating DNA repair and apoptosis [5-7]. p53 promotes DNA repair or apoptosis by directly regulating the expression of genes such as p48, DR5, BAX, PUMA and NOXA [2,8]. CHK2 (cds1) in fission yeast was shown to interact with RAD60, a protein required for recombinational repair, at stalled replication forks [9]. In mammalian cells, it was shown that CHK2 promotes homologous recombination (HR) and non-homologous end-joining (NHEJ) of DNA double-strand breaks through phosphorylation of BRCA1 on serine 988 [10-13].

CHK2 has also been implicated in apoptosis induction. However, there are conflicting studies. Some studies found that $\mathrm{CHK} 2$ is a negative regulator of mitotic catastrophe, and inhibition of CHK2 sensitized cells to chemotherapy-induced apoptosis $[14,15]$. Other studies found that CHK2 is involved in apoptosis induction [16-23]. Additional studies indicated that CHK2 induces apoptosis by activating E2F1, which in turn upregulates its target genes INK4a/ARF, Apaf-1, caspase-7, p73 and p53 [24-26].

While many studies have investigated how CHK2 activates and stabilizes p53 $[6,17,27-29]$, the possible role of p53 in regulating CHK2 protein level or activity has not been well studied. An inverse correlation between p53 and CHK2 expression has been reported in tumor cell lines and tissue specimens [30,31]. A recent report suggested that p53 negatively regulates $\mathrm{CHK} 2$ expression by modulating the function of transcription factor NF-Y [32].

Given the critical roles that CHK2 plays in cell cycle control, DNA repair and apoptosis, it is still unclear whether it can ultimately affect chemosensitivity. In this study, we investigated the possible role that p53 plays in CHK2 activation and whether CHK2 activation will affect chemosensitivity in response to the DNA damage agent, irofulven. Irofulven (6-hydroxymethylacylfulvene, NSC\# 683863) is a semi-synthetic analog of mushroom-derived illudin toxins. Preclinical studies and clinical trials have demonstrated that it is effective against several tumor cell types [33-41]. It was previously demonstrated that irofulven activates ATM and its targets, NBS1, SMC1, CHK2 and p53 [42]. Here, we found that CHK2 activation is DNA replication-dependent and related to p53 status. By controlling cell cycle arrest and DNA replication, p53 affects $\mathrm{CHK} 2$ activation. Furthermore, we demonstrated that $\mathrm{CHK} 2$ activation contributes to the control of $\mathrm{S}$ and $\mathrm{G} 2 / \mathrm{M}$ cell cycle arrest, but not chemosensitivity, in response to irofulven-induced DNA damage.

\section{2. \\ Materials and methods}

\subsection{Cell culture}

All cell lines were maintained in various media supplemented with $10 \%$ fetal bovine serum in a $37{ }^{\circ} \mathrm{C}$ incubator with $5 \% \mathrm{CO}_{2}$ atmosphere. Ovarian cancer cell lines A2780 and CAOV3 were cultured in RPMI 1640. Colon cancer cell line HCT116 and its p53-knockout (HCT116 p53-/-) [43] and CHK2-knockout (HCT116 CHK2-/-) cells [44] (generously provided by Prof. Bert Vogelstein, Johns Hopkins University, Baltimore, MD) were cultured in McCoy's 5A medium.

\subsection{Clonogenic survival assay}

To determine chemosensitivity and $1 \times$ IC50 concentration, the clonogenic survival assay was performed on $60-\mathrm{mm}$ cell culture dishes as described previously [42]. Cells were treated with different concentrations of irofulven for $1 \mathrm{~h}$ followed by drugfree incubations for about 10 days. Colonies were stained with crystal violet and counted if 50 or more cells were present.

\subsection{Western blotting}

Western blot was performed as described previously [42]. The antibodies used included: monoclonal anti-actin (Sigma, St. Louis, MO), polyclonal anti-phosphorylated CHK2 on Thr 68 and anti-CHK2 (Cell Signaling Technology, Beverly, MA), monoclonal anti-p53, polyclonal anti-p21, anti-cyclin E, A, and B1 (Santa Cruz Biotechnology, Santa Cruz, CA), and monoclonal anti-HA (Roche Applied Science, Indianapolis, IN).

\subsection{Propidium iodide staining and flow cytometry}

Cells were harvested, washed once with cold PBS and fixed in $75 \%$ ethanol/PBS. After washing twice with PBS, cells were resuspended in PBS containing $10 \mu \mathrm{g} / \mathrm{ml}$ propidium iodide, $20 \mu \mathrm{g} /$ $\mathrm{ml}$ RNase A, $0.1 \%$ sodium citrate and $0.1 \%$ Triton X-100. Cells were analyzed by FACSCalibur with CellquestPro software (Becton Dickinson, San Jose, CA). Cell cycle distributions were analyzed by ModFit v3.0 software (Verity, Topsham, ME).

\subsection{Metaphase spread}

Cells were treated with irofulven for $1 \mathrm{~h}$ followed by $24 \mathrm{~h}$ of drug-free incubation. Colcemid $(400 \mathrm{ng} / \mathrm{ml})$ (Biosciences, La Jolla, CA) was added to medium $4 \mathrm{~h}$ before harvesting. After trypsinization, cells were washed with PBS. Cell pellets were re-suspended in $75 \mathrm{mM} \mathrm{KCl}$ and placed in a $37^{\circ} \mathrm{C}$ incubator for $8 \mathrm{~min}$. After centrifugation, cells were fixed using 3:1 absolute methanol to glacial acetic acid at $4{ }^{\circ} \mathrm{C}$ for $2 \mathrm{~h}$ and then washed twice with fixative. Cells were re-suspended in fixative and dropped onto slides. Slides were air-dried at room temperature and stained with 5\% Gurr's Giemsa stain (Biomedical Specialties, Santa Monica, CA) for $7 \mathrm{~min}$. Slides were rinsed twice with distilled water and air-dried. The images were recorded with a Leica DMR microscope (Bannockburn, IL) and Applied Imaging digital system (San Jose, CA).

\subsection{BrdU incorporation and flow cytometry}

BrdU $(10 \mu \mathrm{M})$ (Roche, Indianapolis, IN) was added to cell culture $20 \mathrm{~min}$ before adding irofulven. Cells were harvested at different time points, washed once with cold PBS, and fixed in $75 \%$ ethanol/PBS. Staining for BrdU and flow cytometry were performed as described previously [42].

\subsection{Immunofluorescent staining}

Cells were plated on cover-slips and treated with irofulven for $1 \mathrm{~h}$ followed by $12 \mathrm{~h}$ of drug-free incubation. Cells were fixed and stained with polyclonal antibody against phosphorylated CHK2 on Thr 68 (Cell Signaling Technology, Beverly, MA). After 
staining with Alexafluor 488-conjugated goat anti-rabbit secondary antibody (Molecular Probes, Eugene, OR), slides were mounted with Vectashield mounting medium (Vector Laboratories, Burlingame, CA) containing $5 \mathrm{ng} / \mathrm{ml}$ of DAPI. Staining images were captured by an Olympus Provis AX70 fluorescence microscope (Olympus, Melville, NY), and Spot digital camera and software (Diagnostic Instruments, Sterling Height, MI).

\subsection{Stable transfection of dominant-negative CHK2}

The HA-tagged dominant-negative CHK2 (CHK2 kinase-dead, CHK2.kd) CDNA was PCR-amplified from the pEF-BOSCHK2.kd vector [45] (generously provided by Dr. Jann N. Sarkaria, Mayo Clinic and Foundation, Rochester, MN) and sub-cloned into the pCIneo vector (Promega, Madison, WI). The resulting plasmid was verified by DNA sequencing. The vector and HA-tagged CHK2.kd plasmids were subsequently transfected into the ovarian cancer cell line CAOV3. Stable cell lines harboring the vector and CHK2.kd were established by selecting in media containing G418 (Invitrogen, Carlsbad, CA).

\subsection{Immunoprecipitation and in vitro kinase assay}

The in vitro kinase assay was performed as described previously [46]. Immunoprecipitation was performed with polyclonal antibody against CHK2 (Santa Cruz Biotechnology, Santa Cruz, $\mathrm{CA}$ ). The GST-CDC25 $\mathrm{C}_{200-256}$ (generously provided by Dr. Jann N. Sarkaria, Mayo Clinic and Foundation, Rochester, MN) fusion proteins were expressed in bacteria and purified by affinity chromatography as described [45]. The purified GST$\mathrm{CDC}_{25} \mathrm{C}_{200-256}$ fusion proteins were used as the substrate.

\subsection{RDS assay}

The radioresistant DNA synthesis (RDS) assay was performed as described previously [47]. Briefly, cells in the logarithmic phase of growth were pre-labeled by culturing in medium containing $10 \mathrm{nCi}$ of $\left[{ }^{14} \mathrm{C}\right]$-thymidine (Perkin-Elmer, Boston, MA) for $24 \mathrm{~h}$. The medium was then replaced with normal medium, and cells were incubated for another $24 \mathrm{~h}$. Cells were treated with irofulven for $1 \mathrm{~h}$ and incubated in drug-free medium for $3 \mathrm{~h}$. Cells were then pulse-labeled with $2.5 \mu \mathrm{Ci}$ of $\left[{ }^{3} \mathrm{H}\right]$-thymidine (Perkin-Elmer, Boston, MA) for $15 \mathrm{~min}$. Cells were harvested, washed twice with PBS, and fixed in $70 \%$ methanol for $30 \mathrm{~min}$. Cells were then transferred to Whatman filters (Whatman, Clifton, NJ) and washed sequentially with $70 \%$ and then $95 \%$ methanol. The filters were air-dried and the amount of radioactivity was quantified in a Wallac 1410 liquid scintillation counter (Perkin-Elmer, Downers Grove, IL). The resulting ratio of ${ }^{3} \mathrm{H}$ to ${ }^{14} \mathrm{C}$ counts per minute, corrected for channel crossover, was a measure of DNA synthesis.

\subsection{Phosphorylated histone $\mathrm{H} 3$ staining and flow} cytometry

Phospho-histone H3 staining was done as described previously [47]. Briefly, cells were treated with irofulven for $1 \mathrm{~h}$ followed by 1 or $3 \mathrm{~h}$ of drug-free incubation. Cells were harvested and fixed in 70\% ethanol. The fixed cells were washed twice with PBS and made permeable with $0.25 \%$ Triton $\mathrm{X}-100$ in PBS on ice for $10 \mathrm{~min}$. Cells were rinsed in $1 \%$ BSA/PBS and then stained with rabbit anti-phospho-S10 histone H3 antibody (Upstate, Charlottesville, VA) for $2 \mathrm{~h}$ at room temperature. Cells were rinsed in $1 \% \mathrm{BSA} / \mathrm{PBS}$ and incubated with Alexafluor 488-conjugated anti-rabbit secondary antibody for $30 \mathrm{~min}$ at room temperature. Cells were washed twice with PBS and suspended in PBS containing propidium iodide $(0.25 \mu \mathrm{g} / \mathrm{ml})$ and RNase A $(20 \mu \mathrm{g} / \mathrm{ml})$. Flow cytometry was performed on FACSCalibur with CellquestPro software (Becton Dickinson, San Jose, CA). Thirty thousand events were recorded for each sample. The percentage of mitotic cells was determined as those cells that were Alexafluor-positive and contained $4 \mathrm{~N}$ DNA content.

\section{Results \\ 3.1. CHK2 activation induced by irofulven is related to $p 53$} status.

CHK2 is phosphorylated on Thr 68 in response to DNA damage, followed by oligomerization and auto-phosphorylation resulting in its activation [48-53]. CHK2 is known to phosphorylate and activate p53 $[6,17,27-29]$. Whether p53 will affect CHK2 activation in response to DNA damage is unclear. To investigate the roles that p53 might play in CHK2 activation, we determined CHK2 kinase activation by assessing Thr 68 phosphorylation using Western blot in p53-proficient and deficient cells after irofulven treatment. We found that CHK2

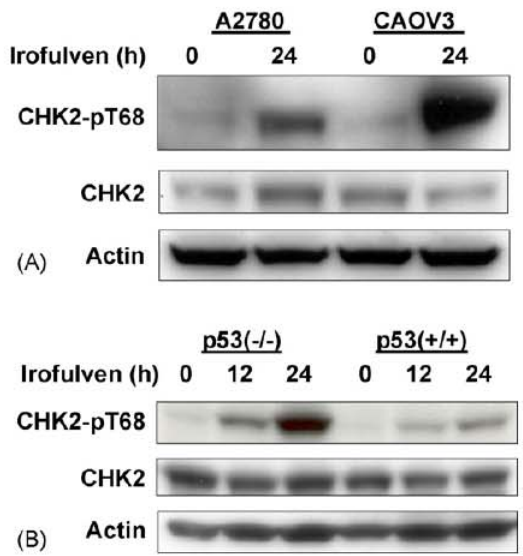

Fig. 1 - CHK2 activation induced by irofulven is related to p53 status. Ovarian cancer cell lines A2780 (p53 wild-type) and CAOV3 (p53-mutated) (A) and colon cancer cell line HCT116 and its p53-knockout subline (p53-l-) (B) were treated with $1 \times$ IC50 concentrations of irofulven $(0.8,0.9$ and $2.8 \mu \mathrm{M}$ for A2780, CAOV3 and HCT116 cells, respectively) for $1 \mathrm{~h}$ followed by 12 or $24 \mathrm{~h}$ of drug-free incubations. CHK2 activation was determined by Westem blot analysis with antibody recognizing $\mathrm{Thr} 68-$ phosphorylated CHK2 (CHK2-pT68). Blots for CHK2 and actin served as the loading control. 
activation, rather than its expression, is inversely correlated to p53 status. Stronger CHK2 activation was observed in p53mutated ovarian cancer cells (CAOV3) and p53-knockout colon cancer cells (HCT116 p53-/-) than in p53 wild-type ovarian cancer cells (A2780) and parental HCT116 (p53+/+) cells after irofulven treatment (Fig. 1).

\subsection{Irofulven induces p53-dependent cell cycle arrest}

Since CHK2 activation is related to $\mathrm{p} 53$ status in response to irofulven-induced DNA damage, we hypothesized that CHK2 activation might be related to p53-dependent cell cycle arrest and DNA replication. We analyzed the cell cycle
$\mathbf{A 2 7 8 0}$

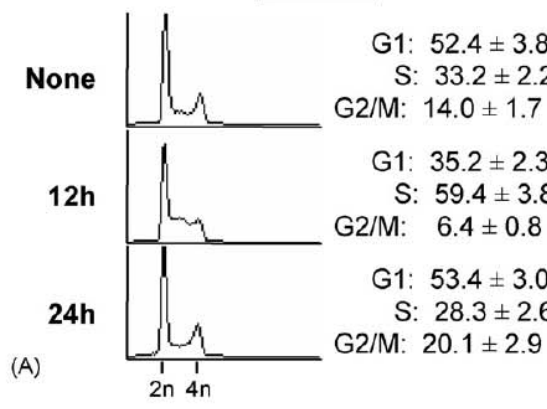

(B)

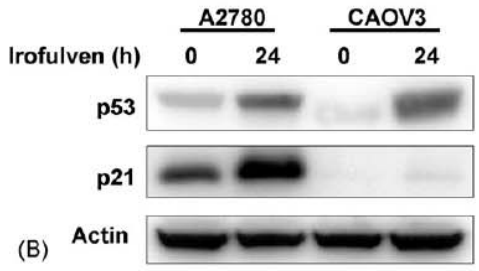

p53+/t

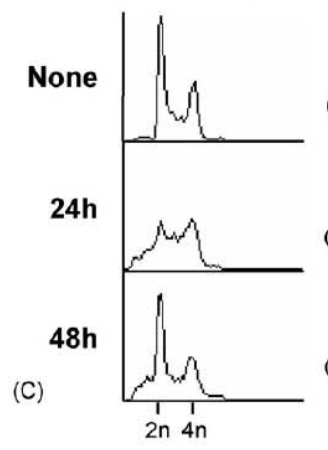

G1: $43.4 \pm 13.2$

S: $39.0 \pm 4.6$

G2/M: $17.6 \pm 8.6$

G1: $11.6 \pm 5.7$

S: $61.2 \pm 5.8$

G2/M: $27.2 \pm 1.2$

G1: $45.6 \pm 0.6$

S: $27.8 \pm 0.8$

G2/M: $26.6 \pm 0.6$

p53(-1-)

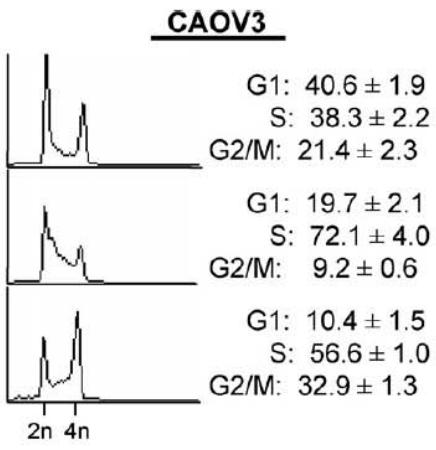

G1: $40.6 \pm 1.9$

G1: $19.7 \pm 2.1$

S: $72.1 \pm 4.0$
M: $9.2 \pm 0.6$

G1: $10.4 \pm 1.5$

S: $56.6 \pm 1.0$

$2 n 4 n$

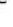


profiles in ovarian cancer cells A2780 (p53 wild-type) and CAOV3 (p53-mutated) using PI staining and FACS analysis. As shown in Fig. 2A, $12 \mathrm{~h}$ after drug treatment, A2780 cells displayed loss of G1 and increase of S phase. By $24 \mathrm{~h}$, the G1 peak was restored and there was a slight increase in $\mathrm{G} 2 / \mathrm{M}$ population. In CAOV3 cells, $12 \mathrm{~h}$ after drug treatment, loss of G1 and an increase of S phase were observed. By $24 \mathrm{~h}$, $S$ phase arrest and $\mathrm{G} 2 / \mathrm{M}$ accumulation were observed (Fig. 2A).

Consistent with the p53-dependent cell cycle arrest observed by FACS analysis, p53 accumulation and p21 induction after irofulven treatment were observed in p53 wild-type A2780 cells. In p53-mutated CAOV3 cells, the accumulation of $\mathrm{p} 53$ protein was observed, but p21 induc- tion was largely impaired (Fig. 2B). These data together indicated that irofulven induces p53-dependent cell cycle arrest.

To further confirm that irofulven induces p53-dependent cell cycle arrest, the cell cycle profiles of HCT116 p53+/+ and HCT 116 p53-/- cells were analyzed. These cells grew relatively slower than $\mathrm{A} 2780$ and CAOV3 cells, therefore, we examined the cell cycle profiles at 24 and $48 \mathrm{~h}$ after drug treatment. As shown in Fig. 2C, $24 \mathrm{~h}$ after drug exposure, both HCT116 p53+/+ and HCT116 p53-/- cells displayed some S phase arrest; while HCT116 p53-/- cells showed complete loss of $\mathrm{G} 1$ and more accumulation of $\mathrm{G} 2 / \mathrm{M}$ compared with parental HCT116 cells. Forty-eight hours after drug treatment, parental HCT116 cells were mainly
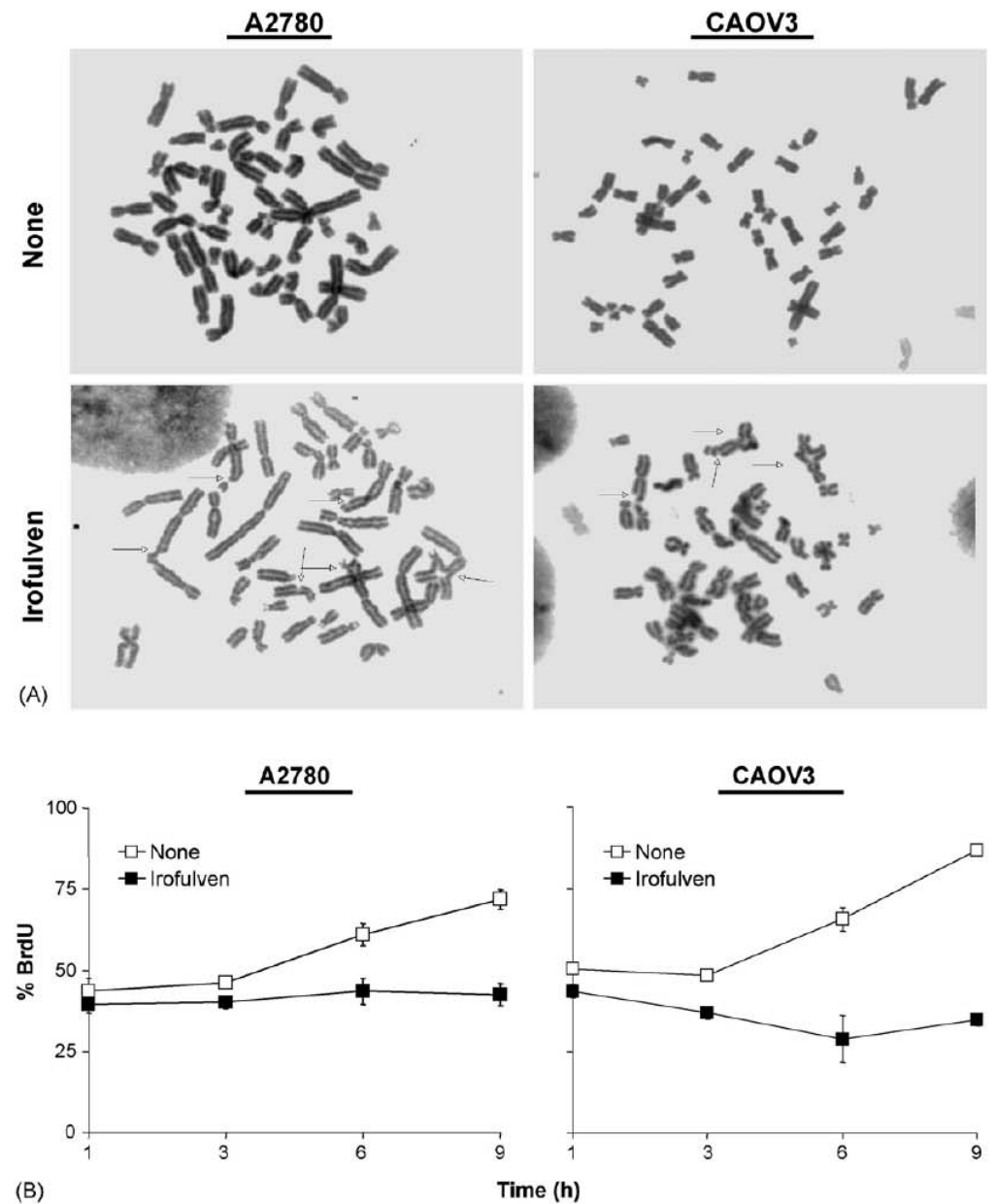

(B)

Time (h)

Fig. 3 - Irofulven induces chromosome aberrations and inhibits DNA synthesis. (A) A2780 and CAOV3 cells were treated with $1 \times$ IC50 concentrations of irofulven $(0.8$ and $0.9 \mu \mathrm{M}$, respectively) for $1 \mathrm{~h}$ followed by $24 \mathrm{~h}$ of drug-free incubations. Metaphase spread staining was performed. Arrows indicate chromosome breaks, triradials and quadriradials. (B) A2780 and CAOV3 cells were treated with $1 \times$ IC50 concentrations of irofulven for 1-9 h. DNA synthesis rate was assessed by BrdU incorporation and FACS analysis. The mean and standard deviation of triplicate experiments were shown. 
arrested at G1 phase, while large populations of HCT116 p53-/ - cells were arrested at G2/M phases (Fig. 2C). Corresponding to p53-dependent cell cycle arrest, p53 accumulation and p21 induction were observed in parental HCT116 cells, but not in p53-knockout cells after irofulven treatment (Fig. 2D). Taken together, these results demonstrated that irofulven induces p53-dependent cell cycle arrest; p53 wild-type cells mainly arrested at G1/S phases, while p53-mutated or p53-null cells arrested at S and G2/M phases.
3.3. Irofulven induces chromosome aberrations and inhibits DNA synthesis

To further explore the mechanisms that cause p53-dependent cell cycle arrest and p53-related CHK2 activation, we assessed chromosome damage by metaphase spread experiment and DNA replication by BrdU incorporation and FACS analysis. Analysis of metaphase cells clearly indicated that irofulven induces chromosome breaks, triradials and quadriradials in A2780 and CAOV3 cells (Fig. 3A). Similarly, chromosome
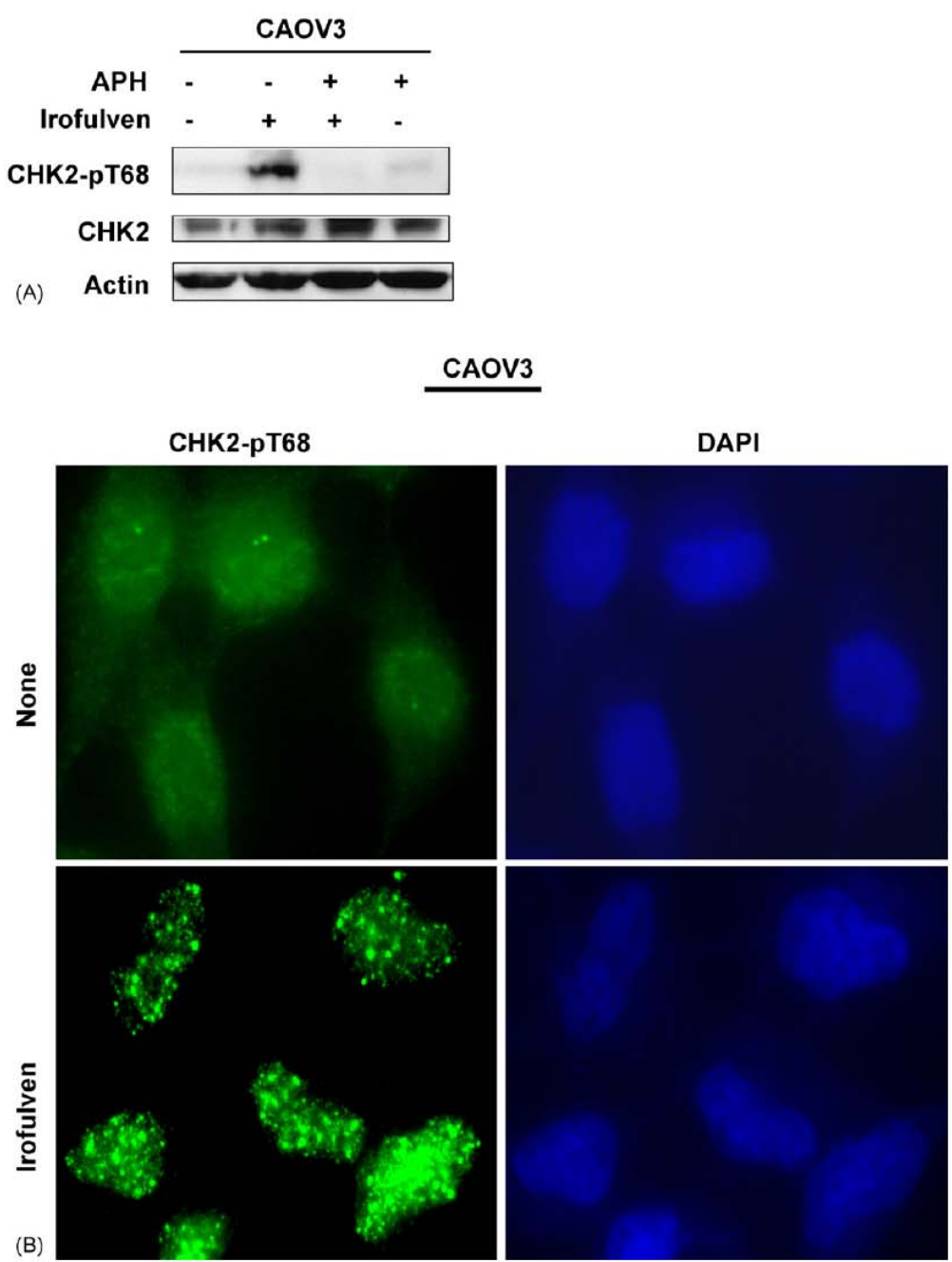

Fig. 4 - CHK2 activation induced by irofulven is DNA replication-dependent. CAOV3 cells were pretreated with aphidicolin (APH, $1 \mu \mathrm{M}, 2 \mathrm{~h}$ ) before being treated with $0.9 \mu \mathrm{M}$ of irofulven for $1 \mathrm{~h}$ followed by $12 \mathrm{~h}$ of incubation in medium containing APH. (A) CHK2 activation was determined by Westem blot analysis with antibody recognizing Thr 68-phosphorylated CHK2. Blots for CHK2 served as the loading control. (B and C) CHK2 foci formation was assessed by immunofluorescent staining. Cells with five or more foci were counted as positive for staining. The percentage of cells with phosphorylated CHK2 foci was exhibited as the mean and standard deviation of triplicate counts of 100 cells. 


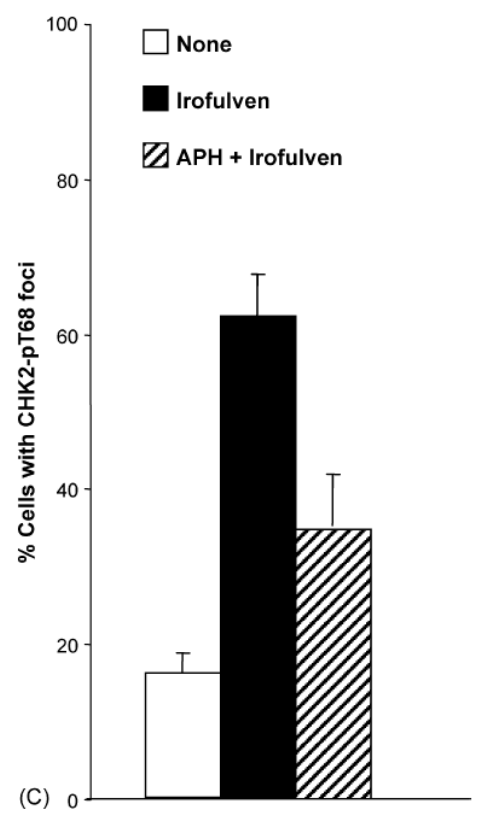

Fig. 4. (Continued).

breaks and radials were also observed in HCT116 p53+/+ and HCT116 p53-/- cells after treatment (data not shown).

Consistent with the observed chromosome damage, DNA synthesis was completely in hibited in A2780 and CAOV3 cells in the presence of irofulven, as determined by BrdU incorporation and FACS analysis (Fig. 3B). Therefore, these data demonstrate that irofulven induces chromosome damage, stalled DNA replication and p53-dependent cell cycle arrest.

\subsection{CHK2 activation induced by irofulven is DNA replication-dependent}

We observed that irofulven induces chromosome aberrations, stalled DNA replication and p53-dependent cell cycle arrest; and CHK2 activation is related to 553 status. We hypothesized that CHK2 might be activated by stalled DNA replication, therefore, it might be replication-dependent and affected by p53-dependent cell cycle arrest. To test this, CAOV3 cells were pretreated with aphidicolin, a specific inhibitor of DNA polymerase $\alpha$ [54]. It was demonstrated that CHK2 activation by irofulven was indeed reduced by aphidicolin treatment (Fig. 4A). Similar reduction of CHK2 activation by aphidicolin was also observed in HCT116 p53-/- cells (data not shown).

Furthermore, we examined the foci formation of phosphorylated CHK2 on Thr 68 using immunofluorescent staining in CAOV3 cells after aphidicolin and irofulven treatment. The results demonstrated that phosphorylated CHK2 forms foci upon irofulven treatment (Fig. 4B), and CHK2 foci formation is reduced by aphidicolin treatment (Fig. 4C). Taken together, these results indicate that CHK2 activation induced by irofulven results from stalled DNA replication and is related to p53-mediated cell cycle arrest.

3.5. $p 53$ or CHK2 activation does not contribute to chemosensitivity to irofulven

The activation and accumulation of p53 after irofulven treatment was observed (Fig. 2). Previous studies conducted in tumor cell lines with wild-type or mutant p53 suggested that the cytotoxicity induced by irofulven is independent of p53 status [55-57]. To confirm these results in an isogenic background, we performed clonogenic assay in HCT116 p53+/+ and p53-/- cells. The results indicated that $\mathrm{p} 53$ status does not affect chemosensitivity to irofulven (Fig. 5A).

Reports have been inconsistent regarding the role that CHK2 plays in apoptosis. To determine whether CHK2 activation modulates chemosensitivity to irofulven, we performed clonogenic survival assays in isogenic vector and dominant-negative CHK2-transfected CAOV3 cells, along with paired isogenic HCT116 $\mathrm{CHK} 2+/+$ and CHK2-/- cells.

First, we established stable CAOV 3 cell lines expressing the HA-tagged dominant-negative CHK2 (CHK2 kinase-dead, CHK2.kd) [45]. The HA-CHK2.kd expression was verified by Western blot analysis with antibodies against HA and CHK2 (Fig. 5B). The effect of CHK2.kd in blocking endogenous CHK2 kinase activity was verified by immunoprecipitation and in vitro kinase assay with GST-CDC25C fusion protein as the substrate. As shown in Fig. $5 \mathrm{C}$, the CHK2 kinase activity induced by irofulven was blocked by CHK2.kd expression. However, when the clonogenic survival assay was carried out in the cell lines, the results indicated that CHK2 did not contribute to irofulven-induced chemosensitivity (Fig. 5D).

To further confirm these findings, the paired, isogenic colon cancer cell line HCT116 CHK2+/+ and its CHK2-knockout cell line HCT116 CHK2-/- [44] were used. The CHK2 knockout status was verified by Western blot analysis (Fig. 5E). Clonogenic survival assay was performed and the results again demonstrated that CHK2 does not contribute to the chemosensitivity to irofulven (Fig. 5F).

3.6. CHK2 activation contributes to the control of $S$ and G2/M cell cycle arrest in response to irofulven-induced DNA damage

To explore the role that CHK2 activation plays in response to irofulven-induced DNA damage, we analyzed the alterations of cell cycle progression in CHK2.kd transfected cells and in CHK2-knockout cells. The radioresistant DNA synthesis (RDS) assay was performed to monitor the $\mathrm{S}$ phase checkpoint. The immunofluorescent staining for phospho-histone H3, a marker for mitosis, and FACS analysis were performed to assess the G2/M checkpoint.

The vector and CHK2.kd-transfected CAOV3 cells were treated with irofulven. The results of the RDS assay demonstrated that DNA synthesis was inhibited in vector-transfected CAOV3 cells after treatment compared with CHK2.kd-transfected cells $(p<0.05$ and $<0.01)$ (Fig. 6A). Similarly, when the DNA synthesis rate was assessed by RDS assay in isogenic HCT116 CHK2+/+ and CHK2-/- cells, more inhibition of DNA synthesis was observed in $\mathrm{CHK} 2+/+$ cells than in $\mathrm{CHK} 2-/-$ cells 

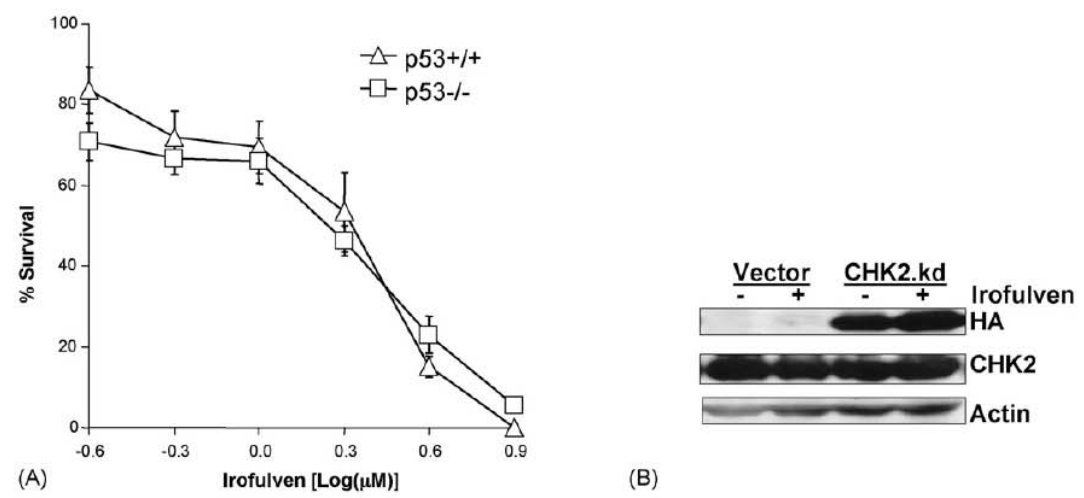

(A) Irofulven $[\log (\mu M)$

(B)
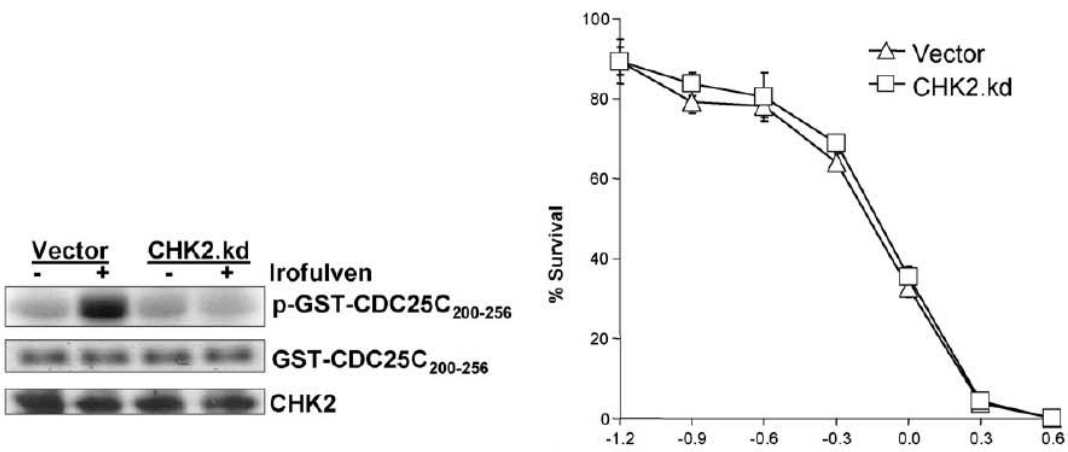

(C)

(D)

Irofulven $[\log (\mu \mathrm{M})]$

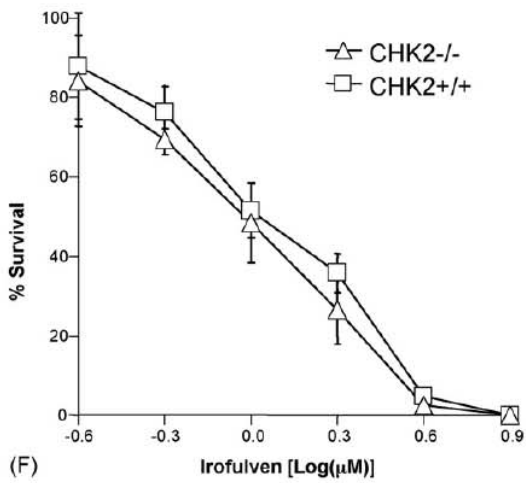

$(\mathrm{F})$

Fig. 5 - p53 or CHK2 activation does not contribute to chemosensitivity to irofulven. Cells were treated with irofulven for $1 \mathrm{~h}$ followed by drug-free incubations. Irofulven-induced chemosensitivity was determined by clonogenic survival assay. The mean and standard deviation of triplicate experiments were presented. (A) The clonogenic survival of HCT116 p53+/+ and p53-/- cells treated with irofulven. The p53 knock-out status of these cells was shown in Fig. 2D. (B and C) Stable transfection of HA-tagged kinase-dead CHK2 (CHK2.kd) into CAOV3 cells. The vector and CHK2.kd-transfected CAOV3 cells were treated with $0.9 \mu \mathrm{M}$ of irofulven for $1 \mathrm{~h}$ followed by $24 \mathrm{~h}$ of drug-free incubation. Westem blot analyses were performed with antibodies against $\mathrm{HA}, \mathrm{CHK} 2$ and actin (B). Immunoprecipitation and in vitro kinase assay were conducted with the antibody against CHK2 and with purified GST-CDC25C ${ }_{200-256}$ fusion protein as the substrate. The amounts of immunoprecipitated $\mathrm{CHK} 2$ and GST-CDC25C $200-256$ substrate in each reaction were verified by Western blot and Coommassie blue staining, respectively (C). (D) The clonogenic survival of vector and CHK2.kd-transfected CAOV3 cells treated with irofulven. (E) The CHK2 status in parental (HCT116 CHK2+/+) and CHK2-knockout (HCT116 CHK2-/-) cells was verified by Westem blot analysis. The asterisk denotes a non-specific band. (F) The clonogenic survival of HCT116 CHK2+/+ and CHK2-1- cells treated with irofulven. 

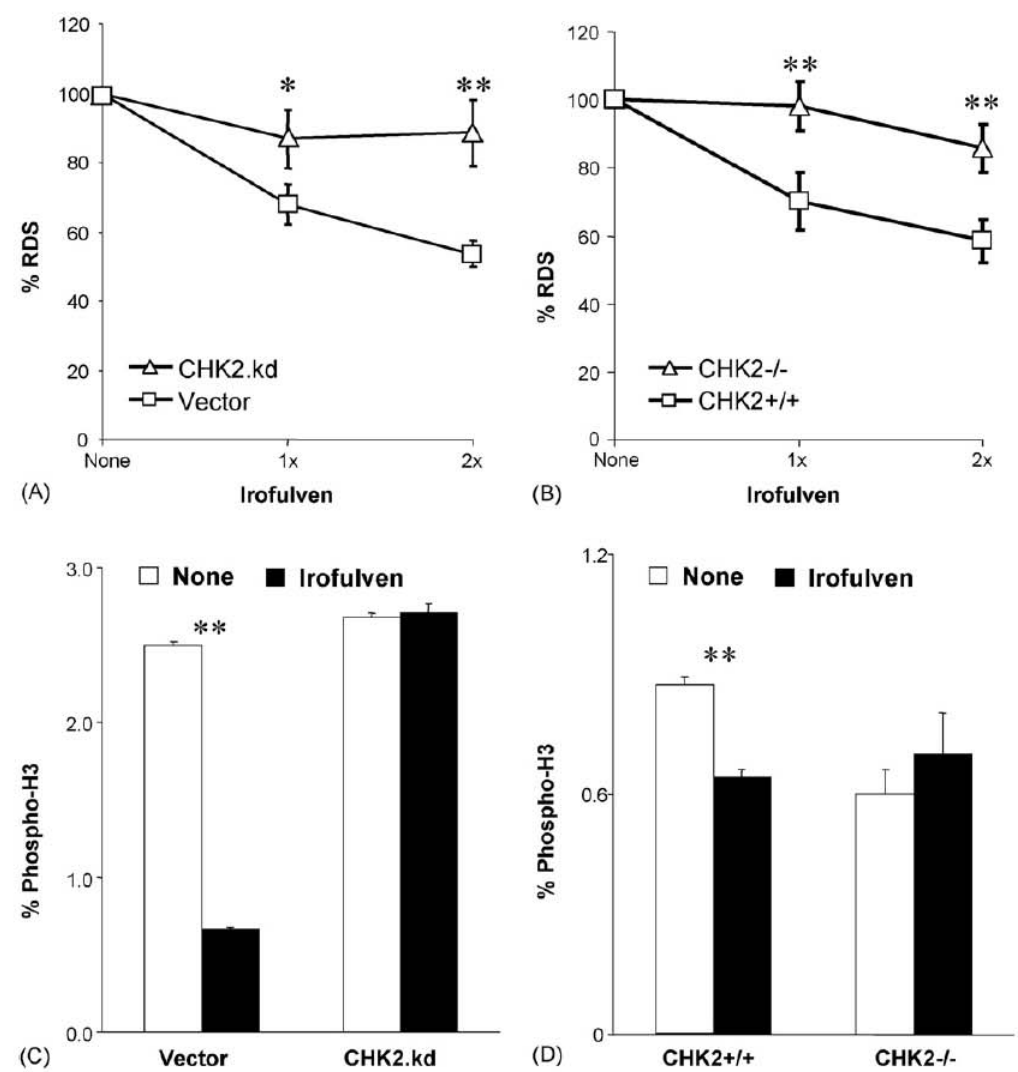

Fig. 6 - CHK2 activation contributes to the control of $\mathrm{S}$ and G2/M arrests after irofulven treatment. The statistical significance was analyzed by Student's t-test and marked as $p<0.05$ and $" p<0.01$. DNA synthesis was determined by RDS assay. The vector and CHK2.kd-transfected CAOV3 cells (A), and HCT116 CHK2+/+ and CHK2-/- cells (B) were treated with $1 \times$ or $2 \times$ IC50 concentration of irofulven for $1 \mathrm{~h}$ followed by $3 \mathrm{~h}$ of drug-free incubation. DNA synthesis rate was presented as the average and standard error of triplicate experiments. The mitotic population of cells was determined by immunofluorescent staining of phosphorylated histone $\mathrm{H} 3$ and flow cytometry. The vector and CHK2.kd-transfected CAOV3 cells (C), and HCT116 CHK2+/+ and CHK2-/- cells (D) were treated with $1 \times$ IC50 concentration of irofulven for $1 \mathrm{~h}$ followed by 1 or $3 \mathrm{~h}$ of drug-free incubation. The percentage of phospho-histone H3-positive cells was exhibited as the mean and standard deviation of triplicate experiments.

after treatment $(p<0.01)$ (Fig. 6B). These results indicate that $\mathrm{CHK} 2$ contributes to the control of $\mathrm{S}$ phase checkpoint in response to irofulven.

The results of FACS analysis of phospho-histone H3 staining demonstrated that $1 \mathrm{~h}$ after treatment, phospho-histone H3positive population decreased from $2.5 \%$ to $0.66 \%$ in vectortransfected CAOV3 cells $(p<0.01)$; while in CHK2.kd-transfected CAOV3 cells, it was unchanged (Fig. 6C). When the phospho-histone H3-positive population was assessed in isogenic CHK2 knock-out cells $3 \mathrm{~h}$ after treatment, it was decreased from $0.87 \%$ to $0.64 \%$ in CHK $2+/+$ cells $(p<0.01)$; while in CHK2 $-/$ - cells, it was slightly increased from $0.6 \%$ to $0.7 \%$ (Fig. 6D). These results suggest that $\mathrm{CHK} 2$ controls the G2/M checkpoint in response to irofulven treatment.
Taken together, these results demonstrate that CHK2 contributes to the control of S and G2/M cell cycle arrests in response to irofulven-induced DNA damage.

\section{Discussion}

In this study, we found that CHK2 activation is replicationdependent and related to 533 status. 553 affects $\mathrm{CHK} 2$ activation by controlling cell cycle arrest and DNA replication in response to the anticancer agent, irofulven. CHK2 activation contributes to the control of cell cycle arrest, but not chemosensitivity.

Tumor suppressors $\mathrm{p} 53$ and $\mathrm{CHK} 2$ are frequently mutated in human cancers [5,6]. Both p53 and CHK2 play important 
roles in cell cycle control, DNA repair and apoptosis [2,5-26]. While many studies have investigated how CHK2 activates and stabilizes p53 [6,17,27-29], the possible role of p53 in regulating CHK2 protein level or activity has not been well studied. Studies conducted in tumor cell lines and tissue specimens have previously demonstrated an inverse correlation between $p 53$ and CHK2 expression [30,31]. However, in our previous and current studies, neither HCT116, HCT116 p53-/- nor p53 wild-type and mutant ovarian cancer cell lines demonstrated a dramatic inverse correlation of $\mathrm{p} 53$ and CHK2 protein levels after irofulven treatment [42]. Instead, we found that p53-deficient cells had enhanced CHK2 activation due to the loss of p53-regulated G1 arrest and stalled DNA replication in response to irofulven-induced DNA damage. In addition, we demonstrated that $\mathrm{CHK} 2$ activation contributes to the control of $\mathrm{S}$ and G2/M checkpoints. Therefore, enhanced CHK2 activation in p53-deficient cells serves as a mechanism to enforce cells arresting at S or G2/M phases in response to irofulven-induced DNA damage.

Previous studies conducted in tumor cell lines with wildtype or mutant $\mathrm{p} 53$ suggested that the cytotoxicity induced by irofulven is independent of p53 status [55-57]. In this study, the chemosensitivity to irofulven was compared in isogenic HCT116 p53+/+ and p53-/- cells. It was found that p53 status does not affect chemosensitivity to irofulven.

In mammalian cells, CHK2 was shown to promote DNA double-strand break repair via homologous recombination (HR) and non-homologous end-joining (NHEJ) by phosphorylating BRCA1 on serine 988 [10-13]. The CHK2 phosphorylation and nuclear foci formation observed in this study suggest that CHK2 was activated and recruited to the DNA damage sites, mediating the DNA repair process. CHK2 has also been implicated in regulating apoptosis [14-26]. However, there are some variations in the results reported. Some studies found that CHK2 is a negative regulator of mitotic catastrophe, and inhibition of CHK2 sensitizes cells to chemotherapy-induced apoptosis $[14,15]$. One report suggested that CHK2 does not affect neuronal cell death induced by topoisomerase I or II inhibitors [20]. Other studies found that CHK2 is involved in apoptosis induction, however there is disagreement with regards to whether it is dependent or independent of p53 [1623]. Given the diverse roles of CHK2 in DNA repair and apoptosis induction, whether CHK2 activation ultimately contributes to chemosensitivity was not evaluated in these studies. Here, we found that the loss or inhibition of CHK2 is not sufficient to render cancer cells more susceptible to irofulven treatment. CHK2 activation only contributes to the control of cell cycle arrests at $\mathrm{S}$ and $\mathrm{G} 2 / \mathrm{M}$ phases in response to irofulven-induced DNA damage.

Recent reports have indicated that ATM and CHK2 are specifically activated by drug (calicheamicin) or radiationinduced DNA double-strand breaks (DSBs), and the level of activation directly correlates to the number of DNA DSBs $[58,59]$. Our previous studies demonstrated that irofulven activates ATM and its targets, NBS1, SMC1, CHK2 and p53 [42]. In this study, we found that irofulven induces chromosome aberrations (breaks and radials), inhibits DNA replication and activates CHK2 in a replication-dependent manner. These data suggest that irofulven causes stalled DNA replication and the generation of DNA DSBs. Further studies are needed to elucidate the mechanisms that lead to stalled DNA replication, and whether proteins that are critical for the HR repair of DNA DSBs will modulate irofulven-induced chemosensitivity.

In summary, this study demonstrated that irofulveninduced CHK2 activation is replication-dependent and related to $\mathrm{p} 53$ status. $\mathrm{p} 53$ affects $\mathrm{CHK} 2$ activation by controlling cell cycle arrest and DNA replication. CHK2 activation contributes to the control of $\mathrm{S}$ and $\mathrm{G} 2 / \mathrm{M}$ cell cycle arrests, but not chemosensitivity. Further characterization of irofulveninduced DNA damage and elucidation of the DNA damage signaling and repair pathways will allow a better understanding of the mechanisms of action involved with irofulven and improved application of this drug in the clinic.

\section{Acknowledgments}

We would like to thank Bert Vogelstein and Fred Bunz (Johns Hopkins University, Baltimore, MD) for generously providing colon cancer cell line HCT116 and its isogenic p53-knockout (HCT116 p53-/-) and CHK2-knockout (HCT116 CHK2-/-) cells; and thank Jann N. Sarkaria (Mayo Clinic and Foundation, Rochester, MN) for generously providing the HA-CHK2.kd and GST-CDC25C $200-256$ plasmids. We also thank Emily Van Laar for helpful discussions and critical reading of the manuscript. This work was supported by NIH grant R03CA107979 (to W.W.).

\section{R E F E R E N C E S}

[1] Sherr CJ. The Pezcoller lecture: cancer cell cycles revisited. Cancer Res 2000;60(14):3689-95.

[2] Zhou BB, Elledge SJ. The DNA damage response: putting checkpoints in perspective. Nature 2000;408(6811):433-9.

[3] Abraham RT. Cell cycle checkpoint signaling through the ATM and ATR kinases. Genes Dev 2001;15(17):2177-96.

[4] Shiloh Y. ATM and related protein kinases: safeguarding genome integrity. Nat Rev Cancer 2003;3(3):155-68.

[5] Levine AJ. p53, the cellular gatekeeper for growth and division. Cell 1997;88(3):323-31.

[6] Bartek J, Lukas J. CHK1 and CHK2 kinases in checkpoint control and cancer. Cancer Cell 2003;3(5):421-9.

[7] Cory S, Adams JM. The Bcl2 family: regulators of the cellular life-or-death switch. Nat Rev Cancer 2002;2(9):647-56.

[8] Vousden KH, Lu X. Live or let die: the cell's response to p53. Nat Rev Cancer 2002;2(8):594-604.

[9] Boddy MN, Shanahan P, McDonald WH, Lopez-Girona A Noguchi E, Yates IJ, et al. Replication checkpoint kinase Cds1 regulates recombinational repair protein Rad60. Mol Cell Biol 2003;23(16):5939-46.

[10] Zhang J, Willers H, Feng Z, Ghosh JC, Kim S, Weaver DT, et al. CHK2 phosphorylation of BRCA1 regulates DNA double-strand break repair. Mol Cell Biol 2004;24(2):708-18.

[11] Lee JS, Collins KM, Brown AL, Lee CH, Chung JH. hCds1mediated phosphorylation of BRCA1 regulates the DNA damage response. Nature 2000;404(6774):201-4.

[12] Wang HC, Chou WC, Shieh SY, Shen CY. Ataxia telangiectasia mutated and checkpoint kinase 2 regulate BRCA1 to promote the fidelity of DNA end-joining. Cancer Res 2006;66(3):1391-400.

[13] Zhuang J, Zhang J, Willers H, Wang H, Chung JH, van Gent DC, et al. Checkpoint kinase 2-mediated phosphorylation of 
BRCA1 regulates the fidelity of nonhomologous endjoining. Cancer Res 2006;66(3):1401-8.

[14] Castedo M, Perfettini JL, Roumier T, Yakushijin K, Horne D, Medema R, et al. The cell cycle checkpoint kinase CHK2 is a negative regulator of mitotic catastrophe. Oncogene 2004;23(25):4353-61.

[15] Castedo M, Perfettini JL, Roumier T, Valent A, Raslova H, Yakushijin K, et al. Mitotic catastrophe constitutes a special case of apoptosis whose suppression entails aneuploidy. Oncogene 2004;23(25):4362-70.

[16] Yang S, Kuo C, Bisi JE, Kim MK. PML-dependent apoptosis after DNA damage is regulated by the checkpoint kinase hCds1/Chk2. Nat Cell Biol 2002;4(11):865-70.

[17] Takai H, Naka K, Okada Y, Watanabe M, Harada N, Saito S, et al. Chk2-deficient mice exhibit radioresistance and defective p53-mediated transcription. EMBO J 2002;21(19):5195-205.

[18] Hirao A, Cheung A, Duncan G, Girard PM, Elia AJ, Wakeham $A$, et al. Chk2 is a tumor suppressor that regulates apoptosis in both an ataxia telangiectasia mutated (ATM)dependent and an ATM-independent manner. Mol Cell Biol 2002;22(18):6521-32.

[19] Jack MT, Woo RA, Hirao A, Cheung A, Mak TW, Lee PW. Chk2 is dispensable for p53-mediated G1 arrest but is required for a latent p53-mediated apoptotic response. Proc Natl Acad Sci USA 2002;99(15):9825-9.

[20] Keramaris E, Hirao A, Slack RS, Mak TW, Park DS. ATM can regulate $\mathrm{p} 53$ and neuronal death independent of $\mathrm{Chk} 2$ in response to DNA damage. J Biol Chem 2003.

[21] Brodsky MH, Weinert BT, Tsang G, Rong YS, McGinnis NM, Golic KG, et al. Drosophila melanogaster MNK/Chk2 and p53 regulate multiple DNA repair and apoptotic pathways following DNA damage. Mol Cell Biol 2004;24(3):1219-31.

[22] Xu J, Xin S, Du W. Drosophila Chk2 is required for DNA damage-mediated cell cycle arrest and apoptosis. FEBS Lett 2001;508(3):394-8

[23] Peters M, DeLuca C, Hirao A, Stambolic V, Potter J, Zhou L, et al. Chk2 regulates irradiation-induced, p53-mediated apoptosis in Drosophila. Proc Natl Acad Sci USA 2002;99(17):11305-10.

[24] Stevens C, Smith L, La Thangue NB. Chk2 activates E2F-1 in response to DNA damage. Nat Cell Biol 2003;5(5):401-9.

[25] Stiewe T, Putzer BM. Role of the p53-homologue p73 in E2F1-induced apoptosis. Nat Genet 2000;26(4):464-9.

[26] Moroni MC, Hickman ES, Denchi EL, Caprara G, Colli E, Cecconi F, et al. Apaf-1 is a transcriptional target for E2F and p53. Nat Cell Biol 2001;3(6):552-8.

[27] Chehab NH, Malikzay A, Appel M, Halazonetis TD. Chk2/ hCds1 functions as a DNA damage checkpoint in G(1) by stabilizing p53. Genes Dev 2000;14(3):278-88.

[28] Shieh SY, Ahn J, Tamai K, Taya Y, Prives C. The human homologs of checkpoint kinases Chk1 and Cds1 (Chk2) phosphorylate p53 at multiple DNA damage-inducible sites. Genes Dev 2000;14(3):289-300.

[29] Hirao A, Kong YY, Matsuoka S, Wakeham A, Ruland J, Yoshida $\mathrm{H}$, et al. DNA damage-induced activation of $\mathrm{p} 53$ by the checkpoint kinase Chk2. Science 2000;287(5459):1824-7.

[30] Tominaga K, Morisaki H, Kaneko Y, Fujimoto A, Tanaka T, Ohtsubo M, et al. Role of human Cds1 (Chk2) kinase in DNA damage checkpoint and its regulation by p53. J Biol Chem 1999;274(44):31463-7.

[31] Shigeishi H, Yokozaki H, Oue N, Kuniyasu H, Kondo T, Ishikawa $\mathrm{T}$, et al. Increased expression of CHK2 in human gastric carcinomas harboring p53 mutations. Int J Cancer 2002;99(1):58-62.

[32] Matsui T, Katsuno Y, Inoue T, Fujita F, Joh T, Niida H, et al. Negative regulation of Chk2 expression by $\mathrm{p} 53$ is dependent on the CCAAT-binding transcription factor NF-Y. J Biol Chem 2004;279(24):25093-100.
[33] MacDonald JR, Muscoplat CC, Dexter DL, Mangold GL, Chen SF, Kelner MJ, et al. Preclinical antitumor activity of 6-hydroxymethylacylfulvene, a semisynthetic derivative of the mushroom toxin illudin S. Cancer Res 1997;57(2): $279-83$.

[34] Sato Y, Kashimoto S, MacDonald JR, Nakano K. In vivo antitumour efficacy of MGI-114 (6hydroxymethylacylfulvene, HMAF) in various human tumour xenograft models including several lung and gastric tumours. Eur J Cancer 2001;37(11):1419-28.

[35] Friedman HS, Keir ST, Houghton PJ, Lawless AA, Bigner DD, Waters SJ. Activity of irofulven (6hydroxymethylacylfulvene) in the treatment of glioblastoma multiforme-derived xenografts in athymic mice. Cancer Chemother Pharmacol 2001;48(5):413-6.

[36] Hidalgo M, Izbicka E, Eckhardt SG, MacDonald JR, Cerna C, Gomez L, et al. Antitumor activity of MGI 114 (6hydroxymethylacylfulvene, HMAF), a semisynthetic derivative of illudin S, against adult and pediatric human tumor colony-forming units. Anticancer Drugs 1999;10(9):837-44.

[37] Britten CD, Hilsenbeck SG, Eckhardt SG, Marty J, Mangold G, MacDonald JR, et al. Enhanced antitumor activity of 6hydroxymethylacylfulvene in combination with ininotecan and 5-fluorouracil in the HT29 human colon tumor xenograft model. Cancer Res 1999;59(5):1049-53.

[38] Murgo A, Cannon DJ, Blatner G, Cheson BD. Clinical trials referral resource. Clinical trials of MGI-114. Oncology (Huntingt) 1999;13(2). p. 233, 237, 238.

[39] Kelner MJ, McMorris TC, Rojas RJ, Trani NA, Velasco TR, Estes LA, et al. Enhanced antitumor activity of irofulven in combination with antimitotic agents. Invest New Drugs 2002;20(3):271-9.

[40] Senzer N, Arsenau J, Richards D, Berman B, MacDonald JR, Smith S. Irofulven demonstrates clinical activity against metastatic hormone-refractory prostate cancer in a phase 2 single-agent trial. Am J Clin Oncol 2005;28(1):36-42.

[41] Woo MH, Peterson JK, Billups C, Liang H, Bjornsti MA, Houghton PJ. Enhanced antitumor activity of irofulven in combination with irinotecan in pediatric solid tumor xenograft models. Cancer Chemother Pharmacol 2005;55(5):411-9.

[42] Wang J, Wiltshire T, Wang Y, Mikell C, Burks J, Cunningham C, et al. ATM-dependent CHK2 activation induced by anticancer agent, irofulven. J Biol Chem 2004;279(38):39584-92.

[43] Bunz F, Dutriaux A, Lengauer C, Waldman T, Zhou S, Brown JP, et al. Requirement for $\mathrm{p} 53$ and p21 to sustain G2 arrest after DNA damage. Science 1998;282(5393): 1497-501.

[44] Jallepalli PV, Lengauer C, Vogelstein B, Bunz F. The Chk2 tumor suppressor is not required for $\mathrm{p} 53$ responses in human cancer cells. J Biol Chem 2003.

[45] Busby EC, Leistritz DF, Abraham RT, Karnitz LM, Sarkaria JN. The radiosensitizing agent 7-hydroxystaurosporine (UCN-01) inhibits the DNA damage checkpoint kinase hChk1. Cancer Res 2000;60(8):2108-12.

[46] Wang W, Waters SJ, MacDonald JR, Roth C, Shentu S, Freeman J, et al. Irofulven (6-hydroxymethylacylfulvene, MGI 114)-induced apoptosis in human pancreatic cancer cells is mediated by ERK and JNK kinases. Anticancer Res 2002;22(2A):559-64.

[47] Xu B, Kim S, Kastan MB. Involvement of Brca1 in S-phase and G(2)-phase checkpoints after ionizing irradiation. Mol Cell Biol 2001;21(10):3445-50.

[48] Matsuoka S, Rotman G, Ogawa A, Shiloh Y, Tamai K, Elledge SJ. Ataxia telangiectasia-mutated phosphorylates Chk2 in vivo and in vitro. Proc Natl Acad Sci USA 2000;97(19):10389-94. 
[49] Melchionna R, Chen XB, Blasina A, McGowan CH. Threonine 68 is required for radiation-induced phosphorylation and activation of Cds1. Nat Cell Biol 2000;2(10):762-5.

[50] Ahn JY, Schwarz JK, Piwnica-Worms H, Canman CE. Threonine 68 phosphorylation by ataxia telangiectasia mutated is required for efficient activation of Chk2 in response to ionizing radiation. Cancer Res 2000;60(21):5934-6.

[51] Lee CH, Chung JH. The hCds1 (Chk2)-FHA domain is essential for a chain of phosphorylation events on hCds1 that is induced by ionizing radiation. J Biol Chem 2001;276(32):30537-41.

[52] Xu X, Tsvetkov LM, Stern DF. Chk2 activation and phosphorylation-dependent oligomerization. Mol Cell Biol 2002;22(12):4419-32.

[53] Ward IM, Wu X, Chen J. Threonine 68 of Chk2 is phosphorylated at sites of DNA strand breaks. J Biol Chem 2001;276(51):47755-8.

[54] Huberman JA. New views of the biochemistry of eucaryotic DNA replication revealed by aphidicolin, an unusual inhibitor of DNA polymerase alpha. Cell 1981;23(3):647-8.
[55] Izbicka E, Davidson K, Lawrence R, Cote R, MacDonald JR, Von Hoff DD. Cytotoxic effects of MGI 114 are independent of tumor p53 or p21 expression. Anticancer Res 1999;19(2A):1299-307.

[56] Poindessous V, Koeppel F, Raymond E, Comisso M, Water SJ, Larsen AK. Marked activity of irofulven toward human carcinoma cells: comparison with cisplatin and ecteinascidin. Clin Cancer Res 2003;9(7):2817-25.

[57] Serova M, Calvo F, Lokiec F, Koeppel F, Poindessous V, Larsen AK, et al. Characterizations of irofulven cytotoxicity in combination with cisplatin and oxaliplatin in human colon, breast, and ovarian cancer cells. Cancer Chemother Pharmacol 2006;57(4):491-9.

[58] Ismail IH, Nystrom S, Nygren J, Hammarsten O. Activation of ataxia telangiectasia mutated by DNA strand break-inducing agents correlates closely with the number of DNA double strand breaks. J Biol Chem 2005;280(6): 4649-55.

[59] Buscemi G, Perego P, Carenini N, Nakanishi M, Chessa L, Chen J, et al. Activation of ATM and Chk2 kinases in relation to the amount of DNA strand breaks. Oncogene 2004;23(46):7691-700. 


\section{Appendix 3}

\section{ATM-dependent CHK2 Activation Induced by Anticancer Agent, Irofulven}

This manuscript was published in The Journal of Biological Chemistry 


\title{
ATM-dependent CHK2 Activation Induced by Anticancer Agent, Irofulven*
}

\author{
Jian Wangł, Timothy Wiltshire§, Yutian Wangł, Carmenza Mikellł, Julian Burks§, \\ Cynthia Cunningham $\S$, Emily S. Van Laarף, Stephen J. Waters», Eddie Reed $\$$, \\ and Weixin Wang $\$ \S \|$ \\ From the $\doteqdot$ Mary Babb Randolph Cancer Center, and §Department of Microbiology, Immunology, and Cell Biology, \\ West Virginia University, Morgantown, West Virginia 26506 and $\uparrow M G I$ Pharma, Inc., Bloomington, Minnesota 55437
}

Irofulven (6-hydroxymethylacylfulvene, HMAF, MGI 114) is one of a new class of anticancer agents that are semisynthetic derivatives of the mushroom toxin illudin S. Preclinical studies and clinical trials have demonstrated that irofulven is effective against several tumor types. Mechanisms of action studies indicate that irofulven induces DNA damage, MAPK activation, and apoptosis. In this study we found that in ovarian cancer cells, CHK2 kinase is activated by irofulven while CHK1 kinase is not activated even when treated at higher concentrations of the drug. By using GM00847 human fibroblast expressing tetracycline-controlled, FLAG-tagged kinase-dead ATR (ATR.kd), it was demonstrated that ATR kinase does not play a major role in irofulveninduced CHK2 activation. Results from human fibroblasts proficient or deficient in ATM function (GM00637 and GM05849) indicated that CHK2 activation by irofulven is mediated by the upstream ATM kinase. Phosphorylation of ATM on Ser ${ }^{1981}$, which is critical for kinase activation, was observed in ovarian cancer cell lines treated with irofulven. RNA interference results confirmed that CHK2 activation was inhibited after introducing siRNA for ATM. Finally, experiments done with human colon cancer cell line HCT116 and its isogenic CHK2 knockout derivative; and experiments done by expressing kinase-dead CHK2 in an ovarian cancer cell line demonstrated that CHK2 activation contributes to irofulven-induced $S$ phase arrest. In addition, it was shown that NBS1, SMC1, and p53 were phosphorylated in an ATM-dependent manner, and p53 phosphorylation on serine 20 is dependent on CHK2 after irofulven treatment. In summary, we found that the anticancer agent irofulven, activates the ATM-CHK2 DNA damage-signaling pathway, and CHK2 activation contributes to $S$ phase cell cycle arrest induced by irofulven.

Irofulven $^{1}$ (6-hydroxymethylacylfulvene, HMAF, MGI 114) is one of a new class of anticancer agents that are analogs of

* The costs of publication of this article were defrayed in part by the payment of page charges. This article must therefore be hereby marked payment of page charges. This article must therefore be hereby marked "advertisement" in
indicate this fact.

|| To whom correspondence should be addressed. Tel.: 304-293-2243; Fax: 304-293-4667; E-mail: wwang@hsc.wvu.edu.

${ }^{1}$ The abbreviations used are: irofulven, 6 -hydroxymethylacylfulvene; CHK1, checkpoint kinase 1; CHK2, checkpoint kinase 2; ATM, ataxia telangiectasia-mutated protein; ATR, ATM-RAD3-related; NBS1, Nijmegen breakage syndrome 1; SMC1, structural maintenance of chrojmegen breakage syndrome 1; SMC1, structural maintenance of chro-
mosome 1; BRCA1, breast cancer 1; MDM2, murine double minute 2; PBS, phosphate-buffered saline; IR, ionizing radiation; siRNA, small interfering RNA; BSA, bovine serum albumin; FITC, fluorescein iso- mushroom-derived illudin toxins. Preclinical studies and clinical trials have demonstrated that irofulven is effective against several tumor types (1-17). Studies of mechanisms of irofulven action suggest that it induces DNA damage, MAP kinase activation and apoptosis (18-20). It is also suggested that irofulven-elicited DNA lesions are mainly repaired by transcriptioncoupled nucleotide excision repair (TC-NER) (21).

In response to DNA damage, the cell evokes signal transduction pathways to arrest at $\mathrm{G}_{1} / \mathrm{S}, \mathrm{S}$, or $\mathrm{G}_{2} / \mathrm{M}$ checkpoints, allowing time to deal with the insult $(22,23)$. It has been well documented that DNA damage activates ATM (ataxia telangiectasia-mutated), and/or ATR (ATM-RAD3-related) kinases, two apical protein kinases of the DNA damage response pathways. ATM and ATR phosphorylate downstream effector kinases, CHK1 and CHK2. It is generally believed that ATM is the kinase mainly responding to ionizing radiation (IR)-induced DNA double strand breaks, while ATR responds to the formation of DNA adducts and stalled replication induced by UV, genotoxic drugs, and radiation (23-29).

ATM phosphorylates NBS1 on $\mathrm{Ser}^{343}$ and activates its function in forming the MRE11-RAD50-NBS1 complex and S phase checkpoint control (30-32). ATM phosphorylates SMC1 on $\mathrm{Ser}^{957}$ and $\mathrm{Ser}^{966}$. Activated SMC1 plays a critical role in S phase checkpoint control and radiosensitivity (33, 34). ATM also phosphorylates MDM2 on $\mathrm{Ser}^{395}$, indirectly regulating p53 activity (35). Both ATM and CHK2 phosphorylate BRCA1 (3638). BRCA1 plays an important role in $\mathrm{S}$ and $\mathrm{G}_{2} / \mathrm{M}$ checkpoint control (39-41).

ATM phosphorylates CHK2 on $\mathrm{Thr}^{68}$ leading to CHK2 kinase activation (42-46), while both ATM and ATR phosphorylate CHK1 on $\operatorname{Ser}^{317}$ and Ser $^{345}$ resulting in its activation (29, 47-49). ATM and ATR phosphorylate p53 on $\operatorname{Ser}^{15}(23,50-53)$, and CHK1 and CHK2 phosphorylate p53 on $\operatorname{Ser}^{20}(54-59)$, leading to its accumulation and activation. Activation of p53 initiates cell cycle arrest- and DNA repair-related genes such as p21, GADD45, and $14-3-3 \delta$ and leads to $\mathrm{G}_{1}$ and $\mathrm{G}_{2}$ arrest (22, $23,54-56,60,61)$. Activation of p53 also regulates the expression of a plethora of apoptosis-related genes resulting in p53dependent apoptosis (62-64). Activation of CHK1 and CHK2 also regulates $\mathrm{S}$ phase by phosphorylating CDC25A (65-72), or the $\mathrm{G}_{2} / \mathrm{M}$ transition by phosphorylating CDC25C $(29,42,68$, $71,73-76$ ). However, controversy exists regarding the role of CHK2 in cell cycle regulation and p53 phosphorylation. Recent studies indicate that $\mathrm{CHK} 2$ is dispensable in radiation-induced $\mathrm{G}_{1}$ and $\mathrm{G}_{2} / \mathrm{M}$ arrest, and CHK1 and CHK2 are unlikely to be the regulators of p53 (77-79). It has also been shown that cells

thiocyanate; MAPK, mitogen-activated protein kinase; GFP, green fluorescent protein. 
from CHK2 knockout mice have normal $\mathrm{S}$ phase and $\mathrm{G}_{2} / \mathrm{M}$ transition $(56,57)$.

In this study, we found that the anticancer agent, irofulven, activates ATM-CHK2 DNA damage-signaling pathway, and activates NBS1, SMC1, and p53 in an ATM-dependent manner. Irofulven also induces CHK2-dependent p53 phosphorylation on $\mathrm{Ser}^{20}$. This $\mathrm{CHK} 2$ activation contributes to irofulven-induced $\mathrm{S}$ phase arrest.

\section{EXPERIMENTAL PROCEDURES}

Cell Culture-All cell lines were maintained in various media supplemented with $10 \%$ fetal bovine serum in a $37^{\circ} \mathrm{C}$ incubator with $5 \%$ $\mathrm{CO}_{2}$ atmosphere. Human ovarian cancer cell lines A2780, A2780/CP70, CAOV3, OVCAR3, and SKOV3 were cultured in RPMI1640. Human colon cancer cell line HCT116 and its isogenic CHK2 knockout derivative HCT116 CHK2-/- (78) (generously provided by Prof. Bert Vogelstein, Johns Hopkins University, Baltimore, MD) were cultured in McCoy's 5A medium. The SV40-transformed human normal fibroblast GM00637, the AT (ataxia telangiectasia) fibroblast GM05849 and its parental non-transformed AT fibroblast GM05823 (Coriell Institute, Camden, NJ) were grown in DMEM. The ATM-complemented AT fibroblast (AT22IJE-T-pEBS7-YZ5, generously provided by Prof. Yosef Shiloh, Tel Aviv University, Israel) (80) was grown in Dulbecco's modified Eagle's medium with $100 \mu \mathrm{g} / \mathrm{ml}$ hygromycin (Invitrogen, Carlsbad, CA). GM00847 human fibroblast-expressing tetracycline-controlled, FLAGtagged kinase-dead ATR (ATR.kd) (81) (generously provided by Drs. Stuart L. Schreiber and Shlomo Handeli of the Fred Hutchinson Cancer Research Center, Seattle, WA) was grown in DMEM supplemented with $400 \mu \mathrm{g} / \mathrm{ml}$ of G418 (Invitrogen). For ATR.kd induction, cells were treated with $1.5 \mu \mathrm{g} / \mathrm{ml}$ of doxycycline (Sigma) for $48 \mathrm{~h}$. For UV treatment in control experiments, cells were treated with $50 \mathrm{~J} / \mathrm{m}^{2}$ of UV light in Stratalinker 2400 (Stratagene, La Jolla, CA) followed by one additional hour of incubation. To inhibit CDC25A degradation, proteasome inhibitor LLnL ( $N$-Acetyl-Leu-Leu-Norleu-al) (Sigma) $(50 \mu \mathrm{M})$ were added to cells $30 \mathrm{~min}$ before irofulven treatment.

Colonogenic Survival Assay-The $\mathrm{IC}_{50}$ concentration of irofulven in ovarian cancer cell lines and the colon cancer cell line HCT116 was determined by colonogenic survival assay. Cells were plated in 6-well plates overnight in complete medium. Cells were treated with different concentrations of irofulven for $1 \mathrm{~h}$. Medium was then replaced with fresh drug-free medium and incubated for 7-10 days. Colonies were stained with PBS containing $0.04 \%$ crystal violet and $0.5 \%$ paraformaldehyde for about $10 \mathrm{~min}$. Staining liquid was aspirated and colonies counted.

Western Blotting-Western blot was performed as described previously $(20)$. Briefly, cell lysates were prepared in cold immunoprecipitation buffer $(10 \mathrm{mM}$ Tris, $\mathrm{pH} 7.4,1 \%$ Triton X-100, $0.5 \%$ Nonidet P-40, $150 \mathrm{~mm} \mathrm{NaCl}, 30 \mathrm{mM}$ sodium fluoride, $40 \mathrm{mM} \beta$-glycerophosphate, 20 $\mathrm{mM}$ sodium pyrophosphate, $1 \mathrm{mM}$ EDTA, $1 \mathrm{mM}$ EGTA, and $0.2 \mathrm{mM}$ phenylmethylsulfonyl fluoride). Total cellular protein $(40 \mu \mathrm{g})$ was electrophoresed in SDS-PAGE gels and transferred to Immobilon-P membranes (Millipore, Bedford, MA). Western blot protein detection was performed using the ECL kit (Amersham Biosciences) according to the manufacturer's recommendations. Monoclonal antibodies against actin and FLAG were purchased from Sigma. Monoclonal antibody against PARP and polyclonal antibodies against ATR, SMC1, NBS1, CDC25A, p53, and phosphorylated p53 on Ser $^{15}$ were purchased from Santa Cruz Biotechnology (Santa Cruz, CA). Polyclonal antibodies against phosphorylated CHK1 on $\mathrm{Ser}^{345}$, phosphorylated CHK2 on $\mathrm{Thr}^{68}$, phosphorylated p53 on $\mathrm{Ser}^{20}$, CHK1 and CHK2 were purchased from Cell Signaling Technology (Beverly, MA). Polyclonal antibody against phosphorylated ATM on Ser ${ }^{1981}$ was purchased from Rockland Immunochemicals (Gilbertsville, PA). Polyclonal antibody for ATM was purchased from Oncogene Research Products (San Diego, CA). Polyclonal antibodies against phosphorylated NBS1 on $\mathrm{Ser}^{343}$ and phosphorylated SMC1 on $\operatorname{Ser}^{957}$ were purchased from Novus Biologicals (Littleton, CO).

BrdU Pulse Labeling and Flow Cytometry-Cells were prelabeled with $10 \mu \mathrm{M}$ BrdU (Roche Applied Science, Indianapolis, IN) for $1 \mathrm{~h}$ before treatment with irofulven for $1 \mathrm{~h}$. After drug removal, cells were incubated in drug-free medium and harvested by trypsinization at different time points. Cells were washed once with cold PBS and fixed in $75 \%$ ethanol/PBS. Cells were then washed with $1 \% \mathrm{BSA} / \mathrm{PBS}$ and resuspended in $2 \mathrm{~N} \mathrm{HCl} / 0.5 \%$ Triton X-100. After incubating for $30 \mathrm{~min}$ at room temperature, the cells were washed twice with $1 \% \mathrm{BSA} / \mathrm{PBS}$ and resuspended in $0.2 \mathrm{ml}$ of $1 \% \mathrm{BSA} / \mathrm{PBS}$. Anti-BrdU antibody $(20 \mu \mathrm{l})$ (BD PharMingen, San Diego, CA) was added to each cell suspension.
After incubating in the dark for $30 \mathrm{~min}$ at room temperature, cells were centrifuged and resuspended in PBS containing $10 \mu \mathrm{g} / \mathrm{ml}$ propidium iodide, $20 \mu \mathrm{g} / \mathrm{ml}$ RNase A, $0.1 \%$ sodium citrate, and $0.1 \%$ Triton X-100. Cells were analyzed by FACSCalibur (BD Biosciences). Cell cycle distributions among BrdU-positive cells were analyzed by ModFit v3.0 software (Verity, Topsham, ME).

RNA Interference-The sense and antisense oligonucleotides for small interfering RNA (siRNA) were synthesized and annealed by Dharmacon (Lafayette, CO). The sequences of two double-stranded siRNAs for ATM are CATCTAGATCGGCATTCAG and TGGTGCTATTTACGGAGCT. The siRNA sequence for bacterial green fluorescence protein (GFP) gene is TGGAAGCGTTCAACTAGCA. A2780 cells were transfected twice within a 24 -h period starting at $40 \%$ confluence with $100 \mathrm{nM}$ final concentrations of siRNAs for ATM (two ATM siRNAs were pooled) and GFP using Oligofectamine (Invitrogen) according to the manufacturer's recommendations. Twenty-four hours after transfection, cells were treated with irofulven for $1 \mathrm{~h}$, washed, and incubated in drug-free media, and harvested $12 \mathrm{~h}$ later. Cell lysates were prepared for Western blot as described above.

Transfection of Kinase-dead CHK2 and Flow Cytometry-Ovarian cancer cell line CAOV3 was transfected twice within a 24-h period with vector or HA-tagged kinase-dead CHK2 (82) (generously provided by Dr. Jann Sarkaria of the Mayo Clinic and Foundation, Rochester, MN) using FuGENE 6 (Roche Applied Science, Indianapolis, IN) according to the manufacturer's recommendations. Forty-eight hours after transfection, cells were treated with irofulven for $1 \mathrm{~h}$, washed, and incubated in drug-free media, and harvested $12 \mathrm{~h}$ later. Cells were then washed with PBS and fixed in 70\% ethanol. After washing twice with PBS, cells were permeabilized with $0.25 \%$ Triton X-100 in PBS on ice for $15 \mathrm{~min}$, then washed with $1 \%$ BSA/PBS, and the cell pellet was suspended in $100 \mu \mathrm{l}$ of $1 \% \mathrm{BSA} / \mathrm{PBS}$ containing $1 \mu \mathrm{g}$ of FITC-conjugated anti-HA antibody (Roche Applied Science) for $3 \mathrm{~h}$ at room temperature. Cells were washed three times in PBS and then resuspended in PBS containing $10 \mu \mathrm{g} / \mathrm{ml}$ of propidium iodide and $20 \mu \mathrm{g} / \mathrm{ml}$ of RNase A. Cells were analyzed by FACSCalibur (BD Biosciences), and cell cycle distributions for propidium iodide and FITC-positive cells were analyzed by ModFit v3.0 software (Verity, Topsham, ME).

\section{RESULTS}

Irofulven Activates CHK2 Kinase in Ovarian Cancer Cells-To assess irofulven cytotoxicity, ovarian cancer cell lines were treated with different concentrations of irofulven for $1 \mathrm{~h}$, then the drug was removed, and colonogenic formation assays were performed. The $\mathrm{IC}_{50}$ concentrations obtained for ovarian cancer cell lines (A2780, A2780/CP70, CAOV3, SKOV3, and OVCAR3) ranged from 0.7 to $2.3 \mu \mathrm{M}$.

In response to DNA damage, CHK2 kinase is phosphorylated at $\mathrm{Thr}^{68}$, which is critical for CHK2 activation $(42-44,46,83)$. To investigate the DNA damage response pathway that might be activated by irofulven-induced DNA damage, ovarian cancer cells were treated with the $1 \times \mathrm{IC}_{50}$ concentration of irofulven for $1 \mathrm{~h}$, and incubated for $24 \mathrm{~h}$ after drug treatment. Western blot assay was performed with antibody recognizing the phosphorylated form of CHK2 on $\mathrm{Thr}^{68}$. As indicated in Fig. 1A, CHK2 kinase was activated by irofulven in all ovarian cancer cell lines. The activation of CHK2 kinase by irofulven was time and dose-dependent (Fig. 1, $B$ and $C$ ).

CHK1 Kinase Is Not Activated by Irofulven-Because CHK1 kinase primarily responds to UV and drug-induced DNA damage (23-29), it was critical to test whether CHK1 was activated by irofulven-induced DNA damage. Western blot assay was performed with antibody recognizing phosphorylated CHK1 on $\mathrm{Ser}^{345}$. As shown in Fig. 2, CHK1 was not activated by irofulven in all four cell lines tested, even when cells were treated with $3 \times \mathrm{IC}_{50}$ concentrations, a treatment resulting in $\sim 90 \%$ cell killing. A2780 treated with $50 \mathrm{~J} / \mathrm{m}^{2}$ of UV light served as positive control for antibody against phosphorylated CHK1 (Fig. 2).

Next, we examined whether CHK1 was activated in the absence of CHK2 expression. Isogenic human colon cancer cell line HCT116 and its CHK2 knockout derivative (78) were treated with $1 \times \mathrm{IC}_{50}$ concentration of irofulven for $1 \mathrm{~h}$ followed 
A.

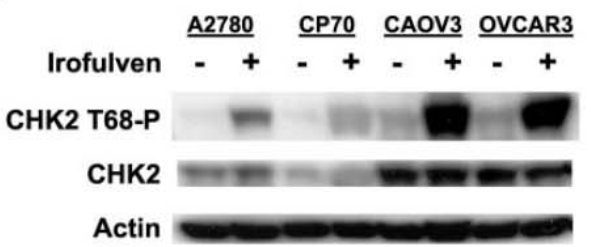

B.

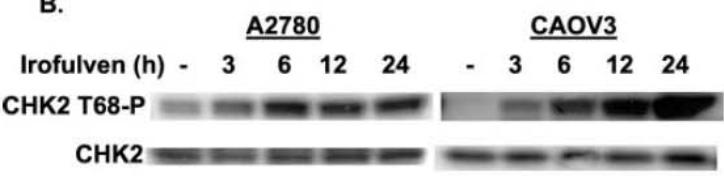

C.

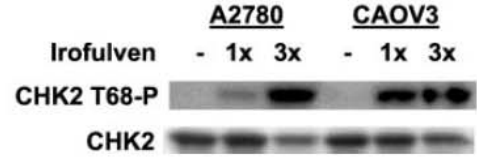

FIG. 1. CHK2 activation by irofulven in ovarian cancer cell lines. Antibody recognizing the phosphorylated form of CHK2 kinase on $\mathrm{Thr}^{68}$ was used to determine the activated CHK2. Blots for CHK2 and actin served as loading control. $A$, cells were treated with $1 \times \mathrm{IC}_{50}$ concentration of irofulven for $1 \mathrm{~h}$ followed by additional $24 \mathrm{~h}$ of incubation. $B$, cells were treated as above followed by different incubation time. $C$, cells were treated with $1 \times$ or $3 \times \mathrm{IC}_{50}$ concentration of irofulven for $1 \mathrm{~h}$ followed by additional $24 \mathrm{~h}$ of incubation.

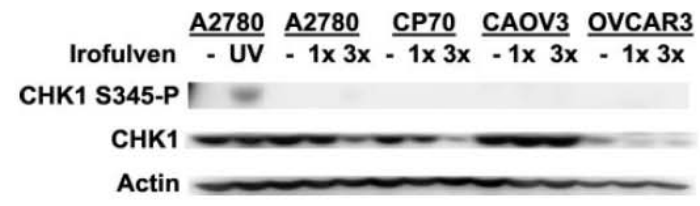

FIG. 2. CHK1 is not activated by irofulven. Ovarian cancer cell lines were treated with $1 \times$ or $3 \times \mathrm{IC}_{50}$ concentration of irofulven for $1 \mathrm{~h}$ followed by additional $24 \mathrm{~h}$ of incubation. Western blot was performed with antibody recognizing phosphorylated CHK1 on $\mathrm{Ser}^{345}$. Extracts from UV $\left(50 \mathrm{~J} / \mathrm{m}^{2}\right)$-treated A2780 cells were loaded as positive control for CHK1 activation. Blots for CHK1 and actin served as loading control.

by an additional 12- or 24-h incubation in drug-free media. Western blot analyses were performed with antibodies against phosphorylated CHK1 on Ser ${ }^{345}$, CHK1, CHK2, and actin. As shown in Fig. $3 A$, CHK1 phosphorylation was not detected in CHK $2+/+$ or CHK $2-/-$ cells. A2780 treated with $50 \mathrm{~J} / \mathrm{m}^{2}$ of UV light served as positive control for CHK1 activation. The Western blot for CHK2 confirmed that CHK2 expression is absent in CHK2-/- cells (Fig. 3A). Further, to demonstrate that the CHK1 activation pathway is intact in these cells, parental HCT116 $(\mathrm{CHK} 2+/+)$ cells were treated with $50 \mathrm{~J} / \mathrm{m}^{2}$ of UV light, and Western blot results indicated that the pathway leading to CHK1 phosphorylation is indeed intact (Fig. $3 B$ ). This is consistent with the finding that CHK1 can be activated when these cells were treated with ionizing radiation (78).

CHK2 Activation by Irofulven Is Not Mediated by ATR-Both ATM and ATR have been reported to activate CHK2 in response to DNA damage $(28,42-46,84,85)$. To determine the possible involvement of ATR in irofulven-induced CHK2 activation, GM00847 human fibroblasts expressing tetracyclinecontrolled, FLAG-tagged kinase-dead ATR (ATR.kd) (81) were used. Both doxycycline-induced and un-induced cells were
A.

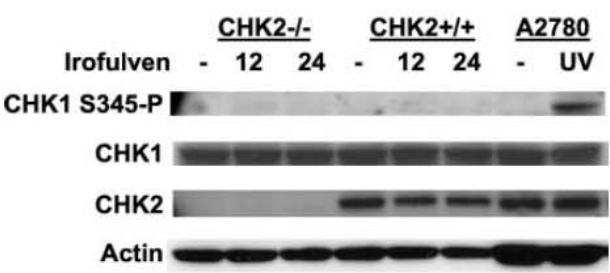

B.

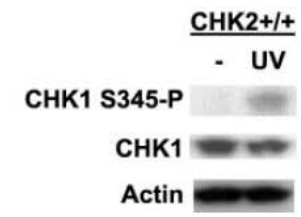

FIG. 3. CHK1 is not activated by irofulven in the absence of CHK2 expression. A, human colon cancer cell line HCT116 and its CHK2 knockout derivative were treated with $1 \times \mathrm{IC}_{50}$ concentration of irofulven for $1 \mathrm{~h}$ followed by an additional 12 or $24 \mathrm{~h}$ of incubation. CHK1 activation was observed by Western blot with antibody recognizing the phosphorylated form of CHK1 on Ser ${ }^{345}$. Western blots for CHK1 and actin served as loading control. The blot for CHK2 indicated the CHK2 status in parental and CHK2 knockout cells. A2780 treated with $50 \mathrm{~J} / \mathrm{m}^{2}$ of UV light served as positive control for antibody against phosphorylated CHK1 on Ser345. B, parental HCT116 $(\mathrm{CHK} 2+/+)$ cells were treated with $50 \mathrm{~J} / \mathrm{m}^{2}$ of UV light, CHK1 activation was determined by Western blot with anti-phospho-CHK1 antibody, and the blots for CHK1 and actin were shown as the loading control.

treated with irofulven. As shown in Fig. 4A, the FLAG-tagged, kinase-dead ATR was strongly induced by $1.5 \mu \mathrm{g} / \mathrm{ml}$ of doxycycline for $48 \mathrm{~h}$ as determined by Western blot with anti-FLAG antibody. However, under the same conditions, CHK2 activation by irofulven $(8 \mu \mathrm{M}, 1 \mathrm{~h}$ treatment followed by $12 \mathrm{~h}$ of incubation) was apparent. There actually was some increase of phosphorylated CHK2 in cells expressing kinase-dead ATR after irofulven treatment (Fig. 4A). To confirm that the doxycycline-induced kinase-dead ATR was functional, the doxycycline-treated and untreated cells were exposed to $50 \mathrm{~J} / \mathrm{m}^{2}$ of UV light. As shown in Fig. 4B, CHK1 kinase was strongly activated by UV in un-induced cells, but the induced kinase-dead ATR greatly blocked CHK1 activation by UV irradiation, indicating that ATR.kd was functional.

CHK2 Activation by Irofulven Is Dependent on ATM-To determine the role that ATM might play in irofulven-induced CHK2 activation, Western blot assay was carried out with cellular extracts from ATM-deficient and proficient cell lines treated with irofulven. As shown in Fig. $5 \mathrm{~A}$, CHK2 was activated by irofulven in a concentration-dependent manner in the SV40-transformed normal human fibroblast GM00637. In contrast, under the same irofulven treatment conditions, CHK2 was not activated in SV40-transformed human AT (ataxia telangiectasia) fibroblast, GM05849, and its parental non-transformed AT fibroblast, GM05823, which are known to be deficient in ATM function (Fig. 5B). As a positive control, the CHK2 activation was observed in the ATM-complemented AT fibroblast (AT22IJE-T-pEBS7-YZ5) (80) (Fig. 5B). Taken together, these results indicate that the CHK2 activation by irofulven is dependent on the ATM status.

DNA damage induces rapid autophosphorylation of ATM on serine 1981. This ATM autophosphorylation causes dimer dissociation and initiates ATM kinase activity (86). To further study the involvement of ATM kinase activation in irofulveninduced CHK2 activation, A2780 and CAOV3 ovarian cancer cells were treated with irofulven for $1 \mathrm{~h}$ followed by $24 \mathrm{~h}$ of 
A.

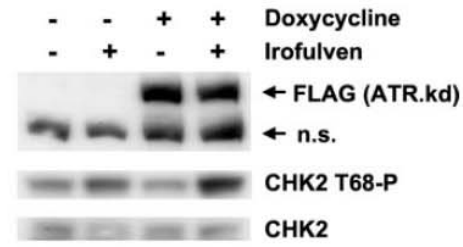

B.

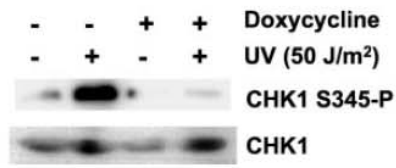

FIG. 4. The role of ATR in CHK2 activation by irofulven. GM00847 human fibroblast expressing tetracycline-controlled, FLAGtagged kinase-dead ATR (ATR.kd) was induced with $1.5 \mu \mathrm{g} / \mathrm{ml}$ of doxytagged kinase-dead ATR (ATR.kd) was induced with $1.5 \mu \mathrm{g} / \mathrm{ml}$ of doxy-
cycline for $48 \mathrm{~h}$. The induced and un-induced cells were then treated cycline for $48 \mathrm{~h}$. The induced and un-induced cells were then treated
with $8 \mu \mathrm{M}$ of irofulven for $1 \mathrm{~h}$ followed by $12 \mathrm{~h}$ of post-treatment incubation. A, ATR.kd induction was shown on the top panel by Western blot with anti-FLAG antibody. The nonspecific band (n.s.) was shown as the loading control for FLAG-ATR.kd induction. CHK2 activation was shown by Western blot with anti-phospho-CHK2 antibody on the bottom panel. The blot for $\mathrm{CHK} 2$ was shown as the loading on the bottom panel. The blot for CHK2 was shown as the loading contri. B,ind light CHK1 activation was determined by Western -CHK1 antibody; the blot for CHK1 was shown as the loading control.

A.

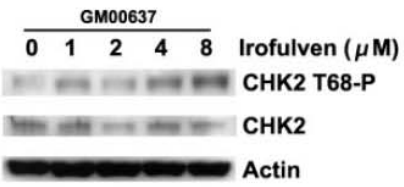

B.

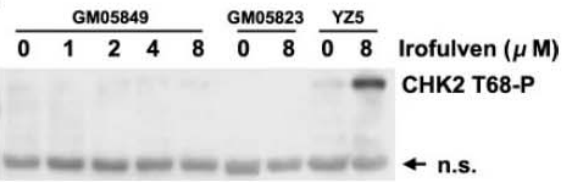

FIG. 5. The role of ATM in CHK2 activation by irofulven. A, SV40-transformed human normal fibroblast GM00637 was treated with different concentrations of irofulven as indicated for $1 \mathrm{~h}$ followed by $24 \mathrm{~h}$ of drug-free incubation. CHK2 activation was determined by Western blot with anti-phospho-CHK2 antibody. Western blots for CHK2 and actin were shown as the loading control. B, SV40-transformed human AT (ataria AT (ataxia telangiectasia fibroblast, GMO5849, its parental non-transATM-complemented AT fibroeated with irofulven. The CHK2 activation was determined by Western blot with anti-phospho-CHK2 antibody, the nonspecific band (n.s.) was shown as the loading control.

drug-free incubation. Western blot assay was carried out with an antibody specifically recognizing the phosphorylated ATM on Ser ${ }^{1981}$. As shown in Fig. 6A, irofulven treatment resulted in ATM kinase phosphorylation on Ser $^{1981}$ in both A2780 and CAOV3 cell lines.

To further confirm the role that ATM played in CHK2 activation in ovarian cancer cells following irofulven treatment, The RNA interference experiment was performed in A2780 cells. As shown in Fig. 6B, ATM protein level was greatly reduced by transfection of siRNA against ATM (siATM), but not by transfection of siRNA against bacterial green fluorescence protein (siGFP). Accordingly, CHK2 activation following
A.

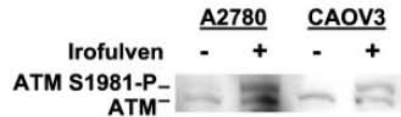

B.

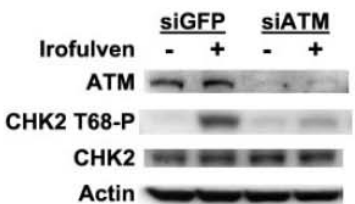

FIG. 6. ATM is phosphorylated following irofulven treatment in ovarian eancer cells and inhibition of ATM expression blocks irofulven-induced CHK2 activation. $A$, ovarian cancer cell lines $\mathrm{A} 2780$ and CAOV3 were treated with their respective $1 \times \mathrm{IC}_{50}$ concentration of irofulven for $1 \mathrm{~h}$ followed by $24 \mathrm{~h}$ of drug-free incubation. The phosphorylation of ATM kinase on Ser $^{1981}$ was determined by Western blot with anti-phospho-Ser ${ }^{1981}$-ATM antibody, B, A2780 cells were transfected with small interfering RNAs for GFP (siGFP) and ATM (siATM) as described under "Experimental Procedures." ATM protein level and CHK2 activation were determined by Western blot with anti-ATM and anti-phospho-CHK2 antibodies. Western blots for CHK2 and actin were used as the loading control.

irofulven treatment was greatly attenuated in cells transfected with siATM (Fig. 6B)

ATM and CHK2 Target Proteins Activated by Irofulven-In response to ionizing radiation, ATM phosphorylates NBS1 on $\operatorname{Ser}^{343}(30-32)$, SMC1 on $\operatorname{Ser}^{957}$ and $\operatorname{Ser}^{966}(33,34)$, p53 on $\operatorname{Ser}^{15}(52,53,87,88)$. ATM and CHK2 phosphorylate BRCA1 on multiple serine sites (36-38). CHK2 phosphorylates p53 on $\operatorname{Ser}^{20}$ and stabilizes p53 $(54,56,59,89)$.

In an effort to further understand irofulven-induced activation of ATM and CHK2, the activation of ATM and CHK2 target proteins was tested. Ovarian cancer cell lines A2780, CAOV3, SKOV3, and OVCAR3 were treated with irofulven, Western blot results demonstrated that NBS1 was phosphorylated on $\mathrm{Ser}^{343}$ in SKOV3 and OVCAR3 cells, but not in A2780 and CAOV3 cells as determined with antibody recognizing phosphorylated NBS1. When the NBS1 protein expression levels were compared, it was shown that much less NBS1 was expressed in A2780 and CAOV3 cells than in SKOV3 and OVCAR3 cells (Fig. 7A). Further, Western blot results also demonstrated that SMC1 was phosphorylated in CAOV3, SKOV3 and OVCAR3 cells as determined by antibody recognizing phosphorylated SMC1 on Ser ${ }^{957}$. Phosphorylation of p53 on $\mathrm{Ser}^{15}$ was observed in A2780 cells harboring wild-type p53 (90) (Fig. 7A). In the three other cell lines, p53 is known to be mutated (91).

To determine the dependence of NBS1, SMC1, and p53 activation on ATM after irofulven treatment, normal human fibroblast GM00637 and AT fibroblast GM05849 were treated with irofulven and Western blot results indicated that more NBS1 was phosphorylated on $\mathrm{Ser}^{343}$ and band-shifted in ATM wildtype GM00637 cells than in AT cells, GM05849. Similarly, more SMC1 phosphorylation on Ser ${ }^{957}$ and p53 phosphorylation on Ser $^{15}$ were observed in ATM wild-type cells than in AT cells (Fig. 7B).

In summary, NBS1, SMC1, and p53 were activated by irofulven both in ovarian cancer cell lines and in normal human fibroblasts, and this activation is dependent on ATM.

CHK2 phosphorylates p53 on Ser $^{20}$ and this phosphorylation event stabilizes the p53 protein $(54-56,59,68)$. However, recent studies suggest that it is unlikely that $\mathrm{CHK} 2$ regulates p53 $(78,79)$. To determine whether irofulven-induced CHK2 
A.

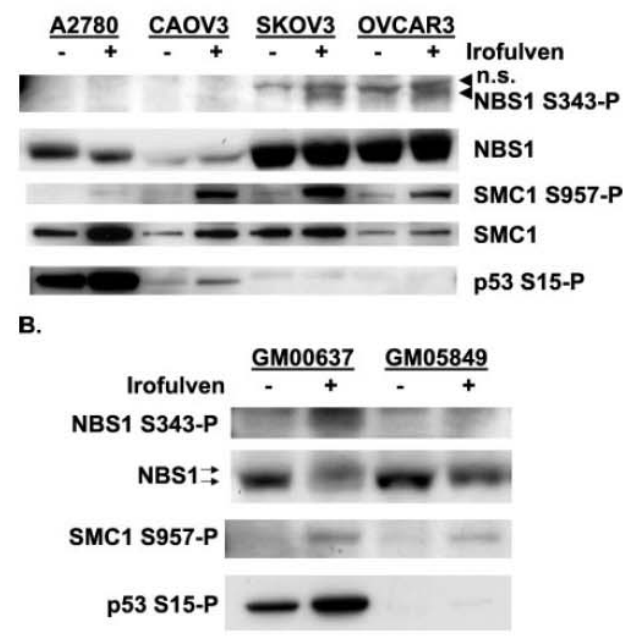

c.

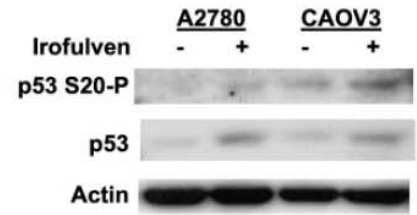

D.

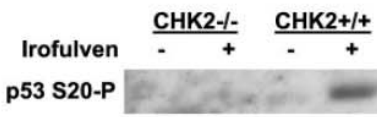

p53

Actin

FIG. 7. ATM and CHK2 target proteins activated by irofulven. $A$ and $B$, human ovarian cancer cell lines A2780, CAOV3, SKOV3, and OVCAR3 were treated with $1 \times \mathrm{IC}_{50}$ concentration of irofulven $(A)$; and human normal fibroblast GM00637 50 AT fibolirofuld human normal fibroblast GM00637 and AT fibroblast GM05849 were treated with $8 \mu \mathrm{M}$ of irofulven $(B)$ for $1 \mathrm{~h}$ followed by additional $24 \mathrm{~h}$ of incubation. Western blot analyses were performed with antibodies against phosphorylated NBS1 on Ser ${ }^{343}$, NBS1, phosphorylated SMC1 on Ser ${ }^{957}$, SMC1, and phosphorylated p53 on $\operatorname{Ser}^{15}$. The nonspecific band was indicated as n.s. $C$ and $D, A 2780$ and CAOV3 cells $(C)$; and human colon cancer cell line HCT116 and its CHK2 knockout subline $(D)$ were treated with $1 \times \mathrm{IC}_{50}$ concentration of irofulven for $1 \mathrm{~h}$ followed by additional $24 \mathrm{~h}$ of incubation, Western blots were performed with antibodies against phosphorylated p53 on $\mathrm{Ser}^{20}, \mathrm{p} 53$, and actin.

activation has any effect on p53 phosphorylation on $\mathrm{Ser}^{20}$, antibody recognizing phosphorylated p53 on $\operatorname{Ser}^{20}$ was used in Western blot analysis. In A2780 (wild-type p53) and CAOV3 (mutated p53) cells after irofulven treatment, p53 phosphorylation and protein accumulation were observed (Fig. 7C). To assess whether p53 phosphorylation on Ser $^{20}$ is dependent on CHK2, the human colon cancer cell line HCT116 and its CHK2 knockout derivative (78) were treated with irofulven and Western blot results indicated that increased phosphorylation of p53 on $\mathrm{Ser}^{20}$, and greater p53 protein accumulation were observed in parental HCT116 cells compared with CHK2 knockout cells, suggesting this phosphorylation event is dependent on CHK2 status (Fig. $7 D$ )

BRCA1 phosphorylation was also determined by Western blot in HCT116 cells and its CHK2 knockout cells treated with irofulven, but no difference on BRCA1 phosphorylation was observed (data not shown).

Taken together, in response to irofulven-induced DNA damage, p53 was phosphorylated on serine 20 in a CHK2-dependent manner.

CHK2 Activation by Irofulven Contributes to $S$ Phase Arrest-Many studies have linked CHK2 activation to $\mathrm{G}_{1}, \mathrm{~S}$ or $\mathrm{G}_{2} / \mathrm{M}$ phase arrest, respectively $(42,54-56,65-67,73,74)$. To understand the possible role that irofulven-induced CHK2 activation might play in cell cycle arrest, we examined the isogenic human colon cancer cell line HCT116 and its CHK2 knockout derivative (78) after irofulven treatment. These paired cell lines were treated with $1 \times \mathrm{IC}_{50}$ concentration of irofulven for $1 \mathrm{~h}$ followed by additional 12 or $24 \mathrm{~h}$ of incubation in drug-free media. Western blot analysis indicated that CHK2 was only expressed and activated in parental HCT116 $(\mathrm{CHK} 2+/+)$ cells (Fig. 8A). To determine the effect of CHK2 activation on cell cycle arrest, the CHK2+l+ and CHK2-Icells were pulse-labeled with BrdU before drug treatment, harvested at different time points and stained with FITC-conjugated anti-BrdU antibody and propidium iodide. FACS analysis for BrdU-positive cells indicated that at time zero, in the untreated control groups, there were $84 \%$ of CHK $2+/+$ cells in $\mathrm{S}$ phase compared with $75 \%$ of $\mathrm{CHK} 2-1-$ cells in $\mathrm{S}$ phase. Three hours after the drug treatment, there were 4-fold more CHK $2+1+$ cells arrested at S phase than CHK $-1-$ cells. This trend was maintained throughout the 24 -h period after drug removal (Fig. $8 B$ ). Similarly, when comparing the $\mathrm{S}$ phase ratio of irofulven-treated over untreated in each cell line, it was clearly shown that there were more cells arrested at $\mathrm{S}$ phase in CHK $2+1+$ cells than in CHK $-1-$ cells (Fig. $8 C$ ).

In response to IR-induced DNA damage, ATM and CHK2 phosphorylate CDC25A on serine 123 , leading to its ubiquitination and degradation, and consequently resulting in S phase arrest $(65,66)$. To further confirm the results obtained with CHK2 knockout cell lines, the ovarian cancer cell line CAOV3 was first treated with irofulven and CDC25A protein level was determined by Western blot. $12 \mathrm{~h}$ after irofulven treatment, CDC25A was degraded. Addition of the proteasome inhibitor, LLnL, could block this degradation, indicating cells were arrested at $\mathrm{S}$ phase at this time point (Fig. 9A). To further study the role that CHK2 activation plays in irofulven-induced S phase arrest, CAOV3 cells were transfected with vector or HA-tagged kinase-dead CHK2 (CHK2.kd) (82). Cells were then treated with irofulven and stained with FITC-conjugated anti-HA antibody and propidium iodide. Results of FACS analysis performed $12 \mathrm{~h}$ post-drug treatment were consistent with CDC25A degradation studies and indicated that in vectortransfected cells, $\sim 38 \%$ of untreated cells were in S phase, whereas $\sim 85 \%$ of irofulven-treated cells were in S phase (Fig. $9, B$ and $C$ ). Among the CHK2.kd-transfected cells, the total cell population presented a similar cell cycle distribution pattern as vector-transfected cells after irofulven treatment. But when cells were gated for only FITC-positive (CHK2.kd-transfected) cells, after irofulven treatment, the number of cells in $\mathrm{S}$ phase was decreased by $20 \%$ (Fig. $9, B$ and $C$ ), indicating that introduction of kinase-dead CHK2 inhibited irofulven-induced $\mathrm{S}$ phase arrest.

Taken together, it was concluded that CHK2 activation by irofulven contributes to $\mathrm{S}$ phase arrest. 
A.

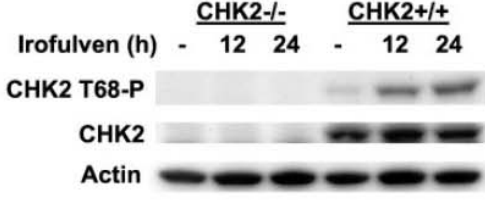

B.

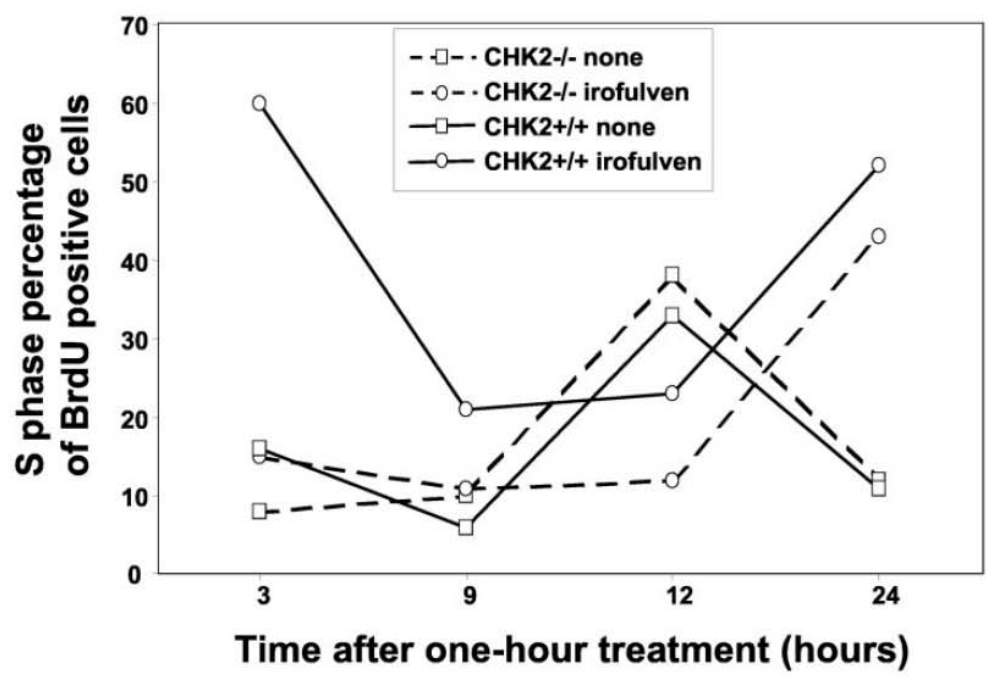

C.

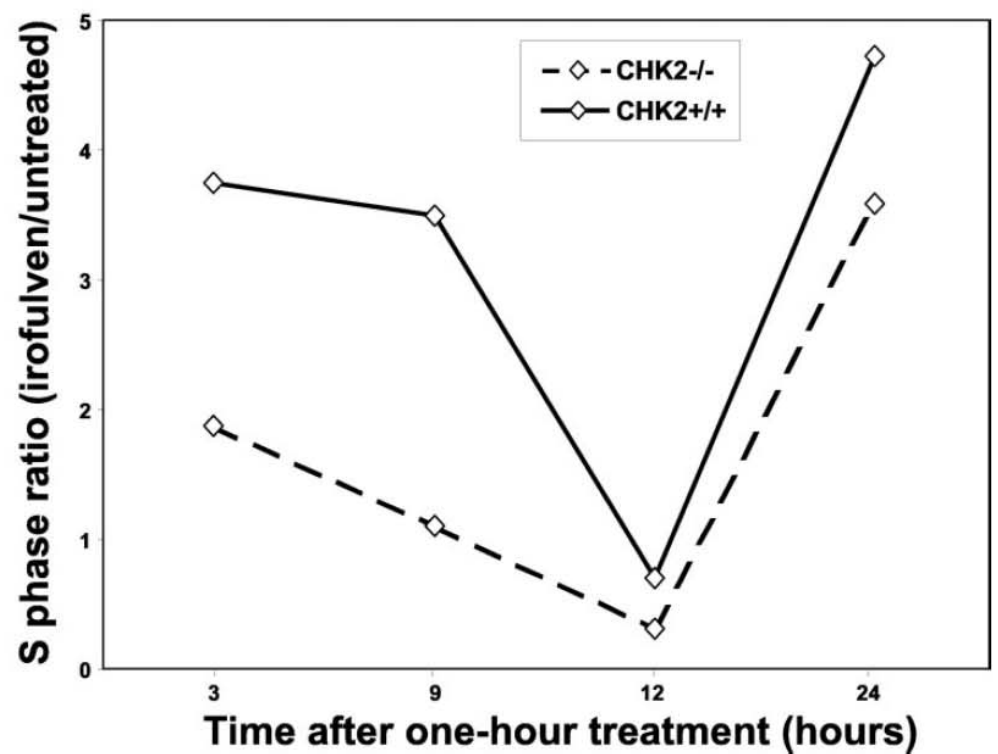

FIG. 8. Irofulven-induced CHK2 activation contributes to $\mathrm{S}$ phase arrest. $A$, human colon cancer cell line HCT116 and its CHK2 knockout derivative were treated with $1 \times \mathrm{IC}_{50}$ concentration of irofulven for $1 \mathrm{~h}$ followed by additional 12 or $24 \mathrm{~h}$ of incubation, CHK2 activation was observed by Western blot with antibody recognizing the phosphorylated form of CHK2 kinase on Thr ${ }^{68}$. Western blots for CHK2 and actin served as loading control. $B$ and $C$, HCT116 CHK2+/+ and CHK2-/ - cells were pulse-labeled with $10 \mu \mathrm{M}$ of BrdU for $1 \mathrm{~h}$ followed by 1 -h drug treatment at $1 \times \mathrm{IC}_{50}$ concentration. Untreated and treated cells were harvested at different time point and stained with FITC-conjugated anti-BrdU antibody and propidium iodide. Cells were then applied to FACS analysis. The BrdU-positive cells were gated and histograms of DNA content versus cell counts (30,000 events) were obtained. The percentage of each cell cycle phase was analyzed by ModFit v3.0 software. $\mathrm{S}$ phase percentage of ( irofulven-treated over untreated for $\mathrm{CHK} 2+/+$ and $\mathrm{CHK} 2-/-$ cells was shown in $C$. 
A.

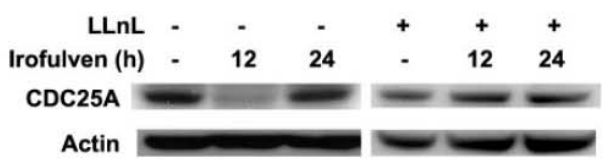

B.

FIG. 9. Irofulven induces CDC25A degradation and expression of $\mathrm{ki}$ degradation nase-dead CHK2 inhibits irofulveninduced $\mathrm{S}$ phase arrest. $A$, ovarian cancer cell line CAOV3 was treated with $1 \times$ $\mathrm{IC}_{50}$ concentration of irofulven for $1 \mathrm{~h}$ followed by additional $24 \mathrm{~h}$ incubation; Western blots were performed with antibodies against CDC25A and actin. To inhibit CDC25A degradation, cells were cultured in the presence of the proteasome ritor 150 . nhibitor LLnL ( $50 \mu \mathrm{M})$. LLnL was added CA min before AOV cells were transfected with vector or HA-tagged kinase-dead CHK2 (CHK2.kd) as described under "Experimental Procedures." Cells were stained with FITC-conjugated anti-HA antibody and propidium iodide. Cells were then applied to FACS analysis. Triplicate experiments were carried out. The FITC-positive cells were gated and histograms of DNA content versus cell counts were obDNA content versus cell counts we obtained. In the vector-transfected group,

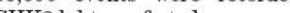
CHK2.kd-transfected group, ten times more events were recorded to overcome the low transfection efficiency. $C, \mathrm{~S}$ phase percentage of vector and CHK2.kd-transfected cells with or without irofulven treatment.
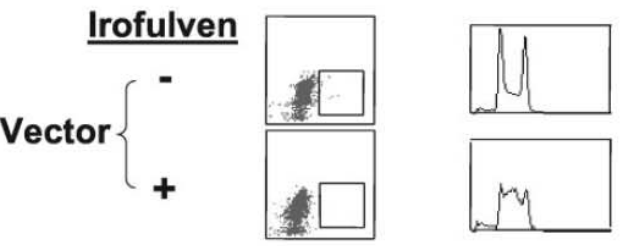

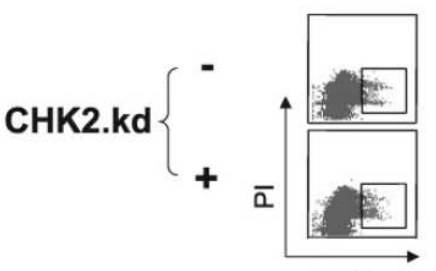

FITC

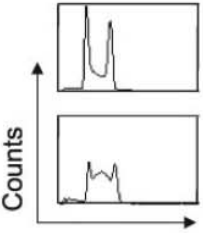

$\mathrm{PI}$

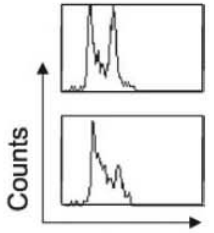

FITC (+)-PI

C.

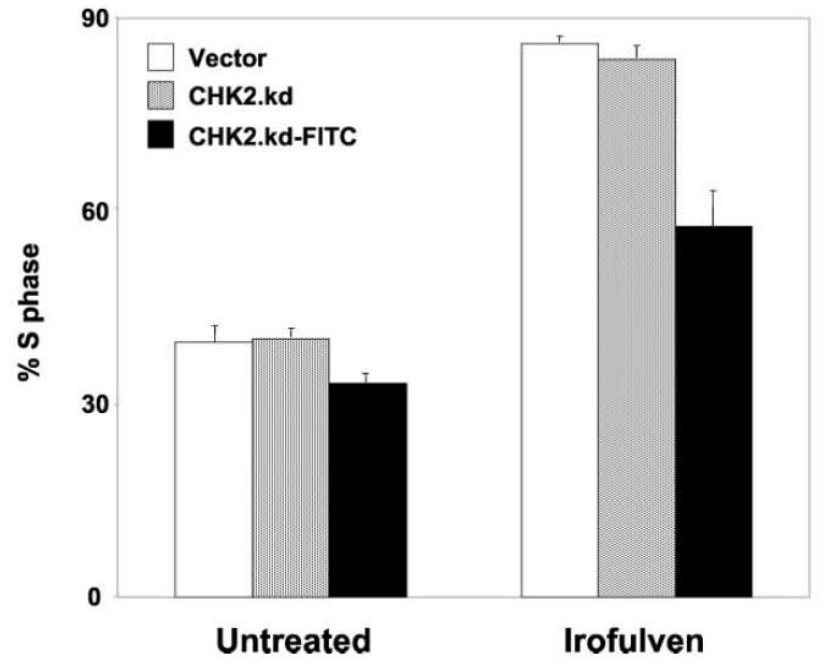

DISCUSSION

Irofulven is a novel semi-synthetic derivative of the mushroom toxin, illudin $\mathrm{S}$, which has demonstrated antitumor activity against prostate, pancreatic, and ovarian cancer in clinical trials. The mechanism of irofulven action involves several biological processes including DNA damage, MAPK signaling, cell cycle arrest, and caspase-dependent apoptosis. The chemical structure and nature of irofulven-elicited DNA lesions are currently unknown. By using paired cell lines proficient and deficient for ATM, ATR or CHK2 function, and by using RNA interference techniques and transfection of dominant-negative
CHK2, we show that irofulven induces ATM-dependent CHK2 activation leading to $\mathrm{S}$ phase arrest, a pathway that has primarily been characterized in response to ionizing radiationinduced DNA double strand breaks (23-29). In contrast, the ATR and CHK1 pathway, which mainly responds to drug and UV-induced DNA lesions (23-29), does not play an important role in irofulven-induced DNA damage response. In addition, we also show that ATM target proteins NBS1, SMC1, and p53 were also phosphorylated in an ATM-dependent manner upon irofulven treatment, and p53 phosphorylation on serine 20 induced by irofulven is dependent on CHK2 status. These novel 
observations may aid in the further elucidation of the molecular mechanisms of action of irofulven, and in the potentia design of combined therapy with radiation, $\mathrm{S}$ phase abrogators, or inhibitors of the ATM-CHK2 signaling pathway.

CHK2 has been shown to be involved in DNA repair process at stalled replication forks by interacting with ATR, p53, and BRCA1 (28, 92-94). A recent study done in fission yeast demonstrated that Cds1 (CHK2) interacts with Rad60, a protein required for recombinational repair in fission yeast. Cds1 activation triggers Rad60 phosphorylation and nuclear delocalization regulating recombination events at stalled replication forks (95). While it is yet to be determined whether CHK2 activation might also play a role in repairing irofulven-elicited DNA lesions, it has been reported that irofulven-induced DNA damage is repaired by transcription-coupled nucleotide excision repair (TC-NER) (21). Therefore, it can be speculated that the CHK2 activation by irofulven might be the result of stalled replication machinery. Supporting this idea, CHK2 activation by irofulven in ovarian cancer cell lines has been found to be replication and transcription-dependent (data not shown). Interestingly, it has also been reported that DNA doublestranded breaks (DSB) are observed in irofulven-treated cells (96). Therefore, having a better understanding of the types of lesions formed by irofulven will help in uncovering the novel recognition and signaling pathway evoked by this drug.

In response to IR-induced DNA damage, ATM or ATR directly phosphorylates p53, NBS1, SMC1, MDM2, and BRCA1 (23,30-37,50-53). Activation of NBS1, SMC1, and BRCA1 plays a very important role in $\mathrm{S}$ and $\mathrm{G}_{2} / \mathrm{M}$ checkpoint control $(22,23,30-41,50-53,66,97,98)$. Activation of SMC1 has also been shown to contribute to radiosensitivity (33). Recent studies demonstrated that parallel pathways exist in radiationinduced intra-S phase checkpoint. ATM phosphorylates CHK2 and NBS1, two branches of the ATM-mediated DNA damage response pathway (ATM-CHK2-CDC25A-CDK2 and ATMNBS1-MRE11-RAD50), each of which partially controls intra-S phase arrest $(30,32,34,65,66,99)$. We demonstrated in this study that irofulven induces phosphorylation of NBS1 on $\mathrm{Ser}^{343}$, SMC1 on Ser ${ }^{957}$, and p53 on $\mathrm{Ser}^{15}$ in an ATM-dependent manner, and induces p53 phosphorylation on $\mathrm{Ser}^{20}$-dependent on CHK2 status. The potential roles of NBS1, SMC1, and BRCA1 in irofulven-induced DNA damage response, CHK2 activation, and chemosensitivity provide avenues for future research.

It has been previously determined that CHK2 regulates $\mathrm{G}_{1}$ arrest by activating p53 (54-56), S phase checkpoint by phosphorylating CDC25A (65-67), or $\mathrm{G}_{2} / \mathrm{M}$ transition by phosphorylating CDC25C $(42,73,74)$ following DNA damage. However, controversy exists regarding the role of $\mathrm{CHK} 2$ in cell cycle regulation. One report suggested that $\mathrm{CHK} 2$ is dispensable for p53-mediated $\mathrm{G}_{1}$ arrest (77), while other studies demonstrated that cells from CHK2 knockout mice have normal S phase and $\mathrm{G}_{2} / \mathrm{M}$ transition $(56,57)$. Additionally, it has been argued that it is unlikely CHK1 and CHK2 are regulators of p53 or the $G_{1}$ and $\mathrm{G}_{2}$ checkpoints activated by $\mathrm{IR}(78,79)$. Yet, CHK2 has been shown to play a partial role in controlling the $\mathrm{S}$ phase checkpoint upon IR treatment (66). In this study, we found that CHK2 contributes to the $\mathrm{S}$ phase arrest induced by irofulven. By BrdU labeling and analysis of BrdU-positive cells, S phase delay between 9 - and 12 -h period in irofulven-treated, BrdUpositive CHK2-/- and CHK2+/+ cells was observed. An increase of S phase cells was also observed in CHK2-/- cells treated with irofulven, but the number of cells arrested in $\mathrm{S}$ phase in $\mathrm{CHK} 2+/+$ cells was constantly higher than in CHK2-/- cells. The magnitude of differences in $\mathrm{S}$ phase arrest between irofulven-treated CHK2 $+/+$ and CHK2-/- cells de- clined over the time. Because cells were treated with relatively low $\left(1 \times \mathrm{IC}_{50}\right)$ drug concentrations, it is possible that this is related to the ability of viable cells to recover from drug insult. In addition, by transfecting kinase-dead CHK2 into ovarian cancer cells, FACS analysis results indicated that $\mathrm{S}$ phase arrest induced by irofulven was inhibited.

CHK2 has also been implicated in apoptosis induction (56, $77,85,100-102)$. We have previously found that irofulven strongly induces JNK and ERK activation and caspase-mediated apoptosis in pancreatic cancer cell lines $(19,20)$. There have been reports indicating that the JNK or ERK activation in response to DNA damage might be regulated by ATM- or ATRinitiated pathways (103-107). Therefore, the possible role of CHK2 activation in irofulven-induced cell death and the link between DNA-damage response and MAPK activation induced by irofulven remain to be elucidated.

In summary, irofulven, a novel anticancer agent, activates a DNA damage signaling pathway by triggering ATM-dependent activation of NBS1, SMC1, CHK2 and p53 activation. The degree of CHK2 activation is dependent on both irofulven concentration and length of exposure. Furthermore, it was demonstrated that CHK2 activation contributes to irofulven-induced S phase arrest.

Acknowledgments - We thank the following people for generously providing reagents: Bert Vogelstein and Fred Bunz (Johns Hopkins University, Baltimore, MD) for human colon cancer cell line HCT116 and its isogenic CHK2 knockout derivative HCT116 CHK2-/-; and its isogenic CHK2 knockout derivative HCT116 CHK2-l-; Stuart L. Schreiber and Shlomo Handeli (Fred Hutchinson Cancer
Research Center, Seattle, WA) for the GM00847 human fibroblast exResearch Center, Seattle, WA) for the GM00847 human fibroblast ex-
pressing kinase-dead ATR; Yosef Shiloh (Tel Aviv University, Israel) for pressing kinase-dead ATR; Yosef Shiloh (Tel Aviv University, Israel) for
the ATM-complemented AT fibroblast (AT22IJE-T-pEBS7-YZ5); and Jann Sarkaria (Mayo Clinic and Foundation, Rochester, MN) for kinase-dead CHK2. We also would like to thank Drs. Dan Flynn and Xianglin Shi for critically reading the manuscript.

\section{REFERENCES}

1. Kelner, M. J., McMorris, T. C., Rojas, R. J., Trani, N. A., and Estes, L. (2002) Cancer Chemother. Pharmacol. 49, 412-418

MacDonald, J. R., Muscoplat, C. C., Dexter, D. L., Mangold, G. L., Chen, S. F., Kelner, $\mathrm{M}$

3. Kelner, M. J., McMorris, T. C., Estes, L., Samson, K. M., Bagnell, R. D., and Taetle, R. (1998) Eur. J. Cancer 34, 908-913

4. Kelner, M. J., McMorris, T. C., Estes, L., Wang, W., Samson, K. M., and Taetle, R. (1996) Investig. New Drugs 14, 161-167

5. Kelner, M. J., McMorris, T. C., Estes, L. A., Oval, M. Y., Rojas, R. J., Lynn, J. R., Lanham, K. A., and Samson, K. M. (2000) Anticancer Drugs 11, $217-224$

6. Sato, Y., Kashimoto, S., MacDonald, J. R., and Nakano, K. (2001) Eur. J. Cancer $37,1419-1428$

7. Friedman, H. S., Keir, S. T., Houghton, P. J., Lawless, A. A., Bigner, D. D., and Waters, S. J. (2001) Cancer Chemother. Pharmacol. 48, 413-416

8. Kelner, M. J., McMorris, T. C., and Taetle, R. (1990) J. Natl. Cancer Inst. 82, $1562-1565$

9. Hammond, L. A., Hilsenbeck, S. G., Eckhardt, S. G., Marty, J., Mangold, G., MacDonald, J. R., Rowinsky, E. K., Von Hoff, D. D., and Weitman, S. (2000) Eur. J. Cancer 36, 2430-2436

Hidalgo, M., Izbicka, E., Eckhardt, S. G., MacDonald, J. R., Cerna, C., Gomez,
L., Rowinsky, E. K., Weitman, S. D., and Von Hoff L., Rowinsky, E. K.,

11. Britten, C. D., Hilsenbeck, S. G., Eckhardt, S. G., Marty, J., Mangold, G., MacDonald, J. R., Rowinsky, E. K., Von Hoff, D. D., and Weitman, S. (1999) Cancer Res. 59, 1049-1053

12. Seiden, M. V. (2001) Oncologist 6, 327-332

13. Giles, F., Cortes, J., Garcia-Manero, G., Kornblau, S., Estey, E., Kwari, M., Murgo, A., and Kantarjian, H. (2001) Investig. New Drugs 19, 13-20

4. Eckhardt, S. G., Baker, S. D., Britten, C. D., Hidalgo, M., Siu, L., Hammond, L. A., Villalona-Calero, M. A., Felton, S., Drengler, R., Kuhn, J. G., Clark, G. M., Smith, S. L., MacDonald, J. R., Smith, C., Moczygemba, J., Weitman, S., Von Hoff, D. D., and Rowinsky, E. K. (2000) J. Clin. Oncol. 18, $4086-4097$

15. Murgo, A., Cannon, D. J., Blatner, G., and Cheson, B. D. (1999) Oncology (Huntingt.) 13, 237-238

16. Kelner, M. J., McMorris, T. C., Rojas, R. J., Trani, N. A., Velasco, T. R., Estes, L. A., and Suthipinijtham, P. (2002) Investig. New Drugs 20, 271-279 7. Poindessous, V., Koeppel, F., Raymond, E., Comisso, M., Waters, S. J., and Larsen, A. K. (2003) Clin. Cancer Res. 9, 2817-2825

Kelner, M. J., McMorris, T. C., Beck, W. T., Zamora, J. M., and Taetle, R. (1987) W Wes. 47, 3186-3189

Miller, A. R. (2001) Anticancer Res. R., Von Hoff, D. D., Strodel, W. E., and 

20. Wang, W., Waters, S. J., MacDonald, J. R., Roth, C., Shentu, S., Freeman, J.
Von Hoff, D. D., and Miller, A. R. (2002) Anticancer Res. 22, 559-564 21. Jaspers, N. G., Raams, A., Kelner, M. J., Ng, J. M., Yamashita, Y. M., Taked, S., McMorris, T. C., and Hoeijmakers, J. H. (2002) DNA Repair (Amst.) 1, $1027-1038$

22. Sherr, C. J. (2000) Cancer Res. 60, 3689-3695

23. Zhou, B. B., and Elledge, S. J. (2000) Nature 408, 433-439

Barlow, C., Hirotsune, S., Paylor, R., Liyanage, M., Eckhaus, M., Collins, F.,
Shiloh, Y., Crawley, J. N., Ried, T., Tagle, D., and Wynshaw-Boris, A. (1996) Cell 86, 159-171

25. Xu, Y., Ashley, T., Brainerd, E. E., Bronson, R. T., Meyn, M. S., and Baltimore, A

26. Brown, E. J., and Baltimore, D. (2000) Genes Dev. 14, 397-402

27. de Klein, A., Muijtjens, M., van Os, R., Verhoeven, Y., Smit, B., Carr, A. M., Lehmann, A. R., and Hoeijmakers, J. H. (2000) Curr. Biol. 10, 479-482

28. Abraham, R. T. (2001) Genes Dev. 15, 2177-2196

29. Liu, Q., Guntuku, S., Cui, X. S., Matsuoka, S., Cortez, D., Tamai, K., Luo, G., Carattini-Rivera, S., DeMayo, F., Bradley, A., Donehower, L. A., and Elledge, S. J. (2000) Genes Dev. 14, 1448-1459

30. Lim, D. S., Kim, S. T., Xu, B., Maser, R. S., Lin, J., Petrini, J. H., and Kastan, M. B. (2000) Nature 404, 613-617

31. Gatei, M., Young, D., Cerosaletti, K. M., Desai-Mehta, A., Spring, K., Kozlov, S., Lavin, M. F., Gatti, R. A., Concannon, P., and Khanna, K. (2000) Nat. Genet. 25, 115-119

32. Wu, X., Ranganathan, V., Weisman, D. S., Heine, W. F., Ciccone, D. N., O'Neill, T. B., Crick, K. E., Pierce, K. A., Lane, W. S., Rathbun, G., Livingston, D. M., and Weaver, D. T. (2000) Nature 405, 477-482

33. Kim, S. T., Xu, B., and Kastan, M. B. (2002) Genes Dev. 16, 560-570
34. Yazdi, P. T., Wang, Y., Zhao, S., Patel, N., Lee, E. Y., and Qin, J. (2002) Genes Dev. 16, $571-58$

35. Maya, R., Balass, M., Kim, S. T., Shkedy, D., Leal, J. F., Shifman, O., Moas, M., Buschmann, T., Ronai, Z., Shiloh, Y., Kastan, M. B., Katzir, E., and Oren, M. (2001) Genes Dev. 15, 1067-107

36. Li, S., Ting, N. S., Zheng, L., Chen, P. L., Ziv, Y., Shiloh, Y., Lee, E. Y., and Lee, W. H. (2000) Nature $406,210-21$

37. Gatei, M., Scott, S. P., Filippovitch, I., Soronika, N., Lavin, M. F., Weber, B., Khanna, K. K. (2000) Cancer Res. 60, 3299

38. Lee, J. S., Collins, K. M., Brown, A. L., Lee, C. H., and Chung, J. H. (2000) Nature 404, 201-204

39. Xu, B., O'Donnell, A. H., Kim, S. T., and Kastan, M. B. (2002) Cancer Res. 62 , $4588-4591$

40. Xu, B., Kim, S. T., Lim, D. S., and Kastan, M. B. (2002) Mol. Cell. Biol. 22, $1049-1059$

41. Xu, B., Kim, S., and Kastan, M. B. (2001) Mol. Cell. Biol. 21, 3445-3450

42. Matsuoka, S., Huang, M., and Elledge, S. J. (1998) Science 282, 1893-1897

43. Matsuoka, S., Rotman, G., Ogawa, A., Shiloh, Y., Tamai, K., and Elledge, S. J. (2000) Proc. Natl. Acad. Sci. U. S. A. 97, 10389-10394 and Elledge, S. J.

44. Melchionna, R., Chen, X. B., Blasina, A., and McGowan, C. H. (2000) Nat. Cell Biol. 2, 762-765

45. Brown, A. L., Lee, C. H., Schwarz, J. K., Mitiku, N., Piwnica-Worms,
Chung, J. H. (1999) Proc. Natl. Acad. Sci. U. S. A. 96, 3745-3750

46. Chaturvedi, P., Eng, W. K., Zhu, Y., Mattern, M. R., Mishra, R., Hurle, M. R., Chang, X., Annan, R. S., Lu, Q., Faucette, L. F., Scott, G. F., Li, X., Carr, S. A., Johnson, R. K., Winkler, J. D., and Zhou, B. B. (1999) Oncogene 18, 4047-4054

47. Zhao, H., and Piwnica-Worms, H. (2001) Mol. Cell. Biol. 21, 4129-4139

48. Sanchez, Y., Wong, C., Thoma, R. S., Richman, R., Wu, Z., Piwnica-Worms, H., and Elledge, S. J. (1997) Science 277, 1497-1501

49. Gatei, M., Sloper, K., Sorensen, C., Syljuasen, R., Falck, J., Hobson, K. Savage, K., Lukas, J., Zhou, B. B., Bartek, J., and Khanna, K. K. (2003) J. Biol. Chem. 278, 14806-14811

50. Lakin, N. D., Hann, B. C., and Jackson, S. P. (1999) Oncogene 18, 3989-3995 51. Kim, S. T., Lim, D. S., Canman, C. E., and Kastan, M. B. (1999) J. Biol. Chem.

52. Can, C.E.

Canman, C. E., Lim, D. S., Cimprich, K. A., Taya, Y., Tamai, K., Sakaguchi, K. Appella,

53. Dumaz, N., and Meek, D. W. (1999) EMBO J. 18, 7002-7010

54. Chehab, N. H., Malikzay, A., Appel, M., and Halazonetis, T. D. (2000) Genes Dev, $14,278-288$

55. Shieh, S. Y., Ahn, J., Tamai, K., Taya, Y., and Prives, C. (2000) Genes Dev. 14, $289-300$

56. Hirao, A., Kong, Y. Y., Matsuoka, S., Wakeham, A., Ruland, J., Yoshida, H., Liu, D., Elledge, S. J., and Mak, T. W. (2000) Science 287, 1824-1827

57. Takai, H., Naka, K., Okada, Y., Watanabe, M., Harada, N., Saito, S., Anderson, C. W., Appella, E., Nakanishi, M., Suzuki, H., Nagashima, K., Sawa, H.,
Ikeda, K., and Motoyama, N. (2002) EMBO J. 21, 5195-5205 58. Craig, A., Scott, M., Burch, L., Smith, G., Ball, K., and Hupp, T. (2003) EMBO Rep 4, 787-792

59. Chehab, N. H., Malikzay, A., Stavridi, E. S., and Halazonetis, T. D. (1999) Proc. Natl. Acad. Sci. U. S. A. 96, 13777-13782

60. Bunz, F., Dutriaux, A., Lengauer, C., Waldman, T., Zhou, S., Brown, J. P., Sedivy, J. M., Kinzler, K. W., and Vogelstein, B. (1998) Science 282, $1497-1501$

61. Zachos, G., Rainey, M. D., and Gillespie, D. A. (2003) EMBO J. 22, 713-723
62. Levine, A. J. (1997) Cell $88,323-331$

63. Prives, C. (1998) Cell $95,5-8$

64. Prives, C., and Hall, P. A. (1999) J Pathol 187, 112-126

65. Falck, J., Mailand, N., Syljuasen, R. G., Bartek, J., and Lukas, J. (2001) Nature $410,842-847$

66. Falck, J., Petrini, J. H., Williams, B. R., Lukas, J., and Bartek, J. (2002) Nat. Genet. 30, 290-294

67. Sorensen, C. S., Syljuasen, R. G., Falck, J., Schroeder, T., Ronnstrand, L., Khanna, K. K., Zhou, B. B., Bartek, J., and Lukas, J. (2003) Cancer Cell 3, $247-258$

68. Barter, J, and Lukas, J. (2003) Cancer Cell 3, 421-429

69. Feijoo, C., Hall-Jackson, C., Wu, R., Jenkins, D., Leitch, J., Gilbert, D. M., and Smythe, C. (2001) J. Cell Biol. 154, 913-923

70. Zhou, X. Y., Wang, X., Hu, B., Guan, J., Mliakis, G., and Wang, Y. (2002) Cancer

71. Zhao, H., Watkins, J. L., and Piwnica-Worms, H. (2002) Proc. Natl. Acad. Sci. U.S. A. 99, 14795-14800

72. Zeng, Y., Forbes, K. C., Wu, Z., Moreno, S., Piwnica-Worms, H., and Enoch, T. (1998) Nature 395, 507-510

73. Blasina, A., de Weyer, I. V., Laus, M. C., Luyten, W. H., Parker, A. E, and McGowan, C. H. (1999) Curr. Biol. 9, 1-10

74. Furnari, B., Blasina, A., Boddy, M. N., McGowan, C. H., and Russell, P. (1999) Mol. Biol. Cell 10, 833-845

75. Chen, Z., Xiao, Z., Chen, J., Ng, S. C., Sowin, T., Sham, H., Rosenberg, S., Fesik, S., and Zhang, H. (2003) Mol. Cancer Ther. 2, 543-548

76. Graves, P. M., and Piwnica-Worms, H. (2000) J. Biol. Chem. 275, 5600-5605 77. Jack, M. T., Woo, R. A., Hirao, A., Cheung, A., Mak, T. W., and Lee, P. W. 2002) Proc. Natl. Acad. Sci. U.S. A. 99, 9825-9829

78. Jallepalli, P. V., Lengauer, C., Vogelstein, B., and Bunz, F. (2003) J. Biol. Chem. 278, 20475-20479

79. Ahn, J., Urist, M., and Prives, C. (2003) J. Biol. Chem. 278, 20480-20489 80. Ziv, Y., Bar-Shira, A., Pecker, I., Russell, P., Jorgensen, T. J., Tsarfati, I., and Shiloh, Y. (1997) Oncogene 15, 159-167

81. Cliby, W. A., Roberts, C. J., Cimprich, K. A., Stringer, C. M., Lamb, J. R., Schreiber, S. L., and Friend, S. H. (1998) EMBO J. 17, 159-169

22 Busby, E. C., Leistritz, D. F., Abraham, R. T., Karnitz, L. M., and Sarkaria, J. N. (2000) Cancer Res. 60, 2108-2112

83. Ahn, J. Y., Schwarz, J. K., Piwnica-Worms, H., and Canman, C. E. (2000) Cancer Res. 60, 5934-5936

84. Shiloh, Y. (2001) Curr. Opin. Genet. Dev. 11, 71-77

5. Hirao, A., Cheung, A., Duncan, G., Girard, P. M., Elia, A. J., Wakeham, A., Okada, H., Sarkissian, T., Wong, J. A., Sakai, T., De Stanchina, E., Bristow, R. G., Suda, T., Lowe, S. W., Jeggo, P. A., Elledge, S. J., and Mak, T. W. (2002) Mol. Cell. Biol. 22, 6521-6532

86. Bakkenist, C. J., and Kastan, M. B. (2003) Nature 421, 499-506

87. Banin, S., Moyal, L., Shieh, S., Taya, Y., Anderson, C. W., Chessa, L., Smorodinsky, N. I., Prives, C., Reiss, Y., Shiloh, Y., and Ziv, Y. (1998) Science 281, 1674-1677

88. Siliciano, J. D., Canman, C. E., Taya, Y., Sakaguchi, K., Appella, E., and Kastan, M. B. (1997) Genes Dev. 11, 3471-3481

89. Shieh, S. Y., Taya, Y., and Prives, C. (1999) EMBO J. 18, 1815-1823

90. Brown, R., Clugston, C., Burns, P., Edlin, A., Vasey, P., Vojtesek, B., and Kaye, S. B. (1993) Int. J. Cancer 55, 678-684

91. Yaginuma, Y., and Westphal, H. (1992) Cancer Res. 52, 4196-4199

92. Ward, I. M., and Chen, J. (2001) J. Biol. Chem. 276, 47759-47762

93. Tibbetts, R. S., Brumbaugh, K. M., Williams, J. M., Sarkaria, J. N., Cliby, W. A., Shieh, S. Y., Taya, Y., Prives, C., and Abraham, R. T. (1999) Genes Dev. 13, 152-15

94. Tibbetts, R. S., Cortez, D., Brumbaugh, K. M., Scully, R., Livingston, D., Elledge, S. J., and Abraham, R. T. (2000) Genes Dev. 14, 2989-3002

95. Boddy, M. N., Shanahan, P., McDonald, W. H., Lopez-Girona, A., Noguchi, E. Yates, I. J., and Russell, P. (2003) Mol. Cell. Biol. 23, 5939-5946

arowski, J. M., Napier, C., Koester, S. K., Chen, S. F., Troyer, D. Chapman, W., and MacDonald, J. R. (1997) Biochem. Pharmacol. 54, $1181-1193$

97. Hall-Jackson, C. A., Cross, D. A., Morrice, N., and Smythe, C. (1999) Oncogene 707-6713

98. Cortez, D., Wang, Y., Qin, J., and Elledge, S. J. (1999) Science 286, 1162-1166 Goldberg, M., Stucki, M., Falck, J., D'Amours, D., Rahman, D., Pappin, D., Bartek, J., and Jackson, S. P. (2003) Nature 421, 952-956

100. Xu, J., Xin, S., and Du, W. (2001) FEBS Lett. 508, 394-398 Snow, B., Arya, S., Wong, J., Bouchard, D., Binari, R., Manoukian, A. S., and Mak, T. W. (2002) Proc. Notl. Acad. Sci. U. S. A. 99, 11305-11310 102. Yang, S., Kuo, C., Bisi, J. E., and Kim, M. K. (2002) Nat. Cell Biol. 4, 865-870 103. Zhang, Y., Ma, W. Y., Kaji, A., Bode, A. M., and Dong, Z. (2002) J. Biol. Chem. $277,3124-3131$

104. Pearce, A. K, and Humphrey, T. C. (2001) Trends Cell Biol. 11, 426-433

105. Tang, D., Wu, D., Hirao, A., Lahti, J. M., Liu, L., Mazza, B., Kidd, V. J., Mak, T. W., and Ingram, A. J. (2002) J. Biol. Chem. 277, 12710-12717

106. Shafman, T. D., Saleem, A., Kyriakis, J., Weichselbaum, R., Kharbanda, S., and Kufe, D. W. (1995) Cancer Res. 55, 3242-3245 107. Lee, S. A., Dritschilo, A., and Jung, M. (1998) J. Biol. Chem. 273,
32889-32894 


\title{
Timothy D. Wiltshire, Ph. D.
}

\author{
West Virginia University \\ Mary Babb Randolph Cancer Center \\ Program in Cancer Cell Biology \\ PO Box 9300, Room 1835 \\ Morgantown, WV 26506 \\ 304-293-5246 \\ twiltshire@hsc.wvu.edu
}

\section{Education}

PhD Candidate - West Virginia University, 8/02- 12/07.

Cancer Cell Biology Program

Dr. Weixin Wang-Advisor

Dissertation project - DNA damage response and chemosensitivity to anticancer agent, Irofulven.

Bachelor of Science - Evangel University, Springfield, Missouri, May 1998.

Biology Major, Chemistry Minor

Research and Professional Experience

2002-present PhD Candidate, Department of Microbiology, Immunology and Cell Biology, Cancer Cell Biology Program, West Virginia University, Morgantown, WV.

2003-2005 Teaching Assistant, Department of Microbiology, Immunology, and Cell Biology, West Virginia University, Morgantown, WV.

2000-2002 Production Scientist, Beckman Coulter Incorporated, Chaska, MN.

1996-1999 Production Technician II, Midland Bioproducts Corporation, Boone, IA.

1994-1996 General and Organic Chemistry Lab Assistant, Evangel University, Springfield, MO.

Awards and Professional Memberships

Associate Member - American Association for Cancer Research. 2005-current.

Van Liere Memorial Research Convocation-3rd Place, Oral Presentation, 2006. 
Van Liere Memorial Research Convocation-1st Place, Poster Presentation, 2006.

Graduate Student Representative - elected position. Department of Microbiology, Immunology and Cell Biology, 2004-2005.

Publications

Timothy Wiltshire, Steven Frisch, Eddie Reed and Weixin Wang. Caspase 2 is involved in initiating irofulven -induced cell death. Submitted for Publication.

Timothy Wiltshire, Jamie Senft, Yutian Wang, Gregory W. Konat, Sharon L. Wenger, Eddie Reed, and Weixin Wang. BRCA1 Contributes to Cell Cycle Arrest and Chemoresistance in Response to Anticancer Agent, Irofulven. Molecular Pharmacology. 2007 Apr;71(4):1051-60.

Yutian Wang, Timothy Wiltshire, Jamie Senft, Eddie Reed, and Weixin Wang. Irofulven induces replication-dependent CHK2 activation related to p53 status, Biochemical Pharmacology. 2007 Feb 15:73(4):469-80.

Yutian Wang, Timothy Wiltshire, Jamie Senft, Sharon L. Wenger, Eddie Reed, and Weixin Wang. Fanconi anemia D2 protein confers chemoresistance in response to the anticancer agent, irofulven. Molecular Cancer Therapeutics. 2006 Dec. 5(12):3153-61.

Jian Wang, Timothy Wiltshire, Yutian Wang, Carmenza Mikell, Julian Burks, Cynthia Cunningham, Emily S. Van Laar, Stephen J. Waters, Eddie Reed, and Weixin Wang. ATM-dependent CHK2 Activation Induced by Anticancer Agent, Irofulven. The Journal of Biological Chemistry. 2004 Sep

17;279(38):39584-92.

Abstracts

Yutian Wang, Timothy Wiltshire, Jamie Senft, Sharon L. Wenger, Emily S. Van Laar, Shannon Wadman, Stephen J. Waters, Eddie Reed, and Weixin Wang. Fanconi anemia D2 protein confers chemoresistance and contributes to S phase cell cycle arrest in response to the anticancer agent, irofulven. AACR Meeting Abstracts, Apr 2006; 2006: 123 - 124.

Weixin Wang, Timothy Wiltshire, Yutian Wang, Sharon L. Wenger, Emily S. Van Laar, Stephen J.Waters, and Eddie Reed. Chromosome aberrations, DNA damage signaling and chemosensitivity induced by the anticancer agent, irofulven. AACR Meeting Abstracts, Apr 2005; 2005: 142. 